\title{
Social Science Genetics and Fertility
}

Essays on the Interplay Between Genes, Social Environment and Human Fertility

Felix Christian Tropf 
To friends and family

Design cover:

Frédéric Maurel

www.kompleetsysteem.com

www.fredmaurel.com

(C) 2016 by Felix Christian Tropf

ISBN: 978-94-6299-397-6

All Rights Reserved. No part of this publication may be reproduced in any form or by any means, including scanning, photocopying, or otherwise without prior written permission of the copyright holder.

First Printing, 2016

Printed by: Ridderprint BV, the Netherlands 


\section{university of groningen}

\section{Social Science Genetics and Fertility}

Essays on the Interplay Between Genes, Social Environment and Human Fertility

\section{PhD Thesis}

to obtain the degree of $\mathrm{PhD}$ at the

University of Groningen

on the authority of the

Rector Magnificus Prof. E. Sterken

and in accordance with

the decision by the College of Deans.

This thesis will be defended in public on

Monday 26 September 2016 at 12.45 hours.

by

Felix Christian Tropf

born on 14 October 1984

in Alzenau, Germany 


\section{Supervisors}

Prof. M. C. Mills

Prof. H. Snieder

\section{Co-supervisor}

Dr. N. Barban

\section{Assessment committee}

Prof. H.-P. Kohler

Prof. M. Murphy

Prof. C. H. Mulder 


\section{Contents}

Introduction to social science genetics \& overview of the studies ...............................

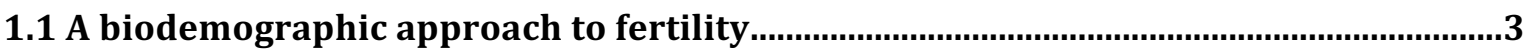

1.1.1 Defining fertility ...................................................................................................................

1.1.2 Why is a socio-genetic approach to fertility less prevalent? .................................................

1.1.3 The foundations: intermediate fertility variables/ proximate determinants ....................6

1.1.4 The emergence of a biodemographic approach to fertility ..................................................

1.1.5 Measuring genetic influence: family studies, twin studies and GREML models................9

1.2 The chapters of this volume in the light of previous genetically informed fertility research

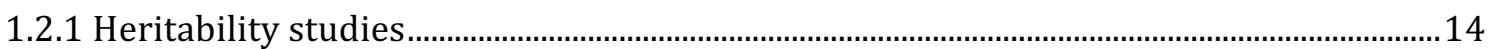

1.2.2 Beyond heritability estimates: GxE interaction and multivariate models of fertility

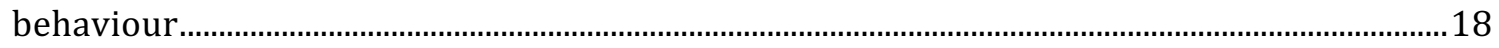

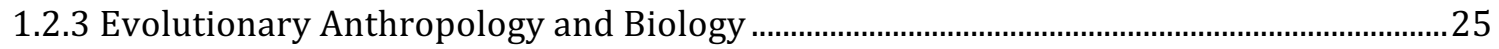

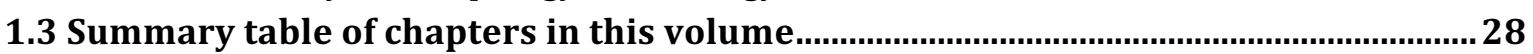

1.4 Conclusions and discussion: Limitations and fertile future research frontiers.........32

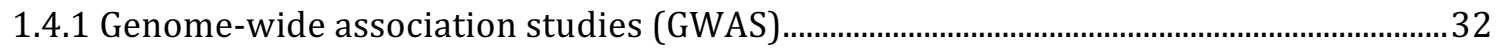

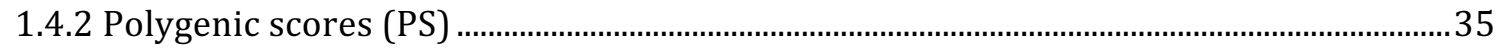

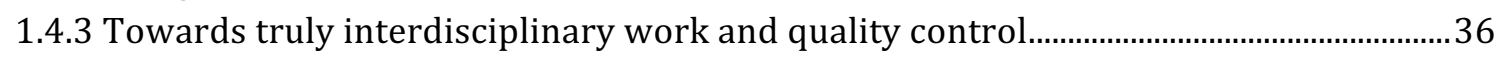

1.4.4 Towards more complex models of gene $\mathrm{x}$ environment (GxE) interaction........................ 37

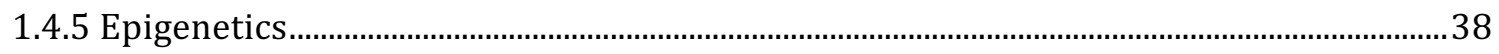

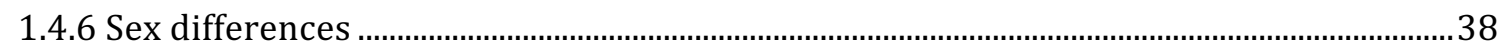

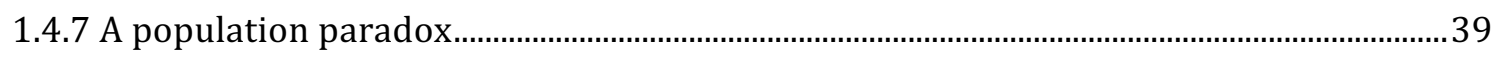

1.4.8 Integrating new data and methods from quantitative genetics and reproductive

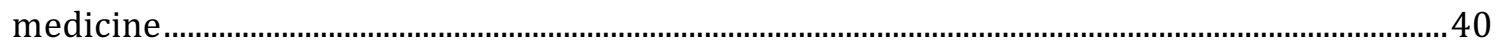

Genetic influence on age at first birth of female twins born in the UK, 1919-68.. 43

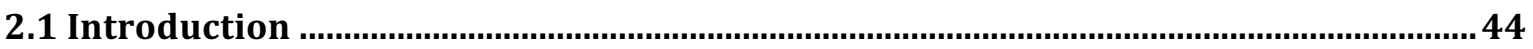

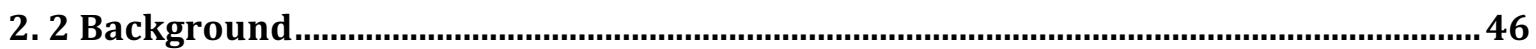

2.2.1 Intergenerational correlations of fertility ……................................................................ 46

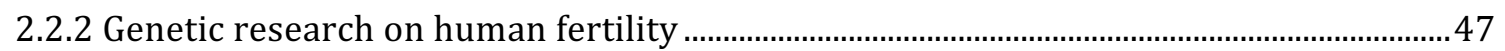

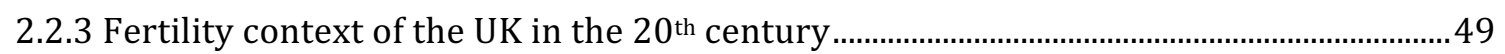

2.3 Data \& method

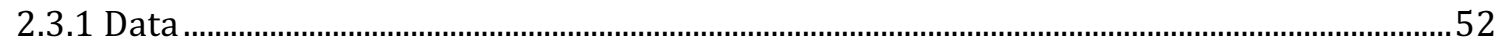

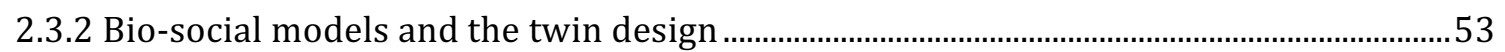

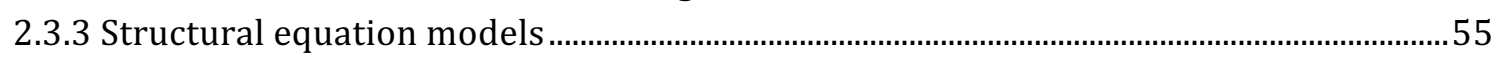

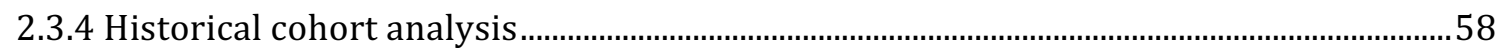

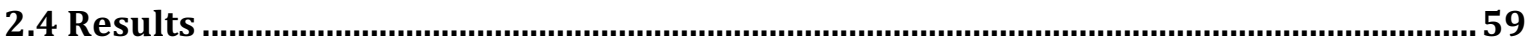

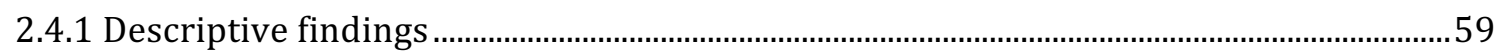

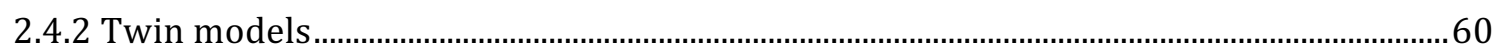

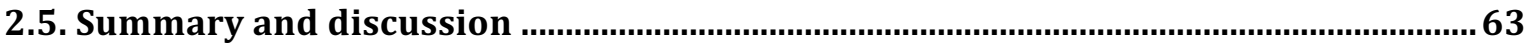

Human fertility, molecular genetics, and natural selection in modern societies.. 71 


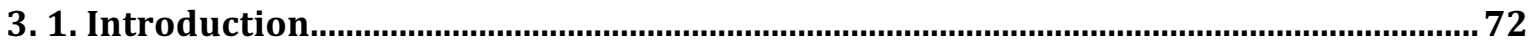

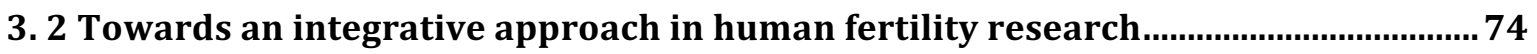

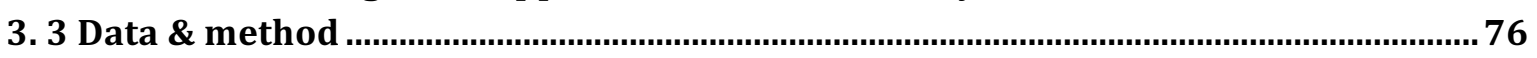

3. 3. 1 Data

3. 3.2 Genotypes

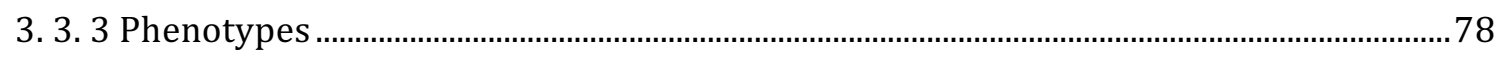

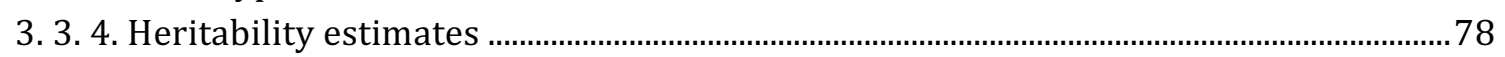

3. 4 Results

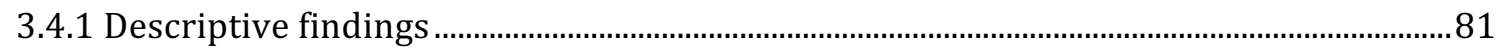

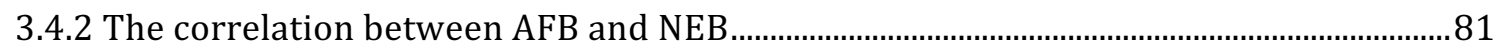

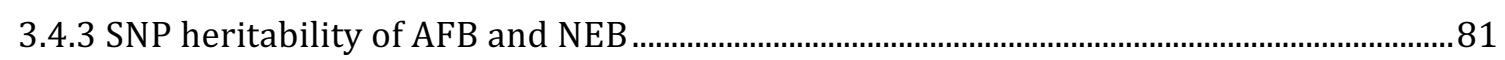

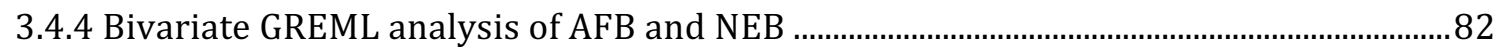

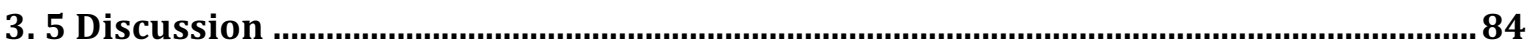

Mega-analysis of 31,396 individuals from 6 countries uncovers strong geneenvironment interaction for human fertility ...................................................... 91

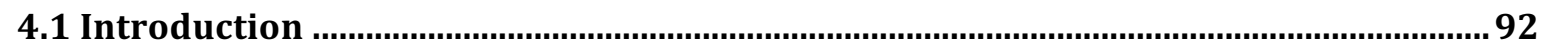

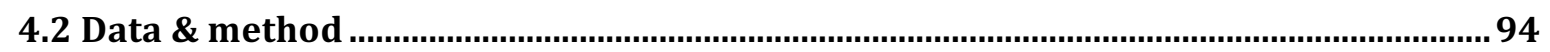

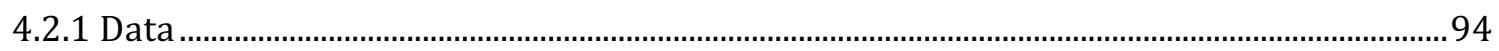

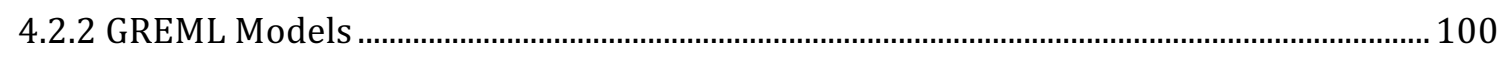

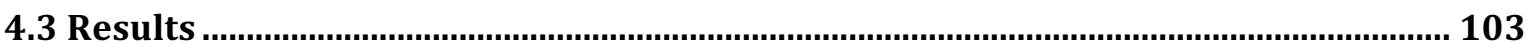

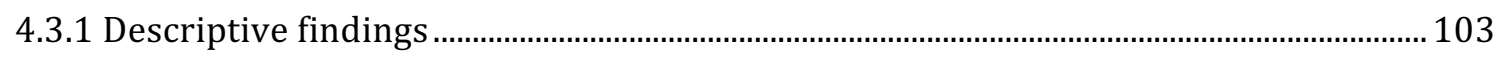

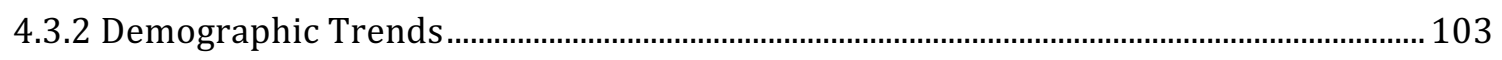

4.3.3 Genetic effects on fertility from the whole genome ......................................................... 104

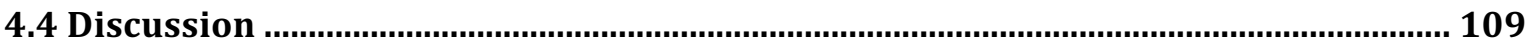

Is the association between education and fertility postponement causal? The role

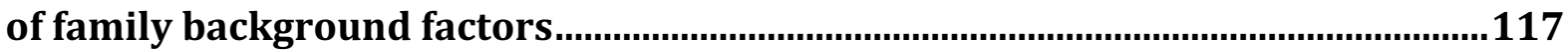

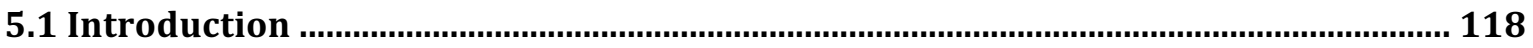

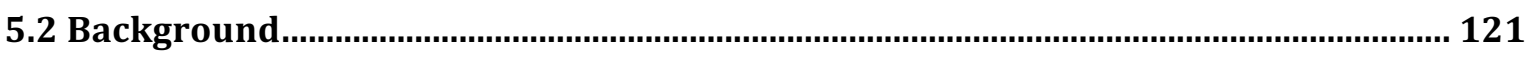

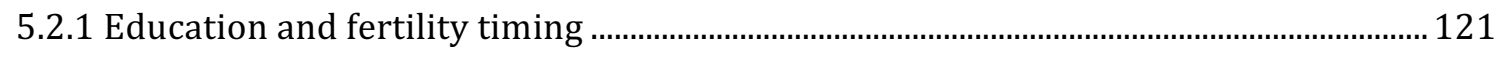

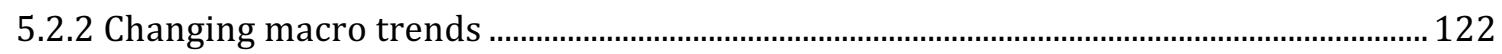

5.2.3 Fertility postponement: Alternative explanations ............................................................ 123

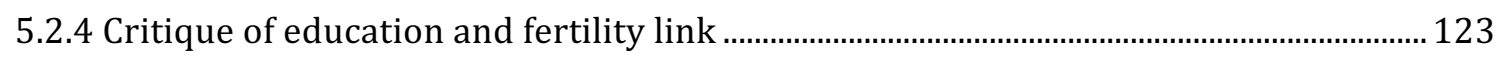

5.2.5 Biometric approaches for the education and age at first birth link ................................ 124

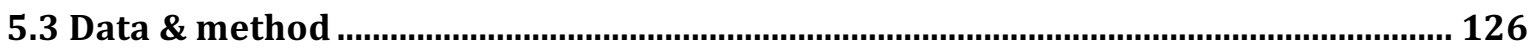

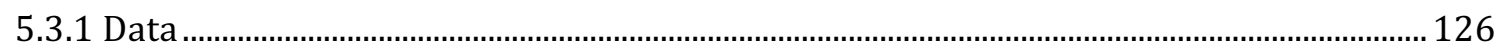

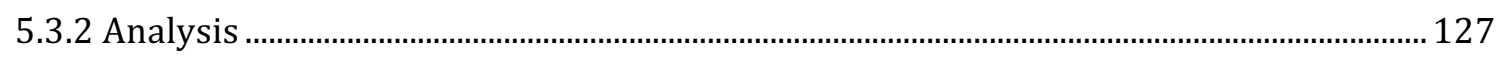

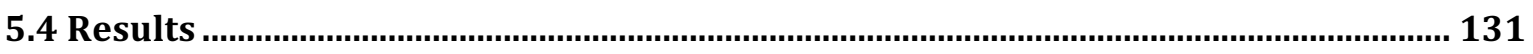

5.4.1 Descriptive findings ....................................................................................................... 131

5.4.2 The causal effect of education on age at first birth .......................................................... 134

5.4.3 The role of genes and the environment as family background factors ............................ 139

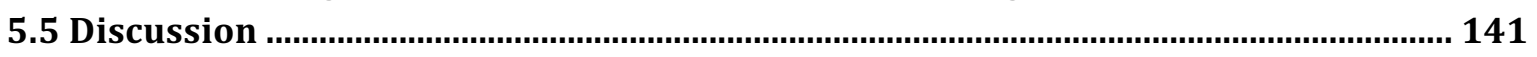

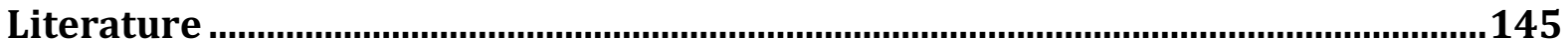


Supporting Information (SI) …….................................................................... clxix

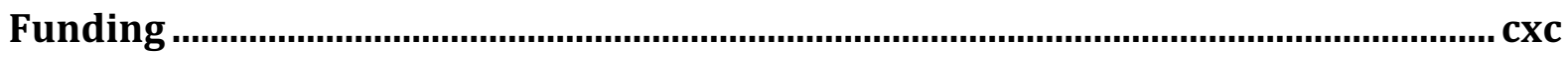

Data availability statement ............................................................................... cxcii

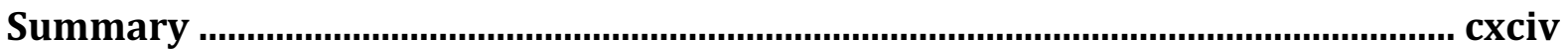

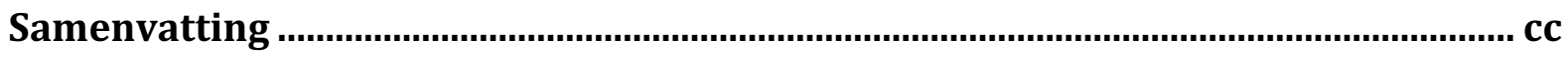

Zusammenfassung.......................................................................................................ccvi

Résumé..................................................................................................................... ccxii

Acknowledgements.......................................................................................... ccxix

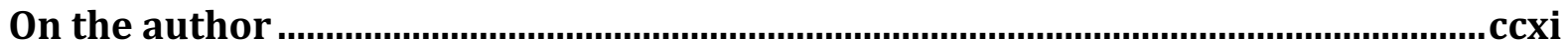

ICS Dissertation Series.......................................................................................... ccxii 





\section{Introduction to social science genetics \& overview of the studies ${ }^{1}$}

Fertility research on human populations has been largely dominated by social science or environmental explanations of fertility behaviour and outcomes (Balbo, Billari, \& Mills, 2013). Yet a growing body of research over the last few decades has demonstrated the relevance of including biological and genetic factors into our understanding of fertility outcomes (Foster, 2000; Kohler, Rodgers, \& Christensen, 1999; Kohler, Rodgers, Miller, Skytthe, \& Christensen, 2006; Kohler, Rodgers, \& Christensen, 2002; Kohler \& Rodgers, 1999, 2003; W B Miller, Bard, Pasta, \& Rodgers, 2010; Rodgers et al., 2008; Tropf, Barban, Mills, Snieder, \& Mandemakers, 2015; Tropf, Stulp, et al., 2015; Udry, 1996). Recent advances in biology, molecular genetics, medical sciences, reproductive medicine and evolutionary anthropology have likewise increased the relevance of adopting an interdisciplinary approach to study fertility.

The aim of this dissertation is to challenge and extend existing knowledge on genetic and environmental influences on fertility, and to provide an interdisciplinary approach across the fields of genetics and social sciences for fertility researchers. The main body of this book consists of four empirical studies, which quantify genetic effects on age at first birth and number of children based on twin data (chapters 2 \& 5) and molecular genetic data (chapters $3 \& 4$ ), as well as the genetic correlation across both outcomes (chapter 3). We investigated patterns of gene-environment interaction across birth cohorts (chapters $2 \& 4$ ) as well as the common base of genetic effects on fertility outcomes across populations (chapter 3). Finally, we used genetically informed statistical models to approach the causal effect of education on the age at first birth as well as its contribution to fertility postponement during the $20^{\text {th }}$ century in the United Kingdom (chapter 5).

1

This chapter is based on Mills, M. C. \& F. C. Tropf (2016). The biodemography of fertility: A review and future research frontiers. In Social Demography Forschung an der Schnittstelle von Soziologie und Demografie (pp.397-424). Springer Fachmedien Wiesbaden. 
felix-tropf.com

This introduction has four goals: first, it provides some background on the history of research into the intersection between social science and biology, in order to make the book accessible to a readership from the social sciences. This review focuses on the contribution from biodemographic research. Biodemography is a new branch of science that integrates biology and demography, focussing on the complementary biological and demographic determinants of and interactions between the birth and death processes insofar as they relate to populations, and often to humans in particular (Carey \& Vaupel, 2005). We first reflect on past challenges that slowed the application of a biodemographic approach to fertility research, and briefly describe the emergence of the field and introduce the basic concepts and terminologies. Second, we provide a review of previous examinations to take stock of what we have learned until now. Our work draws primarily on research carried out within demography and sociology, but with attention to more recent work particularly in the areas of evolutionary anthropology, and behavioural and molecular genetics. We will localize our work in this body of literature to identify the contributions of this dissertation in the larger framework of the scientific field. Third, we provide short summaries of the studies conducted in the chapters of this volume. Finally, we discuss persisting issues of our field of research and propose future frontiers for promising research in social science genetics and fertility in general.

We have opted to organize the scientific history of the biodemography of fertility around several themes. We first review the main reasons for the lack of attention - and in some cases outright resistance - to adopting a integrative approach to fertility research. We then discuss the early foundations of combining a biological and behavioural approach to the study of fertility via the use of intermediate fertility variables. Next, we turn to a review of research that has adopted a behavioural genetic approach to determine whether fertility has a genetic component, often using family and twin study designs. This is followed by more recent research in the area of molecular genetics, which gradually shifts from identifying whether there is a genetic component to fertility to isolating where it is located on the genome. Anthropological and evolutionary approaches are then touched upon. The chapter concludes with a broader discussion that reflects upon 
what we have learned up until now, as well as potential future frontiers in this area of research.

\subsection{A biodemographic approach to fertility}

\subsubsection{Defining fertility}

Before embarking upon this volume, it is useful to first define the terminology related to 'fertility', as it often differs across disciplines. It is essential to note that the broader term fertility has different meanings in demography, evolutionary biology and reproductive medicine (Mills, Rindfuss, McDonald, \& te Velde, 2011). In demography and related social sciences, 'fertility' refers to performance and the bearing and timing of live births. The focus is often on the two interrelated terms of the tempo (or timing) of childbearing and the quantum or actual number of children that women have during a certain period. Throughout this volume, We mainly refer to two of the most central indicators studied in this field until now, which is the tempo measure of age at first birth (AFB) and the quantum measure of number of children ever born (NEB).

In demography and sociology, quantum is often referred to as the number of children (e. g. Kohler et al., 1999) whereas in biological research the same outcome is referred to as life-time reproductive success (Stearns, Byars, Govindaraju, \& Ewbank, 2010) or the number of offspring (Zietsch, Kuja-Halkola, Walum, \& Verweij, 2014). In evolutionary research, fertility quantum may represent a proxy for 'fitness, ${ }^{2}$ which is a function of the number of children of a subject in relation to the number of children of peers of the same birth cohort (e. g. Kirk et al., 2001; Stearns et al., 2010). This in turn is used to measure how far the fertility quantum leads to relatively higher chances to successfully transmit genes to the next generation.

\footnotetext{
2 We are aware that this is a simplification of the term fitness and that more nuanced measures differentiate between absolute fitness (ratio between number of individuals with genotype after selection to those before selection) and relative fitness (average number of surviving progeny of a particular genotype compared to average of surviving progeny of competing genotypes after a single generation). That is, fitness is defined as a propensity or probability of the expected average number of offspring in a particular group or class of individuals and not only the actual number of offspring produced. Additionally, long term measures such as number of grandchildren give a better assessment of reproductive success across generations.
} 
felix-tropf.com

Ideally, fitness of individuals is measured across several generations, however, it has been argued that due to improvements in hygiene and the reduction in prenatal, infant and child mortality in industrialized societies, NEB is a valid proxy for lifetime reproductive success indicating biological fitness-while much more feasible for empirical investigations (Stearns et al., 2010). Complementarily Zietsch and colleagues (2014) found a perfect genetic correlation between number of children and number of grandchildren amongst Swedish Twins, validating this argument. Another distinction between disciplines is the terminology related to fertility versus fecundity. In reproductive medicine, fertility denotes the ability of couples, women or men, to conceive and have children given unprotected intercourse. In demography and sociology, fertility refers to the number of children, while fecundity defines the ability to conceive.

\subsubsection{Why is a socio-genetic approach to fertility less prevalent?}

Although there has been some recognition of the biology underlying fertility, sociologists and demographers have been reticent in adopting and integrating findings and approaches from behavioural and molecular genetics, neuroendocrinology and cross-species life history analysis (Wachter \& Bulatao, 2003). Although not exhaustive, we outline some central reasons for this lack of enthusiasm.

A first historical reason, also noted by others (Kohler et al., 1999; Rodgers, Kohler, Kyvik, \& Christensen, 2001), is the putative implication of Fisher's (1930) Fundamental Theorem of Natural Selection (FTNS). Fisher's theory states that fertility is a fitness trait, which theoretically entails that heritability should be (close to) zero. As we demonstrate shortly, however, a series of studies produced evidence that this is not the case.

A second prominent reason for the paucity of research that adopts a behavioural genetic approach in the social sciences in general - but particularly in relation to fertility - is the dark history related to eugenic policies that emerged in the $1880 \mathrm{~s}$ and subsequent extreme historical atrocities. Eugenics focused on 'improving' humanity via supposedly scientific methods that proposed selective breeding. As Levine and Bashford (Furstenberg Jr., Levine, \& Brooks-Gunn, 1990) describe, the aim of the eugenics movement was "to affect reproductive practice 
through the application of theories of heredity." The aim was to prevent life (sterilization, contraception, abortion), encourage 'fitter' life (training, rearing of children, public health) and promote pro-natalist goals, but also at its most extreme, to end life (so-called euthanasia of the disabled) (Levine \& Bashford, 2010). As a result of these policies, hundreds of thousands of people were segregated and sterilized, or lost their lives. This perspective has been widely, and rightly, condemned. It is essential to note that the type of research described in this volume and within the mainstream of contemporary peer-reviewed research in behavioural and molecular genetics has no eugenic goals or ties. Considering this grave history and link of eugenics and fertility, it remains important to explicitly acknowledge this point with the goal to prevent similar abuses to ever occur in the future.

Third, as noted in a Population Studies article by Thoday et al. (Thoday et al., 1970), social scientists often ignore biology and genetics (and vice versa) due to a lack of understanding and training in their concepts and methods, and to the virtual absence of cooperation between disciplines, which arguably still holds today almost 50 years later. As touched upon shortly, the growth of candidate gene studies in the social sciences came at around the same time that they were shown to be an incorrect method that produced false positives in genetics (Ioannidis, Ntzani, Trikalinos, \& Contopoulos-Ioannidis, 2001). The lack of interdisciplinary research teams and funding has resulted in parallel literatures and disciplines that operate almost entirel independent of one another.

Fourth, although this has rapidly started to change, the survey and registration data mainly used by social scientists to study fertility has generally lacked any biomarker or genetic measures. Conversely, if combined genetic and register or survey data is available, many of the medical or genetic cohort datasets only include very crude measures of core social science and environmental indicators even though they are likely pivotal in understanding gene and socioenvironment interactions (e.g., education, occupational or marital history, social capital and networks).

This reluctance, the lack of data, and the lack of interdisciplinary training that combines strong social and biological or genetic measures, has resulted in fertility theories and explanations in the social sciences that are generally socially deterministic, often solely based on concepts such as agency, motivation, conscious 
felix-tropf.com

choice and intentions (for review see Balbo et al., 2013), which are in turn highly conditioned by the environments of the family, peers, organizations, local and national institutional contexts (Mills \& Blossfeld, 2005). Increasing evidence demonstrates that choice, agency and the behavioural outcomes that we examine in fertility, however, are not only socially-determined, but also linked to an individual's genetic architecture and beyond, such as proteins, hormones, neurons, gametes and other factors (Freese, 2008; Rietveld et al., 2014; Rietveld, Cesarini, et al., 2013; Rietveld, Medland, et al., 2013; Udry, 1996; Wachter \& Bulatao, 2003; Wachter, 2008). Furthermore, recent research demonstrates that - as Udry (1996) and others hypothesized some time ago - a portion of the genetic influence of fertility is related to the motivational precursors to fertility (W B Miller et al., 2010; Rodgers, Kohler, et al., 2001). In other words, integrating genetics into the analysis of fertility will significantly improve our understanding and the explanatory power of our models. It is important to stress that biological explanations and heritability should not be mistaken for genetic determinism. As we describe shortly, the link between genes and phenotypic traits or outcomes may be indirect and mediated through environmental and psychological factors. This review will argue towards an integrative approach across disciplines.

\subsubsection{The foundations: intermediate fertility variables/ proximate} determinants

As a first attempt to integrate biological determinants within contemporary demography, Davis and Blake (Davis \& Blake, 1956) introduced intermediate fertility variables, an approach which was later refined and quantified by Bongaarts (J Bongaarts, 1982; John Bongaarts, 1978) and Bongaarts and Potter (1983) under the label of proximate determinants of fertility. These seven factors that include both biological and behavioural determinants have been highly influential in shaping our theory and understanding of human fertility in demography - while remaining an exception of biodemographic work at that time. These are: (1) proportion of married women among all women of reproductive age (as a proxy to capture women exposed to sexual intercourse, also more broadly measured later by percentage of women in a sexual union, frequency of sexual intercourse), (2) contraceptive use 
effectiveness, (3) duration of postpartum infecundability (or postpartum insusceptibility), $^{3}$ (4) induced abortion, (5) fecundability, (6) prevalence of permanent sterility; and, (7) spontaneous intrauterine mortality. The first four were considered to be the most important determinants due to the fact that they differ greatly between populations and the fact that fertility is the most sensitive to changes in these measures.

Criticisms and revisions of the model acknowledged the need to include sexual activities outside of marriage and a revised definition and estimate of total fecundity (Stover, 1998). Hobcraft and Little (Hobcraft \& Little, 1984) also extended this work with their focus on fertility exposure analysis. These determinants were considered to be theoretically strong and highly plausible candidates for explaining the relationship between the level of fertility and both biological and behavioural factors. Yet further research using individual-level micro data demonstrates that additional factors also operate to explain the variation in fertility outcomes and levels (for review see: Balbo et al., 2013; Mills et al., 2011).

\subsubsection{The emergence of a biodemographic approach to fertility}

Recognition of the importance of biological or genetic determinants underpinning demographic behaviour in this area of research began to flourish in the late 1990s. Biodemography in general grew as a fruitful interdisciplinary approach, first applied in the area of longevity and mortality studies and the relationship between fertility-longevity interactions (Vaupel et al., 1998; Wachter, 1997, 2008).

Udry (1994; 1996) promulgated some of the earliest calls to include a biodemographic approach to study fertility and related behaviour in contemporary demographic research. His landmark article in 1996 went beyond the data constraints of that period and the application of simple behaviour-genetic models (Plomin, 1994) to hypothesize a series of probable biosocial relationships at the individual micro-level and societal macro-level. This included consideration of

\footnotetext{
3 Bongaarts (1982) adapted the definition of postpartum infecundability to represent the combined effect of postpartum amenorrhea and abstinence.
} 
additive, indirect and interaction effects between biological (hormonal, genetic) and social factors and environments. He likewise acknowledged that the behavioural choices or motivations to have children were likely guided by biological predispositions such as genetics, hormones, neurological structure or neurotransmitters. Such predispositions could be examined by proxy by personality for example, which has been shown as an antecedent to childbearing motivations and fertility outcomes (W B Miller et al., 2010; W B Miller \& Pasta, 1994; W B Miller, 1992; Warren B Miller, 1994).

Another pivotal hypothesis introduced in Udry's (1996) paper outlined how changing social arrangements could alter the proportion of variance in fertility behaviour that is biologically controlled. In other words, in times when there are high normative and social constraints on the 'proper' timing of first birth and number of children a woman or couple should have, the less the variance in their social behaviour should be controlled by biological forces. Conversely, in the period of the second demographic transition where individuals and couples are less socially constrained and have considerable choice, biological forces should have a stronger influence on behaviour. Indeed, Udry's argument implies that biological predispositions should be more important now than in the past. This idea was adopted in further research to understand cohort differences, which we describe shortly (e. g. Briley, Harden, \& Tucker-Drob, 2015; Kohler, Rodgers, et al., 2002; Kohler et al., 2006).

At the start of the 2000s, the biodemographic approach to fertility began to flourish with two interdisciplinary books appearing, Offspring: Human Fertility Behaviour in Biodemographic Perspective, co-edited by Wachter and Bulatao (2003) and The Biodemography of Human Reproduction and Fertility co-Edited by Rodgers and Kohler (Rodgers \& Kohler, 2003). Both books provided broad coverage of the topic that goes beyond this limited review (e.g., endocrinology, neuroscience) and remain essential reading for this topic. Perhaps the most influential research and approach in fertility until now, however, has been the application of behavioural genetics models, which we turn to now. 
1.1.5 Measuring genetic influence: family studies, twin studies and GREML models

The first questions to be answered in all behavioural genetics research are whether and to what extent genes have an influence on a trait ${ }^{4}$ of interest (Guo, 2005). In other words, it is the basic question first asked by Kohler, Rodgers and Christensen in 1999: 'Is fertility behavior in our genes?' where they adopted a twin study approach to first ask and examine this question directly in demography, the findings of which will be described shortly.

The statistical concept used to measure genetic influences on a trait within a population is called heritability. Heritability is a population parameter (Visscher, Hill, \& Wray, 2008) quantifying in how far differences between individuals are due to genetic differences. It is defined as the proportion of the genetic variance $\left(V_{G}\right)$ over the overall variance of the trait $\left(V_{P}\right.$ phenotype) in a specific population (for details see Visscher 2008):

$$
H^{2}=\frac{V_{G}}{V_{P}}
$$

Whereas the phenotypic variance is the sum of genetic and environmental variance.

$$
V_{P}=V_{G}+V_{E}
$$

The genetic component can be furthermore differentiated into additive $\left(V_{A}\right)$ genetic effects-when the contribution of an allele to a phenotype is fixed-and nonadditive ( $V_{N A}$, epistatic and dominant) effects-when the contribution of an allele to the phenotype depends on the presence of other alleles or genes.

$$
V_{G}=V_{A}+V_{N A}
$$

$H^{2}$, defined as the genetic variance over the total variance in an outcome represents so called broad sense heritability. Generally, for most quantitative traits a trait that shows continuous variation (Turkheimer, 2000) - it is assumed genetic variance is mainly due to additive effects . For fertility and other quantitative traits, empirical evidence for epistatic or dominant effects is negligible (Kohler et al., 2006;

\footnotetext{
4 This research often refers to a trait or phenotype, which in the social sciences is generally referred to as an outcome or dependent variable.
} 
Zhu et al., 2015). Therefore, the genetic component underlying a trait is commonly quantified based on additive genetic effects and so called narrow sense heritability and when heritability is used in this volume it means:

$$
h^{2}=\frac{V_{A}}{V_{p}}
$$

In other words, heritability quantifies the additive genetic variance component of an outcome variable.

It has been argued that all traits are heritable to some extent (Turkheimer, 2000) and that heritability is ubiquitous in social science (Freese, 2008). However, a number of caveats are in order. First, the genetic variance component provides insight into the extent to which genetic variation in a population is associated with variation in fertility, and a recent meta-analysis of all twin studies published up to 2012 has documented considerable variation in heritability across traits (Polderman et al., 2015). Second, fertility is a particularly interesting trait to study, because FTNS predicts that due to natural selection, there would be no heritability for traits such as NEB (Fisher, 1930). Nevertheless, we find a genetic component underlying fertility and it has been argued that the interplay of genes and the (social) environment is responsible for that (Hughes \& Burleson, 2000; Kohler et al., 1999; Udry, 1996; chapters $2 \& 4$ are focussing on gene-environment interaction). Heritability can be estimated without measuring actual genes. For this reason, family (Bras, Bavel, \& Mandemakers, 2013) and most commonly twin studies (Snieder, Wang, \& MacGregor, 2010; see also chapters 2 \& 5) are conducted. With the advent of molecular genetic data, a new analytical technique based on the genetic relatedness of unrelated individuals has become prominent (Visscher, Yang, \& Goddard, 2010; Yang, Lee, Goddard, \& Visscher, 2011; see also chapters 3 \& 4; Yang et al., 2010), and we discuss these approaches in the next section.

\section{Family Studies}

Traditional family studies often follow simple designs and study e.g. parentoffspring correlations (Fisher, 1930; Pearson, Lee, \& Bramley-Moore, 1899). Parents and children share on average 50 percent of their genes, so that two times the observed correlations in a trait, for example number of children ever born (NEB), 
estimates the heritability assuming that the shared environment of family members does not play a role for. Correlations in fertility are very common among family members and also increased during the Twentieth century (Murphy, 1999). It should also be noted that additional fertility studies in demography have examined the impact of genes albeit indirectly via the study of the intergenerational transmission of the number of children (Anderton, Tsuya, Bean, \& Mineau, 1987; Murphy \& Wang, 2001), teenage pregnancy (Kahn \& Anderson, 1992) and age at first birth (e. g., Barber, 2000; Steenhof \& Liefbroer, 2008).

The pioneering work of Fisher (1930) found an intergenerational correlation of 0.20 in the NEB in data from around 2,000 British aristocrats born at the end of the Nineteenth century. He therefore estimated that 40 percent of the variance in the NEB within this population was attributed to genetic differences. The assumption that intergenerational correlations in fertility are entirely due to genetic effects, however, does not likely hold. In fact, we know from numerous studies in sociology and demography that shared environmental factors among family members or nongenetic (or cultural) inheritance of traits can lead to similarities in fertility among family members (e. g., Murphy, 1999; Steenhof \& Liefbroer, 2008). The educational level or economic status, for example, are relatively stable across generations (Jæger, 2012; van Doorn, Pop, \& Wolbers, 2011) and at the same time important for fertility outcomes (Mills et al., 2011). Therefore, it is necessary to separate genetic from environmental effects in families and within different societal contexts.

\section{Twin Studies}

The most common way to disentangle influences of latent family factors on any trait of interest is to use the information available in twin data. Twin models follow a relatively simple and straightforward logic and until now, represent one of the best resources for evaluating the importance of genetic variation in observed traits (Boomsma, Busjahn, \& Peltonen, 2002).

Twin models facilitate the comparisons between two kinds of twins, identical or monozygotic $(\mathrm{MZ})$ and fraternal or dizygotic (DZ) twins, in order to quantify genetic and non-genetic (or environmental) influences. In a classic twin design (as it is applied in chapters 2 and 5), MZ as well as DZ twins are siblings of virtually 
felix-tropf.com

identical ages and born and raised in the same families. The siblings are consequently assumed to share common environmental influences such as their parents, the neighbourhood they grew up in and other related aspects to the same extent, which is called the equal environment assumption. More importantly, MZ twins are genetically identical (i.e., share all their genotypes). DZ twins in contrast, are akin to full siblings and thus share on average only around 50 percent of their segregating genetic material. Similar to parent-offspring correlations, the correlation among DZ twins therefore reflects the importance of both environmental and genetic effects in families. The degree, however, to which MZ twins have a higher correlation in the trait of interest than DZ twins, reflects the degree to which they are genetically more similar. The comparison of twin correlations thus already makes it possible to quantify genetic and shared environmental effects on a particular trait (Boomsma et al., 2002; Snieder et al., 2010).

Since both kind of twins are assumed to share family environment to the same extent and $M Z$ twins are twice as similar as DZ twins with regard to their genes, narrow sense heritability is two times the difference of the intra-group correlations of $\mathrm{MZ}$ and $\mathrm{DZ}$.

$$
h^{2}=2 *(\mathrm{r}(\mathrm{MZ})-\mathrm{r}(\mathrm{DZ}))
$$

The effect of the common environment of the twins $\left(c^{2}\right)$ is therefore the pairwise correlation of MZ minus the heritability.

$$
c^{2}=\mathrm{r}(\mathrm{MZ})-h^{2}
$$

Variance that is unexplained by these factors is due to non-shared environmental effects $\left(e^{2}\right)$ from outside or even within the family; including measurement errors: for details (see Snieder et al., 2010).

Based on this logic, structural equation modelling (SEM) has become standard in twin research (as applied in chapters 2 \& 5; see Boker et al., 2011 for details; Neale \& Cardon, 1992). Above correlations have low power and large standard errors and do not make use of information available in variances and covariances. SEM furthermore provides goodness of fit statistics enabling the testing of alternative models and identifying assumptions - for example whether there are dominant genetic effects (Snieder et al., 2010). Extensions of the twin model to other relatives as well as other family designs are possible. 
There has been some criticism of the potentially problematic assumptions and practical limitations of twin studies (see also chapter 2; for critical discussions on the equal environment assumption for example, see Horwitz et al. 2003). Both twin and family designs are likewise limited for other reasons in fertility research such as the fact that they require a pair of siblings, thus excluding singleton children and thus individuals from low fertility families. Since dizygotic twinning also has a genetic basis (Hoekstra et al., 2008; Mbarek et al., 2016), it is likely that these twins carry genes that might be important for high fertility. Thus, there might be a non-random genetic stratification which could bias variance estimates. Finally, these designs require multiple data on family-members, which are more difficult and costly to gather than data on unrelated individuals. With the advent of molecular genetic data, a new analysis technique has been developed to quantify genetic effects on a trait, namely genomic-relationship-matrix restricted maximum likelihood (GREML) models, which have been applied in chapters $3 \& 4$ and are discussed below.

\section{GREML models}

The GREML analyses (as applied in chapters 2 \& 5) follow the same logic as the twin models. However, heritability estimates are based on the genetic similarity between pairwise unrelated individuals who do not share the same micro environment. To achieve this, a pairwise genetic similarity matrix is estimated from measured molecular genetic information across the entire genome. The approach capitalizes on recent developments in microarray technology to identify the association between specific traits and all common genetic variants across the whole genome. Such a design is possible due to the completion of the Human Genome Project in 2003 and the International HapMap Project in 2005 which provided the basic tools to find the genetic contributions of traits. As with other genetic data available until now, it is necessary to have the DNA from each participant in the study, often via a blood or saliva sample. Each person's DNA is then placed on tiny microarray chips and scanned on automated laboratory machines. These machines 
quickly overview each person's genome for strategically selected markers of genetic variation, referred to as SNPs (single nucleotide polymorphisms ${ }^{5}$ ).

The GREML analyses correlate the genetic similarity with the phenotypic similarity between individuals. More precisely, based on a mixed linear model, it performs a restricted-maximum likelihood (REML) analysis to decompose the observed variance of an outcome into genetic and residual variance (environmentally caused and measurement error). The method has first been applied to height as a model complex trait (Visscher et al., 2010; Yang et al., 2010). Chapters 3 and 4 exploit these (quite) recent advances in the field of molecular and quantitative genetics. Note that in contrast to genome-wide association studies (GWAS, see 1.4.1) GREML methods do not aim to identify individual genetic variants associated with an outcomes, but to quantify the genetic variance explanation of an outcomes based on all common SNPs from the whole genome.

\subsection{The chapters of this volume in the light of previous genetically informed fertility research}

This section summarises a series of studies that have focussed on estimating the heritability of NEB and AFB, using the aforementioned behavioural genetics approach. Gene and environmental interaction at the macro-level is then touched upon, concluding with multivariate models that go beyond heritability estimates and a brief mention of topics related to motivation and education. We localize and detail the contributions of the chapters in this volume in the light of the literature (for an overview of the chapters in this volume see Table 1-1, section 1.3).

\subsubsection{Heritability studies}

Several twin and family studies provide evidence for a genetic component underlying both the tempo (AFB) and quantum (NEB) of human fertility up to a

5

DNA consists of around 3 billion pairs of nucleotide molecules and each can be indexed by its location on the genome. There is no variation for most of these nucleotides across individuals; rather, we differ in our polymorphisms and hence the focus on SNPs, which occur once every 100 to 300 nucleotides. A single gene can contain hundreds of SNPs. 
level of 50 percent (e. g. Byars, Ewbank, Govindaraju, \& Stearns, 2010; Kirk et al., 2001; Kohler et al., 1999; Milot et al., 2011; Neiss, Rowe, \& Rodgers, 2002; Nisén, Martikainen, Kaprio, \& Silventoinen, 2013; Tropf, Barban, et al., 2015; Zietsch et al., 2014). Figure 1-1 provides an overview of selected studies, which investigated the heritability of fertility behaviour across birth cohort. The figure shows the estimates for women for age at first birth (AFB) and for both men and women of number of children ever born (NEB). Here we can see that heritability estimates for AFB for women range between 0.001 (USA 1920-1935 and 1956-1970) and 0.35 (UK 19301939) or in other words that up to just over 0 or 35 percent of the observed variance in AFB within these birth cohorts is due to additive genetic effects. NEB in women ranges from 0.01 (USA 1920-1935) to as high as 0.59 (USA 1936-1955) and for men, the range for the same trait is between 0 (Denmark 1880-1900) and 0.56 (Netherlands 1810-1820). The figure shows that there is considerable variation in heritability estimates across time, between countries and the sexes, a point we return to later.

\section{Heritability of AFB}

The age at first birth (AFB) has been assessed in a recent study by Nisén, Martikainen, Kaprio, \& Silventoinen (2013) on Finnish twins born between 1950 and 1957. For men they found that common environmental factors between siblings and not their genes - mainly explain resemblance in the AFB among brothers. For women, in contrast, they estimate heritability of the AFB at 0.26 , which is also in line with studies from Australia (Kirk et al., 2001) and the U.S. (Byars et al., 2010 as well as our findings in Chapter 2). However, the AFB turns out to be a challenge to study, since other studies, e.g. in the U.S. (Neiss, Rowe, \& Rodgers, 2002) and Denmark (Rodgers et al., 2008), show no significant effect on the AFB of women.

Chapter 2 in this dissertation investigates for the first time genetic effects on the AFB among twins from the UK born 1919-68. A core issue in the analysis of AFB is the handling of right-censored observations (i.e., individuals that did not have a first birth by the last time of observation). In contrast to the commonplace practice of including right-censored cases in demography and sociology (e.g., Mills 2011), it is uncommon to deal with censored information in genetic studies and childless women are generally excluded from the sample (Byars et al., 2010; Neiss et al., 2002; 
felix-tropf.com

Nisén et al., 2013; Rodgers et al., 2008). Using data from the TwinsUK cohort (Moayyeri, Hammond, \& Spector, 2013), Chapter 2 empirically examined whether the inclusion of censored information in a Tobit model affects heritability estimates compared to the classic models. Results show strong and non-systematic differences between both kinds of models suggesting that the comparison of these research designs has to be critically reconsidered. The Tobit model estimates - in line with previous studies from Australia and Finland - that a substantial part (40 percent) of the variation in AFB is associated with latent family characteristics. Genetic dispositions (26 percent) play a more important role than the shared environment of siblings (14 percent), with the nonshared environment/measurement error having the strongest influence (60 percent).

In Chapter 3, we extended this analysis and applied the recently developed GREML method, which is based on molecular genetic data and uses the genetic similarity of unrelated individuals, who share no micro environment to estimate the heritability of AFB. Given that this method makes use of only the fraction of genetic variance amongst unrelated individuals, large sample sized are required and we combined data from the TwinsUK with the Lifelines Cohort Study (Klijs, Scholtens, \& Mandemakers, 2015) to increase statistical power. The analysis based on around 5,000 individuals estimates SNP-based heritability to be 0.15 , meaning that common SNPs explain 15 percent of the observed variance in the AFB across individuals in the combined sample.

\section{Heritability of NEB}

Focusing on NEB, a study by (Rodgers, Kohler, et al., 2001) investigated genetic and environmental influence on the NEB among female and male twins from Denmark born between 1953 and 1959. They found that twin correlations among MZ pairs $(\sim 0.30)$ are more than twice as high as those of DZ twin pairs $(0.13)$ for both males and females. The structural equation models estimate heritability for both sexes at around 0.30 , meaning that 30 percent of the observed variance in the NEB within these birth cohorts is due to additive genetic effects. Both non-additive genetic and shared environmental effects were not significant, and therefore the remaining 70 percent of the variance can be attributed to non-shared environmental 


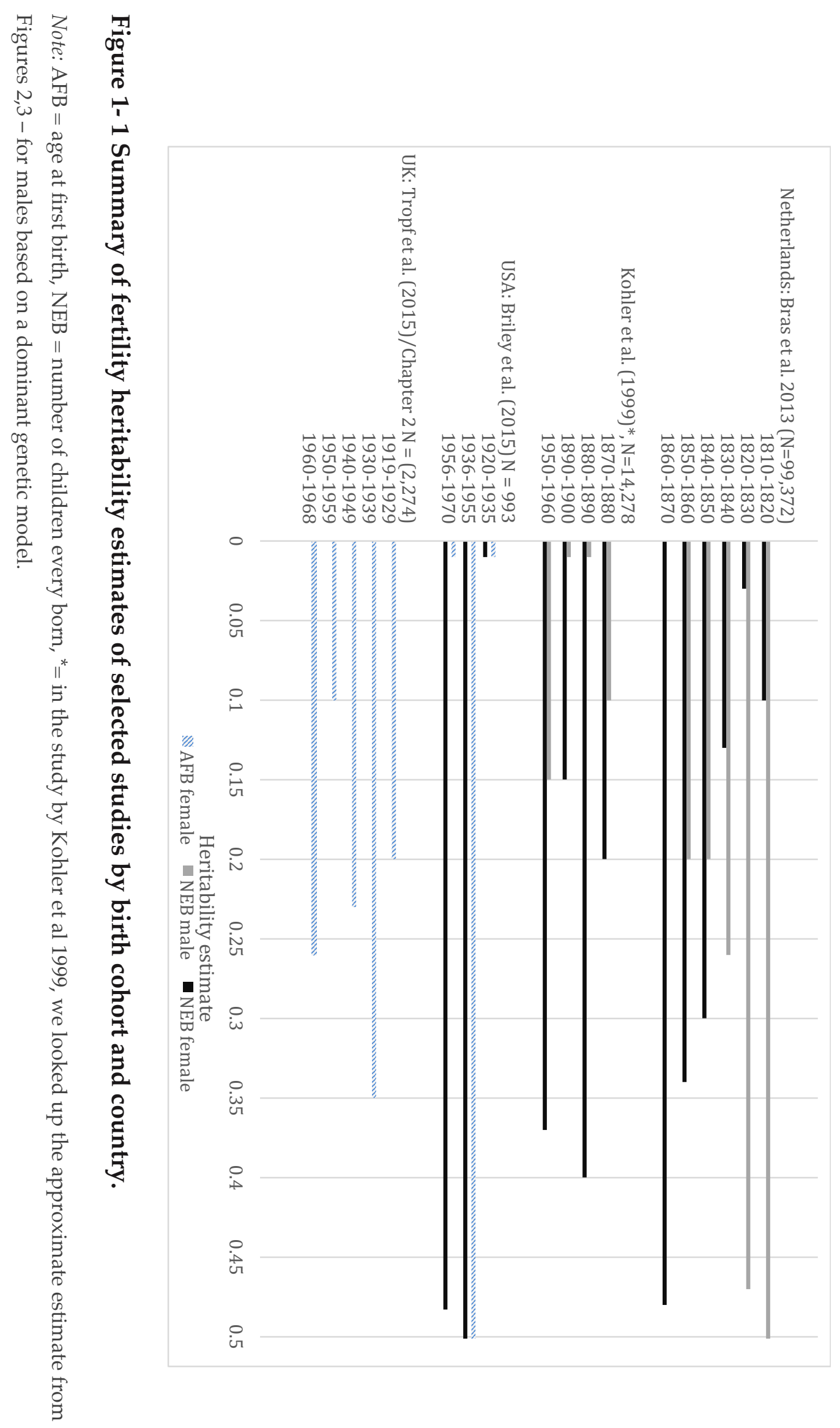


felix-tropf.com

effects between the siblings, which also includes measurement error. These findings are almost perfectly replicated in a recent study of Swedish twins born between 1915-1929 (Zietsch et al., 2014). Not only fertility research, but behavioural genetic research in general shows that considerable variation is attributable to non-shared environmental factors. Unique environmental factors appear to be the main source of variation; amongst others, the partner of the individuals plays an important role for fertility behaviour (Kohler \& Rodgers, 2003).

In chapter 3 of this dissertation, we also investigated genetic influence on NEB in the the GREML model. We found that 10 percent of the variance in the NEB is explained by genetic factors - as for AFB, this is smaller than typical estimates from twin models. In general, it is not unusual that heritability estimates obtained by GREML utilizing molecular genetic data are lower compared to estimates from family or twin studies. Yang, Manolio et al. (2011) observed that such common SNP heritabilities cover one third to one half of the total heritability found in twin and family studies with the remaining genetic variance most likely explained by lower frequency variants (Visscher, Brown, McCarthy, \& Yang, 2012; Yang et al., 2015). Another possibility is that non-additive genetic factors (Zhu et al., 2015; Zuk \& Hechter, 2012) and/or gene-environment interaction inflate heritability estimates from twin studies (see chapter 4).

\subsubsection{Beyond heritability estimates: GxE interaction and} multivariate models of fertility behaviour

Gene-environment interaction $(\mathrm{GxE})$ refers to the situation where genetic effects that are associated with a trait are dependent upon the variability in the environment or when genes modify an organism's sensitivity to particular environmental features (e. g. Shanahan \& Hofer, 2005). A particular context can trigger or compensate for a genetic vulnerability and improve adaptation. In general differences in heritability estimates between birth cohorts and countries apparent in Figure 1-1 may suggest gene-environment interaction in reference to fertility. These findings suggest that biodemographic research on fertility needs to continue to embrace demographic and sociological research, because different heritability 
estimates for a trait between different populations do not necessarily point purely to methodological issues, but can be informative in substantive terms.

As described previously, researchers such as Udry (1996) hypothesized that there is likely an interaction of biological with societal level factors at the macro- or population level. In fact, one of the most common misconceptions about genetic studies is that the heritability of a trait within one population can predict the heritability in the same trait in another population - even though similarities in estimates can be remarkable for physical traits such as height (Visscher et al., 2008). First, heritability is a ratio of genetically caused variance over the overall variance. Therefore, changes in the overall variance in a trait can change heritability independent of the genetic variance component, or in other words - heritability is a population specific parameter. Second, the genetic variance component depends on the genetic endowment, for example, of allele frequencies within a population. Third, and presumably most important for fertility, genes always only encode predispositions for a trait, but environmental conditions determine whether these dispositions become manifest (Guo, 2005). Thus, genes and environment can interact and replication in different temporal and spatial settings is pivotal to gain insight about this interplay.

Gene and environment (GxE) interaction in fertility across populations and birth cohorts

Gene $x$ environment (GxE) interaction has been examined in the study of NEB. Using data on the historical Danish twin cohorts including virtually every twin pair born since 1870, Kohler et al. (1999) and Kohler, Rodgers, \& Christensen (2002) report large changes in heritability estimates of the NEB for cohorts born during the past centuries. Particularly, after the strong fertility decline of the First Demographic Transition at the end of the nineteenth-century and the Second Demographic Transition in the second half of the twentieth-century, heritability reached a moderate level of 0.40 - while getting close to zero in the interim phases.

As described in more detail previously, this fits with the explanation hypothesized by Udry (1996) who described changes in the influence of genes on reproductive behaviour in terms of GxE with factors such as societal norms 
constraining choice and behaviour. Udry argued that genetic predispositions for fertility gain importance in environments that are less restrictive in their fertility choice. At the same time, genetic predispositions play a minor role in restrictive social contexts such as strong normative rules about the timing and number of children, war or economic crisis.

Following Udry's (1996) hypotheses, Kohler et al. (1999) associated the observed peaks in heritability with the environmental changes during the Demographic Transitions. In particular, there were improvements in economic, medical and hygienic conditions during the First Demographic Transition, with the Second Demographic Transition characterized by the introduction of the pill and cultural transformations relaxing fertility norms triggering genetic expressions (van de Kaa, 1987). A recent study corroborates this reasoning, applying an extension of the family model on a large scale database containing 100,000 sibling pairs from the Dutch Province of Zeeland born between 1810-1870 (Bras, Van Bavel, \& Mandemakers, 2013). These findings not only show for women a rise in heritability during the first Demographic Transition, but they present evidence that this rise was especially pronounced for women in more urban areas or with a liberal religious climate, thus underscoring the idea that individual freedom triggers genetic influences on the NEB.

In Chapter 2 of this dissertation, we analysed changes in the genetic influence on the AFB across birth cohorts from 1919-1968 of female UK twins. We also found large swings in heritability co-varying with strong environmental upheavals. While heritability is low or non-significant for birth cohorts who started childbearing during the end of World War II or the economic crisis of the 1970s and 1980s, it rises to around 0.40 for individuals who started childbearing in times of changing mores and the sexual freedom in the 1950s and 1960s. These results appear to be well in line with previous studies on both NEB (e. g. Bras et al., 2013; Kohler et al., 1999) and AFB (Briley et al., 2015), confirming the initial rationale of Udry (1996).

It is important to note that Udry's heuristic assists interpretation of the observed trends in heritability estimates over time and across groups. However, estimates from twin studies typically have large standard errors, and while differences across time (Kohler et al., 1999; Tropf, Barban, et al., 2015) or religious groups (Bras et al., 2013) are in the expected direction, they remain mostly non- 
significant. Additionally, previous studies have reported findings of substantial heritability (0.30-0.50) in traditional, pre-transitional societies (Milot et al., 2011), for which the FTNS and Udry's theory would predict low heritability. Ongoing and future research based on molecular genetic data, particularly polygenic scores (see Conley \& Domingue, 2016; Conley et al., 2016 for applications), will permit testing of specific hypothesis in statistically more powerful ways that twin- and family studies.

In chapter 4, we went one step further than than twin studies and investigated whether the same genes are important for NEB and AFB in different geographical and temporal environments. We conducted a mega-analysis of individual level data-in contrast to a meta-analysis of summary statisticsapplying whole genome methods on 31,396 unrelated men and women from six Western countries (Australia, Estonia, Netherlands, Sweden, UK, US). Across all individuals and environments, common SNPs explained only $\sim 4$ percent of the variance in NEB and AFB. We then extended these models to test whether genetic effects are shared across different environments or are unique to them. For individuals belonging to the same population and demographic cohort (born before or after the $20^{\text {th }}$ century fertility decline), SNP-based heritability was almost five times higher at 22 percent for NEB and 19 percent for AFB. We also found no evidence suggesting that genetic effects on fertility are shared across time and space. Our findings imply that the environment strongly modifies genetic effects on the tempo and quantum of fertility, that potentially ongoing natural selection is heterogeneous across environments, and that gene-environment interactions may partly account for missing heritability in fertility.

\section{Multivariate modelling}

Although the focus until now in this review has been on NEB and AFB, other related fertility traits have also been examined. Genetic influences have been explored for the age at first dating or marriage (Mealey \& Segal, 1993), age at first sexual intercourse (Dunne et al., 1997), number of sexual partners (Guo, Tong, \& Cai, 2008) and likelihood of unprotected sexual intercourse (Daw \& Guo, 2011). One of the strongest genetic effects was found in a twin study examining the first attempt to 
felix-tropf.com

have a child among the Danish twins, measured in retrospective interviews of the first attempt to conceive. In this study, up to 50 percent of the variance in that trait was explained by genetic differences (Kohler et al., 1999; Rodgers et al., 2001). This raises the question of how different fertility traits relate to each other and to what extent the same genes or the same environments are important for different fertility traits.

Several studies engage in more complex (twin) modelling approaches (e.g., Kohler, Behrman, \& Schnittker, 2011) estimating, for example, bivariate genetic models. Following the same logic as in classic twin studies, it is possible to estimate the extent to which the same genetic and environmental factors are important for two different traits. If trait 1 of twin 1 predicts trait 2 of twin 2 , the correlation of traits runs in families and therefore there is a common etiology of the traits within the family. If this cross-trait-cross-twin correlation is higher for $\mathrm{MZ}$ twins than for DZ twins, the common etiology is partly genetic. If the genetic correlation between two traits is 1 , all genetic variance in trait 1 and 2 has a common base. If the genetic correlation is 0 the genetically based variance between trait 1 and 2 are independent. For a more detailed empirical explanation, refer to Kohler et al., (2011).

\section{The age at first birth and number of children ever born}

Two studies from Australia (Kirk et al., 2001) and the US (Byars et al., 2010) have used twin and family designs to investigate whether genetic influences on the AFB and NEB have a common base. Both studies find that the well-established negative correlation between AFB and NEB is partly genetic, meaning that genes that are associated with a later AFB are also associated with a lower NEB across the life-course. In chapter 3, we also investigated whether genetic effects on AFB and NEB are correlated, but did this using bivariate GREML models, which produce unbiased estimates of shared genetic effects amongst unrelated individuals (S. H. Lee, Yang, Goddard, Visscher, \& Wray, 2012; Visscher et al., 2014). In the aforementioned sample of women from the Netherlands and UK, we find a statistically significant genetic correlation between AFB and NEB of -0.62 ( $S E=0.27$, p-value $=0.02$ ), implying that a considerable part of the underlying genetic factors are shared between AFB and NEB. 


\section{Decision-making and fertility motivations}

One area of research has focused on decision-making and motivations. Rodgers et al. (2001) combined information on the NEB in the Danish twin cohorts with the first attempt to become pregnant. They found firstly, that the age at first attempt to conceive is heritable for both men (0.35) and women (0.52) and secondly, that around 10 percent of the genetic variance in the NEB is related to the genetic variance in the first attempt to conceive. This study can be seen as key evidence that part of the genetic dispositions influencing our realized fertility is not due to physical traits, but mediated by conscious decision making.

A more recent study by Miller et al. (2010) empirically demonstrated that a portion of the genetically influenced fertility behaviour is related to the motivational precursors to fertility. Examining both timing and NEB and using the NLSY79 in the U.S., they conducted both uni- and multivariate behavioural genetic analyses. The central finding was that there are additive genetic effects that operate through desires to have a certain number of children that affect both the timing of the next child that is born and the ultimate NEB. They also link these affects to gender role attitudes but find no genetic variance associated with either child-timing intentions or educational intentions. This was an extension of previous work such as that by Miller (1994) and Pasta and Miller (2000), showing that both positive and negative childbearing motivations were significantly heritable and that the fertility outcomes we observe are results of prefernces. This builds on Miller's (1994) Traits-DesiresIntentions-Behaviour (TDIB) theoretical framework.

\section{Education, fertility and causality}

Education - and particularly women's education - is a central predictor of particularly the timing of first and subsequent births (Mills et al., 2011). Multivariate genetic models have been applied to explore the relationship between education and fertility with respect to its common genetic and environmental base. 
The aforementioned study on Finnish twins (Nisén et al., 2013) shows that the negative association of education with the chance of having any children among women is largely genetically based. The same reasoning accounts for the positive association between education and the chance to have any children for men, suggesting that different genes are important for men and women. Using more complex models, two studies from the U.S. (Neiss et al., 2002) and Denmark (Rodgers et al., 2008) test whether education mediates the positive relationship between intelligence and AFB of women in a behavioural genetic framework. Results show that education partially mediates the effect of intelligence on AFB in standard models, but that this mediation is not significant once a model is fitted that takes latent family factors (environmental and genetic ones) into account. In other words, differences in intelligence, education and the AFB emerge from variance between families, not differences between siblings within the same family.

Such findings may cast doubt on the assumption that education causally affects the timing of childbearing and thus has the potential to challenge the widely accepted assumption that educational expansion of women is the main explanation for the massive postponement in the AFB during the second half of the Twentieth Century (Balbo et al., 2013; Mills et al., 2011; Ní Bhrolcháin \& Beaujouan, 2012). In chapter 5, we focus on this question and apply within-twin regression models as well as multivariate behaviour genetics models on the association between education and age at first birth in a large sample of twins from the UK. Within-twin models include fixed effects per family, which capture all (observed and unobserved) factors shared among the twins - including genetic effects. The estimated effect of education on the age at first birth can therefore be interpreted as a causal effect (for a critical discussion see Amin, Behrman, Kohler, Xiong, \& Zhang, 2015; Boardman \& Fletcher, 2015). Kohler and colleagues (2011) combined this approach with the behaviour genetics structural equation models (the so called ACE-beta model), to investigate simultaneously the causal effect of education on fertility and the effect of genes and the shared (social) environment of siblings on the education-fertility link.

Our findings show that one year of additional schooling is associated with about half a year later age at first birth in standard models. This fell to only 1.5 months for the within-identical twin model that controls for all shared family 
background factors (genetic and family environmental). The ACE-beta model revealed that mainly influences of the family environment - not genetic factors cause spurious associations between education and age at first birth. Lastly, we demonstrated using data from the Office for National Statistics that only 1.9 months of the 2.4 years of fertility postponement for birth cohorts 1944-1969 could be attributed to educational expansion based on these estimates. We conclude that (the rise in) educational attainment alone cannot explain differences in fertility timing (between cohorts).

\subsubsection{Evolutionary Anthropology and Biology}

The behavioural genetics modelling of human fertility has obviously not been an exclusive interest of social scientists, but has also been widely applied by evolutionary biologists and anthropologists. The question of evolution was initially more of a question of surviving until one reproduces, since only those who survived were able to transmit their genes to the next generation. However, due to improvements in hygiene and the subsequent reduction in prenatal, infant and child mortality, whether and how many children an individual bears has become are important question in understanding evolution. If genetic variants are associated with fertility success, this means that some genetic variants will be more frequent than others in subsequent generations, therefore indicating natural selection and evolution (Stearns, Byars, Govindaraju, \& Ewbank, 2010).

This line of reasoning, however, theoretically predicts that fertility as well as other behaviour important for the transmission of genes to the next generation show low or no significant genetic variation (Kimura, 1958) within a population, because evolution should have already trimmed the differences over time. As mentioned at the start of this review, it has been assumed that this so-called Fundamental Theorem of Natural Selection (FTNS, Fisher, 1930) is the reason why genetically informed fertility research has been neglected for many decades (Kohler et al., 1999; Rodgers et al., 2001).

However, empirically this FTNS turns out to be only partly true. On the one hand, we do observe a much higher heritability for morphometric traits (for example height: 0.80 in humans) than for traits such as fertility (Visscher et al., 2008). On the 
felix-tropf.com

other hand, fertility traits do still show significant heritability. Kirk and colleagues (Kirk et al., 2001), for example, analysed the heritability of the NEB to determine to what extent genes influence the relative reproductive advantage in a population of Australian female twins. They find a heritability of 0.36 , indicating that modern societies are still under natural selection.

\section{Evidence for natural selection}

The genetic correlation of a trait with NEB furthermore indicates whether a specific trait is under natural selection. Findings from the Framingham Heart Study, for example, suggest that the same genes influencing the NEB are negatively correlated with a number of traits, among others height, cholesterol levels, systolic blood pressure and the AFB (Byars et al., 2010). Consequently, the authors expect selective changes in the disposition for these traits for subsequent generations (e.g., that the female US population under study will shrink in body-size).

The negative association between NEB and the AFB leads to a prediction of a decrease in the AFB across generations (Byars et al., 2010; Kirk et al., 2001). Such findings are in line with studies on natural populations such as from Milot and colleagues (2011). They observed in a historical population of natural fertility (i.e., the population of ile aux Coudres with high fertility norms in a stable environment) in Canada that the AFB decreased by around 4 years within the past 140 years as a response to natural selection. Kirk and colleagues (2001) argue that in contemporary times, as compared to the past, more psychological and social traits are important for NEB and AFB, and are therefore assumed to be under natural selection. A recent investigation by Stulp and colleagues (2015) shows that in the Netherlands, height in men was consistently positively associated with NEB throughout the past century, which may partly explain the fact that the Dutch are the tallest people on earth.

Our findings of additive genetic variance in NEB (chapter 3 ) thus also suggest the potential for on-going natural selection on AFB. The genetic correlation between AFB and NEB furthermore suggests that women with a predisposition for an earlier AFB will have had more children and therefore more frequently passed on their genes to the next generation. From an evolutionary perspective, we would expect a shift in the AFB to younger ages in more recent birth cohorts. However, what we observe is the exact opposite, namely, a massive postponement in the AFB in most 
recent birth cohorts across developed societies (Balbo et al., 2013; Mills et al., 2011). The same has been reported for educational attainment. While the negative genetic correlation between educational attainment and fertility in the US would predict a decrease in educational attainment on the population level across generations, the average years spent in education have steadily increase throughout the past century (Beauchamp, 2016). It is therefore important to acknowledge that environmental influences such as the introduction of the 'pill' or political reforms are much more relevant for trends in behavioural outcomes such as fertility than natural selection might be (for a detailed discussion see Courtiol, Tropf, \& Mills, 2016). 


\begin{tabular}{|c|c|c|c|c|c|c|}
\hline & 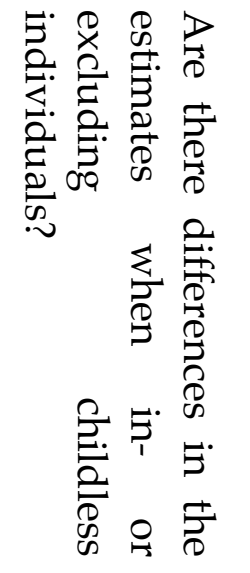 & 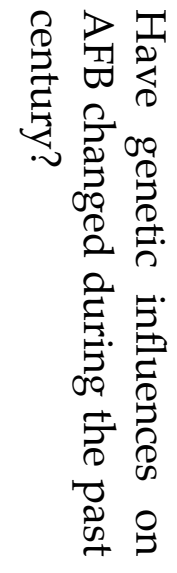 & 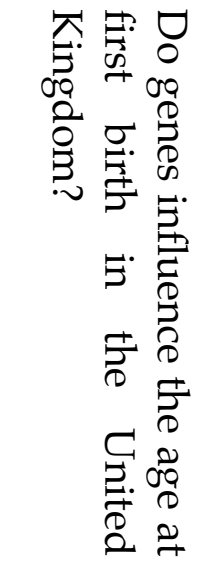 & 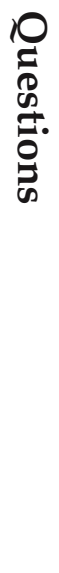 & \multirow{4}{*}{ 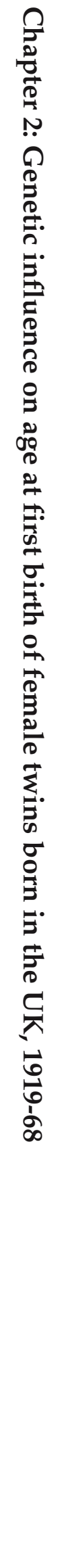 } & \multirow{4}{*}{ 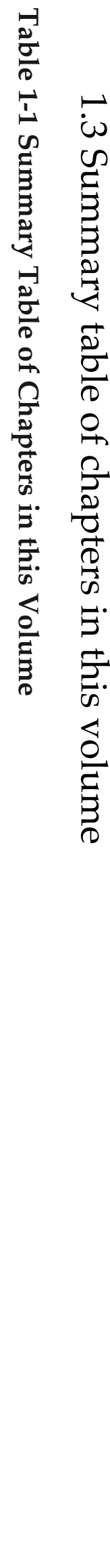 } \\
\hline त्] & 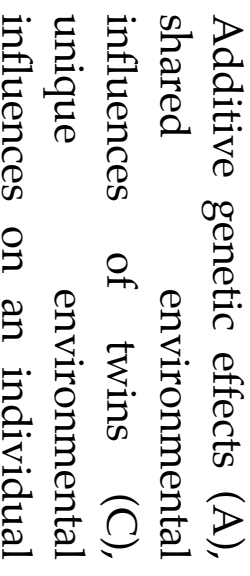 & $\begin{array}{l}7 \\
0 \\
0 \\
0 \\
0 \\
0 \\
0 \\
0 \\
0\end{array}$ & 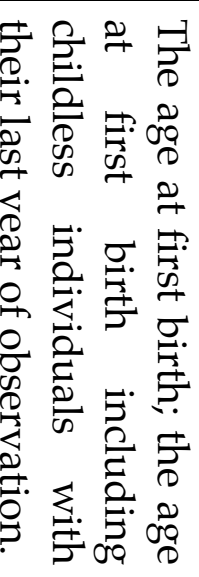 & 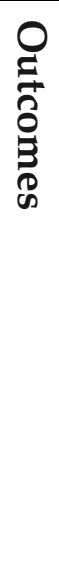 & & \\
\hline & 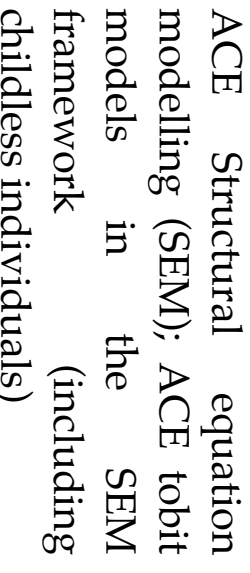 & $\begin{array}{l}3 \\
\frac{3}{2} \\
\stackrel{2}{0} \\
0 \\
\text { के }\end{array}$ & 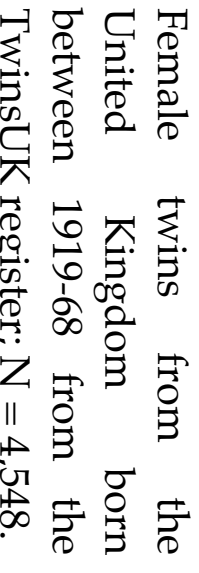 & $\begin{array}{l}\nabla_{D} \\
\frac{D}{2}\end{array}$ & & \\
\hline 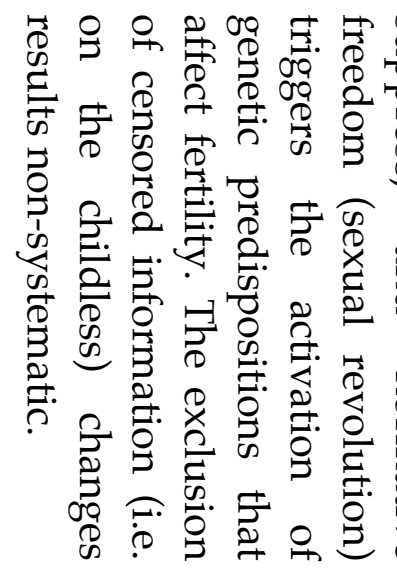 & 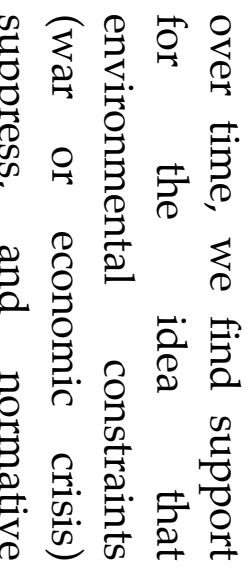 & 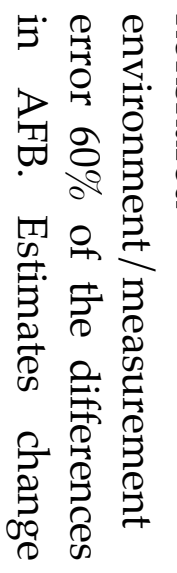 & 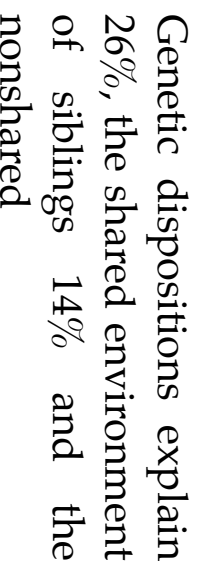 & $\underset{\infty}{\stackrel{\nabla}{\mathscr{D}}}$ & & \\
\hline
\end{tabular}




\begin{tabular}{|c|c|c|c|c|c|c|c|}
\hline & 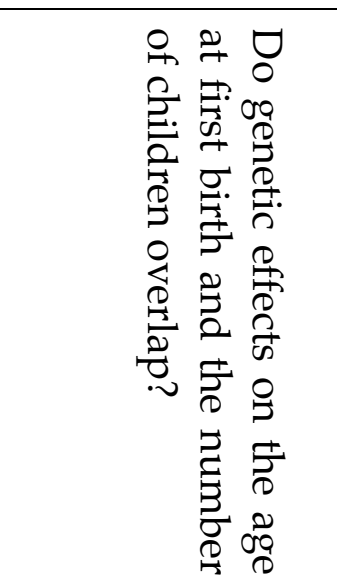 & 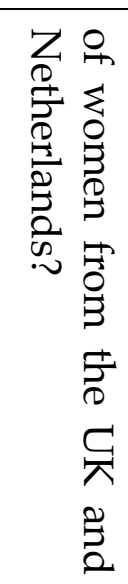 & 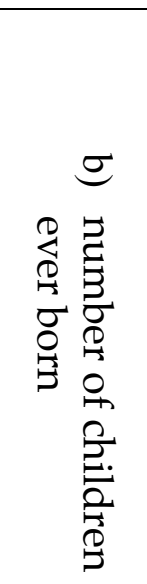 & 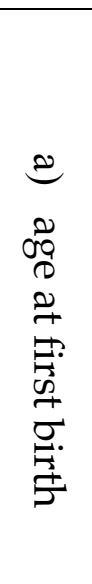 & 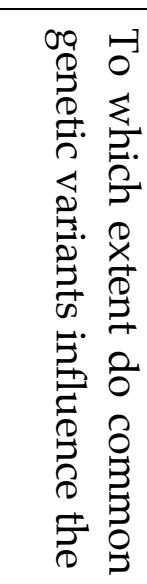 & 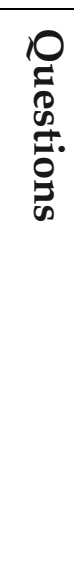 & 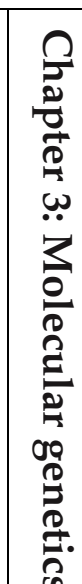 \\
\hline & 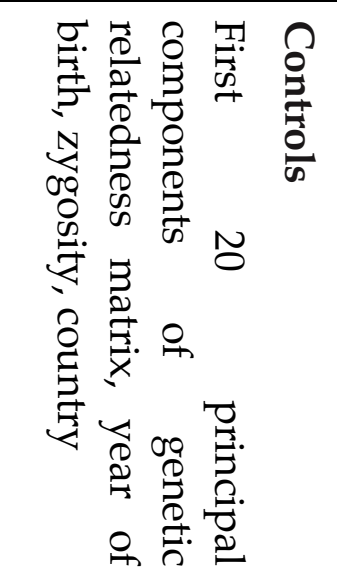 & 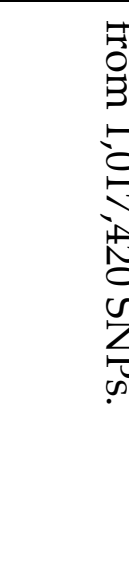 & 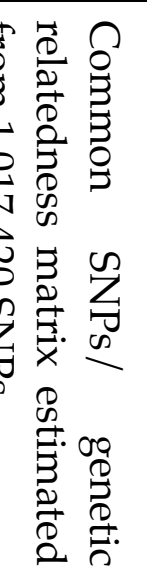 & 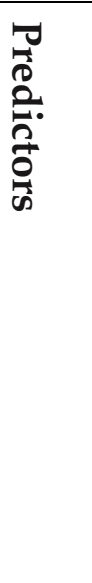 & 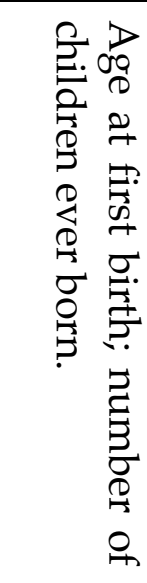 & 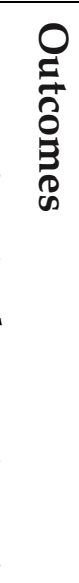 & 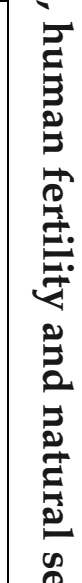 \\
\hline & 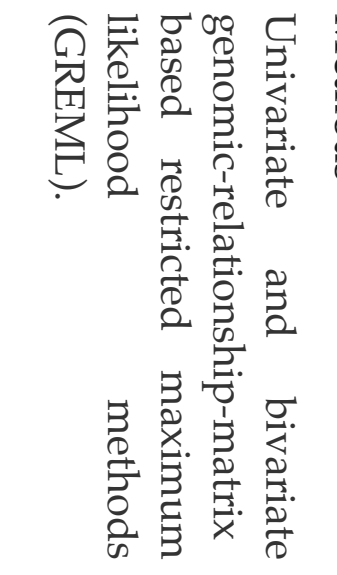 & $\begin{array}{l}3 \\
\frac{3}{0} \\
\frac{1}{0} \\
\frac{0}{2} \\
2\end{array}$ & 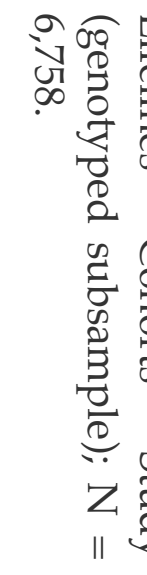 & 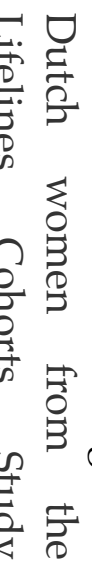 & 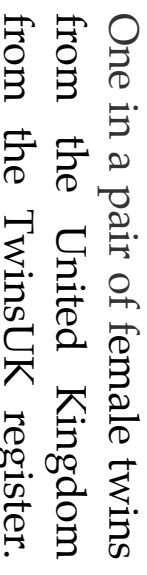 & \begin{tabular}{l}
$\nabla$ \\
\multirow{\Xi}{*}{} \\
\end{tabular} & 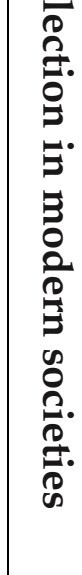 \\
\hline 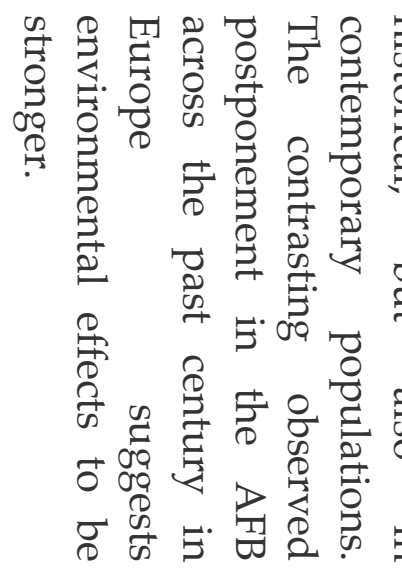 & 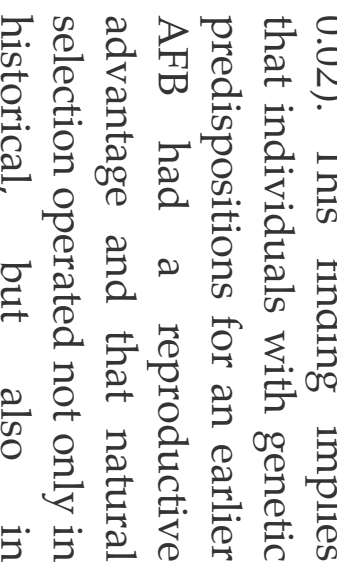 & 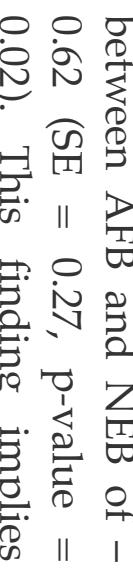 & 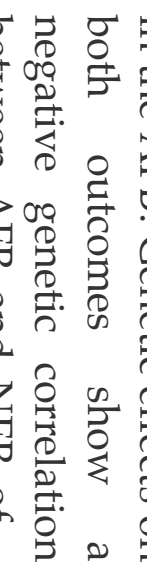 & 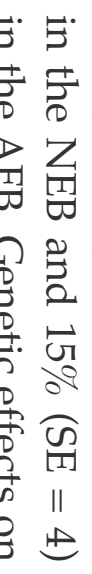 & 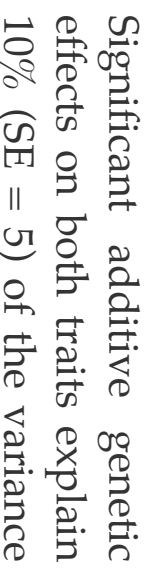 & 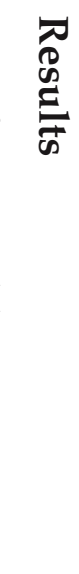 & \\
\hline
\end{tabular}




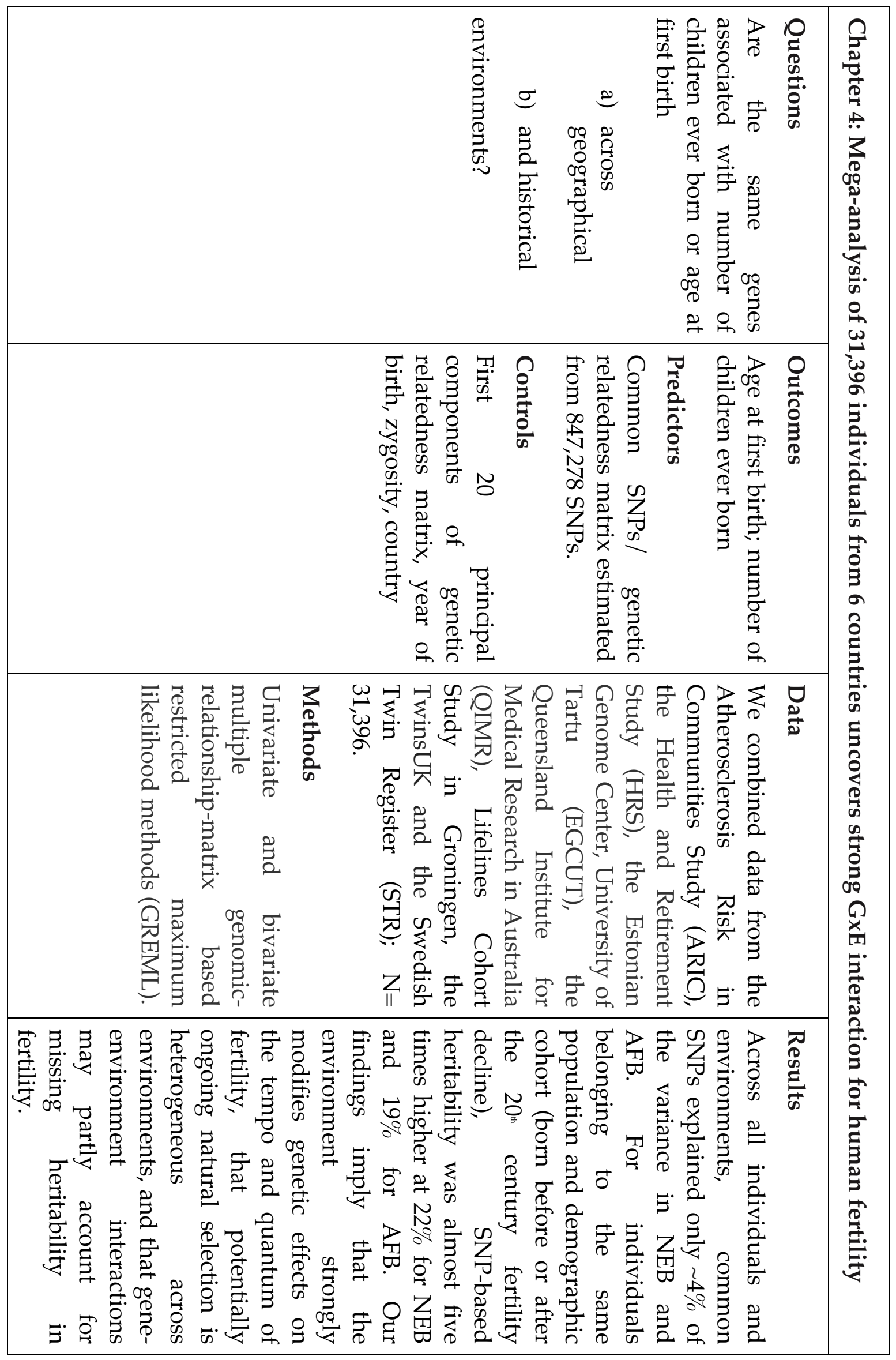




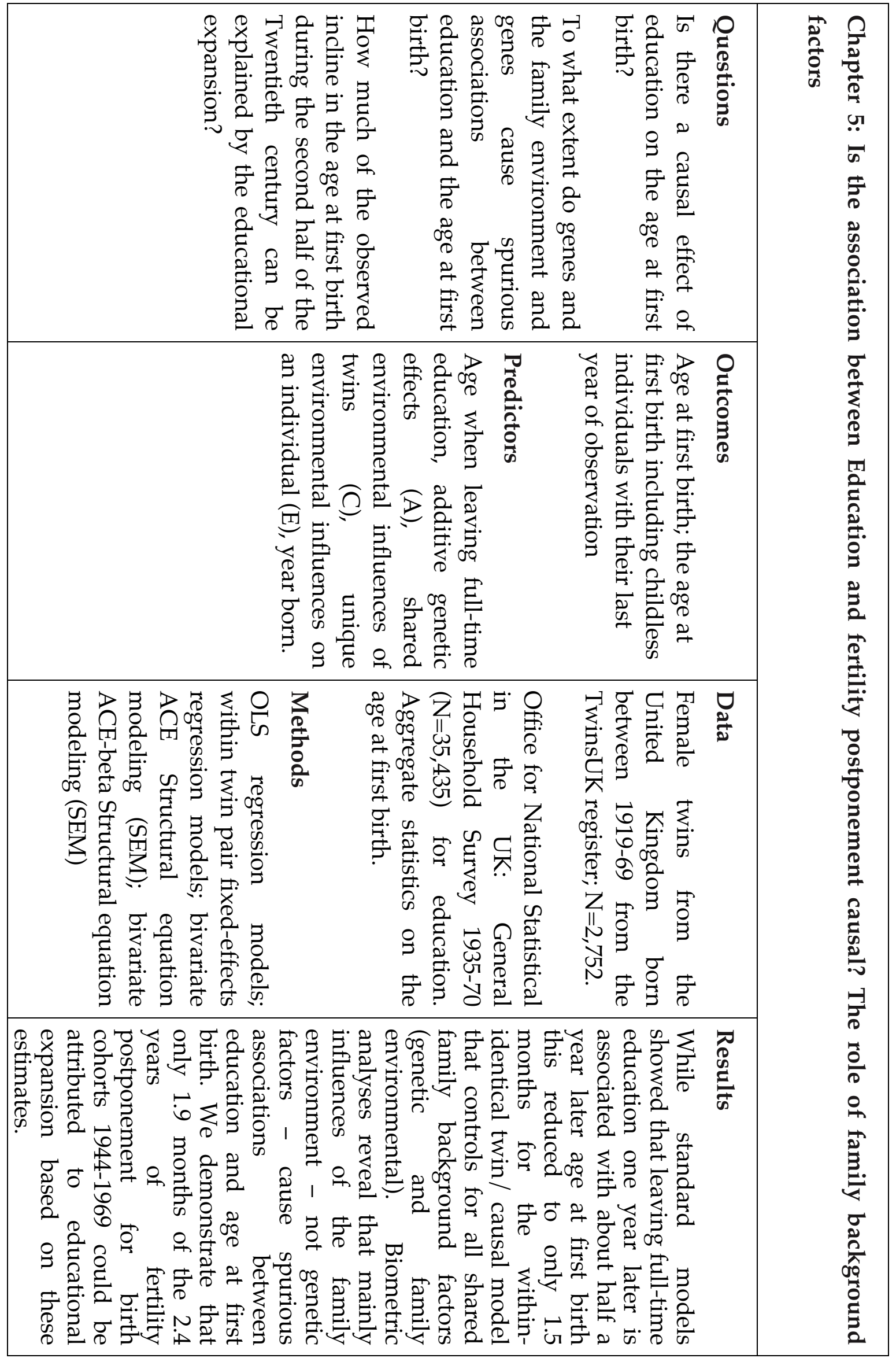




\subsection{Conclusions and discussion: Limitations and fertile future research frontiers}

The aim of this introductory chapter was to examine the current state of knowledge and place the contributions of this book into context withing the biodemography literature. After touching upon the different terminology and underlying reasons for the lack of research into this area, we turned to the foundations of approaches that included both behavioural and biological determinants of fertility. The current review summarized findings from behavioural genetic research on this topic which show in twin and GREML models that there is clearly a genetic component to fertility outcomes, with both AFB and NEB having a heritability ranging from zero to levels of just over 40 percent (as examined in chapters $2 \& 3$ ). Since heritability is a population parameter, heritability of a trait within one population cannot predict the heritability within another (see Visscher et al. 2008, see chapter 4 for a demonstration). This leads us to acknowledge the importance of the environment and the promise of studies that have examined swings in heritability with environmental changes and choice in fertility decisions. Other fruitful directions are studies that have gone beyond heritability estimates to probe into more complex multivariate models of fertility behaviour such as first attempts to conceive a child (Rodgers et al. 2001), motivational precursors to fertility (Miller 1994; Pasta \& Miller 2000; Miller et al. 2010) and the (causal) relation between education and the AFB (see chapter 5).

\subsubsection{Genome-wide association studies (GWAS)}

One central contribution of this dissertation was to introduce molecular genetic data to the study of age at first birth and number of children ever born (chapters 3 \& 4). Virtually all of the fertility-related research conducted within biodemography had adopted a behavioural genetics twin approach until now. The main advantages of using molecular genetic information and GREML approaches 
are: first, that heritability estimates are unbiased by potential gene-environment correlations in families; and second (chapter 4), that we were able to relate the genetic material of individuals across countries and birth cohorts to investigate whether genetic effects on age at first birth and number of children ever born are the same across environments.

Recent advances in molecular genetics, however, offer new possibilities. Simply put, the main distinction is as follows: whereas the models we presented focused on whether fertility is in the genes and to what extent it is heritable, molecular genetics may attempt to isolate where the specific genetic variants responsible are located and subsequently focus on the function of those genes identified.

Advances using a molecular genetic approach first adopted a candidate-gene approach, which has been criticized and requires further replication, but provides an initial window into possible mechanisms and approaches that might be interesting to pursue in future research (Daw \& Guo, 2011; Guo, Tong, \& Cai, 2008; for critique see Hewitt, 2012). More promising approaches are hypothesis-free methods to find genetic variants related to AFB and NEB via a Genome Wide Association Study (GWAS). Since it is highly implausible that even the best social scientist could specify the biological pathway and isolate particular genetic variants in advance, this approach offers an alternative.

GWASs emerged as a promising new approach to connect genetic variants to the outcomes of interest (Zhao \& Chen 2013). GWAS refers to hypothesis-free testing without any a priori assumptions about either the biological pathway or a particular location (Nolte et al. 2010). It likewise embraces the fact that there are multiple genes (polygenic) and pathways associated with fertility that are difficult to specify in advance with our current state of knowledge. A GWAS therefore runs millions of separate regressions on the phenotype (outcome) of interest across the entire genome. Due to the large number of SNPs that are tested in GWAS, an association must achieve a stringent threshold of statistical significance $\left(\mathrm{P}<5 \times 10^{s}\right)$ in order to be considered valid. An association identifies a genomic region and not a specific causative mutation that might be involved in the development of the trait or behaviour. 
The computational GWAS approach remains promising for social science genetics due to the fact that it overcomes some of the mistakes inherent in candidategene studies in the past and due to the fact that the often elusive biological pathways underlying the trait do not need to be specified in advance. It is also the only technique currently available that has the potential to discover novel genes which could then be used in further more reliable follow-up studies and provide directions to researchers where they need to search and which potential biological pathways to pursue. It also allows population stratification to be controlled, which remains a key issue in avoiding bias and misinterpretation of results in this type of research (see Wray et al. 2013 for a review).

A promising first application in the social sciences examined genetic variants that predict educational attainment on more than 120,000 genotyped individuals by Rietveld, Medland et al. (2013) as part of the Social Science Genetics Association Consortium (www.ssgac.com). At the time of writing this review, Mills and her research team at the University of Oxford are currently leading a large consortium to engage in the first ever GWAS and meta-analysis of reproductive choice (age at first birth; number of children), conducted in both men and women in over 60 data sets.

Although GWAS are able to narrow down where to look in the genome, recent research has retorted (Hirschhorn 2009) that the validated SNP associations explain only a small portion of the genetic variance or heritability of the phenotype that has been estimated from classic family and twin studies; this is often referred to as the 'missing heritability' problem (Manolio et al., 2009). Despite the fact we are currently working towards isolating SNPs robustly associated with reproductive behaviour in the previously mentioned GWAS, we show in chapter 4, based on the same type of molecular information utilized in GWAS that this is not the entire story of the genetic architecture of reproductive outcomes. Instead, for a complex outcome such as reproductive choice, we will be able to explain some - but not all - of the genetic heritability with a GWAS. Instead, it appears that the interaction of genes with the environment are likewise crucial.

Although no GWAS has been published as of yet, among the reproductive choice variables we often study in demography and sociology, several studies have shown promising results for fertility-related outcomes related to infertility and the 
reproductive life span (age at menarche, age at menopause). Previous research has successfully demonstrated that there is a genetic component to reproduction with over 70 genome-wide association studies (GWAS) published for 32 traits and diseases associated with reproduction (see Montgomery et al. 2014). This includes identification of genes such as those related to age at menarche (Elks et al., 2010; Sulem et al., 2009), age at menopause (Day, Ruth, \& Thompson, 2015; He et al., 2009; Perry et al., 2009; Snieder, MacGregor, \& Spector, 1998; Stolk et al., 2009, 2012), and endometriosis (Painter et al., 2011).

The results from a recent study among a natural fertility population of Hutterites by Kosova et al. (2010) examining male fertility traits and isolating 41 SNPs, likewise shows promising areas to examine further. In this study, nine of the SNPs were associated with reduced sperm quality, providing a further link to potential biological pathways in men. Due to a strict religious doctrine in the Hutterite community, the variation in non-genetic factors is minimized between individuals, allowing them to confirm the presence of a significant genetic component in the natural variation of fertility. The use of these types of results, particularly in interaction with the socio-environment, could lead to a new understanding of fertility as we know it within the social sciences.

\subsubsection{Polygenic scores (PS)}

The genetic architecture of many human traits appears to be influence by many genes and is therefore polygenic (Visscher et al., 2012). Previous experiences in social science genetics have lead to the conclusion that a small number of SNP cannot explain a large proportion of variance in an outcome, but that: "A typical human behavioural trait is associated with very many genetic variants, each of which accounts for a very small percentage of the behavioral variability" (Chabris, Lee, Cesarini, Benjamin, \& Laibson, 2015, p. 305). As mentioned before, existing evidence on the genetic architecture of reproductive traits such as age at menarche and age at menopause also suggest an underlying polygenic structure. Scholars have proposed creating an additive polygenic score (PS) depending an p-value thresholds from GWAS findings and potentially weighted by effect sizes (Purcell et al., 2009). 
felix-tropf.com

Such scores had considerable success in predicting complex and quantitative outcomes such as schizophrenia (Purcell et al., 2009) or body mass index (BMI) (Speliotes, Willer, \& Berndt, 2010).

Utilizing findings from GWAS studies, such techniques have at least three great advantages for genetically informed social science fertility research. First, if the predictive power of a PS for fertility is sufficiently large, the PS could be used to control for genetic endowment in social science studies, which otherwise might represent unobserved heterogeneity and bias the estimates of other social science predictors in a statistical model (Rietveld, Medland, et al., 2013). Second, with the integration of PS in social science research it becomes possible to approach the biological and social pathways of genes on fertility behaviour by, for example, predicting fertility from education PSs or other related social science outcomes (Beauchamp, 2016; Conley et al., 2016) or test for mediation of fertility PSs in multivariate regression models. Third, it may give new insights into causal pathways amongst social science outcomes. A recent study on the relationship between parental age and schizophrenia, Mehta et al. (2016) show that the wellestablished association between parental age and children's well-being might be partly genetic in nature. So far, the association had been attributed to unstable relationships of young parents and their low economic resources as well as the decreasing (physical) health of older parents which, for example, may complicate conception, pregnancy and birth (Goisis, 2013). Mehta et al. find a U-shaped relationship between the PS for schizophrenia and age at first birth, that is, genes for schizophrenia are associated with relatively old $(>40)$ or young $(<20)$ age at first birth. Given that genes influence parents age at first birth as well as their children's mental health via inheritance, the intergenerational association might partly be spurious and not considering genetic effects may bias findings in social science studies.

\subsubsection{Towards truly interdisciplinary work and quality control}

A central challenge to adopting a biodemographic approach in fertility research has been the lack of training and understanding of very different concepts in the different disciplines. Entering a different field has imminent danger, with the 
social sciences already making some fundamental mistakes via naïve candidate gene studies that have produced false positive findings that will likely fail to replicate. This concern was emphasized by Wachter (2008: 1503), who formulated this key challenge as follows: "the need to keep a high standard of quality control, as interdisciplinary researchers step out beyond their original base of expertise." This means not only borrowing the techniques or data from a particular field, but actively engaging with researchers in those fields and following training to ensure high quality standards. But this is an approach that begs for precious time and resources often unavailable for researchers and requires them (their employers and funders) to possess the ability to understand delayed gratification and long-term thinking, since investments into a new discipline may take years to bear fruit. In the current sphere of competitive research and focus on production, many researchers - and particularly young scientists - may be unable or reticent to make these kinds of longterm investments. Interdisciplinary training programmes and centres could be a valuable solution to train the next generation of truly interdisciplinary scholars.

\subsubsection{Towards more complex models of gene $x$ environment $(\mathrm{GxE})$ interaction}

We have shown that the environment can modify genetic influences (chapters $2 \& 4$ ), however, it is highly likely that there are multiple ways in which the socioenvironment can moderate the genetic endowment of individuals, which remain unknown so far. There are two main ways in which genetic dispositions may influence human fertility. First, there can be a direct effect on physiological characteristics (e.g., fecundity, age at menarche, age at menopause). Second, biological predispositions may affect the processes of decision-making and life course planning, consciously or subconsciously (Kohler et al., 2006). Genes provide the potential for a trait, but environmental conditions determine whether that potential will be realized. The most promising future research will therefore be that which attempts to go beyond the examination of only direct effects of genetic and socio-environmental factors to explore their combined effect and interaction, which is likely greater than their independent effects. It would be interesting to explore how the socio-environment moderates gene expression. It is imaginable that triggers 
felix-tropf.com

such as stress might activate certain hormones and other bodily functions more prevalent in individuals with a particular genetic endowment which in turn could inhibit their fecundability. We also know that social compensation, in the form of social capital and strong networks can result in individuals being able to realize their initial fertility intentions (Balbo \& Mills, 2011). PS, again, are a promising way to test for gene-environment interaction in statistical models.

\subsubsection{Epigenetics}

The growing interest and study of epigenetics is another relevant consideration for future research (Landecker \& Panofsky 2013). This relates to the dynamic nature of genes and how their expression is influenced by DNA methylation. It is plausible that socio-environmental exposures might actually alter gene expression through changes in DNA methylation. For example, the study by Cameron et al. (2005) demonstrated that the maternal behaviour of rats (grooming, nursing) affected gene expression among offspring in the brain regions that control defensive and reproductive behaviours. Another recent study on rats also showed epigenetic silencing or the inhibition of DNA methylation as a mechanism underlying the neuroendocrine control of female puberty (Lomniczi et al. 2013). In humans, factors such as breast-feeding and parental interaction could be potentially interesting factors to study.

\subsubsection{Sex differences}

Almost identical results in heritability estimates for men and women as in Rodgers \& Kohler (2001) might suggest that the same genes are important for male and female fertility. However, the study of Nisén et al. (2013) shows that genes predicting childlessness in women are associated with low education among women and high education among men. From a social sciences perspective, this is not implausible. We know a number of causal mechanisms which explain the association between educational level and fertility and may have different effects for both sexes (Balbo et al., 2013; Mills et al., 2011; Ní Bhrolcháin \& Beaujouan, 2012; 
Nisén \& Myrskylä, 2014), for example opportunity costs of the transition to parenthood and wage penalty (Amuedo-Dorantes \& Kimmel, 2005; Happel, Hill, \& Low, 1984; Liefbroer \& Corijn, 1999). Also biologically, human reproduction is complementary and therefore the genetic architecture of fertility might differ considerably between the sexes (Ellegren \& Parsch, 2007).

In the recent past, several methods have been developed to approach this question for the first time. The GREML models we apply in this dissertation can be used to investigate the genetic correlation in fertility across sexes. Based on GWAS summary results, a new technique has been developed called LD score regression which is able to estimate the genetic correlation across traits and also across sexes on the same trait if the GWAS is conducted separately for women and men (BulikSullivan et al., 2015). Both approaches have recently been used to investigate genetic difference across sexes for height and BMI, demonstrating strong genetic homogeneity for men and women. However, this remains to be investigated for fertility, which appears to be rather complementary: sex-biased and antagonistic gene-expression have been widely discussed and hypothesized in the literature (for review see e. g. Parsch \& Ellegren, 2013).

\subsubsection{A population paradox}

Evolutionary predictions that there is a tendency to have children at a younger age over time seem to contradict widespread observations of fertility postponement at the population level in many European countries. Models from biologists suggest that the AFB decreases across generations (Byars et al., 2010; Kirk et al., 2001), while we observed a massive postponement during the second half of the past century (Mills et al., 2011). Potential explanations for this population paradox are, amongst others, gene environment interaction or additive environmental effects such as the educational expansion or the introduction of the pill overriding smaller effects of natural selection. Once more, this highlights that an integrative approach between biological and social science is necessary to answer questions of human fertility and evolution. 
felix-tropf.com

1.4.8 Integrating new data and methods from quantitative genetics and reproductive medicine

As mentioned at the outset of this introduction, a central reason for the lack of biodemographic research in the area of fertility has been a lack of data that combines good social science indicators with data that has biomarker and genetic data in large samples. The recent significant reduction in the costs of collecting, storing and processing this type of data will hopefully result in new sources for the future. Although considerable research has been done, many questions remain open. The fact that heritability is a population parameter and therefore can vary over time and space requires replication of heritability estimates in different societies of interest.

To break new ground in fertility research using genetic information, methodological advances such as the ACE- $\beta$ model has been developed (see chapter 5). This approach combines insights from econometrics and behavioural genetics by integrating the MZ-fixed effects approach as a direct link between phenotypes into the structural equation model. It therefore potentially identifies a causal link between two traits additional to genetic and environmental endowment particularly if a valid instrumental variable can be found (these and related approaches are detailed in Kohler et al., 2011). More importantly, in the realm of molecular genetics, actual genetic (GWAS) data is being collected and statistical tools further developed (Yang et al., 2010; Yang, Lee, Goddard, \& Visscher, 2011). Using actual genetic information, it becomes possible to determine the heritability of one as well as the genetic correlation between two traits without family data - which is often problematic to collect and requires strong assumptions in the modelling (Conley, Rauscher, Dawes, Magnusson, \& Siegal, 2013). This likewise raises the level of flexibility in testing gene environment interactions and sex differences.

Additional techniques have also been developed within quantitative genetics to try to explain more of the variance predicted in behavioural genetics models or in other words to address the 'missing heritability' problem. Several methods combine many SNPs to aggregate the collective effect and achieve a higher level of predictive power such as polygenic scores (Purcell et al. 2009; Mandemakers et al. 2014) or GREML implemented in genome-wide complex trait analysis software (GCTA, see chapters 3 \& 4) (Yang et al. 2010). Finally, other promising applications include using genes as instrumental variables to go beyond establishing association and make 
causal inferences. Endogeneity problems are often difficult to disentangle, with these approaches offering at least a partial solution (for a critical discussion see Conley, 2009; Taylor, Ware, \& Gage, 2015).

A central conclusion from this thesis is that biological and genetic factors are relevant in explaining and predicting fertility traits. The socio-environment, which demographers and social scientists have studied until now, however, still appears to account for the major part of the observed variance. Studying the interplay between genes and the environment, new data sources and integration of new methods will be central to more effectively understanding and predicting fertility trends. Findings, such as the common genetic base of the first attempt to have a child and NEB furthermore suggest that genes not only underly physical traits important for fertility but also psychological ones. In the coming years we anticipate that the field and interest will grow as we become able to isolate which genetic variants are important for fertility, understand their biological functions and how they interact with the socio-environment. 



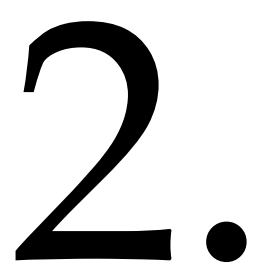

\section{Genetic influence on age at first birth of female twins born in the UK, 1919-68 ${ }^{6}$}

Abstract: Using a sample of monozygotic (945, 42 percent) and dizygotic (1,329, 58 percent) twin pairs born 1919-68 in the UK, we applied innovative tobit models to investigate genetic and environmental influences on age at first birth (AFB). We found that a substantial part (40 percent) of the variation in AFB is caused by latent family characteristics. Genetic dispositions (26 percent) play a more important role than the shared environment of siblings (14 percent), with the nonshared environment/measurement error having the strongest influence (60 percent). Like previous studies, the present one reveals marked changes in estimates over time, and supports the idea that environmental constraints (war or economic crisis) suppress, and normative freedom (sexual revolution) promotes the activation of genetic predispositions that affect fertility. We show that the exclusion of censored information (i.e. on the childless) by previous studies biased their results.

6

This chapter is based on Tropf, F. C., N. Barban, M. C. Mills, M., H. Snieder, \& J. J. Mandemakers (2015). Genetic influence on age at first birth of female twins born in the UK, 1919-68. Population studies, 69(2), 129-145, 
felix-tropf.com

\subsection{Introduction}

There has been a steep increase in age at first birth (AFB) across Europe since 1970. It is now between 28 and 29 years of age (Mills et al., 2011), and is often said to have been subject to a 'postponement transition' (Kohler, Billari, \& Ortega, 2002). The primary reasons proposed for postponement have been women's increased enrolment in higher education and employment in the labour force; but cultural transformations surrounding the timing and role of children have also been proposed as a reason (Balbo et al., 2013; van de Kaa, 1987). Attempts to explain differences and changes in AFB patterns within demography and the social sciences have continued to rely almost exclusively on associations with socio-environmental factors (Goldstein, Sobotka, \& Jasilioniene, 2009; Hobcraft, 2006; Mills \& Tropf, 2016). Yet a growing body of literature has indicated an interplay between individual genetic endowment and the environment, which in turn shapes subsequent lifecourse decisions and fertility behaviour (Freese, 2008; Guo, 2005; Kohler et al., 2006; Mills \& Tropf, 2016; Murphy, 1999; Udry, 1996). Significant genetic influences related to fertility have been established for age at first dating or marriage (Mealey \& Segal, 1993), age at first sexual intercourse (Dunne et al., 1997), number of sexual partners (Guo et al., 2008) and the chance of unprotected sexual intercourse (Daw \& Guo, 2011). One of the strongest genetic effects was found in a twin-study examining the age at first attempt to have a child. The study found that 40-50 percent of the variance was explained by genetic differences (Kohler et al., 1999; Rodgers, Kohler, et al., 2001).

A widely-used technique for estimating the relative influence of genes and environment in explaining AFB is a twin-study. Twin data allow comparisons between monozygotic (MZ), i.e. identical, and dizygotic (DZ), twins and are therefore one of the best resources for evaluating the importance of genetic variation in observed traits (Boomsma et al., 2002). However, existing research that adopted this design to examine AFB has produced very mixed findings. Whereas an Australian study found significant genetic influences (Kirk et al., 2001), research in the U.S. (Neiss et al., 2002) and Denmark (Rodgers et al., 2008) reported nonsignificant effects. The aim of present study was to extend existing knowledge about 
the influence of genetic and environmental factors on AFB in two ways: by replicating previous research on a new population of twins in the UK, and by introducing new analytical techniques that can explain different findings by including or excluding childless individuals. The study addressed three central questions. What are the relative effects of genes and environment in explaining differences in AFB in the UK? Did the pattern of explanation change for cohorts born over the twentieth century? How does the modelling procedure used affect the results obtained?

The current study built upon but also extended previous research in three main ways. First, we introduced more sophisticated statistical models and demonstrated that they produced results different from those of classic designs. The core difference was in the handling of right-censored observations (i.e. on individuals who had not had a first birth when last observed). Reviving awareness of the censoring issue in behavioural genetics (Kohler \& Rodgers, 1999), we examined whether the inclusion of censored information affected heritability estimates of the AFB and if so, to what extent. This was achieved by introducing a tobit structural equation model into the behavioural genetic framework, applying it with censored cases included, and then comparing the findings with estimates obtained using the classic linear structural equation (SEM) models-standard in behavioural genetics-that excluded censored cases.

A second contribution was the application of local linear regressions for each birth cohort in order to describe underlying trends more explicitly (Cleveland, 1979). This allowed us to explore changes over the past century in the influence of latent factors contributing to AFB. Kohler et al. $(1999 ; 2002)$ having demonstrated that the heritability of number of offspring depended strongly on historical and demographic changes during the past century. Our study used the rich information from a large twins register, TwinsUK, in the United Kingdom, which contains information on birth cohorts from 1919 to 1968. It was essential to examine cohort differences because the twentieth-century UK was the setting for distinct social upheavals known to have had a strong impact on AFB (Hobcraft, 1996, 2006; Murphy, 1993).

A third and related substantive contribution was that we studied a new population of UK women. Human traits and behaviour are the result of interplay 
felix-tropf.com

between genetic and environmental factors (Freese, 2008). Genes provide predispositions for complex traits such as AFB, but environmental conditions determine whether such dispositions will be activated. To properly understand gene-environment interactions across societies and time, or in other words the context dependency of genetic effects on fertility, we must evaluate the estimated heritability of a trait in different environments (Guo, 2005). The UK is an interesting case since intergenerational correlations of fertility and AFB are well established and family background has been shown to be a key predictor of fertility over the twentieth century (Booth \& Kee, 2009; Murphy, 1999). It was therefore important to understand the relative influence of genetic and environmental factors on this association.

TwinsUK, the source of our data, is the largest adult twin registry in the UK. It contains data on 12,000 twins and is primarily used to study the genetic and environmental aetiology of age-related complex traits and diseases (Moayyeri et al., 2013). We examined women only, using data available for 2,274 female twin pairs (and thus 4,548 individuals). In the following section we briefly introduce behavioural genetics concepts. We then review previous research on familial and genetic effects on fertility and the course of fertility in the UK over the 20th century. We continue by introducing the TwinsUK dataset and our methods, and follow this with a presentation and discussion of the central findings.

\section{2 Background}

\subsubsection{Intergenerational correlations of fertility}

The motivation for disentagling effects of shared genetic factors from shared family environment on fertility has early roots (for review see chapter 1 or Mills \& Tropf, 2016) and Tropf 2015). Fisher (1930) conducted a study on the number of offspring of more than 2,000 British aristocrats at the end of the nineteenth century and found an intergenerational correlation of 0.20. Murphy (1999) concluded that intergererational correlations of fertility level were very common and had even increased since the end of the nineteenth century. 
Although intergenerational correlations of fertility level seem to follow a consistent pattern, their interpretation remains controversial. One explanation might be that parents each transmit 50 percent of their genetic variants and thus genetic predispositions to their children. With this in mind, Fisher (1930) interpreted the intergenerational correlation of 0.20 as an exclusively genetic effect, which meant that 40 percent of the variance was explained by genetics. Others (Booth \& Kee, 2009) argued that parents also transmit environmental characteristics such as socioeconomic status to their children, which are also important for fertility behaviour. Thus intergenerational correlations between the fertility levels of parents and children lack established interpretations of their genetic and environmental origins, and alternative designs such as twin studies are required to quantify their effects.

\subsubsection{Genetic research on human fertility}

The majority of fertility research that has employed a twin-study design has used data from a historical Danish Twin registry that includes information on virtually every pair of twins born since 1870. Using these data, Kohler et al. (1999; 2002) found evidence of strong genetic influence on number of offspring. They report that the influence was particularly strong following the First and Second Demographic Transitions. The heritability of the number (genetic variance in the number of offspring as a proportion of population variance in the number) was a substantial 0.40 during the strong fertility decline of the First Demographic Transition at the end of the nineteenth century and the Second Demographic Transitions in the second half of the twentieth-century, but was close to zero between the two transitions.

It is the large changes in heritability after the demographic transitions that support the hypothesis of gene-environment interaction. Kohler et al. (1999a; 2002b) associate these changes in genetic influence with the environmental changes that occurred during the transitions. In particular, there were improvements in economic, medical, and hygiene conditions during the First Demographic Transition, while the Second Demographic Transition saw the introduction of oral contraceptives that enabled-and cultural transformations that encouraged-the exercise of personal choice over childbearing (Lesthaeghe, 1995; van de Kaa, 1987). According to the 
standard rationale, the more childbearing (whether and when) is subject to personal choice in a society, the more it is influenced by genetic predispositions (Udry, 1996). The introduction of the contraceptive pill during the Second Demographic Transition is believed to have had a particularly important effect by offering new freedom over the timing of the first child (Lesthaeghe, 1995; van de Kaa, 1987).

The same pattern of gene-environment interaction is suggested by a study of the Danish sample that examined the number of offspring of members of birth cohorts at the age at which 25 percent of them had a first child (Kohler, Rodgers, et al., 2002). This measure of the level of early fertility showed a heritability of 0.52 for cohorts born in the period 1961-68 and therefore socialized after the Second Demographic Transition, when norms encouraging early childbearing had weakened. For the cohorts born in the period 1945-52, this effect was close to zero.

Unlike the fertility studies, investigations of genetic and environmental influences on AFB have shown mixed results. Neiss et al. (2002) conducted a study in the U.S. using constructed kinship data from the National Longitudinal Survey of Youth. Cohorts born in the period 1958-65 were found to have a low heritability of 0.06 for AFB, whereas the shared environment explained up to 20 percent of the observed variance. Similar results were found in Rodgers et al.'s (2008) study of twins from the Danish sample. For birth cohorts for the period 1931-52 (1,242 twins), the shared environment explained 26 percent of the variance in AFB and no genetic effect was found.

In contrast, an Australian twin study reported a significant heritability of AFB. Kirk et al. (2001) investigated the extent of genetic influence common to age at menarche, age at first birth, age at menopause, and number of offspring $(\mathrm{N}=2,710$ twins). The results showed that for cohorts born in the period 1900-65, 21 percent of the observed variance was explained by genetic variance. The shared environment explained 18 percent of the observed variance and the non-shared environmental variance/measurement error was accounting for the largest portion, 61 percent. At the same time, their multivariate biometric models showed that genes influencing AFB were associated with overall reproductive success - measured as the number of children of an individual in relation to the average number of children in a birth cohort - and therefore of the survival of the genetic variants. A recent investigation from Finland (Nisén et al., 2013) of cohorts born in the period 1950-57 shows similar 
results. Genes explained 26 percent of the variance in AFB, shared environment 12 percent, and the non-shared environment/measurement error 61 percent. There was also a genetic correlation between education and AFB meaning that genetic effects on both outcomes have a common base, which partly explains the observed covariation.

Overall, the studies suggest that the influence of genes and the environment on AFB depends on time and place. The treatment of censored information in the estimation of these variables might also play a crucial role. While the studies from the US, Denmark, and Finland included only individuals who experienced a first birth, the Australian models also included childless individuals by imputing the age at last observation, grouping the ages, and estimating an ordered structural equation model (SEM). For this reason, we applied both standard models, which exclude childless individuals, and models that have the ability to capture censored cases. We did this so that we could compare results for the same population.

In what follows, we first briefly describe the fertility context of the UK during the 20th century, and then the TwinsUK register we used. In contrast to previous historical research on genetic effects on reproductive behaviour, our study focused entirely on AFB. However, historically AFB has been strongly related to number of offspring, and changes in this number during the twentieth century have been accompanied by postponement of childbearing (Balbo et al., 2013; Mills et al., 2011). We therefore expected our study to reveal that AFB was subject to patterns of genetic and environmental influences similar to those found for the number of offspring.

\subsubsection{Fertility context of the UK in the $20^{\text {th }}$ century}

Historical analysis on the genetics of fertility focused on the analysis of birth cohorts to reduce environmental heterogeneity among individuals. To interpret the dependence of genetic and environmental contributions to AFB on period, however, we had to examine the historical periods in which the cohorts started childbearing. Figure 2-1 shows the trend in number of offspring and AFB for birth cohorts in the UK between 1925 and 1970. We observe the well established trends: first an increase in the average number of offspring for birth cohorts born during the 1920s and 1930s, 
felix-tropf.com

followed by a steady decline in completed fertility during the second half of the past century. The AFB shows in general the inverse pattern: a decrease in the average AFB for birth cohorts across the 1920s and 1930s, a fall at the beginning of the 1940s, and the striking postponement in the AFB for cohorts born after the end of the Second World War. These trends have been analysed and their direction related to at least five historical periods of social change and upheaval in the UK that have been strongly related to fertility behaviour (see Figure 2-1 for illustration, and Murphy, 1993; Hobcraft, 1996).

In the first of the five periods we considered, British women born in the 1920s grew up and reached maturity during the Second World War, a time of major environmental constraints that affected the timing of AFB: food rationing that began in January 1940; the 'Battle of Britain' in September of the same year; and the call in March 1941 for 100,000 women (but not married women with young children) to 'come into the factories'. Hobcraft (1996) argues that their circumstances made women in these birth cohorts reluctant to start childbearing and they postponed doing so.

In the second period, women born in the 1930s benefited from the new welfare provisions introduced after the war, such as a free National Health Service that included maternity care, family allowances, and free secondary education to 'feed the aspirations of the middle classes' (Hobcraft, 1996, p. 495). Furthermore, the period of the 1950s - when these birth cohorts started childbearing-was a period of changing mores, culminating in the sexual revolution of the 1960s (Hobcraft, 1996). There was a growing insistence on the right of individuals to make their own decisions about sexual conduct and family formation, though the use of reliable contraception was only slowing spreading.

Birth cohorts born in the 1940s and 1950s, the subjects of our third and fourth periods, started childbearing during the 'baby bust' of the 1960s and 1970s, one of the most critical periods for understanding contemporary fertility developments (Hobcraft, 1996). Two crucial factors influenced fertility in these periods. One was the introduction of effective contraception together with the legalization of abortion laws. According to Hobcraft (1996) more than half the fertility decline in the period can probably be attributed to the reduction in the number of unwanted children. 


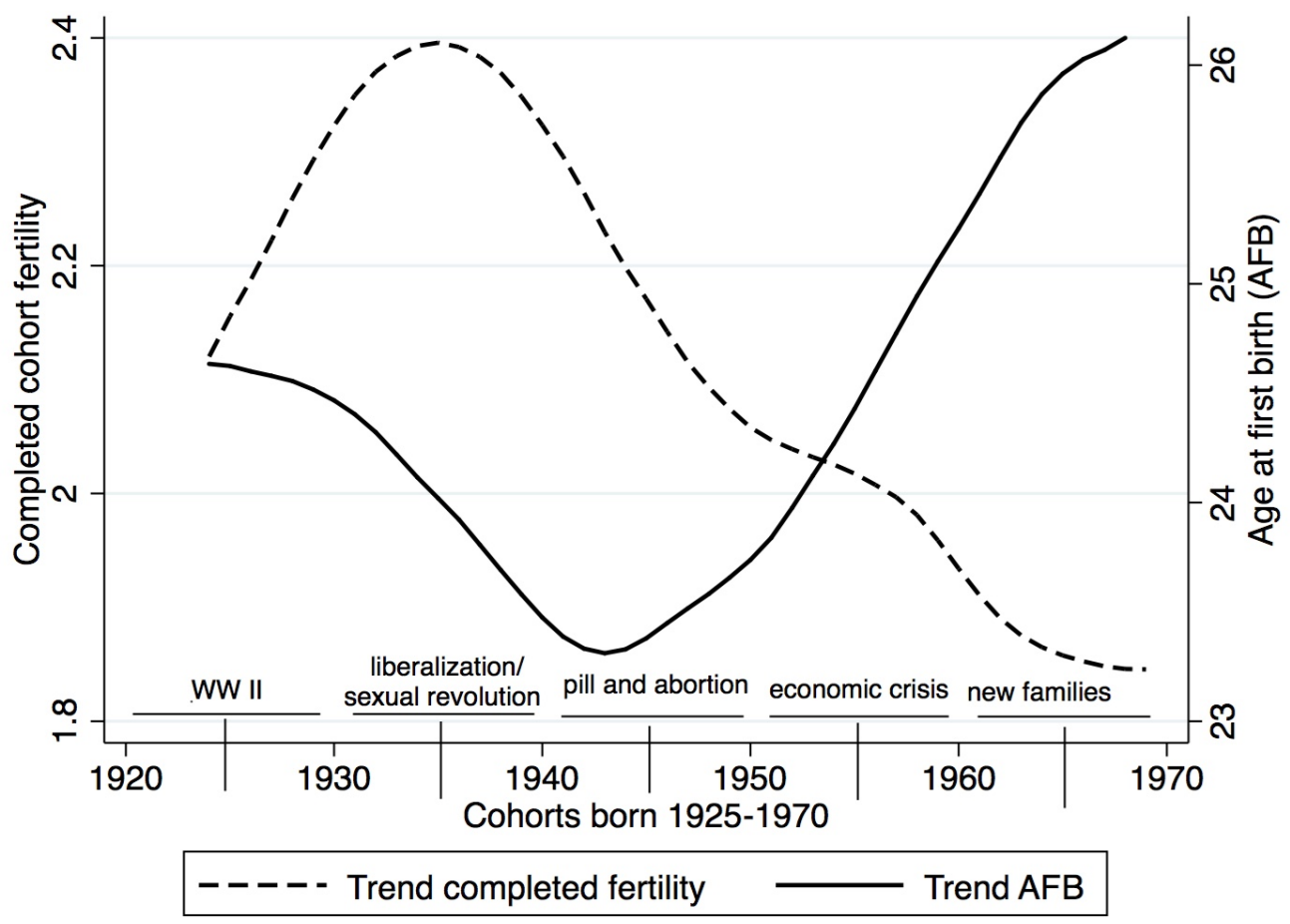

Figure 2- 1 Smoothened scatterplot of the completed cohort fertility and age at first birth in England and Wales (1925-1970)

Source: AFB UK: The estimated average AFB in the UK. Because official data on birth order have been historically only collected within marriage, these values are based on estimates from the Office for National Statistics, Cohort fertility, Table 2 (Office National Statistics, 2013).

The other important influence on the third and fourth periods was the economic crisis of the 1970s. At the end of 1972, wage and rent freezes dominated the UK, and their effect was reinforced by the rise in oil prices in 1973. House prices rose rapidly in 1974 and unemployment passed the one million threshold in 1976, growing to three million in 1982.The proportion of married women who had ever used the pill during the first five years of marriage increased from less than onethird in 1967 to around 80 percent in 1976 (Murphy, 1993). People were able to control childbearing and effectively respond to this period of economic insecurity by postponing childbirth. In fact, England and Wales reached a historical low point in total fertility in 1977 (Murphy, 1993). 
felix-tropf.com

The subjects of the fifth and final period are the birth cohorts born in the 1960s, the members of which entered their twenties during the partnership revolution of the 1980s (Kiernan, 2004). Cohabitation became more socially accepted as a form of partnership and as one able to assume the responsibilities of parenthood. The percentage of children born outside marriage more than doubled during the 1960s, from around 12 percent to around 28 percent.

Given the known dependency on time and prevailing conditions of genetic influences on fertility (Kohler et al., 2006; Udry, 1996), we expected genetic influences to co-vary with environmental upheavals known to influence fertility decisions. We therefore based the interpretation of our findings on their variation across the five historical periods just identified.

\subsection{Data \& method}

\subsubsection{Data}

The subjects of our sample were MZ and DZ twins who voluntarily participated in surveys of the TwinsUK registry. The sample comprises more than 12,000 individuals and has recorded 60,000 observations since 1992 (Moayyeri et al., 2013). The largest adult twin registry in the UK, it has been used primarily to study the genetic and environmental aetiology of age-related complex traits and diseases. The information it collects on participants includes some demographic data.

We aligned the design of our our study with the earlier research, described above, on the early fertility of Danish twins and the number of their offspring. To allow more direct comparisons, we focused on cohorts born before 1968. Data on the AFB were collected by the main questionnaire and, from 2005, also by a behavioural questionnaire.

Respondents to the main questionnaire comprised a sample of 4,989 individuals. Respondents to the behavioural questionnaire added another 1,663 . The combined sample of 6,652 was reduced by the removal of the following: 557 twin pairs because there were missing values in their demographic data; 223 twin pairs because they were males; 238 individuals because their zygosity was missing, 282 because their fertility data were missing, and 24 because they were not Caucasian. 
The removal of these cases resulted in a final sample of 4,548 individuals or 2,274 complete twin pairs. They were members of birth cohorts that ranged from 1919 to 1968, and had been interviewed in the period 1992 to 2010. The average age of the respondents used in the analysis was 57 years (Table 2-1), and most had passed beyond the reproductive period of their lives.

The variables used in our univariate twin models were AFB, age at censoring (C), zygosity, and year of birth. As is standard for survival models (Mills, 2011), age was computed by subtracting the year when a respondent was born from the reported year at first childbirth or the last year of observation in the case of right censoring. For right-censored individuals, we used the age at last observation (ALO) as the censoring age $C_{i}$ and replaced it with age 45 if it was higher. The age of 45 is the commonly assumed end of the reproductive life-span and of the observation window for the fertility of females (Leridon, 2008).

$$
C_{i}=\left\{\begin{array}{r}
A L O_{i} \text { if } A L O_{i} \leq 45 \\
45 \text { if } A L O_{i}>45
\end{array}\right.
$$

Because only one twin had been interviewed in some waves of the survey, we used the life-history information to identify the most recent wave when information for both was available. Zygosity had been established by standard questions about physical similarity and confirmed by multiplex DNA genotyping in cases of uncertainty (Ooki \& Asaka, 2004).

\subsubsection{Bio-social models and the twin design}

The aim of behavioural genetics or bio-social models (Udry, 1996) is to explain observed differences between individuals by differences in genetic and environmental factors. The use of models that require genetic information is rarely considered a requirement for demographic research (Mills \& Tropf, 2016), though the value of multidisciplinary and interdisciplinary studies in social science and demography is becoming more widely recognized (for an overview see Balbo et al., 2013; D'Onofrio \& Lahey, 2010).

The most common way to disentangle the influence of genetic and environmental influences on a trait is to use twin data. Monozygotic (MZ) twins are 
genetically identical (i.e. share all their genotypes) but are assumed to have been exposed to common environmental influences, such as those of family and the neighbourhood in which they grew up. Fraternal or dizygotic twins (DZ), in contrast, are akin to full siblings and share on average 50 percent of their segregating genetic material. They are assumed to share their family environment to the same extent as MZ twins. Thus the degree to which MZ twins are more similar in AFB than are DZ twins reflects the extent of genetic influence on AFB (for details see Boomsma et al., 2002; Snieder et al., 2010).

A naïve way of estimating the proportion of explained variance by additive genetic effects is to compute the correlations of a trait between siblings separately for $\mathrm{MZ}$ and DZ twin pairs. Since they are assumed to share family environment to the same extent, and MZ twins are twice as similar as DZ twins in their genetic endowment, narrow sense heritability $h^{2}$ is twice the difference of the intra-group correlations of MZ and DZ. The effect of the shared environment of the twins is therefore the pairwise correlation of $\mathrm{MZ}$ minus $h^{2}$. Variance that is unexplained by these factors is due to non-shared environmental effects from outside or even within the family (Pike \& Kretschmer, 2009) and measurement error (for details see Snieder et al., 2010). The fitting of genetic models based on this logic has become standard in twin research.

At least three assumptions of the standard behavioural genetics model need to be briefly addressed. The first is that monozygotic and dizygotic twins share their environment to the same extent (the equal-environment assumption (EEA)). This assumption has repeatedly been criticized (e. g. Horwitz, Videon, Schmitz, \& Davis, 2003), though evidence of it not being made is rare in studies (Conley, Rauscher, Dawes, Magnusson, \& Siegal, 2013), including fertility studies (Felson, 2014). Second, it is assumed that there is no assortative mating within the population with respect to the outcome of interest. When the outcome of interest is fertility, this assumption might be considered rather strong, but if assortative mating does obtain, the result is an underestimate of the heritability of genetic influences. The third assumption is that there are no non-additive genetic effects (see also i). Previous studies found slight indications of dominance in their analyses (Kohler et al., 1999) as well as other gene-gene interactions (epistasis) for the physical ability to conceive a child (Christensen, Kohler, \& Basso, 2003). To check for the presence of a 
significant dominant effect, we applied a standard behaviour genetics model-fitting approach to all of our models. The models estimating dominant effects always provided a substantially lower fit with the data (details available on request).

\subsubsection{Structural equation models}

We fitted structural equation models (SEMs) to estimate the influence of genetic and environmental factors on AFB. The underlying logic was in line with the comparison of intra-group correlations of twin pairs described above. However, these correlations have low power and large standard errors and do not make use of information available in variances and covariances. SEM furthermore provides goodness-of-fit statistics thereby enabling us to test and compare alternative models (Snieder et al., 2010).

Figure 2-2 shows the ACE-path model. The capital letters in circles stand for the latent factors assumed to contribute to the observed variance in the sample. Onedirectional arrows refer to the directional non-standardized estimates of the respective variance components of the outcome: 'a' represents additive genetic effects resulting from the sum of genetic effects of alleles (an allele is a variant of a gene for which different variants are possible) from all contributing loci (a loci is a location of a gene on a chromosome); ' $c$ ' are environmental effects resulting from environmental influences shared between twins of a pair; and ' $\mathrm{e}$ ' are non-shared environmental effects resulting from the unique environment of an individual (including measurement error; for details see Snieder et al., 2010). The model was constrained to be identical for each twin at the level of all parameters.

Bi-directional arrows indicate the assumed correlations between the latent variables for both groups of twins, which were introduced earlier. The unique environment ' $\mathrm{E}$ ' is assumed to be independent for both types of twins. The boxes contain the measured outcome variable $Y_{j}$ of the member $j \in\{1,2\}$ of the twin pairs. 
felix-tropf.com

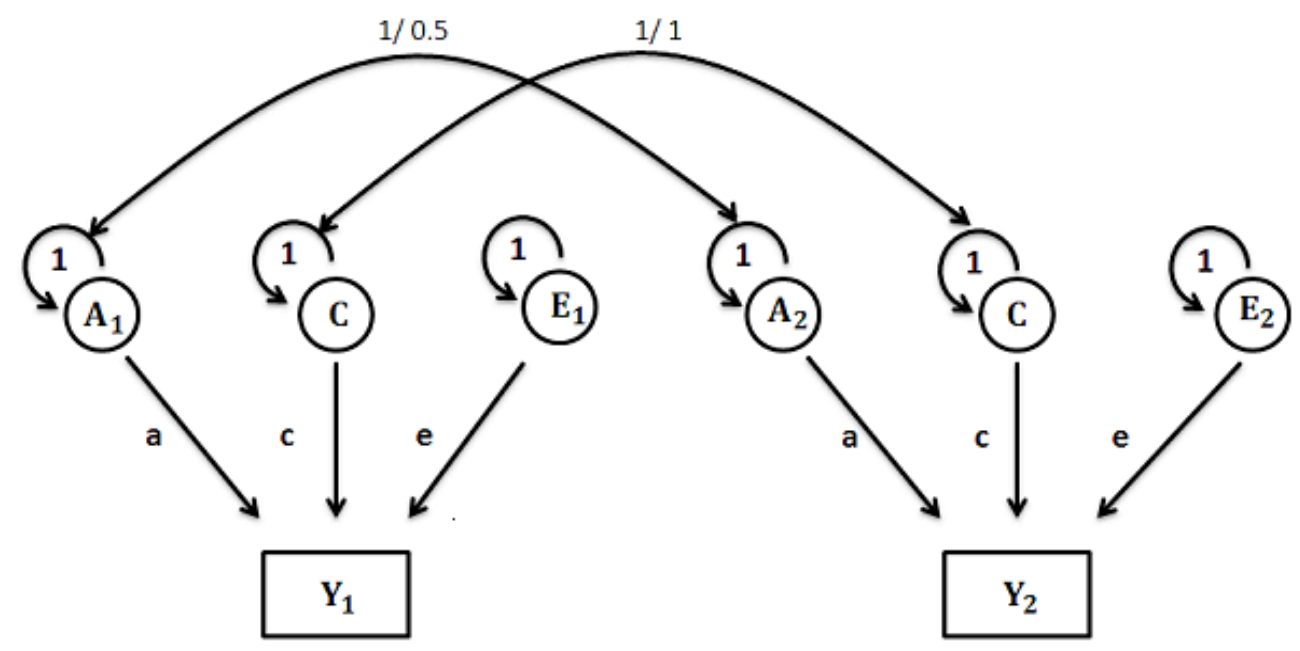

Figure 2-2 ACE path model for the classic twin study (Monozygotic/Dizygotic Twins)

Notes: $A_{1} / A_{2}=$ Genetic Endowment; $C$ Shared Environment; $E_{1} / E_{2}=$ Unique Environment; a $=$ Genetic Effects; c = Shared-Environmental Effects; e = Non-Shared Environmental Effects / Measurement Error; $Y_{1} / Y_{2}=$ Outcome of Twin 1 and Twin 2

The basic structural equations in the model are:

$$
\begin{aligned}
& Y_{1}=a *\left(A_{1}\right)+c *(C)+e *\left(E_{1}\right) \\
& Y_{2}=a *\left(A_{2}\right)+c *(C)+e *\left(E_{2}\right)
\end{aligned}
$$

whereas,

$$
\operatorname{Cov}\left(A_{1}, A_{2}\right)=\left\{\begin{array}{c}
0.5 \text { for } D Z \text { twin pairs } \\
1 \text { for } M Z \text { twin pairs }
\end{array}\right.
$$

and, 


$$
\operatorname{Cov}\left(E_{1}, E_{2}\right)=0
$$

To test how estimates differ between different kinds of censoring treatments, we estimated two models. First, we applied the classic SEM:

$$
Y_{j}=A F B_{j}
$$

where twin pairs with censored information do not contribute to the estimation. This has been the predominant or classic way of modelling these estimates in the literature (Neiss et al., 2002; Rodgers et al., 2008). However, as is known from alternative regression designs, "[b]inary and censored variables can lead to erroneous inferences about heritability in family studies (...)" (Kohler \& Rodgers, 1999, p. 221). Another consequence is the reduction of the sample size. Also this censoring treatment leaves it unclear whether the heritability patterns extend to twin pairs with censored information, which is an issue of particular interest given that the proportion of childless women in the UK doubled from 9 percent for cohorts born in 1946 to 18 percent for those born in 1958 (Kneale \& Joshi, 2008). Projections suggest that of the women born in 1970, from around 18 percent (Bray, 2008) to 22 percent (Sobotka, 2004) will still be childless at the age of 45 . Childless women, therefore, are a group of growing demographic significance (Rendall \& Smallwood, 2003).

In a second step we introduced a tobit model using the R-package twinlm, recently developed to estimate standard univariate twin models with default options and based on an $\mathrm{R}$ package for specifying and estimating linear latent variable models (Holst \& Budtz-Jørgensen, 2012)). These are similar to other prominent openaccess programs such as OpenMx (Boker et al., 2011), but twinlm in the mets package allows the implementation of various statistical models for multivariate event history data such as the tobit model.

In the tobit model, the $A F B_{j}^{*}$ of an individual $i$ is assumed to be a latent, normally distributed trait which cannot be observed over its entire range. If a woman had been interviewed before having a child, we did not know at what point in her future she would have one, whereas the end of the reproductive life-span at age 45 is the end of the observation window for all individuals. We therefore observed: 
felix-tropf.com

$$
Y_{j}=\left\{\begin{array}{c}
A F B_{j}^{*} \text { if } A F B_{j}^{*} \leq C_{j} \\
C_{j} \text { if } A F B_{j}^{*}>C_{j}
\end{array}\right.
$$

The maximum likelihood estimation for censored cases was based on the probability of being censored given the observed values (see also Kohler et al. 1999b). To standardize the estimates and report heritability and shared and non-shared environmental effects/measurement error, we divided $a^{2}, c^{2}$, and $e^{2}$ by the overall variance of AFB and denote genetic effects $h^{2}$. The variance components in the twin model were constrained to be between 0 and 1 , as were the confidence intervals. To test for the significance of the genetic factor, we conducted a likelihood-ratio test on the nested models of the ACE by constraining the genetic component to be zero, which is standard in behavioural genetics. The resulting $p$-values refer to the nullhypothesis that there is no significant loss in model fit if we assume genetic effects to be zero.

\subsubsection{Historical cohort analysis}

For the comparison of cohorts historically, we followed two strategies. The aim of the first was to describe the underlying trend of changes in the genetic and environmental influences on AFB continuously. We therefore applied the tobit ACEmodel to twins from each birth year separately. For these estimates, we used locally weighted scatterplot smoothing (lowess, Cleveland, 1979) for the 10 closest birth cohorts, which allowed us to estimate a focal value for each birth cohort. Local regression estimates were plotted in a smoothed scatterplot of a non-parametric regression of year born on the regression estimates to draw the most complete picture of historical changes in genetic and environmental influence on AFB (for details see Supporting Information 2-1).

Second, we allocated all individuals in the sample to the decades in which they were born. The oldest subsample contained the birth cohorts born in the period 1919-29, and the youngest those born in the period 1960-68. We applied both the classic and the tobit models to all subsamples in order to compare estimates. For the 
local linear regression we also added the confidence intervals for the subsample to the smoothed plot of the latent trend.

\subsection{Results}

\subsubsection{Descriptive findings}

Table 2-1 summarizes the variables for the overall sample $(n=2,274)$ as well as for MZ $(n=945)$ and DZ $(n=1,329)$ groups separately. The mean AFB of the overall sample is 25.6 years with a standard deviation of 4.7 . It is slightly ( 0.4 years) lower for $\mathrm{DZ}$ than for $\mathrm{MZ}$ twins. The percentage of individuals who have not experienced AFB is 16.8 percent-of whom 80.5 percent were older than 45-and information is censored for 27.1 percent of twin pairs. The level of childlessness of MZ twins is 2.4 percentage points higher than that of $D Z$ twins and the former are on average around 2 years older than the DZ twins. The mean AFB of the overall sample follows the population trend in AFB of the UK during the past century quite well at a slightly lower level (see Supporting Information 2-2).

Table 2-1 Summary statistics for female UK twins, birth cohorts 1919-68

\begin{tabular}{|c|c|c|c|}
\hline Sample & MZ \& DZ & MZ & $\mathrm{DZ}$ \\
\hline $\mathrm{N}$ of twin pairs & 2,274 & $41.6(945)$ & $58.4(1,329)$ \\
\hline Percent censored individuals & 16.8 & 18.2 & 15.8 \\
\hline Percent censored pairs & 27.1 & 28.1 & 26.4 \\
\hline Mean (SD) age & $57.0(10.5)$ & $58.0(10.6)$ & $56.2(10.3)$ \\
\hline Mean (SD) age at censoring & $55.4(11.1)$ & $55.0(11.1)$ & $55.7(10.8)$ \\
\hline Mean (SD) AFB & $25.6(4.7)$ & $25.8(4.6)$ & $25.4(4.7)$ \\
\hline Correlation AFB (Pearson) & 0.31 & 0.40 & 0.24 \\
\hline
\end{tabular}

Notes: $\mathrm{MZ}=$ Monozygotic; DZ = Dizygotic; AFB = Age at First Birth; SD = Standard Deviation; Mean AFB and Correlation AFB are computed for individuals who experienced AFB.

Source: TwinsUK

Recall that one of our primary research questions asks to what extent individual differences in AFB can be explained by genetic factors. Table 2-1 reports a correlation in AFB of 0.24 for DZ twin pairs-for uncensored pairs only. DZ twins have the same degree of genetic relatedness as parents and children, and thus this value is in line with findings in Murphy's review of family correlations in fertility 
felix-tropf.com

(Murphy, 1999). The classic correlation in AFB of MZ twin pairs exceeds the correlation of DZ twin pairs in each sample of cohorts. This indicates a genetic component in AFB.

\subsubsection{Twin models}

Table 2-2 presents the results of the ACE model for the two SEMspecifications: the tobit model, which adjusts for censoring, and the classic SEM model. Both models report significant genetic effects on the AFB of females in the UK born in the period 1919-68. Further, these effects exceed those of the shared environment and therefore are the main cause of within-family correlations in AFB. The tobit model yields an estimated heritability in AFB of 0.26. In other words, 26 percent of the observed variance in AFB is explained by genetic variance. Variance in the shared environment of the twins (between-family variance) explains 14 percent of the observed variance. The largest source of variance is attributable to the nonshared environment/ measurement error, which accounts for 60 percent of the observed variance in AFB.

Figure 2-3 shows the scatterplot of the smoothed non-parametric regression curves for the fitted values of the local linear regressions. This exploratory analysis of the data reveals a strong peak in genetic influence for cohorts born at the end of the 1930s. At the same time, the influence of the non-shared environment/measurement error experiences a large drop. It furthermore depicts the continued growth of genetic influence for cohorts born from the end of the 1950s onwards. Whereas the influence of the shared environment is relatively stable, the nonshared environment/measurement error appears to decrease steadily, while it consistently remains the most important cause of variation.

In the most recent cohorts (1961-68), we observe a new pattern with a moderate level of heritability (0.32), and, compared with the previous cohort, an almost constant level of shared-environmental influence (0.26). The non-shared environment/measurement error explains the highest share of the variation in all models, but is the lowest in the most recent cohorts, in which both the shared environment and the genetic influence have a moderate level of influence. 
Figure 2-4 examines the heritablity trend in more detail. It adds confidence intervals based on ACE models for the separate decades of birth cohorts shown in 23 , and shows how the trend in each successive period is related to a significant event in the period. It can be seen that heritability estimates for cohorts born during the second World War are not significant, as is confirmed by likelihood ratio tests provided in 2-3. The rise and peak in heritability are attained by cohorts born during the 1930s, which started childbearing in a period of liberalization and the sexual revolution. This trend reverses for cohorts born during the 1940s, when contraception and legal abortion were introduced, and for cohorts born in the 1950s, who grew up during the economic crises. For these cohorts, heritability estimates once again become non-significant. Finally, there is a steady rise in heritability for women who became parents at the end of the 1950s, a period that saw a relaxation of normative pressure on familial behavior and an increase in the popularity of cohabitation.

Please note that the standardized estimates provided are ratios of the three variance components divided by the total variance, and therefore the estimates shown can also vary with the overall variances. In view of this, we also applied the local linear regressions to the non-standardized genetic component. This showed that our results were robust to change in the overall variances (see Supplemenary Information 2-3).

Table 2-2 shows the ACE-model estimates for both the classic and the tobit models for all decades seperately, illustrating the noticeable differences between the estimates of the two model specifications. In the overall sample, the results for the standard model overestimate heritability by 9 percentage points. Comparing the results across cohorts, we see that there are even notable differences between cohorts of comparable sample size, such as the cohorts of the 1940s and 1950s. The estimates are also not consistent in their direction. While the classic model overestimates heritability of AFB in the first four cohorts, the estimate for the youngest one is lower than the estimate yielded by the tobit model and is non-significant.

Table 2-3 also provides the sample sizes of the sub-models, showing first for the tobit-model that they are particularly small for the oldest (MZ-pairs $=40$, DZpairs $=43)$ and youngest cohorts $(M Z$-pairs $=138$, DZ-pairs=191), and second that 


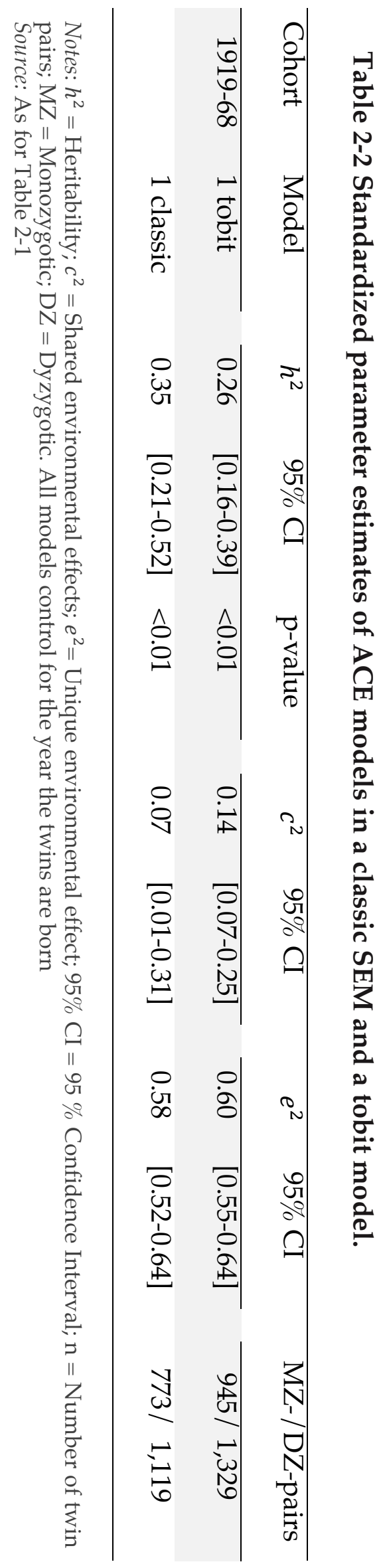


they vary between model specifications. The implication of the small sample sizes is mainly that the non-significant heritability for the oldest cohorts might partly be the effect of power issues. Cohorts born in the 1950s, however, show non-significant results despite relatively larger sample sizes. In order to examine the model differences and the effect of the exclusion of censored twin pairs, we estimated all models in a classic, linear SEM on $C_{i}$ (see Supporting Information 2-4). Results are in line with the tobit model with slightly larger confidence intervals, suggesting that the imputation of the last age of observation remains a robust solution.

\subsection{Summary and discussion}

Our study investigated the relatively understudied topic of the influence of genes and the latent family environment on the AFB, and specifically on their influence on cohorts of UK twins born in the period 1919-68. The findings indicate that a substantial part-40 percent-of the variation in AFB is caused by latent family influences, 26 percent by genetic factors, and 14 percent by the shared environment of siblings. The strongest influence-60 percent-is that of the nonshared environment/measurement error, an influence to which the characteristics of the partner are likely to make a major contribution (Kohler, Rodgers, et al., 2002). These findings suggest that genetic effects on reproductive behaviour are too strong to be ignored in demographic and sociological research on fertility.

There are two main ways in which genetic effects may influence human fertility. First, there can be a direct effect on such physiological characteristics as fecundity. For example, the age at which a female experiences menarche is strongly influenced by genetic variants (Anderson, Duffy, Martin, \& Visscher, 2007). This is also the age at which a woman is able to become pregnant. Thus, depending on the extent to which genes directly influence AFB, social scientists who do not take genetic effects into account actually underestimate the contributions of their predictors to the explanation of socially driven variance in reproductive behaviour (Kohler \& Rodgers, 2003) 


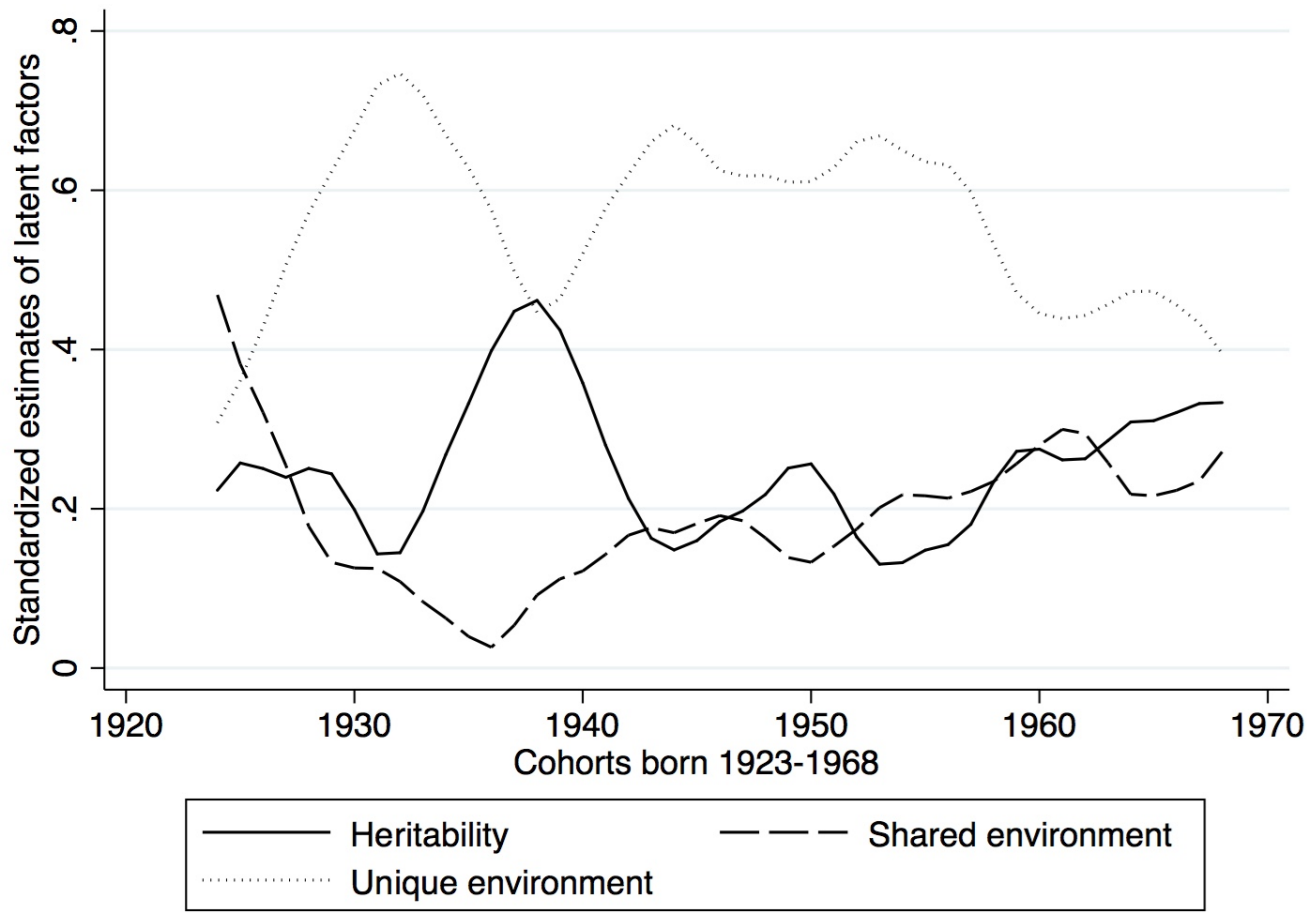

Figure 2-3 Smoothened scatterplot of local polynomial regressions of the birth year on the latent factors estimates for birth cohorts 1923-1968

Notes: Smoothened results of local linear regressions based on the 10 closest birth cohorts. Cohorts Born Between 1923-1968: For birth cohorts

Source: Same as for Table 2-1

Second, genetic influences may affect the processes of decision-making and life-course planning both consciously and subconsciously (Kohler et al., 2006). In fact, biologists now suspect that behavioural and psychological traits that are subject to genetic influences are those most closely linked to fertility, or more generally, to reproductive fitness (Kirk et al., 2001). Evidence of the importance of genetic influences on behaviour includes evidence of their effect on the age at first attempt to get pregnant (Kohler et al., 1999; Rodgers, Kohler, et al., 2001). This suggests that part of the substantial genetic influence on the AFB is mediated through psychological traits. 


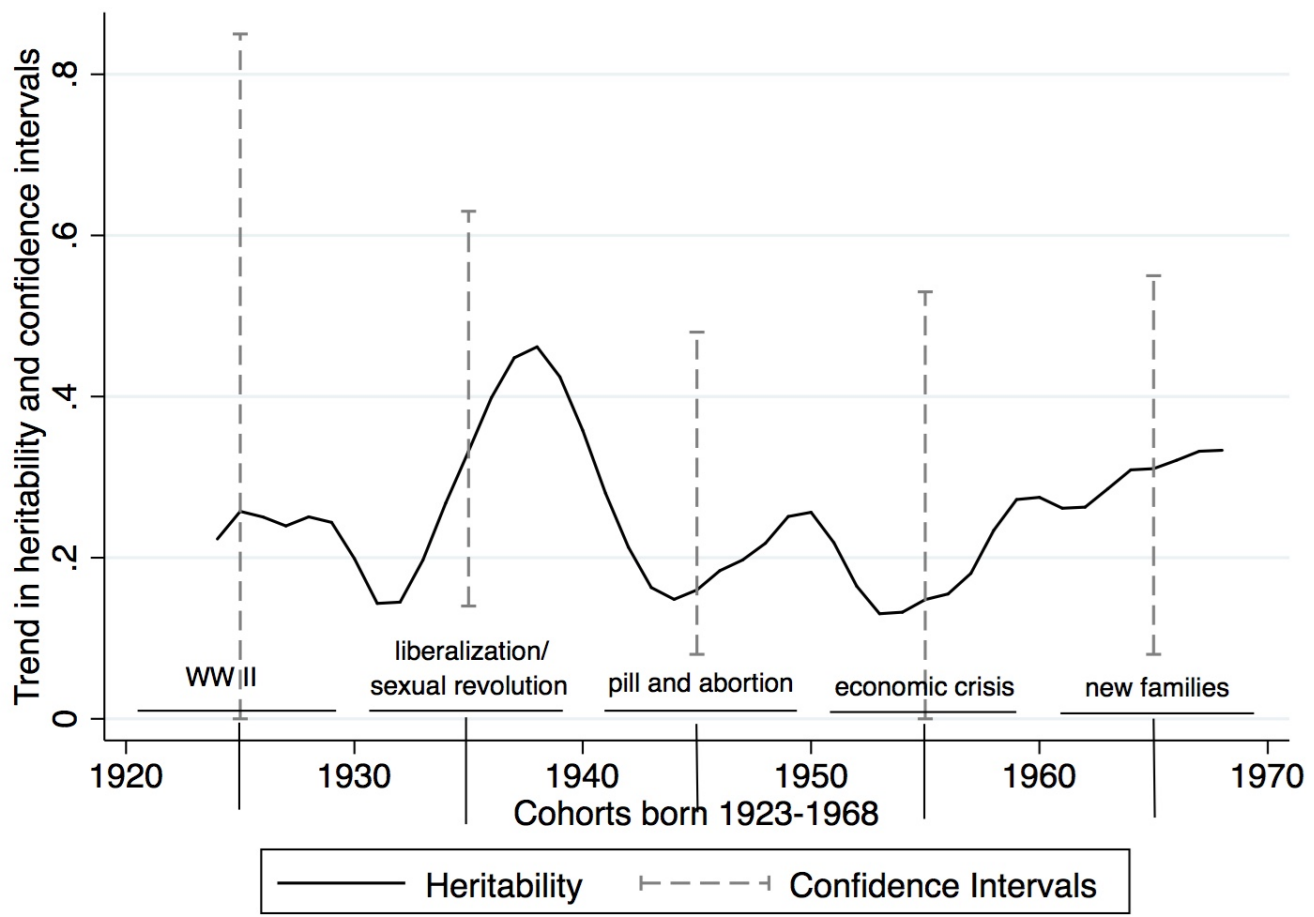

Figure 2-4. Smoothened scatterplot of local polynomial regressions of the birth year on heritability estimates for birth cohorts 1923-1968 and the historical context

Notes: Smoothened results of local linear regressions based on the 10 closest birth cohorts. Confidence intervals for decades are based on estimates in Table 2-3. Cohorts Born Between 1923-1968.

Source: Same as for Table 2-1

Fertility behaviour is often seen to depend on the extent to which factors affecting the life course are experienced as certain or uncertain (Mills, Blossfeld, \& Klijzing, 2005; Oppenheimer, 1994). The state of the social environment also appears to affect the extent to which biological predispositions affect the processes of decision-making (Rodgers, Bard, \& Miller, 2007; Udry, 1996). Thus research that has revealed large changes in the extent of genetic influences on fertility suggests that the main reason for the changes has been variation in the extent to which people have felt free to choose whether and when to become a parent and in what familial circumstances. In our study, economic uncertainty appears to override and freedom from normative constraints to encourage the activation of genetic effects on decisions about childbearing. In particular, economic crises or exogenous shocks, such as those posed by war, are environmental conditions that may override 
individual differences in genetic influences on attitudes to childbearing. In contrast, freedom of choice in family formation, sexual behaviour, and birth control in modern societies are believed to encourage the activation of genetic influences on individual fertility behaviour. That is why, as a second step in our study, we examined trends in the latent factor estimates for birth cohorts born in the period 1919-68. This revealed the pattern predicted by the foregoing considerations.

For women who entered adulthood during the Second World War, when major environmental constraints forced postponement of childbearing, we found no significant genetic influence on the AFB. In contrast, for individuals born in the 1930s, who benefited from liberalization of cultural values and changing sexual norms, heritability estimates show a strong peak. This pattern reverses for cohorts born in the 1940s, who started using effective contraception (the pill), as well as legal abortion. According to Hobcraft (1996), the fall in period fertility between 1964 and 1977 can probably be attributed largely to a reduction in unwanted births, but also to normative constraints on births outside marriage. For the first time, women were enabled by contraception to control their own fertility and avoid childbearing when it was normatively inappropriate, thus making it possible for environmental influences to override and suppress genetic influences on fertility. And in fact their influence becomes non-significant for cohorts born in the 1950s, which faced severe economic disruptions during the 1970s and the beginning of the 1980s-a period of economic crisis in which there was a historical low point in total fertility (Hobcraft, 1996).

For cohorts born in the 1960s, we observe a new pattern: an increase in genetic influence but with a still relatively moderate level of family influencemirroring a trend revealed in an earlier study of genetic influences on fertility behaviour (Kohler et al. 1999). These cohorts experience a new freedom in family and career planning (Van de Kaa, 1987; Lesthaeghe, 1995). Rodgers et al. (2008) maintain that the influence of stable environmental conditions after the Second Demographic Transition has had an ongoing effect on women's career planning and education, in ways that have competed with their fertility planning. 


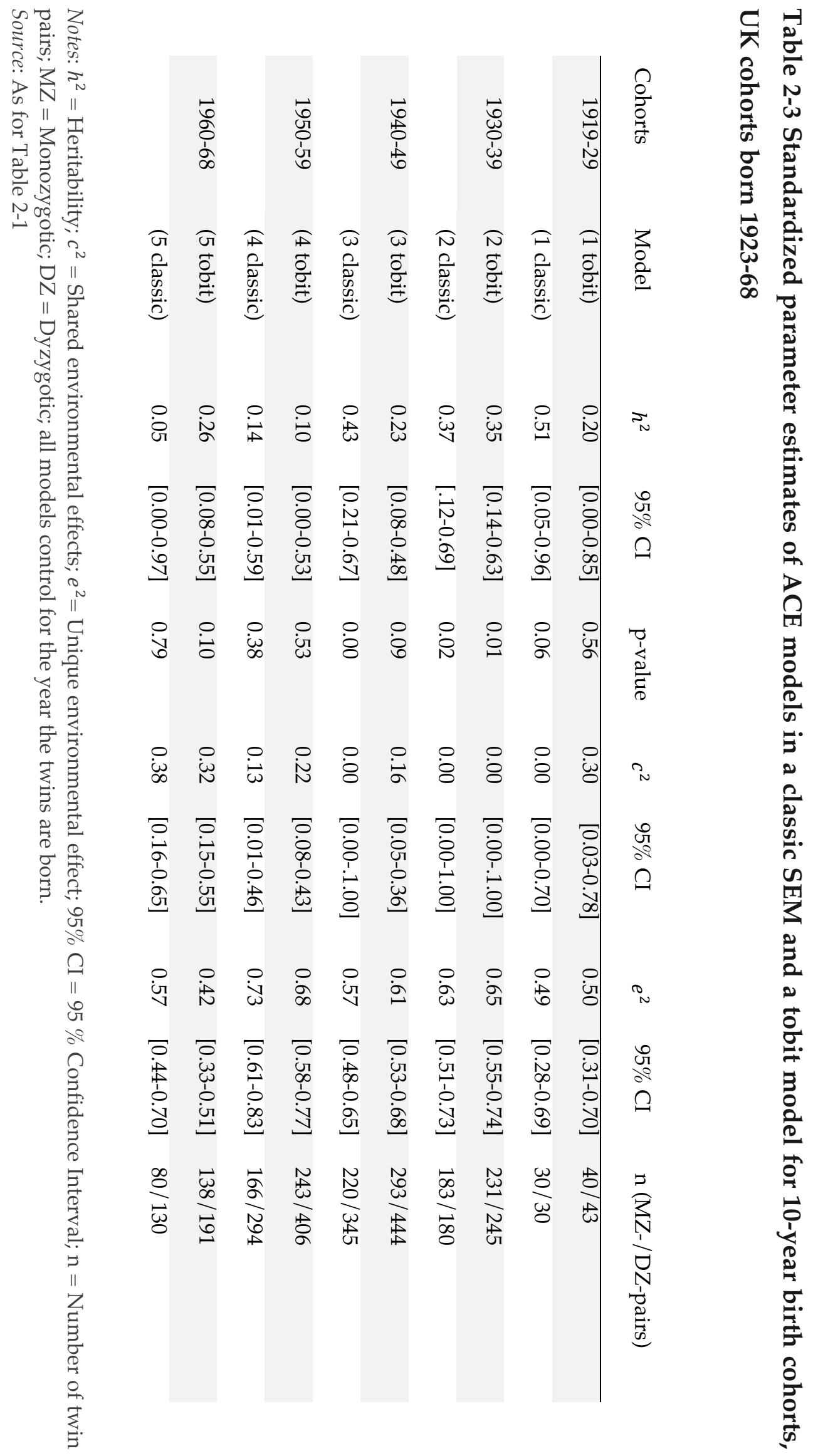


felix-tropf.com

Overall, our findings emphasize the importance of the study of geneenvironment interaction and its relationship with fertility behaviour. The results are consistent with the findings of Kohler et al. (1999) for Denmark, which showed a peak in heritability for completed fertility in the second half of the past century. The pattern we found thus appears to be robust for completed fertility and AFB in Denmark and the UK. A recent study by Bras et al. (2013) also supports our interpretation of the findings of these studies. That study replicated the increase in heritability during the first Demographic Transition, using a database containing details of 100,000 sibling pairs born in the period 1810-70 in the Dutch Province of Zeeland. Similarly a study by Rodgers et al. (2007), using data from the National Longitudinal Survey of Youth (NLSY), shows that different genes are important throughout the life course, emphasizing the need for a life-course approach in behavioural genetics.

Although our theoretical reasoning provides a basis for the interpretation of our results, we did not provide a statistical test of gene-environment interaction. The differences in heritability estimates are not significant across birth cohorts and therefore remain suggestive. Replication is required, as are improvements in method. We believe that ongoing genome-wide data collection and complementary developments in methods of analysis (Tropf, Stulp, et al., 2015 see also chapter 3; Yang, Lee, et al., 2011; Yang et al., 2010) will provide new insights and opportunities to address questions of gene-environment interaction directly and at the micro level.

Previous research studying the genetics of AFB has produced mixed results. While Kirk et al. (2001) reported findings broadly in line with our estimates from the tobit model, research from the US (Neiss et al. 2002: Rodgers et al. 2008) and Denmark found no significant genetic effect. We argued that this might be because these studies also used different analytical strategies. Kirk et al. (2001) applied an ordered probit model with individuals censored at age of last observation and age groups categorized to follow a normally distributed latent variable. Neiss et al. (2002) excluded childless observations (21 percent) and Rodgers et al. (2008) included only twin pairs in which both siblings had experienced a first birth, and applied a classic linear SEM.

To demonstrate how different analytical strategies affect the outcomes, we applied two kinds of model: a recently developed tobit model in the SEM framework 
and the classic SEM that excludes censored cases for the covariance estimation. We found large differences between the two approaches, with different directions and degrees of deviation. We concluded that the choice of analytical strategy is critical. In particular, the exclusion of censored information appears to bias the estimate. We also applied classic models that imputed censored cases and found that they produced virtually the same results as the tobit model.

It is essential to note that fertility outcomes differ not only vary depending on the modelling strategy adopted, but also with the demographic and genetic characteristics included. Including only individuals who experience a first birth in the models gives a precise measure of AFB by focusing on a subset of the population that has had at least one child. But there are costs: important information in the data is not used, and the generalization of the results is restricted to a subpopulation. Epidemiological research that has investigated genetic influences on other complex traits, such as blood pressure, supports the idea that subjects with extreme but unknown values of an outcome or phenotype (e.g. the use of antihypertensives) should not simply be excluded, because this leads to a bias in the components of familial variance and loss of power to identify genes (Cui, Hopper, \& Harrap, 2003; Tobin, Sheehan, Scurrah, \& Burton, 2005). When comparing populations that differ in their extreme values, such as birth cohorts with different levels of childlessness, it is particularly important to include all individuals, in order to have comparable samples.

Our findings have important implications for future research. First, the two measures of AFB are genuinely different. As the outcomes for both demonstrate, there is no clear directional pattern in the results, and therefore no general conclusions can be drawn on how the inclusion or exclusion of childless individuals might affect results. For reasons given above, we believe that future studies should utilise a measure that includes childless individuals.

Second, censoring alone cannot entirely explain the mixed findings in the literature. First, in contrast to the studies by Neiss et al. (2002) and Rodgers et al. (2008), in our study the exclusion of childless individuals does not only lead to lower, but in also to higher heritability estimates in subsamples. Second, the study by Nisén et al. (2013), which also excluded childless couples, reports a moderate level of heritability in Finland. We conclude that, while our study demonstrates how results 
felix-tropf.com

can depend on the treatment of censored cases within one population, it is probable that gene-environment interaction plays an additional role in explaining the mixed findings across populations.

In conclusion, we draw attention to two substantive issues. First, it has been suggested that genes not only interact with social and psychological factors, but are mediated them (Rodgers et al. 2001). We believe future research should extend efforts to find effective genetic variants, and to understand their function in relation to fertility behaviour. Second, the association between AFB and completed fertility needs to be studied in more detail. Kirk et al. (2001) found a robust genetic covariation between AFB and completed fertility for Australian twins: genes predicting early AFB are associated with a higher number of offspring. Tropf et al. (2015) confirm these findings using molecular genetic data on samples from the Netherlands and the UK. As Milot and colleagues (2011) conclude in a study of a population of natural fertility, AFB decreased within the past 300 years as a response to natural selection. In our view, multivariate genetic models need to be applied on large samples to determine the degree of genetic covariance in AFB and completed fertility and to better understand and predict the timing of human fertility. 


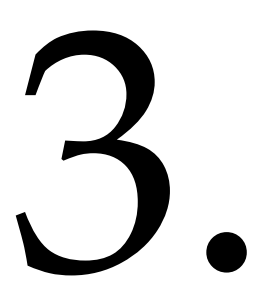

\section{Human fertility, molecular genetics, and natural selection in modern societies ${ }^{7}$}

Abstract: Research on genetic influences on human fertility outcomes such as number of children ever born (NEB) or the age at first childbirth (AFB) has been solely based on twin and family-designs that suffer from problematic assumptions and practical limitations. The present study exploits recent advances in the field of molecular genetics by applying genomic-relationship-matrix based restricted maximum likelihood (GREML) methods to quantify, for the first time, the extent to which common genetic variants influence the NEB and the AFB of women. Using data from the UK and the Netherlands $(\mathrm{N}=6,758)$, we find significant additive genetic effects on both traits, explaining 10 percent $(\mathrm{SE}=5)$ of the variance in the NEB and 14 percent $(\mathrm{SE}=4)$ in the AFB. We further find a significant negative genetic correlation between AFB and NEB in the pooled sample of -0.62 ( $\mathrm{SE}=0.27, \mathrm{p}$-value $=0.02)$. This finding implies that individuals with genetic predispositions for an earlier AFB had a reproductive advantage and that natural selection operated not only in historical, but also in contemporary populations. The observed postponement in the AFB across the past century in Europe contrasts with these findings, suggesting evolutionary effects were overridden by environmental ones, and underscoring that evolutionary predictions in modern human societies cannot be straight forwardly tested. It emphasizes how an integrative research design from the fields of genetics and social sciences is needed to understand and predict fertility outcomes. Finally, our results suggest that we may be able to find genetic variants associated with human fertility when conducting GWAS-meta analyses with a sufficient sample size.

7 This chapter is based on Tropf, F. C., G. Stulp, N. Barban, P. M., J. Yang, H. Snieder, M. C. Mills (2015). Human Fertility, Molecular Genetics and Natural Selection in Modern Societies. PloS one, 10(6), $\mathrm{e} 0126821$. 


\section{1. Introduction}

Recent research within both biology (Byars et al., 2010; Kirk et al., 2001; Milot et al., 2011) and demography (Kohler et al., 2006; Mills \& Tropf, 2016) points to a genetic component of human fertility, namely the number of children ever born (NEB) and the age at first birth (AFB) of women, explaining up to 40-50 percent of the observed, respectively phenotypic variance in these traits. The well-established negative relationship of late AFB with lower NEB (Mills et al., 2011; Sobotka, 2004) appears to be partly genetic, suggesting that natural selection favored a younger age at first birth over the Twentieth century (Byars et al., 2010; Kirk et al., 2001; Milot et al., 2011). Genetic studies examining the relationship between NEB and AFB, however, have been solely based on twin (Kirk et al., 2001) or other family designs (Byars et al., 2010; Milot et al., 2011) that use data on expected genetic differences among relatives to estimate the genetic component underlying these traits. Although these studies are common in behavioural genetics, they can only draw indirect inferences about genetic contributions and suffer from problematic assumptions and practical limitations (critical discussions on, for example, the equal environment assumption (EEA) can be found in ref(Devlin, Daniels, \& Roeder, 1997; Hettema, Neale, \& Kendler, 1995; Horwitz et al., 2003). The studies are limited for further reasons. First, by virtue of their design, twin studies inherently require pairs of siblings and therefore exclude individuals from low fertility families, particularly only children, which may be problematic for the generalization of results. Second, dizygotic twinning has in contrast to monozygotic twinning a genetic base (Hoekstra et al., 2008; Mbarek et al., 2016; Montgomery, Zondervan, \& Nyholt, 2014), which means that dizygotic twins potentially carry genes important for high fertility. Therefore, the use of monozygotic and dizygotic twins to investigate fertility questions in the classic twin design leads to a non-random genetic stratification and might bias variance estimates. Finally, a practical limitation of family designs is that they require data from multiple family-members, which are obviously more difficult to gather than data on unrelated individuals.

An ideal design to examine the genetics of fertility would be a direct estimate using single nucleotide polymorphisms (SNPs) across the entire genome for unrelated individuals who do not share the same micro environment, which was 
first applied to height as a model complex trait (Visscher et al., 2010; Yang et al., 2010). The current study exploits recent advances in the field of molecular and quantitative genetics by applying genomic-relationship-matrix restricted maximum likelihood (GREML) methods to quantify for the first time the extent to which common genetic variants influence both the NEB and the AFB of women. We applied both uni- and bivariate models to these traits, producing unbiased estimates of their common SNP heritability and the extent to which the association between earlier AFB and higher lifetime fertility (NEB) is due to a (negative) genetic correlation between AFB and NEB (S. H. Lee et al., 2012). This not only helps us to understand the relationship between the AFB and NEB, but also allows an assessment of whether genes are associated with a reproductive advantage, indicating natural selection in contemporary, industrialized populations.

In contrast to twin and family designs, the GREML approach is free of confounding from shared environmental effects between close relatives because the method can be applied in a sample of unrelated individuals (Visscher et al., 2010; Yang et al., 2010). GREML analyses make use of the genetic similarity between pairwise unrelated individuals as captured by all common SNPs and correlate the genetic similarity with the phenotypic similarity between individuals (see material and methods). To ensure accurate and well-powered estimates, particularly for the bivariate model (Visscher et al., 2014), we pooled data sources to estimate the genetic influence on all outcomes of interest (see material and methods). We utilize two large cohorts, one from the Netherlands ( $N L, N=4,338$ ) and one from the United Kingdom (UK, $\mathrm{N}=2,420$, for descriptive statistics see Table 3-1). In both populations, resemblance in fertility outcomes has been reported for relatives (Murphy, 1999; Rijken \& Liefbroer, 2009; Steenhof \& Liefbroer, 2008) using intergenerational comparisons with survey data. However, no distinction between genetic and environmental effects responsible for this pattern could be made so far. After quality control of the merged genetic data files, we used more than 1 million SNPs to estimate the genetic relationships among the individuals (see material and methods) and subsequently the genetic variance components.

The most successful and popular design to detect the approximate location of genetic variants associated with a complex trait is the meta-analyses of genome-wide association studies (GWAS) from multiple samples. In lieu of this, our assessment of 
felix-tropf.com

the genetic effects of common SNPs based on the pooled samples shape the expectations to find individual variants when conducting a GWAS. We account for population stratification effects by adjusting for the first 20 principal components in our GREML models. Population stratification refers to allele frequency differences due to systematic ancestry differences. Population stratification can cause spurious associations if not adjusted properly (Price et al., 2006). We furthermore correct for country and birth cohort effects as well as dizygotic twinning. From the twin data only singletons are included, so that close relatives do not contribute to the estimates.

This study has several important implications for research in demography, genetics and biology. We know surprisingly little about genetic effects on human fertility at a population level, yet it is crucial for our understanding of fertility, the interpretation of related social science research in this field (Benjamin et al., 2012; Kohler \& Rodgers, 2003; Rietveld, Cesarini, et al., 2013; Rietveld, Medland, et al., 2013; Rodgers et al., 2008) and broader questions of modern human evolution (Byars et al., 2010; Milot et al., 2011; Stearns et al., 2010; Zietsch et al., 2014). We first discuss the importance of adopting an integrative multidisciplinary approach to understand human fertility before proceeding with an introduction of the methods and the presentation and discussion of our findings.

\section{2 Towards an integrative approach in human fertility} research

The term 'fertility' takes on different meanings in demography, reproductive medicine and biology (Mills et al., 2011). In demography, fertility refers to performance, specifically the two interrelated aspects of the tempo of childbearing (in our case age at first childbirth, AFB) and the quantum or number of children ever born (NEB) in a certain period (J Bongaarts \& Feeney, 2000). In reproductive medicine, fertility defines the ability/inability of couples to conceive and have children given unprotected intercourse (Joffe, 2010). In biology, AFB and NEB have become central indicators for individual fitness as the successful transmission of genes to the next generation in post-industrial societies (Byars et al., 2010; Stearns et al., 2010), with particularly NEB shown to be nearly perfectly correlation with 
alternative measures (Goodman, Koupil, \& Lawson, 2012; Kirk et al., 2001). Due to improvements in hygiene and the reduction in prenatal, infant and child mortality in industrialized societies, NEB has emerged as the standard to measure lifetime reproductive success-that is, biological fitness (Stearns et al., 2010).

In the last decades, industrialized societies have experienced massive changes in both the postponement of AFB and drop in the total number of offspring, which cannot mainly be attributed to genetic or biological factors (Balbo et al., 2013; Mills et al., 2011). Rather, human reproduction is influenced by three analytically distinct but empirically interrelated factors: 1) genetic and biological fecundity (i.e., length of reproductive period, infertility diseases), 2) the environment (i.e., institutional and family structures); and, 3) reproductive choice of individuals (i.e., planned behavior, latent individual and partner characteristics).

Previous research has successfully demonstrated that there is a genetic component to reproduction with over 70 genome-wide association studies (GWAS) published for 32 traits and diseases associated with reproduction (Montgomery et al., 2014). This includes identification of genes such as those related to age at menarche (Liu et al., 2009; Sulem et al., 2009), menopause (He et al., 2009; Perry et al., 2009; Stolk et al., 2009, 2012) and endometriosis (Painter et al., 2011). Environmental factors, such as women's gains in education and labor market participation, gender equity and economic uncertainty have been shown to strongly impact the tempo and quantum of fertility (for reviews see Balbo et al., 2013; Mills et al., 2011). Studies of reproductive choice, which have examined the predictive power of fertility intentions on behaviour, often position reproductive choice in a sociopsychological framework that consists of attitudes (perceived costs and benefits), norms (influence social network) and perception of control over individual choice (Ajzen, 1991; W B Miller \& Pasta, 1994).

A bivariate twin model in a study by Rodgers and colleagues (Rodgers, Hughes, et al., 2001) suggests an interrelation between reproductive choice and genetic factors, providing evidence for shared genetic effects on the decision to have a first child and the number of children during one's lifetime. It is therefore likely not only that biological fecundity, the environment and reproductive choice interact with each other, but also that genes influence reproductive choice. Genetic endowment in social science fertility research has been virtually ignored (Balbo et 
al., 2013), yet may be of major importance when interpreting the observed associations.

If the quantum of fertility in the form of NEB is at least partly genetically influenced, this implies that certain SNPs have a higher chance of being successfully transmitted to the next generation than others, and by extension that the allele frequency might change due to natural selection-that is, that evolution may be occurring. If the negative relationship between AFB and NEB is partly genetic, this would indicate that the AFB was under natural selection during the Twentieth century and that more recent birth cohorts may carry a higher genetic predisposition for an earlier AFB.

Using a family-design, findings from the Framingham Heart Study demonstrated that the same genes influencing NEB are negatively correlated with the AFB (Stearns et al., 2010). The authors subsequently predict that selective changes in the disposition for the timing of the first child predict the decrease in the AFB for subsequent generations. The study design, however, is based on correlations between relatives and the estimates can therefore be inflated by shared environmental factors such as family norms that are important for fertility (van de Kaa, 1987). Family designs cannot robustly discriminate between the case where the correlation between NEB and AFB is environmentally caused, and natural selection, where the correlation is genetically caused and the allele frequencies of the genome might change (Stearns et al., 2010). This limitation leaves a less desirable practical solution: "...to note the issue and remain modest in drawing conclusions" (Stearns et al., 2010, p. 613). In the current study, our design does make it possible to directly draw conclusions about contemporary natural selection based on information derived from the field of molecular genetics. When the trait of interest, here the age at first birth, does not genetically covary with fertility, a genetic response to selection will not occur (Morrissey, Kruuk, \& Wilson, 2010).

\section{3 Data \& method}

\section{3. 1 Data}

For the Netherlands, we use data from LifeLines, a multi-disciplinary prospective population-based cohort study examining, in a unique three-generation 
design, the health and health-related behaviours of 167,729 persons living in the North of The Netherlands, including genotype information from more than 13,000 unrelated individuals (Klijs et al., 2015). It employs a broad range of investigative procedures in assessing the biomedical, socio-demographic, behavioural, physical and psychological factors which contribute to the health and disease of the general population, with a special focus on multi-morbidity and complex genetics.

For the UK, we use data from TwinsUK, the largest adult twin registry in the country with more than 12,000 respondents (Moayyeri et al., 2013). Due to our analytical strategy, we randomly selected only one twin for analysis and controlled for dizygotic twinning as a genetically related process. We recognize that for generalizability a population-based sample such as LifeLines is more desirable for the models we present. The descriptive statistics of the phenotypic variables in the genotyped subsamples with full fertility information are shown in Table 3-1.

\section{3. 2 Genotypes}

Since genotyping had been performed using different chips in the UK and the Netherlands, we use imputed data to aid the alignment of both datasets. The HapMap3 imputation panel has been shown to be reliable for GREML analysis (S. H. Lee, Wray, Goddard, \& Visscher, 2011).

We received genotype data from TwinsUK and Lifelines, which we imputed according to the 1000 genome panel, after which we selected HapMap3 SNPs with an imputation score larger than 0.6. For quality control (QC), we excluded the SNPs with a larger missing rate than 1 percent, lower minor allele frequency than 1 percent and which failed the Hardy-Weinberg equilibrium test at a threshold of $10^{-6}$ for both datasets. We merged the TwinsUK and the Lifelines samples and quality controlled the merged dataset in the same way again. On average 1,017,420 SNPs could be utilized to estimate the GRM between individuals. We used the software Plink (Purcell et al., 2007) for the quality control and merging of the two datasets. 
felix-tropf.com

\section{3. 3 Phenotypes}

\section{Number of children ever born}

Number of children ever born measures the number of children a woman has given birth to including stillbirths. This has been asked directly in the twinsUK ("How many children have you given birth to?") or we constructed it using questions about the year of childbirth of each child. In Lifelines, respondents have been asked to list the birth and death date of children from their current and previous partner with up to 6 children in both categories. For the Lifelines and part of the TwinsUK questionnaires, information for the date of death of the children was given. In both datasets less than 0.2 percent of the children had not reached reproductive age and the correlation of number of children ever born and number of children reaching reproductive age was $>0.98$.

Since fertility is strongly age dependent, we focus on women with completed fertility history in reference to the phenotype. In general, the end of the woman's reproductive lifespan occurs around the age of 45 (Leridon, 2008). Thus, we only included women aged 45 or older in our analysis of NEB. Furthermore, in vitro fertilization (IVF) - often related to twinning and multiple births - can bias results if IVF compensates for genetically based infertility. However, in our TwinsUK sample, only 60 women reported using IVF, and these were excluded in the final analyses. Age at first birth

To calculate the AFB, we used information on the year of childbirth of the first child and year of birth of the mother. In TwinsUK, information from an additional behavioral questionnaire directly asking for the age at first birth in 2005 was available. Childless individuals were treated as missing in the analysis.

\section{3. 4. Heritability estimates}

The genetic component underlying a trait is commonly quantified in terms of heritability $\left(h^{2}\right)$ as the proportion of the genetically caused variance $\left(\sigma_{G}^{2}\right)$ over the overall phenotypic variance of the trait (phenotype, $\left.V_{P}\right)$ ): 


$$
h^{2}=\frac{\left(\sigma_{G}^{2}\right)}{\left(\sigma_{P}^{2}\right)}
$$

Whereas the phenotypic variance is the sum of genetic and environmental $\sigma_{e}^{2}$ variance components.

$$
\sigma_{P}^{2}=\sigma_{G}^{2}+\sigma_{e}^{2}
$$

The methods we applied have been detailed elsewhere (Visscher et al., 2014, 2010; Yang, Lee, et al., 2011; Yang et al., 2010). Briefly, we applied a mixed linear model

$$
y=g+e
$$

where $y$ is an $\mathrm{Nx} 1$ is vector of dependent variables, $\mathrm{N}$ is the sample size, $\mathrm{g}$ is the Nx1 vector with each of its elements being the total genetic effect of all SNPs for an individual, and e is an Nx1 vector of residuals.

We have $\mathrm{g} \sim \quad N\left(0, \sigma_{G}^{2} A\right)$ and $\mathrm{e} \sim N\left(0, \sigma_{e}^{2} I\right)$, where $\sigma_{G}^{2}$ is the genetic variance by all SNPs, $\boldsymbol{A}$ is the genetic relationship matrix (GRM) estimated from SNPs, $\sigma_{e}^{2}$ is the residual variance and $\boldsymbol{I}$ is an identity matrix. The variance components are estimated using the restricted maximum likelihood (REML) approach. The NEB is not normally distributed (see Supporting Information 3-1). This might bias the inference, yet simulation studies show that there is no bias even for binary traits (S. H. Lee et al., 2011). Still, we base our p-values on likelihood-ratio tests, comparing the full model with one constraining genetic effects to be zero (Snijders, 2011).

The estimates of heritability obtained using GREML can be interpreted as the proportion of variance of a trait based on a large set of common genetic variants genotyped. The method is based on the genetic relatedness among individuals measured on about one million of SNPs.

This analysis has been extended to a bivariate approach by Lee and colleagues (2012) to estimate unbiased genetic correlation based on a standard bivariate linear mixed model combined with the genome-wide genetic relatedness matrix.

Genetic correlation 
felix-tropf.com

The genetic correlation $(r(G))$ is an estimate that standardizes the genetic covariance between two traits $\operatorname{Cov}\left(G_{t 1, t 2}\right)$ by the genetic variance of both traits:

$$
r\left(G_{t 1, t 2}\right)=\frac{\operatorname{Cov}\left(G_{t 1, t 2}\right)}{\sqrt{V_{G_{t 1}}} * \sqrt{V_{G_{t 2}}}}
$$

If the genetic correlation between two traits is 1 , all genetic variance in trait 1 and 2 has a common origin. If the genetic correlation is 0 , the genetically based variances of trait 1 and trait 2 are independent.

Phenotypic and genetic correlation analysis

The phenotypic correlation between two traits $r\left(P_{t 1, t 2}\right)$ is the sum of genetic and environmental influences shared across traits and can be estimated like this:

$$
r(P)=\sqrt{h_{t 1}^{2}} * r\left(G_{t 1, t 2}\right) * \sqrt{h_{t 2}^{2}}+\sqrt{e_{t 1}^{2}} * r\left(E_{t 1, t 2}\right) * \sqrt{e_{t 2}^{2}}
$$

whereas $h_{t i}^{2}$ is the heritability of trait $\mathrm{i}$ in the model and $e_{t i}^{2}$ is the environmental or residual variance contribution for the trait, standardized for the overall variance

$$
e^{2}=\frac{\sigma_{e}^{2}}{\sigma_{P}^{2}}=1-h^{2}
$$

And $r\left(E_{t 1, t 2}\right)$ is the environmental or residual correlation between the traits (for the estimates of environmental effects see Supporting Information 3-4). We can solve this to compute the fraction of the phenotypic correlation explained by the genes (or the environment respectively the residuals). For the transformation of standard errors, the delta-method has been applied (Lynch \& Walsh, 1998). 


\section{4 Results}

\subsubsection{Descriptive findings}

Table 3-1 shows the descriptive statistics for both traits in the TwinsUK and the Lifelines cohorts. Overall the AFB is around one year later in the Dutch (26.83) than in the British cohort (25.70), and the British women are about 9 years older. These characteristics are interrelated, since Europe experienced a massive postponement in the AFB during the second half of the Twenties century (Mills et al., 2011), so the larger proportion of younger individuals leads to a later average AFB in the data.

To combine the cohorts, both fertility measures, AFB and NEB, were standardized by country (Z-transformation) and the NEB was log transformed to approach normal distribution (see Supporting Information 3-1 for distributions and 3-2 for the model estimation of all alternative transformations - estimates are robust across transformations).

\subsubsection{The correlation between AFB and NEB}

In line with previous studies, women who had their first child at a later age had a lower number of children ever born (Fig. 1) in both the British and Dutch samples. The observed correlation for individuals with full information on both traits (therefore excluding all childless individuals, individuals younger than 45 and individuals without information about the AFB) between AFB and NEB is -0.32 $(\mathrm{N}=1,521)$ in the UK cohorts, $-0.26(\mathrm{~N}=2,553)$ in the Dutch cohorts and $-0.28(\mathrm{~N}=$ $4,074)$ for the standardized measures in the pooled cohorts $(-0.27$ if estimated from the residuals of all covariates, not listed).

\subsubsection{SNP heritability of AFB and NEB}

Table 3-2 depicts the SNP based heritability $\left(\mathrm{h}_{\mathrm{SNP}}^{2}\right)$ estimated from the univariate models for AFB and NEB. Both traits have a significant genetic component, with $h_{\text {SNP }}^{2}$ for NEB of 0.10 (SE 0.05) and for the AFB of 0.14 (SE 0.04). These results suggest that additive effects of common SNPs explain 10 percent of the variance in the NEB and 14 percent of the variance in the AFB of women. 
felix-tropf.com

\subsubsection{Bivariate GREML analysis of AFB and NEB}

Table 3-3 shows the results of the bivariate GREML model for AFB and NEB, including the genetic correlation between both traits. The genetic correlation would be $-1.00 / 1.00$ if all genetic effects of AFB and NEB are shared, and 0 if the genetic effects of AFB and NEB are completely independent. The genetic correlation estimate is -0.62 (SE 0.27) and significantly different from 0 ( $\mathrm{p}$-value $=0.02$ ), meaning that genes which lead to a later age at AFB are indeed negatively associated with the NEB. Based on these estimates, genetic effects lead to a phenotypic correlation of 0.07 (0.03) between AFB and NEB, whereas the overall correlation estimated from the fitted model is $-0.38(\mathrm{SE}=0.02)$. Therefore around 20 percent of the phenotypic correlation is attributable to shared genetic effects across the traits, while still the main part is associated with common environmental/residual effects of AFB and NEB. The phenotypic correlation estimated from the genetic model is larger than the observed correlation because the bivariate GREML analysis does not require both traits to be measured on exactly the same set of individuals; that is, it makes use of additional information, e.g. childless individual for the estimates of NEB. If we only include individuals with full information on both traits in the genetic model - as we do when computing the phenotypic correlation directly - the phenotypic correlation estimated based on the genetic model $(-0.29 \mathrm{SE}=0.02)$ is not significantly different from the observed value of the Pearson correlation $(-0.27)$, and the component due to genetic effects estimated from the GREML model $(-0.08 \mathrm{SE}=0.05)$ is not significantly different from the estimate using all available information $(-0.07 \mathrm{SE}=0.03)$, whereas the inference would be weaker (see Supporting Information 3-4 for the model excluding all individuals with missing information). 


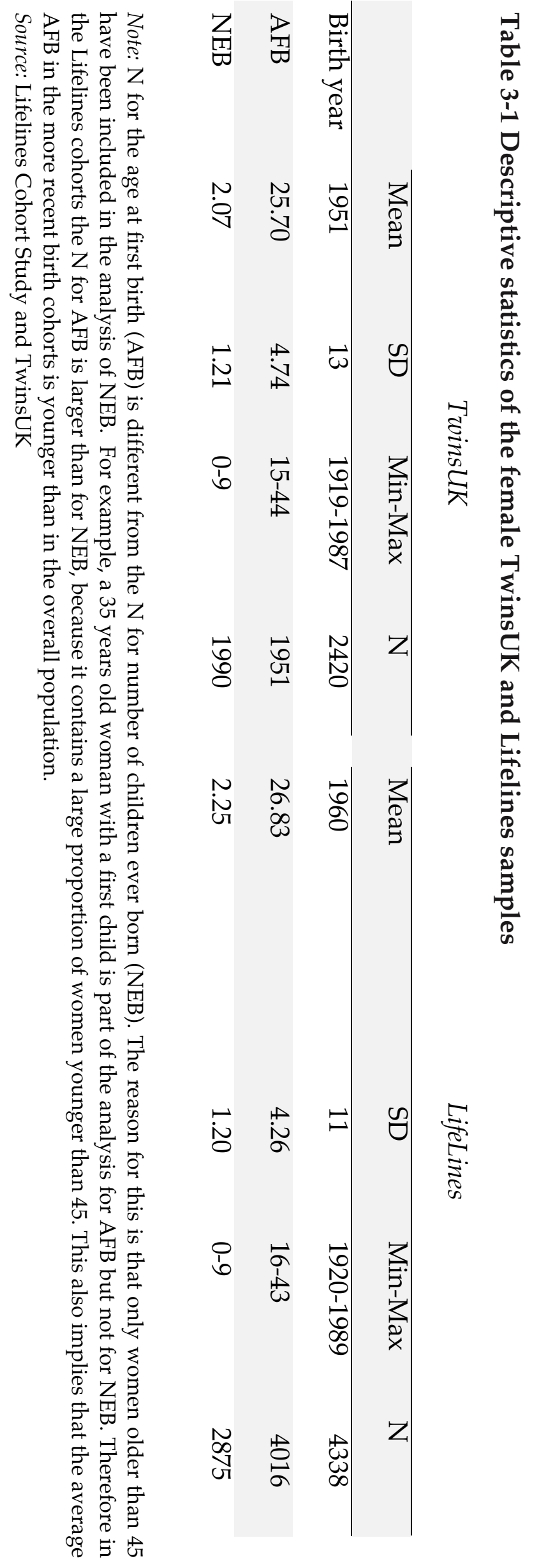


Table 3-2 Heritability estimates of NEB and AFB for the pooled sample of women from the UK and the Netherlands using information from about 1 million SNPs

\begin{tabular}{llll} 
& $\mathrm{h}_{\text {SNP }}^{2}(\mathrm{SE})$ & $p$-value & $\mathrm{N}$ \\
\hline Number of children ever born $^{\mathrm{a}}$ & $0.10(0.05)$ & 0.02 & 4865 \\
Age at first birth $^{\mathrm{b}}$ & $0.15(0.04)$ & 0.0004 & $5967^{\mathrm{d}}$
\end{tabular}

Notes: a: standardized by country and log transformed to adapt the distribution; b: standardized by country; c: p-values are based on likelihood-ratio tests, the reference model constraints genetic effects to be 0; Please find estimates of untransformed variables in SI 3-3; $\mathrm{d}$ : The $\mathrm{N}$ for age at first birth is larger than for number of children ever born. The reason is that only women with completed fertility history are included for the latter (for discussion see material and methods and Table 3-1.).

Source: Same as Table 3-1

\section{5 Discussion}

Using recently developed analytical techniques from molecular genetics we provide direct evidence for a genetic component underlying the AFB and NEB of women in the UK and the NL born during the Twentieth century. Moreover, genetic effects on the tempo (AFB) and quantum (NEB) of human reproduction co-vary, which partly explains why women who start reproducing at an earlier age have higher fertility.

This genetic association between AFB and NEB can have different origins. Both traits might simultaneously be influenced by the same genetic effects (pleiotropy). Alternatively, genetic effects on the NEB could be mediated via the AFB, or some combination of the two. To further examine the causal relations between these factors, actual measured genotypes important for these traits could be integrated into the statistical models (van den Oord \& Snieder, 2002) for applications such as Mendelian randomization (Verduijn, Siegerink, Jager, Zoccali, \& Dekker, 2010) - although this has been challenged (Conley, 2009). Whatever the underlying cause of the genetic association between NEB and AFB, as the consequence of this genetic association we expect natural selection acting in modern, industrialized societies, implying that women born in more recent cohorts may be genetically 
inclined to have an earlier AFB. This prediction of a decrease in AFB, however, is somewhat of a 'population paradox' because it strongly contradicts observed fertility trends over the last 50 years. Instead, there has been a massive postponement in the AFB of an average of 4-5 years in nearly all European countries and the US since the 1970s (Mills et al., 2011).

Although our results seem to generate a paradox, they are well in line with studies on natural fertility populations, such as from Milot and colleagues (2011), who observed a decrease in AFB as a response to natural selection in a contemporary population. One probable explanation is that natural selection works in addition to environmental forces and in the opposite direction, with the latter being far stronger. Natural fertility populations are assumed to have set fertility norms that maximize reproductive success. In the absence of contraption, full reproductive potential can be realised (Milot et al., 2011). In European and many industrialized societies, in contrast, environmental changes across the past century such as the use of contraceptives and women's educational expansion and entry into the labour market have had a strong impact on fertility behaviour (Mills et al., 2011; Sobotka, 2004). This which has led to a postponement in the AFB even though more recent populations in the Netherlands and the UK are genetically predisposed to an earlier AFB. In that sense, the environment has overridden the forces of natural selection.

The discrepancy between observed changes in fertility and those predicted by evolutionary processes show parallels to the case of human height. Although there is evidence for negative natural selection height at least in US populations (Byars et al., 2010; Stulp, Verhulst, Pollet, \& Buunk, 2012), people still, on average, grow up to be taller than their parents (Komlos \& Lauderdale, 2007). This is also largely attributed to environmental factors, such as better nutrition and improved health care (Steckel, 1995). Natural selection, however, may also work in tandem with environmental factors: a recent study suggests that, in the Netherlands, natural selection favoured taller heights, and thus reinforced the effects of improved environmental quality over the last 150 years (Stulp et al., 2015). 

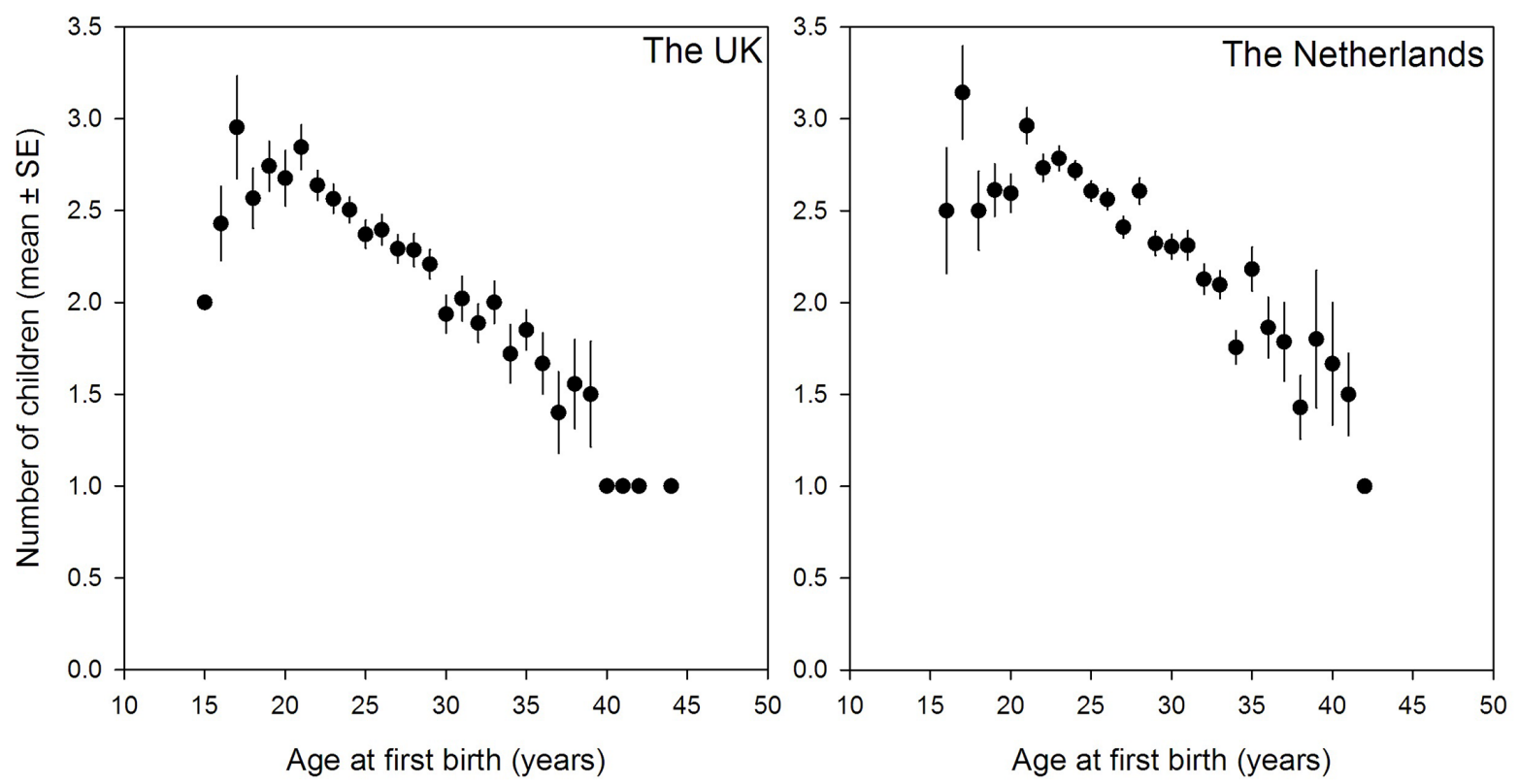

Figure 3-1 The association between age at first birth and number of children ever born in the British and the Dutch cohorts.

\section{Source: Same as Table 3-1}

A second potential - and largely related - explanation for the fact that AFB is postponed despite selection towards genes favouring earlier birth is that genes and the environment interact across birth cohorts. Previous twin studies have in fact shown differences in heritability estimates across cohorts and environments in both NEB (Kohler et al., 1999) and AFB (Kirk et al., 2001; Neiss et al., 2002; Nisén et al., 2013; Rodgers et al., 2008; Tropf, Barban, et al., 2015). Therefore, independent of additive environmental effects leading to postponement in AFB, genetic variants important for the AFB may differ across cohorts and populations, so that large changes due to natural selection do not necessarily occur.

The genetic effects estimated in this study represent narrow sense heritability estimated from SNP data. As would be expected (Vinkhuyzen, Wray, Yang, Goddard, \& Visscher, 2013), they are lower than the estimates of narrow sense heritability ( 0.20-0.30) obtained from family designs. Potential reasons for this are, on one hand, the inflation of estimates by shared environmental factors in family designs, and on the other hand, true genetic effects of variants that are not captured through linkage disequilibrium with SNPs used in GREML analysis. In order to 


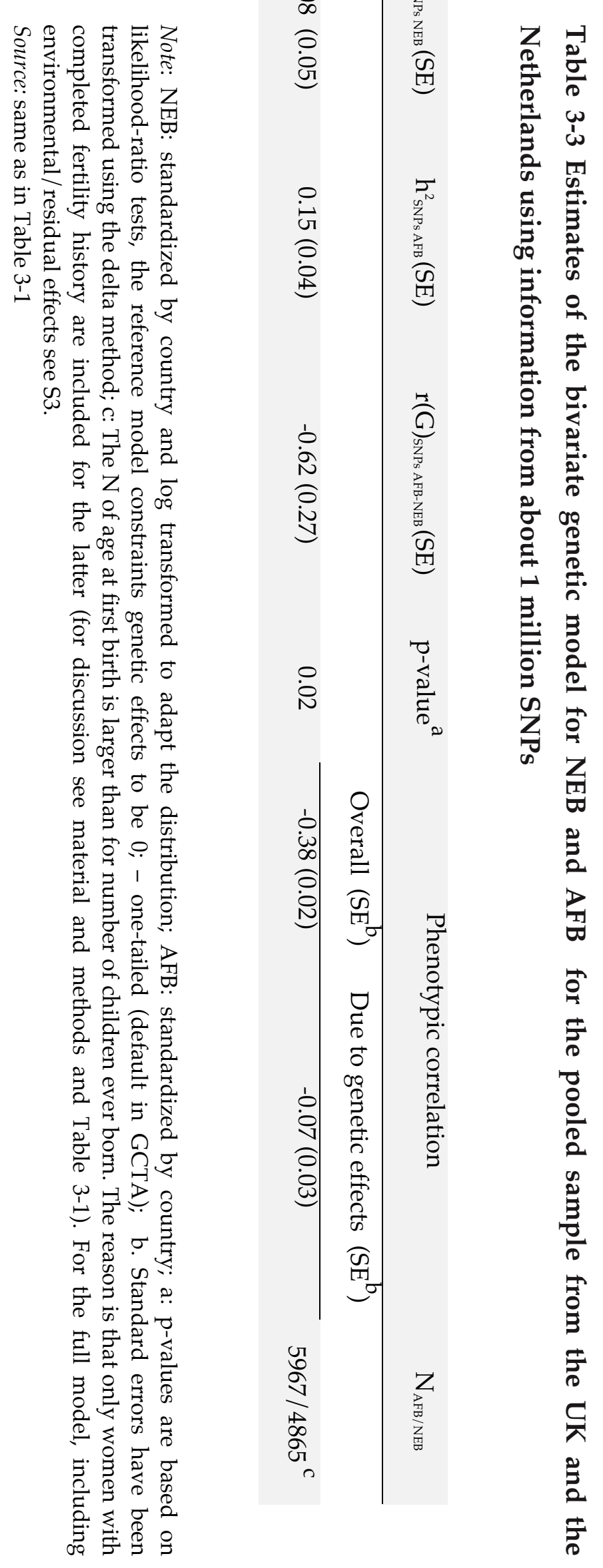


more rigorously examine of genetic effects as well as gene-environment interplay, replication in larger datasets and across populations is required. The provision of data with genetic and environmental information continues to grow, as do more advanced analysis techniques (Zaitlen et al., 2013).

Nonetheless, it becomes obvious that human fertility is both a genuinely biological process as well as a social undertaking. We conclude from our findings that an integrative approach between the social and biological sciences is necessary to better understand the changing patterns in, and even to predict future, levels of human fertility.

Despite the significant advances in the estimation techniques and sample size of this study, there are two limitations that need to be made explicit. First, the interpretation of NEB in an evolutionary manner implies an interpretation of NEB as a measure of fitness. It would be better to have information on the number of children who entered reproductive age or even more appropriate, the number of grandchildren entering reproductive age; this would permit a more precise measure of how far genes have been successfully transmitted across generations. However, NEB has been shown to be a good measure of reproductive success (Stearns et al., 2010) due to diminishing mortality during the reproductive lifespan. Recent genetically informed research furthermore demonstrates that the same which influence NEB also influence the number of grandchildren born and therefore have a long-term effects (Zietsch et al., 2014). Second, in contrast to most research in demography (Mills, 2011), it is still uncommon to address cases right censored information (i.e., those who have not yet had a child by the time of observation) in genetic studies. In our study, we treated individuals who remained childless as missing when estimating genetic influences for the AFB, since they did not yet have a child. Childless individuals, however, are of great interest for demographic research as well as from an evolutionary perspective - since they are the ones who do not transmit their genes to the next generation. While the structural equation modelling in twin studies provides alternative solutions such as Tobit (see chapter 2 and Tropf, Barban, et al., 2015) or ordered models (Kirk et al., 2001) to integrate censored information, there remains no possibility of doing this in current applications of GREML. In general, the observed association can be expected to be stronger when including childless individuals, because all childless women are 
right-censored cases. However, it has been shown in twin models that it is more difficult to predict the effect on the genetic analysis. Therefore, the extent of empirical differences between our model and survival models must be tackled in future research.

To date, thousands of genetic variants have now been successfully linked to physical or psychological traits (Hindorff et al., 2009; Visscher et al., 2012), as well as complex 'socio-genetic' traits like educational attainment (Rietveld, Medland, et al., 2013) and traits related to reproduction (Montgomery et al., 2014). We conclude that our study, based on the same genetic data as in GWAS studies, raises confidence that we will find genetic variants associated with human fertility when conducting GWAS-meta analyses of a sufficient sample size. 



\section{Mega-analysis of 31,396 individuals from 6 countries uncovers strong gene- environment interaction for human fertility ${ }^{8}$}

Abstract: Family and twin studies suggest that up to 50 percent of individual differences in human fertility within a population might be heritable. However, it remains unclear whether the genes associated with fertility outcomes such as number of children ever born (NEB) or age at first birth (AFB) are the same across geographical and historical environments. By not taking into account this possibility, previous genetic studies implicitly assumed that the genetic effects are constant across time and space. We conducted a mega-analysis by applying whole genome methods on 31,396 unrelated men and women from six Western countries. Across all individuals and environments, common single-nucleotide polymorphisms (SNPs) explained only $\sim 4$ percent of the variance in NEB and AFB. For individuals belonging to the same population and demographic cohort (born before or after the $20^{\text {th }}$ century fertility decline), SNP-based heritability was almost five times higher at 22 percent for NEB and 19 percent for AFB. We also found no evidence that genetic effects on fertility are shared across time and space. Our findings imply that the environment strongly modifies genetic effects on the tempo and quantum of fertility, that potentially ongoing natural selection is heterogeneous across environments, and that gene-environment interactions may partly account for missing heritability in fertility. Future research needs to combine genetic research with social science to better understand human fertility.

8

This chapter is based on a manuscript by Tropf, F. C., R. M. Verweij, P J. van der Most, G. Stulp, A. Bakshi, D. A. Briley, M. Robinson, A. Nyman, T. Esko, A. Metspalu, S. E. Medland, N. G. Martin, H. Snieder, S. H. Lee, M. C. Mills. A preprint is published on bioRxiv, doi:http:/ / dx.doi.org/10.1101/049163

A modified version is currently under peer review in an international science journal. 
felix-tropf.com

\subsection{Introduction}

Twin and family studies from Western countries show that genetic factors may explain up to 50 percent of the differences in human fertility outcomes such as number of children ever born (NEB) or age at first birth (AFB) within a population (Byars et al., 2010; Kirk et al., 2001; Kohler et al., 1999, 2006; Mills \& Tropf, 2016; Milot et al., 2011; Rodgers, Kohler, et al., 2001; Tropf, Barban, et al., 2015). It remains unknown, however, whether the same genes are important for fertility across different environments or whether gene-environment interaction modifies genetic effects on fertility. This is an important question for at least three reasons. First, the most successful and widely-used design to detect the approximate location of genetic variants associated with complex traits is a meta-analysis of genome-wide association studies (GWAS) from multiple populations (Visscher et al., 2012). This approach assumes genetic effects on a trait to be universal across environments. However, in the case of fertility, this assumption may not be valid given that environmental upheavals such as the introduction of the pill or educational expansion have substantially changed fertility behavior in the recent past (Balbo et al., 2013; Mills et al., 2011). A second and related point is that heritability studies relying on molecular genetic data result in lower estimates than family studies (Manolio et al., 2009) - and this is true also in fertility research (Kohler et al., 2006; Mills \& Tropf, 2016; Polderman et al., 2015; Tropf, Barban, et al., 2015; Zaitlen et al., 2013). This discrepancy might be a consequence of the interaction between environment and genes. Family studies are conducted amongst members of the same populations, whereas GWAS exploit data from individuals across populations. If genes can explain variance in fertility within but not between populations, heritability estimates based on different populations will be smaller than within populations (Manolio et al., 2009; Visscher et al., 2008). Third, Fisher's fundamental theorem of natural selection predicts at environmental equilibrium, close to zero additive genetic effects on fitness-related traits such as fertility, because genes that reduce fitness are not expected to have been passed on to the next generation (Fisher, 1930). Nevertheless, additive genetic influences on fertility are well established and a potential explanation is that the genes that are important for fertility differ across environments (Hughes \& Burleson, 2000). 
Twin and family designs cannot be used to answer the question of whether different genes are important for fertility across populations or birth cohorts, since relatives usually live in the same country and twins always have the same age. However, with the advent of molecular genetic data and complementary analytical techniques and software, it has become possible to examine the genetic material of unrelated individuals from different historical populations. Testing whether the same genes influence a trait across diverse environments has therefore become possible (Purcell et al., 2007; Visscher et al., 2014, 2010; Yang, Lee, et al., 2011; Yang et al., 2010). In this study, we exploit these advances for the first time, by empirically assessing whether genetic effects on fertility differ across geographical and historical environments.

We pooled a series of large datasets consisting of 31,396 unrelated ( second cousin, IBS $<0.05$, see Material and Methods) genotyped men $(n=10,489)$ and women ( $n=20,907)$ from six countries and seven study populations for analysis (for the US: HRS, ARIC; for the Netherlands: LifeLines; For Sweden: STR/SALT; for Australia: QIMR; for Estonia: EGCUT; for the UK: TwinsUK) who are assumed to have completed their reproductive period $\left(a g e_{\text {men }}>50 ; a g e_{\text {women }}>45\right)$. We first conducted a mega-analysis, which is based on individual information from different populations in contrast to a meta-analysis that uses summary statistics of analyses conducted within populations, and applied whole genome methods (Visscher et al., 2010; Yang et al., 2010) using GCTA software (Yang, Lee, et al., 2011) to estimate SNP-heritability $\left(h_{S N P}^{2}\right)$. SNP-heritability is the proportion of total phenotypic variance that is explained by common genome-wide SNPs. Based on a previous study using data from women from the Netherlands and the UK, we expect $h_{S N P}^{2}$ to be around 0.10 for number of children ever born and around 0.15 for AFB (Tropf, Stulp, et al., 2015).

Second, to investigate gene-environment interaction, we follow two strategies. The first one involves fitting multiple genetic relatedness matrices in our model, one global matrix for all individuals and more matrices indicating whether individuals lived in the same population and/or were part of the same birth cohort. The global matrix estimates the effects genes have across all environments, whereas the population/birth cohort specific matrices estimate context specific genetic effects (see Yang, Lee, et al., 2011 and Material and Methods for our specifications). The 
felix-tropf.com

second strategy involves fitting bivariate genetic models to investigate the moderating effect of the postponement transition on genetic effects on fertility (S. H. Lee et al., 2012 and Material and Methods for our specifications; see Visscher et al., 2014). This model allows us to estimate $h_{S N P}^{2}$ separately for different birth cohorts as well as the genetic correlation across them. To maximize power in these models, we divided all populations into two demographic birth cohorts. A central turning point in the reproductive environment of the 20 $0^{\text {th }}$ century occurred when AFB experienced a massive postponement of up to $4-5$ years in nearly all advanced societies, the socalled 'postponement transition' (Kohler, Billari, et al., 2002), or Second Demographic Transition (Balbo et al., 2013; Lesthaeghe, 1995; Mills et al., 2011; van de Kaa, 1987). The primary reasons proposed for fertility postponement have been women's increased educational attainment and their employment in the labour force, triggered by factors such as the availability of effective contraception (Balbo et al., 2013; Mills et al., 2011). Cultural transformations in terms of sexual freedom, family planning and the timing and role of children are also central (Lesthaeghe, 1995; van de Kaa, 1987). To investigate the moderating effect of fertility postponement we divide individuals into birth cohorts born either before or after this massive postponement in AFB in the past century (Balbo et al., 2013; Kohler, Billari, et al., 2002; Mills et al., 2011; Schmidt, Sobotka, Bentzen, \& Andersen, 2012).

\subsection{Data \& method}

\subsubsection{Data}

In this study we combined data from seven cohorts and six countries. For the US, we use data from ARIC, HRS, for Estonia from EGCUT, for Australia QIMR data from the Australia Twin and Family Register, for the Netherlands the LifeLines Cohort Study, for the United Kingdom TwinsUK and for Sweden the STR. All studies have received ethical approval. 


\begin{abstract}
ARIC
ARIC (Atherosclerosis Risk in Communities Study) is a community-based prospective cohort study of 15,792 adults, ages 45-64. Participants were identified by probability sampling from four U.S. communities (Forsyth County, North Carolina; Jackson, Mississippi; suburban Minneapolis, Minnesota; and Washington County, Maryland) and were enrolled between 1987 and 1989 (Investigators, 1989; Jackson et al., 1996; White \& Folsom, 1996).
\end{abstract}

HRS

The Health and Retirement Study (HRS) is an ongoing cohort study of Americans, with interview data collected biennially on demographics, health behavior, health status, employment, income and wealth, and insurance status. The first cohort was interviewed in 1992 and subsequently every two years, with 5 additional cohorts added between 1994 and 2010. The full details of the study are described in (Juster \& Suzman, 1995).

\title{
EGCUT
}

Estonian data come from of the Estonian Genome Center Biobank, University of Tartu (EGCUT, www.biobank.ee), a population-based database which comprises the health, genealogical and genome data of currently more than 51,530 individuals (Leitsalu et al., 2015). Each participant filled out a Computer Assisted Personal Interview including personal data (place of birth, place(s) of living, nationality etc.), genealogical data (family history, three generations), educational and occupational history and lifestyle data (physical activity, dietary habits, smoking, alcohol consumption, and quality of life). 


\section{QIMR}

Data for Australia was received from the Queensland Institute for Medical Research (QIMR). The participants were drawn from cohorts of adult twin families that have taken part in a wide range of studies of health and well-being via questionnaire and telephone interview studies, and recruitment was extended to their relatives (parents, siblings, adult children and spouses).

\section{LifeLines Cohort Study}

The LifeLines Cohort Study (Klijs et al., 2015) is a multi-disciplinary, prospective population-based cohort study from the Netherlands, examining in a unique three-generation design the health and health-related behaviours of 167,729 persons living in the North of The Netherlands, which includes genotype information from more than 13,000 unrelated individuals. It employs a broad range of investigative procedures in assessing the biomedical, socio-demographic, behavioural, physical and psychological factors which contribute to the health and disease of the general population, with a special focus on multi-morbidity and complex genetics.

\section{TwinsUK}

For the UK, we use data from TwinsUK, the largest adult twin registry in the country with more than 12,000 respondents (Moayyeri et al., 2013). The TwinsUK Study recruited white monozygotic (MZ) and dizygotic (DZ) twin pairs from the TwinsUK adult twin registry, a group designed to study the heritability and genetics of age-related diseases (www.twinsuk.ac.uk). These twins were recruited from the general population through national media campaigns in the UK and shown to be comparable to age-matched population singletons in terms of clinical phenotype and lifestyle characteristics.

\section{STR}


The Swedish Twin Registry (STR) was first established in the late 1950s to study the importance of smoking and alcohol consumption on cancer and cardiovascular diseases whilst controlling for genetic propensity to disease. Between 1998 and 2002, the STR conducted telephone interview screening of all twins born in 1958 or earlier regardless of gender composition or vital status of the pair. This effort is known as Screening Across the Lifespan Twin study (SALT). A subsample of SALT $(\approx 10,000)$ was genotyped as part of the TwinGene project (Benjamin et al., 2012; Lichtenstein et al., 2006) and we use the this information in the current study.

\section{Fertility trends}

Aggregate data to describe country specific fertility trends have been obtained from the Human Fertility Database (HFD, http://www.humanfertility.org/cgibin/main.php) and the Human Fertility Collection (HFC, http://www.fertilitydata.org/cgi-bin/index.php), where available. Both data collections are joint projects of the Max Planck Institute for Demographic Research (MPIDR) in Rostock, Germany and the Vienna Institute of Demography (VID) in Vienna, Austria. The projects provide access to detailed and high-quality data on period and cohort fertility. The HFD is entirely based on official vital statistics. The HFC incorporates a variety of valuable fertility data from diverse, not necessarily official, data sources. All data are freely available after registration. We focused on fertility information for cohorts that was aggregated for individuals older than 45 .

For the UK, official data on birth order have only been collected within marriage, and might be biased. We therefore relied on estimates from the Office for National Statistics, Cohort fertility, Table 2 (Office National Statistics, 2013). For Estonia, data on completed reproduction by age 45 was only available until the year 1962. For subsequent cohorts, however, there was an estimate of AFB available based on official statistics at the age of 40 . For Australia, no official data on a time series of cohort specific AFB was available, and the trends are based on the data used for analysis in this study (see Supporting Information 4-4 for distribution).

\section{Genotypes}


We received genotype data from all cohorts, which we imputed according to the 1000 genome panel - except for TwinsUK for which we already received the imputed data.

\section{Genetic-relatedness-matrix (GRM)}

To estimate the genetic relatedness-matrix (GRM), the HapMap3 imputation panel was used as a reference set as it was optimized to capture common genetic variation in the human genome (Consortium, 2010).We selected HapMap3 SNPs with an imputation score larger than 0.6 . For quality control (QC), we excluded the SNPs with a larger missing rate than 5 percent after merging, lower minor allele frequency than 1 percent, and which failed the Hardy-Weinberg equilibrium test for a threshold of $10^{-6}$. We merged the datasets subsequently applying QC again after merging each data set. 847,278 SNPs could be utilized to estimate the GRM between individuals. We used the software Plink (Purcell et al., 2007) for the quality control and merging of the datasets and GCTA (Yang, Lee, et al., 2011) to estimate the genetic relatedness matrix.

The GRM $A_{i k}$ is estimated for each pair of individuals $\mathrm{j}$ and $\mathrm{k}$ :

$$
A_{j k}=\frac{1}{N} \sum_{i=1}^{N} \frac{\left(x_{i j}-2 p_{i}\right)\left(x_{i k}-2 p_{i}\right)}{2 p_{i}\left(1-p_{i}\right)}
$$

where $x_{i j}$ and $x_{i k}$ is the number of copies of the reference allele for the $i^{\text {th }}$ SP of the $j^{\text {th }}$ or $k^{\text {th }}$ individual and $p_{i}$ is the frequency of the reference allele and $N$ the number of SNPs. If two individuals had a higher genetic relatedness than 0.05 , one was excluded from the analyses to avoid bias due to environmental confounders amongst close relatives.

\section{Phenotypes}

Number of children ever born was available for all cohorts, but in ARIC and TwinsUK, however, only for women. NEB measures number of children a woman has given birth to or a man has fathered. It was either directly asked or we constructed it from questions on the date of birth of each child. 
The measure is not perfectly harmonized across cohorts because some questionnaires explicitly exclude still-births (HRS, ARIC) while others remain undefined (TwinsUK asked in some waves: "How many children have you given birth to?"; EGCUT asked: "Do you have any biological children?", and subsequently: "Fill in their names, gender and date of birth). In STR, LifeLines, QIMR as well as most of the waves of the TwinsUK, information on both the date of birth and death of the child was asked. In LifeLines and TwinsUK, we compared the live birth measure with number of children ever born and, as expected given the diminishing mortality rate in both datasets, less than 0.2 percent of the children had not reached reproductive age, and the correlation of number of children ever born and number of children reaching reproductive age was $>0.98$. We therefore expect no large bias due to the fact that in some countries still-births are excluded.

Furthermore, the questionnaires were heterogeneous with respect to the maximum number of children that could be named. However, within each cohort, the maximum number of children in the respective questionnaires has never been named more often than in 0.5 percent of the cases and we do not anticipate that our results are influenced by this.

Information on AFB was available in all cohorts except for ARIC and the HRS. It was asked directly (in TwinsUK), or was constructed using the date of birth of the oldest child and the year of birth of the respondent.

Since fertility is strongly age dependent, we focus on women with a completed fertility history in reference to the phenotype. In general, the end of women's reproductive lifespan occurs around the age of 45, and the end of men's at the age of 50. Thus, we only included individuals beyond those ages in our analyses. Furthermore, in vitro fertilization (IVF) - which is often related to twinning and multiple births - can bias results if IVF compensates for genetically based infertility. However, in our TwinsUK sample, only 60 women reported using IVF, who we did not include in the final analyses. For all models, both phenotypes were standardized (Z-transformed) by cohort, year of birth and sex. 


\subsubsection{GREML Models}

\section{Common SNP heritability estimates (Model 1)}

The genetic component underlying a trait is commonly quantified in terms of SNP-heritability $\left(h_{S N P}^{2}\right)$ as the proportion of the additive genetic variance explained by common SNPs across the entire genome over the total phenotypic variance $\left(\sigma_{P}^{2}\right)$ of the trait:

$$
h_{S N P}^{2}=\frac{\sigma_{g}^{2}}{\sigma_{P}^{2}} .
$$

The phenotypic variance is the sum of additive genetic and environmental variance, i.e. $\sigma_{P}^{2}=\sigma_{g}^{2}+\sigma_{e}^{2}$ where $\sigma_{g}^{2}$ is the additive genetic variance explained by all SNPs across the genome and $\sigma_{e}^{2}$ is the residual variance.

The methods we applied have been detailed elsewhere (S. H. Lee et al., 2012; Visscher et al., 2014, 2010; Yang, Lee, et al., 2011; Yang et al., 2010). Briefly, we applied a linear mixed model

$$
\mathbf{y}=X \boldsymbol{\beta}+\mathbf{g}+\mathbf{e}
$$

where $\mathbf{y}$ is an Nx1 vector of dependent variables, $N$ is the sample size, $\beta$ is a vector for fixed effects of the overall mean (intercept), $\mathbf{g}$ is the $\mathrm{Nx} 1$ vector with each of its elements being the total genetic effect of all SNPs for an individual, and $\mathbf{e}$ is an $\mathrm{Nx} 1$ vector of residuals. The variance covariance matrix of the observed phenotypes is:

$$
\mathbf{V}=\mathbf{A} \sigma_{g}^{2}+\mathbf{I} \sigma_{e}^{2}
$$

We have $\mathrm{g} \sim N\left(0, \mathbf{A} \sigma_{g}^{2}\right)$ and $\mathrm{e} \sim N\left(0, \mathbf{I} \sigma_{e}^{2}\right)$, $\mathbf{A}$ is the genetic relationship matrix (GRM) estimated from SNPs and I is an identity matrix. The variance components are estimated using the restricted maximum likelihood (REML) approach.

\section{Genes x Population (Model 2)}


The genes $x$ demographic birth cohort interaction model is a joint model estimating global genetic effects for the fertility traits, effective between and within samples $\left(\sigma_{g}^{2}\right)$ and the averaged additional genetic effects within cohorts $\left(\sigma_{g x p}^{2}\right)$ :

$$
\mathbf{V}=\mathbf{A} \sigma_{g}^{2}+\mathbf{A}_{\mathbf{g x p}} \sigma_{g x p}^{2}+\mathbf{I} \sigma_{e}^{2}
$$

where $\mathbf{A}$ is the genetic relatedness matrix and $\mathbf{A}_{\text {gxp }}$ is a matrix only with values for pairs of individuals within populations 1-7:

$$
\begin{aligned}
& \mathbf{A}=\left[\begin{array}{l}
A_{p 1 p 1} A_{p 2 p 1} A_{p 3 p 1} A_{p 4 p 1} A_{p 5 p 1} A_{p 6 p 1} A_{p 7 p 1} \\
A_{p 1 p 2} A_{p 2 p 2} A_{p 3 p 2} A_{p 4 p 2} A_{p 5 p 2} A_{6 p 2 p} A_{p 7 p 2} \\
A_{c 1 p 3} A_{p 2 p 3} A_{p 3 p 3} A_{p 4 p 3} A_{p 5 p 3} A_{p 6 p 3} A_{p 7 p 3} \\
A_{c 1 p 4} A_{p 2 p 4} A_{p 3 p 4} A_{p 4 p 4} A_{p 5 p 4} A_{p 6 p 4} A_{p 7 p 4} \\
A_{c 1 p 5} A_{p 2 p 5} A_{p 3 p 5} A_{p 4 p 5} A_{p 5 p 5} A_{p 6 p 5} A_{p 7 p 5} \\
A_{p 1 p 6} A_{p 2 p 6} A_{p 3 p 6} A_{p 4 p 6} A_{p 5 p 6} A_{p 6 p 6} A_{p 7 p 6} \\
A_{p 1 p 7} A_{p 2 p 7} A_{p 3 p 7} A_{p 4 p 7} A_{p 5 p 7} A_{p 6 p 7} A_{p 7 p 7}
\end{array}\right] \\
& \mathbf{A}_{\boldsymbol{g x p}}=\left[\begin{array}{ccccccc}
A_{p 1 p 1} & 0 & 0 & 0 & 0 & 0 & 0 \\
0 & A_{p 2 p 2} & 0 & 0 & 0 & 0 & 0 \\
0 & 0 & A_{p 3 p 3} & 0 & 0 & 0 & 0 \\
0 & 0 & 0 & A_{p 4 p 4} & 0 & 0 & 0 \\
0 & 0 & 0 & 0 & A_{p 5 p 5} & 0 & 0 \\
0 & 0 & 0 & 0 & 0 & A_{p 6 p 6} & 0 \\
0 & 0 & 0 & 0 & 0 & 0 & A_{p 7 p 7}
\end{array}\right]
\end{aligned}
$$

\section{Genes x Demographic birth cohort (Model 3)}

The genes $\mathrm{x}$ demographic birth cohort interaction model is a joint model estimating the universal genetic effects for the traits, effective between and within samples $\left(\sigma_{g}^{2}\right)$ and the averaged additional genetic effects within defined birth cohorts $\left(\sigma_{g x b}^{2}\right)$ :

$$
\mathbf{V}=\mathbf{A} \sigma_{g}^{2}+\mathbf{A}_{\mathbf{g x d}} \sigma_{g x d}^{2}+\mathbf{I} \sigma_{e}^{2}
$$

whereas $\mathbf{A}$ is the genetic relatedness matrix and $\mathbf{A}_{\mathbf{g x d}}$ is a matrix only with values for pairs of individuals within the same demographic birth cohorts b1-2: 
felix-tropf.com

$$
\mathbf{A}_{\mathbf{g x b}}=\left[\begin{array}{cc}
A_{d 1 d 1} & 0 \\
0 & A_{d 2 d 2}
\end{array}\right]
$$

\section{Genes x Population x Demographic birth cohorts (Model 4)}

Finally, we applied a model including both interaction terms from above and an additional interaction term $\mathbf{A}_{\mathbf{g x c x b}}$ which is 0 for all pairs of individuals living in different time periods or in different cohorts

$$
\mathbf{V}=\mathbf{A} \sigma_{g}^{2}+\mathbf{A}_{\mathbf{g x p}} \sigma_{g x p}^{2}+\mathbf{A} \sigma_{g x d}^{2}+\mathbf{A}_{\mathbf{g x p x d}} \sigma_{g x p x d}^{2}+\mathbf{I} \sigma_{e}^{2}
$$

whereas $\mathbf{A}$ is the genetic relatedness matrix, $\mathbf{A}_{\mathbf{g x p}}$ is a matrix only with values for pairs of individuals within populations 1-7 (Model 2), $\mathbf{A}_{\mathbf{g x d}}$ is a matrix only with values for pairs of individuals within the same demographic periods b1-2 (Model 3) and $\mathbf{A}_{\mathbf{g x p x d}}$ is a matrix only with values for pairs of individuals with both the same demographic periods and the same populations.

\section{Bivariate Model}

For bivariate analyses (S. H. Lee et al., 2012; Visscher et al., 2014), we split the data into individuals born before and after the turning point in fertility postponement in AFB (see also Supporting Information 4-2 \& 4-7). Based on Model 2, we estimate a bivariate model with two GRMs:

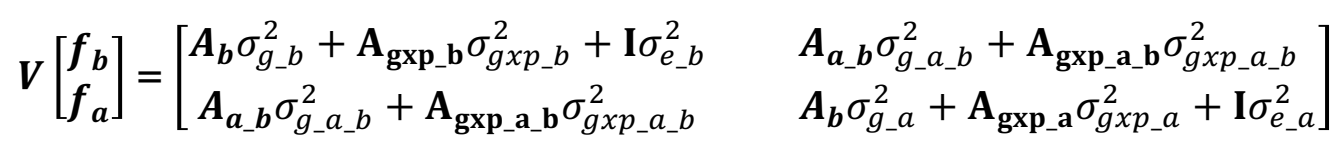

where as $\boldsymbol{f}_{\boldsymbol{b}}$ and $\boldsymbol{f}_{\boldsymbol{a}}$ are vectors of length $N_{b}$ and $N_{a}$ of fertility phenotypes (NEB or AFB) of individuals born before or after the postponement transition started, with $\mathrm{N}$ being the respective sample size of the subsets. Variance components refer to those from Model 2, whereas the lower index _b indicates that they are estimated in the subset of individuals born before and index _a born after the start of the postponement transition. The index _ b_a denominates the covariance of variances components across subsets. The correlation of the genetic factors are estimated as: 


$$
r_{\sigma_{g x p_{-} \_b}^{2}}=\sigma_{g x p_{-} a \_b}^{2} / \sqrt{\sigma_{g x p_{-} a}^{2} * \sigma_{g x p_{-} b}^{2}}
$$

and

$$
r_{\sigma_{g_{-} a_{-} b}^{2}}=\sigma_{g_{-} a \_b}^{2} / \sqrt{\sigma_{g_{-} a}^{2} * \sigma_{g_{-} b}^{2}}
$$

\subsection{Results}

\subsubsection{Descriptive findings}

The descriptive statistics for NEB and AFB for all populations under study (LifeLines, TwinsUK, STR, Estonia, HRS, ARIC and QIMR) as well as the pooled data separate for men and women can be found in Supporting Information 4-1. The participants were born between 1903 and 1967. The mean number of children per woman is 2.0 in Estonia, Sweden and the UK and 3.3 in Australia. For men, the lowest reported number of children is in Sweden and Estonia with around 1.9 children per man, and is the highest in Australia at around 3.4. AFB was available for the Netherlands, UK, Sweden, Estonia and Australia. For both men and women, it was lowest in Estonia with an average of 24.6 for women and 27.7 for men and highest in Australia with 26.7 and 29.8 for men. Individuals who start reproducing at a later age have fewer children, with correlations between NEB and AFB ranging between -0.24 (Netherlands) and -0.38 (Australia; Supporting Information 4-2). This pattern is less consistent across countries; for example in Australia, the highest fertility levels are observed, despite there being the highest AFB. This reflects heterogeneity in fertility levels across countries, with Australia having traditionally higher fertility levels than other Western countries (for a trend comparison of the total fertility rate across countries see Supporting Information 4-3).

\subsubsection{Demographic Trends}

Figure 4-1 shows the trends in AFB during the $20^{\text {th }}$ century for the countries in our study based on population data if available (see Data \& Methods for details). We observe the well-established U-shaped pattern of AFB of a falling AFB in the first 
felix-tropf.com

half of the $20^{\text {th }}$ century followed by a turning point and upturn in the trend of AFB towards older ages. This postponement transition in fertility timing was accompanied by a strong drop in completed fertility in most countries (Sobotka, 2004).

Sociocultural and technological changes, such as the introduction of effective contraception, educational expansion or changing norms in reference to sexuality and family planning, have largely driven these trends (Balbo et al., 2013; Mills et al., 2011). These environmental changes occurred in specific time periods in each country. In order to test for gene-environment interaction in our analyses, we split the data into birth cohorts born before and after the turning point of fertility postponement to reduce environmental heterogeneity amongst the individuals who are members of the same birth cohort. This turning point differs across countries (Figure 4-1), with Australia having the earliest onset of postponement (1939) and Estonia the latest (1962; see Supporting Information 4-5 for all turning points and details). Differences in the onset of the postponement transition are well established and can be due to political factors. This is true in the case of Estonia, for example, where early AFB had been strongly promoted through political incentives when the country was still of the Soviet Union prior to 1990 (Katus, Puur, \& Põldma, 2004).

\subsubsection{Genetic effects on fertility from the whole genome}

\section{Model 1: SNP heritability of AFB and NEB across environments}

Not taking environmental differences into account, SNP based heritability $\left(h_{\text {SNP }}^{2}\right)$ is significant and low for number of children ever born and age at first birth (Table 4-1). For NEB, $\mathrm{h}_{\mathrm{SNP}}^{2}$ is $0.038\left(\mathrm{SE}=0.0097, \mathrm{p}\right.$-value $=2.0 \times 10^{\text {s) }}$ ) and for AFB it is $0.053(\mathrm{SE}=0.019 \mathrm{p}$-value $=0.0020$; these estimates are based on the full genetic relatedness matrix - see Material and Methods). These findings mean that around four percent of the variance in NEB and around five percent in AFB can be attributed to common, additive genetic effects in the pooled data. These estimates are much lower than those reported in other studies (Tropf, Stulp, et al., 2015).

Model 2: Genes x population interaction ( $\mathrm{g} \times \mathrm{p}$ ) 
A potential reason why estimates are lower than expected is that the SNPs important for fertility have different effects across environments. Model 2 therefore adds an interaction term to Model 1 that captures the influence of genetic variance on fertility only within populations. The gene-population interaction models for NEB and AFB show that shared genetic effects across populations are much lower than genetic effects within populations. With respect to NEB, the shared genetic effects across populations are negligible $(0.0070, \mathrm{SE}=0.011$, $\mathrm{p}$-value $=0.26)$, whereas within populations additional additive effects are estimated to be 0.15 (SE $=0.024$, pvalue $\left.=6.0 \times 10^{-12}\right)$.

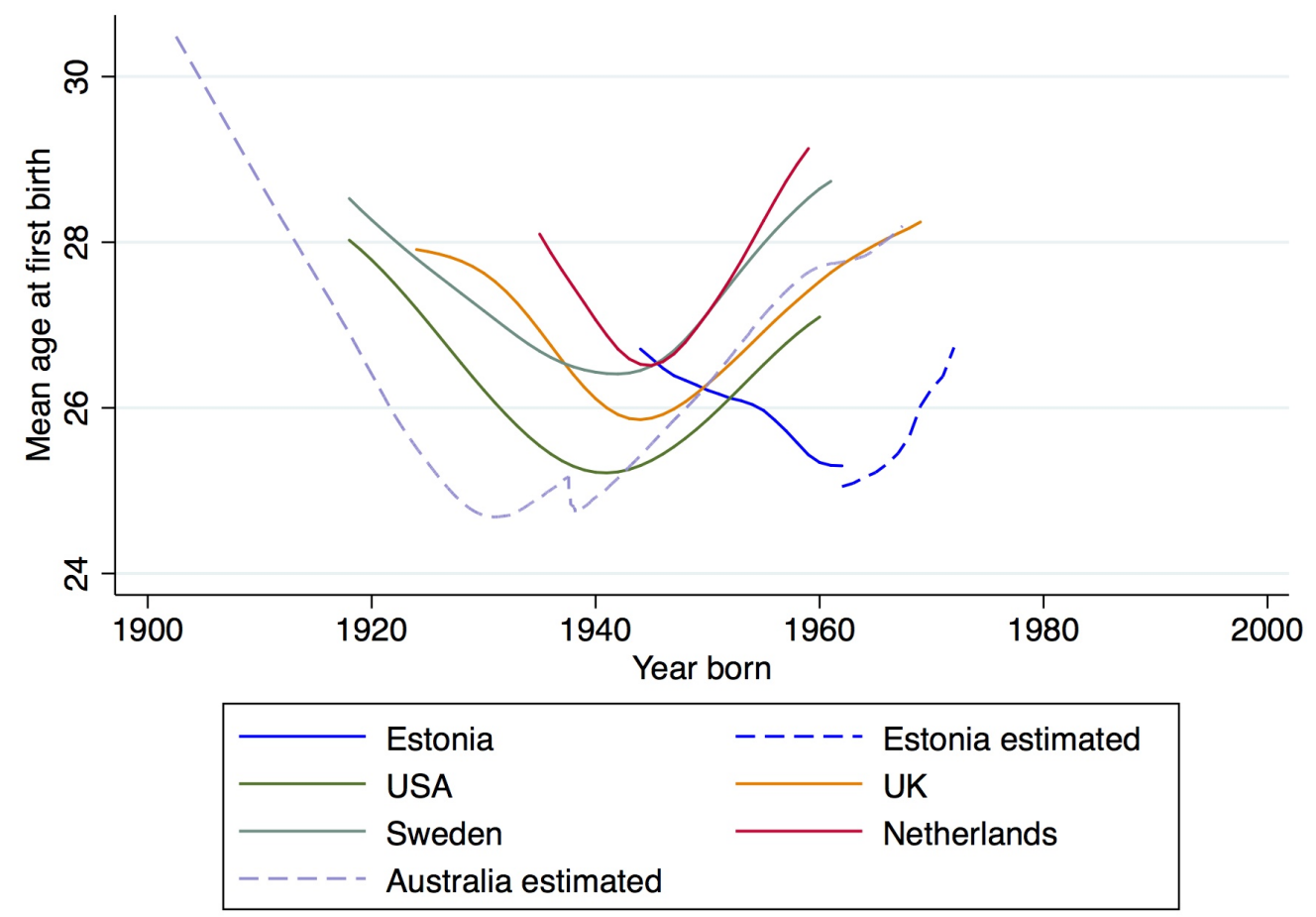

Figure 4-1 Trends in age at first birth in cohorts from the US, UK, Sweden, the Netherlands, Estonia and Australia (1903-1970).

Note: Trends in the mean age at first birth are moving averages based on aggregated data obtained from Human Fertility Database and the Human Fertility Collection (for details see Data and Methods). For Australia, no official data has been available and the trends have been estimated from the QIMR dataset. See Supporting Information 4-4 for the birth cohort specific average in the QIMR data.

Source: See Methods section for details 
felix-tropf.com

The same applies to AFB, where shared genetic effects are estimated to be only 0.024 $(\mathrm{SE}=0.022$, $\mathrm{p}$-value $=0.14)$, whereas the within population effect is $0.10(\mathrm{SE}=0.039$, p-value=0.0037; Table 4-1; Model 2). These results show that there is little overlap in SNPs that influence fertility across populations, and that most of the SNPs influencing fertility are population specific.

\section{Model 3: Genes x demographic birth cohort ( $\mathrm{x}$ d)}

Similar to the case of Model 2, in which we modelled population specific effects, we also examined whether there were genetic influences on fertility specific to particular birth cohorts. We find that there is additional genetic variance explanation for individuals who live in the same demographic cohort. While $\mathrm{h}_{\mathrm{SNP}}^{2}$ for all birth cohorts is estimated at zero for both NEB (SE $=0.013$, p-value $=0.50)$ and AFB $(S E=0.03, p$-value $=0.35)$, for individuals living in the same demographic cohort there is a significant additional genetic variance component of 0.097 (SE = $0.017, \mathrm{p}$-value $\left.=3.3 \times 10^{16}\right)$ for NEB and $0.084\left(\mathrm{SE}=0.031, \mathrm{p}\right.$-value $\left.=4.6 \times 10^{4}\right)$ for $\mathrm{AFB}$ (Table 4-1; Model 3). Thus, as in the case of the different populations, we find that SNPs influencing fertility traits are specific to particular birth cohorts.

\section{Model 4: Genes x population $x$ demographic birth cohort $(g \times p \times d)$}

Including a gene-environment interaction term that takes into account both the population and the demographic cohort simultaneously (Model 4), we observe that for NEB both the interaction with demographic cohort $(0.064, \mathrm{SE}=0.020$, pvalue $\left.=5.9 \times 10^{4}\right)$ and the interaction with population and demographic cohorts $(0.085, \mathrm{SE}=0.045, \mathrm{p}$-value $=0.0030)$ are significant. This suggests that living in the same demographic cohort increases $h_{\mathrm{SNP}}^{2}$ independent of whether individuals live in the same population, and that living in the same population and the same demographic period further increases $\mathrm{h}_{\mathrm{SNP}}^{2}$. For AFB, $\mathrm{h}_{\mathrm{SNP}}^{2}$ is only significantly different from zero for individuals living in the same population and demographic cohort $(0.18, \mathrm{SE}=0.077, \mathrm{p}$-value $=0.0032)$.

\section{Overall SNP based heritability for each model}


Subsequently, overall heritability estimates were calculated as the sum of the different components of each model. This was done in order to examine the increase in heritability estimates when including the different interaction terms (See Figure 42 corresponding to Supporting Information 4-6). The overall $\mathrm{h}_{\mathrm{SNP}}^{2}$ for NEB increases almost fivefold, from $0.04(\mathrm{SE}=0.01$; Model 1) to $0.22(\mathrm{SE}=0.026)$, when population and demographic cohort are taken into account. For AFB, the trend is very similar, with an $h_{\text {SNP }}^{2}$ of $0.053(S E=0.019)$ in the baseline Model 1, and $0.19(\mathrm{SE}=0.039)$ in the genes $x$ population $x$ demographic cohort interaction model-where population and demographic cohort are taken into account.

\section{Sensitivity analysis: Genes x Sex}

The analyses presented are based on pooled datasets of men and women. However, two data sources contain (almost) only women (TwinsUK and ARIC). To the extent that different genes influence fertility across sexes, this might drive the observed differences across populations. We therefore conducted a sensitivity analysis extending Model 3 to a genes x population $x$ sex interaction model. We find that considering sex-differences does not significantly improve the model fit ( $p$ value for AFB 0.5, for NEB 0.093). We are therefore confident that our findings do not result from sex-differences (Supporting Information 4-8).

We estimated a complementary bivariate model based on Model 2, by splitting data for demographic cohorts (see Material and Methods), which allows us to estimate genetic effects across $\left(\sigma_{g}^{2}\right)$ and within $\left(\sigma_{g x p}^{2}\right)$ populations separately for different demographic birth cohorts, and thereby investigate whether genetic effects are correlated across demographic birth cohorts. Table 4-2 shows that $\sigma_{g x p}^{2}$ estimates for NEB within populations are significant for both demographic cohorts before $\left(\sigma_{g x p}^{2} / \sigma_{p}^{2}=0.15, \mathrm{SE}=0.039, \mathrm{p}\right.$-value $\left.=9.6 \times 10^{-}\right)$and after $\left(\sigma_{g x p}^{2} / \sigma_{p}^{2}=0.13, \mathrm{SE}=0.048\right.$, p-value $=0.0010)$ fertility postponement. It also shows a positive correlation of genetic effects with NEB across demographic cohorts and within populations (1.00, $\mathrm{SE}=0.35, \mathrm{p}$-value $=1.3 \times 10^{-5}$ ). In Model 4 of Table $4-1$, this remained suggestive, since the genetic effects within populations but shared across demographic cohorts $\left(\sigma_{g x p}^{2} / \sigma_{P}^{2}\right)$ were non-significant $(0.073, \mathrm{SE}=0.036, \mathrm{p}$-value $=0.18)$. 


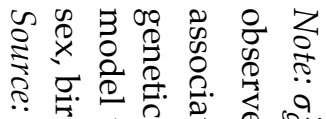

के $\begin{aligned} & 0 \\ & 0\end{aligned}$

造芦.

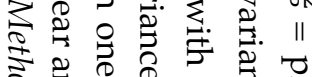

के

क 8 के

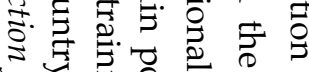

क.

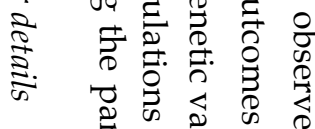

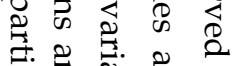

己े

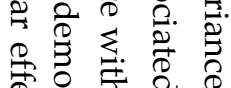

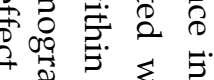

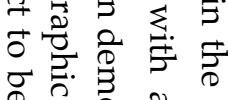

ㄷ. 잉 ڤ

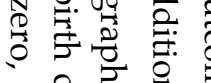

율

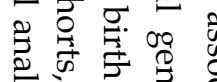

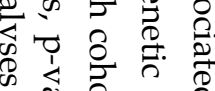

हैं है

क की

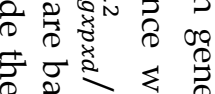

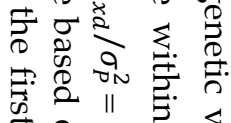

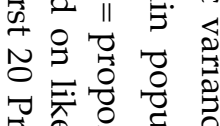

․ㅊㅇㅠ

స.

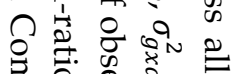

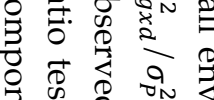

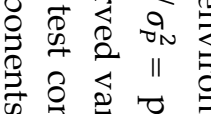

क

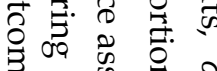

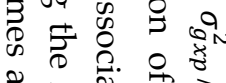

藏焉离

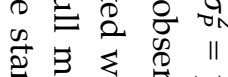

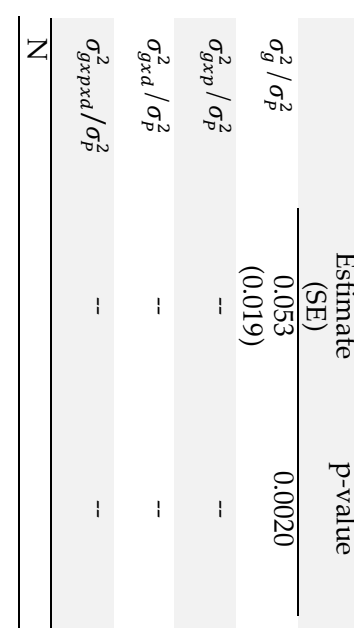

3
$\frac{1}{0}$
0
0

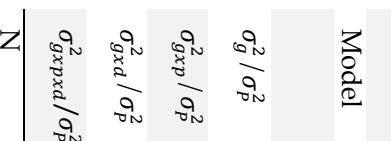

룰 㫄

i

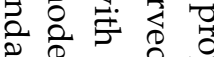

ใ 207

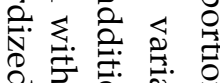

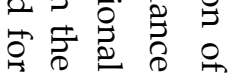

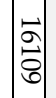

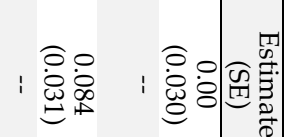

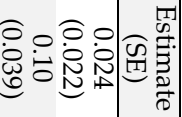

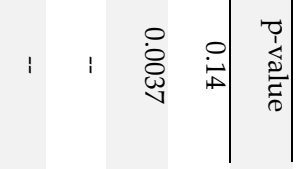

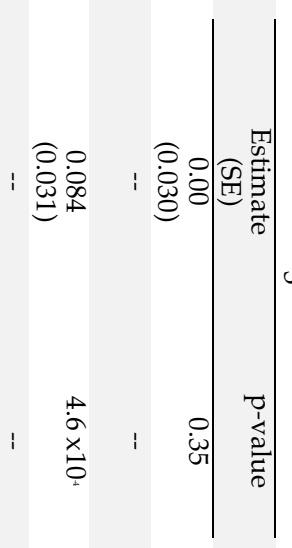

幽

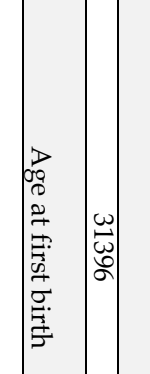

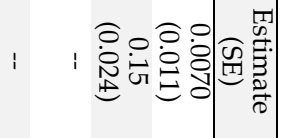

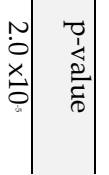

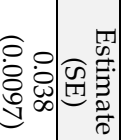

ב.

$\approx \stackrel{\circ}{7}$

萂

丞异

蛋署

క⿱丶万仒

है

$\stackrel{0}{9}$

0
3
0
0
0
0
0
0
0
0
0
0
0
0
0
0
0
0
0

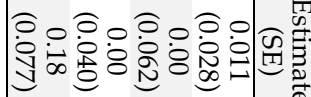

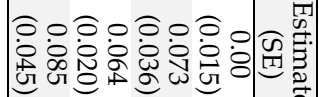

옹

:

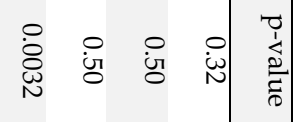

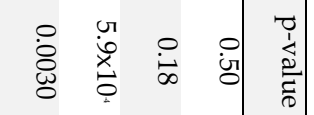

吕 


\section{Bivariate analysis}

The bivariate model for the AFB finds some evidence that in both demographic cohorts genetic effects are observed (before fertility postponement $0.099, \mathrm{SE}=0.073, \mathrm{p}$-value $=0.083$; after fertility postponement $0.13, \mathrm{SE}=0.074, \mathrm{p}$ value $=0.070)$, although these effects were marginally significant. However, there is no evidence that genetic effects correlate across demographic birth cohorts $(0.11, \mathrm{SE}$ $=0.59, \mathrm{p}$-value $=0.27)$, which is well in line with the null-estimate $\sigma_{g x p}^{2} / \sigma_{P}^{2}(0.00, \mathrm{SE}$ $=0.062, \mathrm{p}$-value $=0.50)$. Genetic effects shared across all populations $\left(\sigma_{g}^{2}\right)$ are only significant for NEB and birth cohorts born before fertility postponement $\left(\sigma_{g}^{2} / \sigma_{P}^{2}=\right.$ $0.031, \mathrm{SE}=0.018, \mathrm{p}$-value 0.042 ). By contrast, they are non-significant for younger cohorts and for AFB.

\subsection{Discussion}

Using data from seven populations and six countries, we demonstrate that genetic effects on fertility outcomes -number of children ever born (NEB) and age at first birth (AFB) - differ across temporal and spatial environments. For NEB, genetic effects within populations are stronger than across populations, but correlate between individuals who were born before or after the turning point in fertility postponement during the $20^{\text {th }}$ century. For AFB, genetic effects are only significant if individuals live in the same demographic cohort and the same population. Neither the full gene-environment interaction model (Model 4) nor the bivariate analyses provide evidence for shared genetic effects for each phenotype across populations and demographic birth cohorts. Our results show that different SNPs are associated with fertility traits in different populations and birth cohorts, and that very few genetic effects are consistently related to these traits across populations and cohorts. Our results uncover a strong interplay between genetic and environmental factors influencing human fertility. 


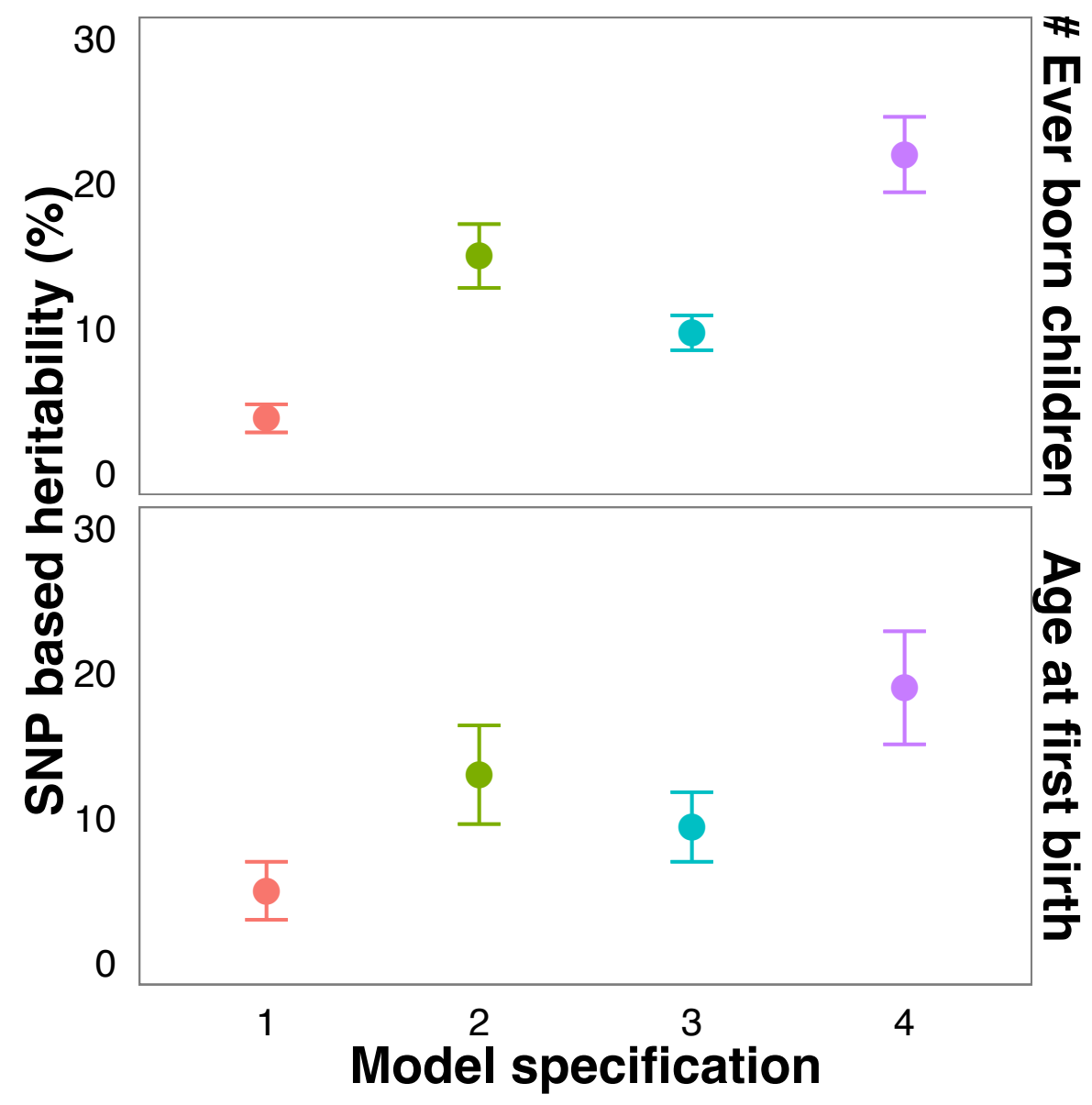

Figure 4-2 Bar Charts of the SNP-heritability estimates in number of children ever born (NEB) and age at first birth (AFB) for the different model specifications from Table 4-1

Note: SNP-heritability as the sum of genetic variance over the total variance in Model specification $1=$ amongst all individuals, 2 = amongst individuals living within the same population, $3=$ amongst individuals living within the same demographic birth cohort born either before or after fertility postponement, 4 = amongst individuals living in the same population and demographic birth cohort, dots = estimate, lines = estimate \pm 1 SE, The corresponding table to Figure 4-2 an be found in SI 4-6. 
Quantitative geneticists have been puzzled by low heritability estimates based on GWAS findings and whole-genome methods such as GREML, describing the phenomenon as the problem of 'missing heritability' (Manolio et al., 2009). Previous attempts to explain missing heritability partly by non-additive genetic effects remain largely empirically untested (Zuk \& Hechter, 2012) or find only little support (Zhu et al., 2015). Our findings of strong gene-environment interaction imply that the detection of genetic variants associated with fertility traits is a major challenge when using meta-analyses of GWAS on individuals from different populations. Likewise, predictions out of the discovery sample might not be straightforward to test, because discovered SNPs might have different effects in different samples.

In addition, our findings imply that lower heritability estimates from GWAS studies, as compared to GREML approaches or family studies, might be due to the fact that genetic effects are (to some extent) not universal, but rather context specific. In the model considering gene-environment interaction across population and demographic cohort, we report heritability findings of 0.22 for NEB and 0.19 for AFB (see Figure 4-2 and Supporting Information 4-8), which are fourfold higher than across all contexts and approach heritability estimates from family models (Mills \& Tropf, 2016; Polderman et al., 2015). It is therefore vital to understand the cultural and environmental factors that interact with human fertility as well as their origins across (family) environments in order-for example—to define missing heritability or validate the findings from twin studies. It should be noted that our findings are probably linked to the strong behavioural and social nature of fertility, which might be more sensitive to cultural and societal influence than morphological traits. A recent investigation by Yang et al. (2015) shows that missing heritability for the anthropometric traits height and body mass index is negligible when using whole genomic sequencing data in a new GREML model, and assuming that family models overestimate heritability.

Demonstrating that genetic effects on fertility outcomes differ across environments, our study contributes substantially to our current knowledge of the genetic architecture of human reproduction. Previous twin studies show for several countries and birth cohorts that fertility outcomes such as NEB and AFB are genetically influenced (Kohler et al., 2006; Mills \& Tropf, 2016). However, it 
remained unclear whether the same genes are associated with fertility across environments. Using molecular genetic data and GREML methods (Visscher et al., 2010; Yang, Lee, et al., 2011; Yang et al., 2010), we were able to study the genetic material of individuals across diverse environment,s and found that common SNPs explain substantially more variance within than between countries and birth cohorts for fertility traits.

Previous twin and family studies furthermore suggest that the heritability of fertility traits can change across time and space (Bras et al., 2013; Briley et al., 2015; Kohler et al., 1999; Tropf, Barban, et al., 2015). However, these differences could not be statistically validated. In the current study, we proposed a multi-matrix approach to test for gene-environment interaction but also applied bivariate GREML models across birth cohorts (S. H. Lee et al., 2012; Visscher et al., 2014). Bivariate GREML models allow estimation of SNP-heritability within two independent samples as well as the genetic correlation across them. We cannot confirm the suggestion that the level of heritability changed over time, but find that heritability levels are comparable before and after the strong fertility postponement during the past century.

Different levels of heritability have also been reported across countries (Mills \& Tropf, 2016). Our multi-matrix GREML approach distinguishes between pairs of individuals who are living in the same or in different populations. The resulting within population estimate is therefore an average across all populations and we cannot compare different levels of heritability across populations. A more desirable study design would be a multivariate genetic modelling approach (similar to the one we presented in a bivariate design) to investigate differences across demographic birth cohorts. However, this approach was not possible in our study due to small sample sizes in each population, and consequent lack of statistical power (Visscher et al., 2014). However, it might become feasible in the future with better data availability. 


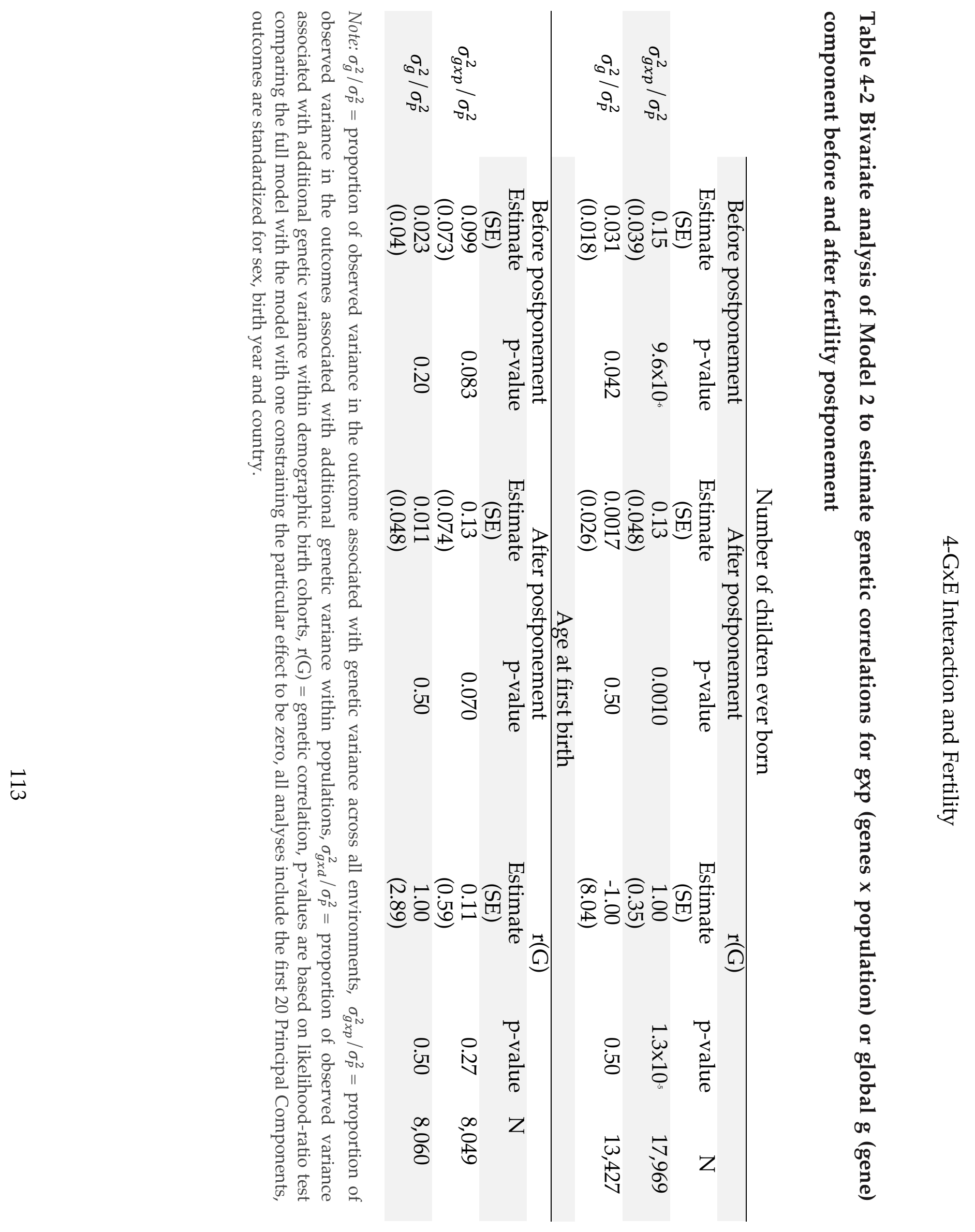


felix-tropf.com

Our findings are of interest to scientists within the medical, biological and social sciences alike (Kohler et al., 2006; Mills \& Tropf, 2016; Montgomery et al., 2014; Stearns et al., 2010). Research has successfully identified genetic variants associated with reproductive diseases and traits (Montgomery et al., 2014). However, it remains unknown how these affect realized fertility. We find no evidence that genetic effects underlying fertility in one country predict fertility outcomes in another one. Instead, genetic effects on fertility outcomes are strongly dependent on an individual's environment. Recently, social scientists have made large efforts to integrate molecular genetics into their research (Benjamin et al., 2012; J Bongaarts, 1983; Briley et al., 2015; Kohler et al., 2006; Mills \& Tropf, 2016; Rietveld et al., 2014; Rietveld, Cesarini, et al., 2013; Rietveld, Medland, et al., 2013; Tropf, Stulp, et al., 2015). However, when it comes to reproductive health, environmental factors are also likely to be critical in understanding how genetic effects on fecundity and infertility may be modified.

For evolutionary biologists, our findings have at least two important implications. First, the number of children ever born has been used as a proxy for fitness, given the diminishing child mortality rate in contemporary societies (Byars et al., 2010; Stearns et al., 2010; Tropf, Stulp, et al., 2015). Additive genetic variance therefore indicates natural selection under environmental equilibrium within populations if all else being equal, genes that lead to a higher number of offspring will have a higher frequency in future generations. Due to natural selection, Fisher predicted additive genetic variance in fertility to be (close to) zero in the absence of gene environment interaction, since genes that reduce fitness do not tend to be passed on to the next generation, thereby becoming lower in frequency (Fisher, 1930). Nevertheless, we find significant additive genetic influences on fitness traits such as NEB and AFB - substantial yet lower than heritabilities observed for morphological traits such as height (Mousseau \& Roff, 1987; Polderman et al., 2015; Tropf, Stulp, et al., 2015; Visscher et al., 2008). Finding significant genetic influences on these proxies of fitness suggests that, along with sociocultural changes surrounding fertility, genetic variants under selection have also changed (Briley et al., 2015; Courtiol, Rickard, \& Lummaa, 2013; Hughes \& Burleson, 2000; Kohler et al., 1999, for review see 2006; Kohler, Rodgers, et al., 2002; for review see Mills \& Tropf, 2016; for comment see Milot \& Pelletier, 2013; Tropf, Barban, et al., 2015). Gene- 
environment interaction can explain why we find additive genetic variance in fitness related traits despite natural selection.

Second, previous research has uncovered ongoing natural selection in contemporary societies (Bolund, Hayward, Pettay, \& Lummaa, 2015; Byars et al., 2010; Milot et al., 2011; Stulp et al., 2015; Tropf, Stulp, et al., 2015), and has even attempted to forecast changes in traits such as height and blood pressure across generations (Byars et al., 2010). In order for one to make valid evolutionary predictions about observable changes in traits across generations due to natural selection, fertility needs to be consistently heritable, the same genes need to be under selection across generations, and the direction of the selection needs be similar. Our results point to moderate genetic influences on fertility within populations, indicating potentially ongoing human evolution. However, this potential is limited in at least two ways. First, genetic effects on fertility strongly differ across countries and therefore may lead to heterogeneity across human populations rather than to universal changes in humans. Second, the finding that genetic effects underlying proxies of fitness vary so markedly across time periods suggests that substantial caution is needed when making long-term evolutionary predictions.

For social scientists, genetic influences had been originally thought of as biological constraints on human reproductive behavior (J Bongaarts, 1983). Yet some previous studies showed that genetic predispositions may underlie decision making processes governing fertility timing and motivation (W B Miller, 1992; Pasta \& Miller, 2000; Rodgers, Kohler, et al., 2001; Tropf, Barban, et al., 2015). Some suspect that genetically based behavioural and psychological traits have become more important than physiological ones in the recent past (Briley et al., 2015; Kirk et al., 2001; Rodgers, Kohler, et al., 2001; Udry, 1996). This hypothesis remains to be tested, but our results confirm that genetic influences on fertility have evolved with social changes in the reproductive environment and therefore underscore the need to integrate social factors into genetic research on fertility.

Overall, our study uncovers great challenges for investigations into the genetic architecture of fertility, which can only be overcome by interdisciplinary work between both social scientists and geneticists using ever larger datasets, with combined information from genetics and social surveys (Stearns et al., 2010). 



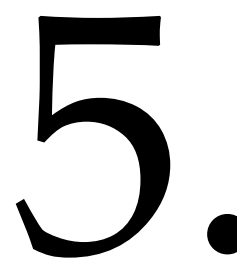

\section{Is the association between education and fertility postponement causal? The role of family background factors ${ }^{9}$}

Abstract: A large body of literature shows a positive relationship between education and age at first birth. However, this relationship may in part be spurious due to family background factors that cannot be controlled for in most research designs. We investigate to what extent education is causally related to later age at first birth in a large sample of female twins from the UK $(\mathrm{N}=2,752)$. We present novel estimates using within-identical twin and biometric models. Our findings show that one year of additional schooling is associated with about half a year later age at first birth in OLS models. This reduced to only 1.5 months for the within-identical twin model that controls for all shared family background factors (genetic and family environmental). Biometric analyses reveal that it is mainly influences of the family environment - not genetic factors - that cause spurious associations between education and age at first birth. Lastly, we demonstrate using data from the Office for National Statistics that only 1.9 months of the 2.4 years of fertility postponement for birth cohorts 1944-1969 could be attributed to educational expansion based on these estimates. We conclude that (the rise in) educational attainment alone cannot explain differences in fertility timing (between cohorts).

9

This Chapter is based on an article by Tropf F. C. \& J. J. Mandemakers (forthcoming). Is the association between education and fertility postponement causal? The role of family background factors, Demography 
felix-tropf.com

\subsection{Introduction}

A large body of literature from the USA and Europe documents a positive relationship between higher educational attainment and later age at first birth of women (e. g. Gustafsson, Kenjoh, \& Wetzels, 2002; Lappegård \& Rønsen, 2005; Martin, 2000). It has been argued that this relationship is causal and that it can account for the rise in the mean age at first birth during the educational expansion of the second half of the 20 $0^{\text {th }}$ century in Western countries (Balbo et al., 2013; Ní Bhrolcháin \& Beaujouan, 2012). However, research also casts doubt on the idea of a causal effect of education on the age at first birth, suggesting that family background characteristics (social and/or genetic factors) cause spurious associations between educational attainment and fertility timing of women (Neiss et al., 2002; Rodgers et al., 2008). Social stratification research indicates that there is considerable similarity between parents-children and siblings in education and socio-economic attainment (Branigan, McCallum, \& Freese, 2013; van Doorn et al., 2011) and in fertility behavior (Murphy, 1999; Rijken \& Liefbroer, 2009). The similarity/intergenerational transmission may be due to parental socio-economic resources and/or socialization processes (Nisén \& Myrskylä, 2014; Rijken \& Liefbroer, 2009), but also to shared genetic dispositions, as there is sufficient evidence for a genetic component for both outcomes (Branigan et al., 2013; Mills \& Tropf, 2016).

Our study contributes to the existing literature addressing the following three questions: first, does education indeed have a causal effect on age at first birth? Second, to what extent can the postponement of age at first birth during the second half of the twentieth century be explained by the simultaneous educational expansion? Third, to what extent are (social) environmental family background and/or genetic factors responsible for the observed relationship between education and age at first birth?

To answer these questions, we present within(-identical) twin models and engage in biometric modeling of the link between education and age at first birth in order to disentangle a possible causal relationship from genetic and environmental confounders in a large sample of twins from the UK. Next to quasi-experimental study designs, within-twin designs offer an approach to causality. Quasiexperimental designs (McCrary \& Royer, 2011; Skirbekk, Kohler, \& Prskawetz, 2006) 
use exogenous factors, such as changes in compulsory school laws, which influence education but are supposed to be independent from fertility to investigate the causal effect of education on fertility. The within-twin approach, in contrast, controls for all factors shared amongst siblings, including genetic material, by using identical twins as a natural experiment (Kohler et al., 2011). The experiment is that the twins differ in levels of education, but neither in their genetic make-up nor their family environment. To the extent that the family background (social and/or genetic factors) is a common cause for education and age at first birth, standard regression models result in biased estimates, while within-(identical) twin estimates remain unbiased (Amin et al., 2015).

The within-identical twin approach is of particular interest for three reasons. First, as mentioned before, we expect that most of the unobserved factors important for education and fertility timing are related to the family of origin, which can be controlled for in twin studies (Branigan et al., 2013; Mills \& Tropf, 2016). Second, the within-identical twin design can use variation across all levels of education, whereas for example quasi-experimental on school-law-change studies mostly rely on variation on a particular (mostly lower) level of education (see also Amin et al., 2015). Third, comparing fraternal and identical twins allows a quantification of the contribution of genetic and environmental influences for a specific trait, as in a classic-twin and behavior genetics modeling (Rodgers et al., 2007; Rodgers, Kohler, et al., 2001). Kohler and colleagues (2011) recently developed a new model, which formally integrates the two approaches: the within-identical twin regression model and the behavior genetics model. This so-called ACE-beta model represents a parsimonious solution to identify causality in a bivariate association and simultaneously evaluate the importance of genetics and shared environmental influences from the family.

Demographic research should strive to connect the individual and population level (Billari, 2015). We therefore combined our findings at the micro-level with nationally representative data from the Office for National Statistics (ONS) to evaluate whether the causal effect of education explained age at first birth trends at the population level. This was an important goal, not only in order to understand past fertility trends, but also to assess the potential to anticipate future fertility development based on changes in educational level - as education has gained 
importance in fertility forecasting (Lutz, Butz, \& Samir, 2014). Furthermore, in the $\mathrm{UK}$, the trend in age at first birth was U-shaped during the mid-century in the UK (Hobcraft, 1996), whereas educational expansion increased steadily (Oreopoulos, 2006). These differential trends motivated a closer inspection of the relationship in different birth cohorts. We therefore also present analyses separately for birth cohorts born before and after the Second World War.

The current study builds upon and extends previous research in a number of ways. First, we applied the new ACE-beta model to study the link between education and age at first birth in a large sample of 2,752 twins from the TwinsUK. This is the largest adult twin register in the United Kingdom (Moayyeri et al., 2013) and it offers a larger sample size than was available to earlier research.

Second, previous investigations of the causal link between education and age at first birth in family designs showed mixed results, partly confirming a causal relationship (Amin \& Behrman, 2014; Nisén et al., 2013) and partly not (Neiss et al., 2002; Rodgers et al., 2008). However, these studies also engaged in different analytical strategies, applying within-identical twin models (Amin \& Behrman, 2014), standard behavior genetics models (Nisén et al., 2013) and complex structural equation models (SEM) (Neiss et al., 2002; Rodgers et al., 2008). To contribute to this puzzle we present within-identical twin models as well as the ACE-beta model that simultaneously estimates the causal effect and quantifies family environmental and genetic influences on the education-fertility relationship in a parsimonious SEM (Kohler et al., 2011).

Third, the UK is a particularly interesting case to study. Comparative studies within Europe suggest that the effect of education on age at first birth is relatively strong in the UK (Gustafsson et al., 2002; Rendall et al., 2005). A recent investigation by Ní Bhrolcháin \& Beaujouan (2012) showed that in the UK between 1980-1999, 57 percent of the postponement of the mean age at first birth could be attributed to longer educational enrolment, while the remainder was due to additional, posteducational postponement effects - if we endorse the said causal relationship between education and age at first birth.

In the following, we first discuss the previous (bio-)demographic literature, continue by introducing the TwinsUK dataset, the within-twin method and the new ACE-beta model. We subsequently present our results and discuss our findings. 


\subsection{Background}

\subsubsection{Education and fertility timing}

The mean age at first birth of women steeply increased by up to 4-5 years during the second half of the twentieth century all over Europe and the US, and was accompanied by an overall increase in educational attainment (Mills et al., 2011). In the UK, between 1980 and 2000, for example, the average age at first birth as well as age of leaving full-time education increased by 1.4 years (Ní Bhrolcháin \& Beaujouan, 2012). Joshi (2002) showed by comparing three UK birth cohort studies $(1946,1958,1970)$ that the percentage of women who were already mothers by age 26 dropped from 81 percent for those born in 1946 to 30 percent for the 1970 cohort. In all three birth cohorts, women with tertiary educational qualification were about half as likely to be mothers than women with no educational qualifications. In the most recent 1970 cohort, only 1 in 10 higher educated women were mothers at age 26, compared to 6 in 10 for women without qualifications.

A number of causal mechanisms have been put forward to explain the association between educational level and fertility timing (for an overview see Balbo et al., 2013; Mills et al., 2011). First, being enrolled in education itself may lead to postponement of childbearing, as it is difficult to combine the student role with the mother role, since both entail time intensive tasks. Women might delay childbearing due to high costs of children and few resources during the time of their studies or social norms might discourage parenting before the end of education (Hoem, 2000; Lappegård \& Rønsen, 2005). Second, education may increase people's aspirations and ability to pursue a career. So, women might further postpone childbearing until they are well established in their careers - also implying higher opportunity costs of the transition to parenthood given their larger human capital (e. g. Liefbroer \& Corijn, 1999). Furthermore, education might also change values and orientations towards more individualistic lifestyles that seek fulfillment in life without children (Lesthaeghe, 1995; van de Kaa, 1987). Therefore, both educational enrolment and attainment may lead to postponing childbearing (and reducing fertility). Reverse 
causality may play a role too because an early age at first birth may disrupt further education, although this is presumably mainly the case for unwanted teenage childbearing (Nisén \& Myrskylä, 2014).

Reasoning along these lines and noting the often observed micro-level association led to the hypothesis that educational expansion in the UK explains the postponement of age at first birth (Ní Bhrolcháin \& Beaujouan, 2012). However, such an interpretation is not straightforward for at least three reasons. First, the observed macro-level association between education and age at first birth changed across the past century; the simultaneous rise in age when leaving education and age at first birth only occurred after WWII. Second, in addition to the overall rise in education, there are a number of competing explanations for the rise in (female) age at first birth in developed countries. Third, as touched upon before, the relationship between education and age at first birth may be partly spurious. We now discuss these issues in more detail.

\subsubsection{Changing macro trends}

Comparative studies within Europe indicate that there is variability in the association between education and age at first birth (Gustafsson et al., 2002; Rendall et al., 2005). More specifically, in the UK the association between education and age at first birth on the population level changed across the past century. Age at first birth decreased in the period after the Second World War, and there was an accompanying steep increase in fertility - the so-called baby boom. At the same time educational attainment increased. The UK's Education Acts from 1918 and 1944 lifted the school leaving age from 12 to 14 and later from age 14 to 15 . As shown by Oreopoulos (2006), within three years during the period of 1945-1947 the fraction of 14-year-olds leaving schools fell from about 57 percent to less than 10 percent. Only later that century, since the 1960s, has age at first birth increased - the so called baby-bust. Education further expanded, and trends in age when leaving education and age at first birth ran parallel. Given the reversing trends throughout the century, we hypothesize that a strict causal logic that longer educational enrolment leads to later age at first birth may not apply. 


\subsubsection{Fertility postponement: Alternative explanations}

Several alternative explanations - mainly related to the introduction of the 'pill'- have been put forward to explain fertility postponement in developed countries during the $20^{\text {th }}$ century. It has been shown that rising female labor force participation (e. g. Rindfuss, Guilkey, Morgan, Kravdal, \& Guzzo, 2007), ideational shifts in norms and values in sexual behavior and family planning of the second demographic transition (Lesthaeghe, 1995; van de Kaa, 1987) and increasing economic uncertainty (Andersson, 2000) are associated with an increasing age at first birth and might (partly) account for the postponement. The introduction of the 'pill' as an effective contraception is seen as an important trigger of these mechanisms.

In the UK, especially changes in family norms and values, as well as economic uncertainty, and the introduction of the pill provide alternative explanations for fertility postponement during the last 50 years, which may operate together with or independent of the educational expansion (Hobcraft, 1996; Murphy, 1993). To evaluate to what extent the educational expansion can be held responsible for fertility postponement and avoid ecological fallacy, we assess the causal effect of education on age at first birth on the individual level and use these estimates for predictions at the population level on nationally representative and independently collected data.

\subsubsection{Critique of education and fertility link}

The association between education and age at first birth might be in part spurious because unobservable factors influencing age at first birth may also be related to education. In particular, the family background may play a pivotal role as a socializing agent, a source of resources and support, and for transmitting genes. Studies have repeatedly demonstrated that education (Branigan et al., 2013) and fertility (Mills \& Tropf, 2016) are associated with both the family environment and genetic factors. Therefore the family environment and genetic factors may (partly) explain the observed association between education and age at first birth.

Family background, for example, the socio-economic status of the parents, defines the resources and opportunities to remain in school longer and to financially 
compensate for children. The status of parents can shape consumption and status aspirations of children who aim for higher education and social status in advance of family formation (Thornton, 1980). More generally, the socialized striving for autonomy might lower ambitions to build a family and increase investments in education and a career (Rijken \& Liefbroer, 2009; Scott, 2004). Biological predispositions shared among family members and transmitted through genes can influence career and family trajectories. The timing of first attempts to get pregnant measured in retrospective interview is linked to one's genetic make-up (Rodgers, Kohler, et al., 2001). It is also established that genes influence educational attainment (Branigan et al., 2013; Rietveld, Medland, et al., 2013), and fertility timing (Nisén et al., 2013; Tropf, Barban, et al., 2015). However, the question remains to what extent genetic effects for both outcomes are shared. In the following, we discuss previous studies that investigated the influence of (bio-)social family factors on education and fertility.

\subsubsection{Biometric approaches for the education and age at first birth link}

To our knowledge, four previous studies have considered both genetic and (socially) environmental family influences on the relationship between education and age at first birth. The first two investigations focus on the role of education as a possible mediator between cognitive ability and age at first birth. One used 813 pairs of relatives from the NLSY born between 1958-65 (Neiss et al., 2002) and the second 621 Danish twins pairs born between 1931-52 (Rodgers et al., 2008). They extend the classic twin model by introducing cognitive ability as preceding education in the causal chain at the cost of additional assumptions on shared genetic and environmental effects across traits. Both studies find that the observed mediating link of education turns non-significant after controlling for genetic and environmental influences from within the family. These two studies did not find genetic influences on age at first birth and thus no genetic correlation between both outcomes, which contrasts the general pattern (Mills \& Tropf, 2016). They conclude that education may not directly delay childbearing, but individual differences which lead to higher cognitive ability and higher education inhibit fertility, and these differences arise between, not within families. 
The third study estimated bivariate biometric models, which decomposed the (co)variance in education and age at first birth into latent genetic and environmental factors in a sample of 4,228 Finnish twins born in 1950-1957 (Nisén et al., 2013). For women they report that genetic, shared environmental factors of the twins as well as environmental factors unique to an individual independently explain part of the observed covariance between education and age at first birth. Influences unique to an individual can be interpreted as a causal effect (D'Onofrio, Lahey, Turkheimer, \& Lichtenstein, 2013), but might also result from third factors influencing both outcomes at the same time such as the partner (Kohler \& Rodgers, 2003).

Finally, Amin et al. (2014) used a within-identical twin design on a sample of 628 identical twins from the Minnesota Twin Registry. They find that one year of additional education leads to fertility postponement of around one year. They furthermore do not find evidence for family backgrounds effects on the association between education and age at first birth, that is, results in standard OLS regression models are nearly identical to the within-twin models.

In the current study, we first focus on the causal relationship between education and age at first birth and apply within-twin regression models. We present a within-identical twin estimate in a different context than Amin et al (2015), and for a larger sample. Second, we use our findings to project the age at first birth trend on the population level and contrast the projection with the actual age at first birth trend. Third, we extend these models according to Kohler et al. (2011) by integrating the bivariate twin model. Compared to previous mediation models (Neiss et al., 2002; Rodgers et al., 2008), the ACE-beta model needs fewer identifying assumptions and all paths we introduce to the model are identified and can be estimated. 
felix-tropf.com

\subsection{Data \& method}

\subsubsection{Data}

We used information on twins of the TwinsUK registry. TwinsUK was originally established at the St. Thomas Hospital London in 1992 and gathered information on the life course of identical or monozygotic (MZ) and fraternal or dizygotic (DZ) twins (Moayyeri et al., 2013). Zygosity was established using standardized questions and confirmed by DNA genotyping in uncertain cases. Currently it contains information on about 12,000 individuals. We limited the analysis to same sex female individuals in complete twin pairs because the TwinsUK contains few male twins $(<15 \%)$ so that a comparable analysis for men was not feasible. We furthermore excluded women younger than 40 years old at last moment of observation to avoid an over-representation of young mothers and limit rightcensoring of women who did not have children at the time of last observation. Valid information on zygosity, fertility and education was available for 3,856 women in 1,928 twin pairs. The sample is further reduced due to right-censoring of one or both twins in a twin pair to 2,752 women in 1,376 twin pairs. Note that for robustness checks, we also applied Cox regression models that include childless women (please see the SI 5-1, SI 5-2 and SI 5-3).

In order to compare the TwinsUK sample to a representative sample and to describe trends we used two additional sources from the ONS. For education, we used data from the General Household Survey (GHS) rounds from 2000 to 2006 $(\mathrm{N}=35,435$, birth cohorts 1931-1970). The GHS is an annual continuous survey of the population in private households in Great Britain. To describe age at first birth we used estimates from the Office for National Statistics (Office National Statistics, 2013) because historical GHS fertility measures were partly limited to married individuals.

\section{Age at first birth}

The measure for age at first birth was based on information from two questionnaires in the TwinsUK. First, the 'Main Questionnaire', which was administered between 1995 and 2001, contains an inventory of the years of birth of 
up to ten children. Second, age at first birth was assessed directly with the question, "How old were you when you had your first live birth?", taken from the 2004 questionnaire. In case individuals participated in multiple waves, we used the earliest reported age.

\section{Education}

Education was measured as the age at leaving full-time education. This was assessed directly with the question: "At what age did you leave full-time education?" Most previous work has measured education in categories and imputed the corresponding years of schooling (e. g. Amin \& Behrman, 2014; Rodgers et al., 2008). In the UK, it is difficult to order educational categories vertically due to differences in educational systems and migration across countries within the UK, especially because qualifications are so numerous, and because their names and content changes often (Jenkins \& Sabates, 2007). Therefore, both TwinsUK and the GHS provide the age when leaving full-time education as a valid and reliable measure across data sources.

\subsubsection{Analysis}

The analysis proceeds in two steps. First, we present ordinary least square regression (OLS) and subsequently within-twin models (fixed effect models) to estimate the causal effect of education on age at first birth (AFB). Although withintwin models have a long history in economics (Rosenzweig \& Wolpin, 1980) and psychology/ behavioral genetics (D'Onofrio et al., 2013; Neale \& Cardon, 1992), there are only some applications of within-twin models in demography (Amin \& Behrman, 2014). A comparison of the OLS and the within-twin approach indicates to what extent family background leads to a spurious association between education and age at first birth. In a second step, we estimated the ACE-beta model to investigate to what extent genetic and/or environmental effects lead to a spurious association between education and age at first birth. 
felix-tropf.com

\section{OLS and fixed effects models}

The OLS-models include birth year and birth year squared of a twin (i) nested in a twin pair ( $\mathrm{j}$ ) to allow for a curvilinear trend in fertility timing across birth cohorts. Furthermore, we control for zygosity measured as the expected genetic relatedness between DZ twins (0.5) and MZ twins (1) and estimate robust standard errors to correct for the dependency structure of the twins (equation 1). We are interested in the effect of education $\left(\boldsymbol{\beta}_{\mathbf{1}}\right)$.

afb $_{i j}=\beta_{0}+\beta_{1} * e d u_{i j}+\beta_{2} *$ birth year $_{j}+\beta_{3} *$ birth year $_{j}^{2}+\beta_{4} *$ zygosity $_{j}+\varepsilon_{i j}(1)$

The OLS model gives a 'naive' estimation of the association between education and age at first birth. As detailed elsewhere (Amin, Behrman, \& Spector, 2013; Kohler et al., 2011), standard OLS ignores at least three additional sources of variation; first the shared environment in a family $\mathrm{j}$ specific for fertility $\left(\boldsymbol{C}_{\boldsymbol{j}}\right)$ such as family norms, second the individual additive genetic endowment $\left(\boldsymbol{A}_{\boldsymbol{i j}}\right)$, and third the unmeasured unique environment $\left(\boldsymbol{E}_{\boldsymbol{i j}}\right)$, which includes measurement error and individual specific effects for fertility, such as the influence of partner characteristics. These effects might impact the estimation of $\boldsymbol{\beta}_{\mathbf{1}}$ if they are correlated with education.

The within-twin models include fixed effects per family, which capture all (observed and unobserved) factors shared among the twins. We can discriminate between fraternal or dizygotic (DZ) and identical or monozygotic (MZ) twins. DZ twins share on average 50 percent of their segregating genetic material, MZ twins are genetically identical, so that a DZ-fixed effects model controls for half of all additive genetic effects (similar to a sibling fixed effects model) and the MZ-fixed effects model controls for all genetic effects. In other words, the MZ-fixed effects model controls for all factors shared amongst siblings, including birth year, zygosity, but also common (family) environment $\left(C_{j}\right)$ and genetic effects so that $\Delta\left(C_{i j}\right)=$ 0 and $\Delta\left(A_{i j}\right)=0$. The equation for the MZ-fixed effects is:

$$
\Delta(a f b)_{i j}^{m z}=\beta_{1} * \Delta(e d u)_{i j}^{m z}+\Delta \varepsilon_{i j}^{m z}
$$


We applied both the OLS regression and the twin-fixed effects models to the pooled sample of twins, as well as for DZ and MZ twins separately. The comparison, particularly of the $\mathrm{DZ}$ and $\mathrm{MZ}$ models gives the first insights as to whether differences between the 'naive' OLS estimates and the fixed effects estimates are due to shared environmental and/or genetic factors. If genetic effects are important, we expect a stronger reduction in the effect of education for MZ-twins than for DZtwins. The remaining link between education and age at first birth can be interpreted as causal. The effect is consistent if unique environmental influences important for education are independent of age at first birth (and vice versa) (Kohler et al., 2011).

\section{Bivariate Genetic Modelling}

Twin studies are no longer uncommon in social science (Branigan et al., 2013; Freese, 2008; Kohler et al., 2011, 1999; W B Miller et al., 2010; Mills \& Tropf, 2016; Neiss et al., 2002; Nisén et al., 2013; Rodgers, Kohler, et al., 2001; Rodgers et al., 2008; Tropf, Barban, et al., 2015). Briefly: Twin studies are based on the comparison of MZ and DZ twins in order to quantify genetic and environmental influences. The degree to which MZ twins are more similar than DZ twins is assumed to reflect only genetic influences. Twin models are a typically SEMs that decompose the observed variance into three components: (a) additive genetic effects resulting from the sum of genetic effects from the whole genome (A), (c) environmental effects resulting from environmental influences shared between twins of a pair $(C)$ and $(e)$ non-shared environmental effects resulting from the unique environment of an individual (E) (which includes measurement error).

Three assumptions of the twin model need to be briefly addressed: The first is that $\mathrm{MZ}$ and DZ twins share their environment to the same extent (the equalenvironment assumption (EEA)). This assumption has repeatedly been criticized (e.g., Horwitz et al. 2003), however, empirical evidence supports the validity of this assumption (Conley et al. 2013), including fertility studies (Felson, 2014). Second, it is assumed that there is no assortative mating within the population with respect to the outcome of interest. A violation of this assumption (Domingue and Fletcher 2014) would result in an underestimate of genetic influences. The third assumption 
felix-tropf.com

is that there are no non-additive genetic effects (gene-gene, and gene-environment interaction effects).

Following the same logic as in classic twin studies, it is possible to estimate the extent to which genetic and/or environmental factors are important for the covariance between two different outcomes. If education of twin 1 correlates with age at first birth of twin 2, then part of the covariance runs in families. If this correlation is higher amongst MZ-twin pairs than DZ-twin pairs, this indicates shared genetic effects for both outcomes. We estimate the ACE-beta model (Kohler et al., 2011), which represents an extension of a bivariate Cholesky decomposition (for details see Loehlin, 1996; Rodgers et al., 2007). The bivariate Cholesky decomposition estimates two equations, one for the first variable, in our case education (eq. 3), and one for the second variable, in our case age at first birth. Education of twin (i) is a function of:

$$
e d u_{i}=a_{e d u, e d u} *\left(A_{i j}^{e d u}\right)+c_{e d u, e d u} *\left(C_{j}^{e d u}\right)+e_{e d u, e d u} *\left(E_{i j}^{e d u}\right)
$$

Indexes indicate whether the effects are on education (edu), age at first birth (afb) or shared for both. Lower-indexes indicate whether the effects are common to both twins ( $\mathrm{j}$ ) or different (ij). The variance in education is therefore decomposed into additive genetic effects $\left(a_{e d u, e d u}\right)$, shared environmental effects $\left(c_{\text {edu,edu }}\right)$ and residual variance/measurement error $\left(e_{e d u, e d u}\right)$.

Investigating the association between education and AFB, we formulate a second equation, which decomposes the variance in AFB into parts that are common with the genetic variance components of education (for example $a_{e d u, a f b} *\left(A_{i j}^{e d u}\right)$ and the shared environmental component $\left(c_{\text {edu,afb }} *\left(C_{j}^{e d u}\right)\right)$ and parts that are unique to AFB - caused by $A^{a f b}, C^{a f b}$ and $E^{a f b}$. In contrast to the classic bivariate Cholesky model, the ACE-beta model assumes that unique environmental sources of variance/measurement error for education $\left(E_{i j}^{e d}\right)$ are uncorrelated with $\mathrm{AFB}$ $\left(e_{\text {edafb }}=0\right)$. Instead, the link between education and AFB is expressed by the causal link represented in a fixed-effects regression only within MZ-twin pairs $\beta_{1} *$ $\Delta(E d)_{i j}^{m z}$ (eq. 4$)$. 


$$
\begin{aligned}
& a f b_{i j}=\beta_{1} * \Delta(e d u)_{i j}^{m z}+a_{e d u, a f b} *\left(A_{i j}^{e d u}\right)+c_{e d u, a f b} *\left(C_{j}^{e d u}\right)+ \\
& +a_{a f b, a f b} *\left(A_{i j}^{a f b}\right)+c_{a f b, a f b} *\left(C_{j}^{a f b}\right)+e_{a f b, a f b} *\left(E_{i j}^{a f b}\right)
\end{aligned}
$$

In the main text, we visualize the standardized estimates in the form of a correlated factors model (Loehlin, 1996; Neale \& Cardon, 1992) to facilitate interpretation. We present all results from these models and the standardization of the estimates in the SI. The genetic contribution to education and AFB is typically expressed as narrowsense heritability $\left(h^{2}\right)$, which is the proportion of variance due to additive genetic effects. In a parallel way we can compute the variance component for shared $(C)$ and unique environmental influences/measurement error (E). Importantly, we give an estimate to what extent genetic and shared environmental influences important for education and age at first birth correlate and explain the observed correlation between education and age at first birth.

We used Stata 12 to estimate the OLS and fixed effect models and we graciously received the OpenMX R-function to estimate the 'ACE-beta' model from the developers Kohler et al. (2011).

\section{Robustness}

The main analysis presented in this paper included only twin pairs for whom both twins had a child because the bivariate biometric models cannot deal with nonlinear outcomes. We tested the robustness of our results by including right-censored observations using Cox-regression models. We present Cox regression models with and without stratification by family to replicate the OLS and within-twin models for the full sample (Allison \& Christakis, 2006). Please see the SI for details.

\subsection{Results}

\subsubsection{Descriptive findings}

Table 5-1 shows the descriptive statistics of the variables of interest separately for DZ and MZ twins. The twins in the sample were born on average just after WWII, 
mean age at first birth was almost 26 and the mean age at leaving education was about 17 years for both kinds of twins. Most importantly for the biometric models, there were only minor differences in average or standard deviation of the outcomes of interest between $\mathrm{DZ}$ and $\mathrm{MZ}$ twins. It should also be noted that only 27 individuals $(\sim 1 \%)$ had their first birth before leaving education and only $74(\sim 2.7 \%)$ until one year after leaving education so that the temporal succession suggests that education influences age at first birth and not vice versa.

There are two main concerns about the within-twin approaches: first that variation of the independent variables within twin pairs is low and largely due to measurement error, and second that twin data are not representative. To address these concerns, first, Table 5-1 shows the mean absolute differences in education within twin pairs. These differences were substantial with 1.33 for DZ and 0.88 for $M Z$ twins, which suggests that sufficient variation remained even within $M Z$ twin pairs. The smaller differences for MZ than DZ twins suggests a genetic component underlying education. Second, TwinsUK is considered to be representative for the singleton population in the UK (Andrew et al., 2001; Moayyeri et al., 2013). Figure 51 shows the smoothed trends in age when leaving education and age at first birth for the representative ONS/GHS data (top panel) and the TwinsUK sample (lower panel). The general level and trends for the TwinsUK sample were similar to that of the ONS/GHS data, which increases our confidence in using the TwinsUK sample. However, it is to be noted that the mean age at first birth in the TwinsUK sample (25.83) was about a year earlier than in the ONS data (27.00). There was no difference in age at leaving education between the twinsUK (16.97) and GHS (16.92) estimates.

Moreover, it is clear from Figure 5-1 that the mean age of leaving education rose steadily throughout the $20^{\text {in }}$ century, whereas the mean age at first birth followed a U-shaped pattern in the UK. Most previous research focused on trends in both outcomes during the second half of the $20^{\text {th }}$ century, concluding that the rise in age at finishing education leads to a postponement of childbearing. However, the figure reveals a discontinuity in the association, which challenges the idea of a causal relationship between education and age at first birth. 


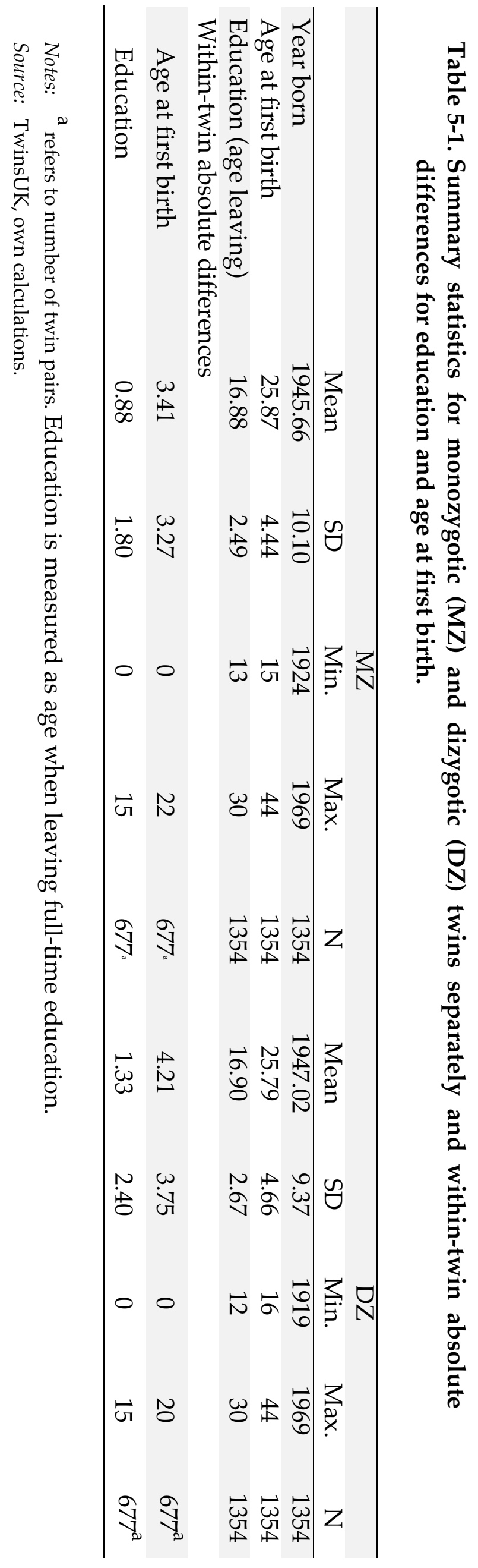



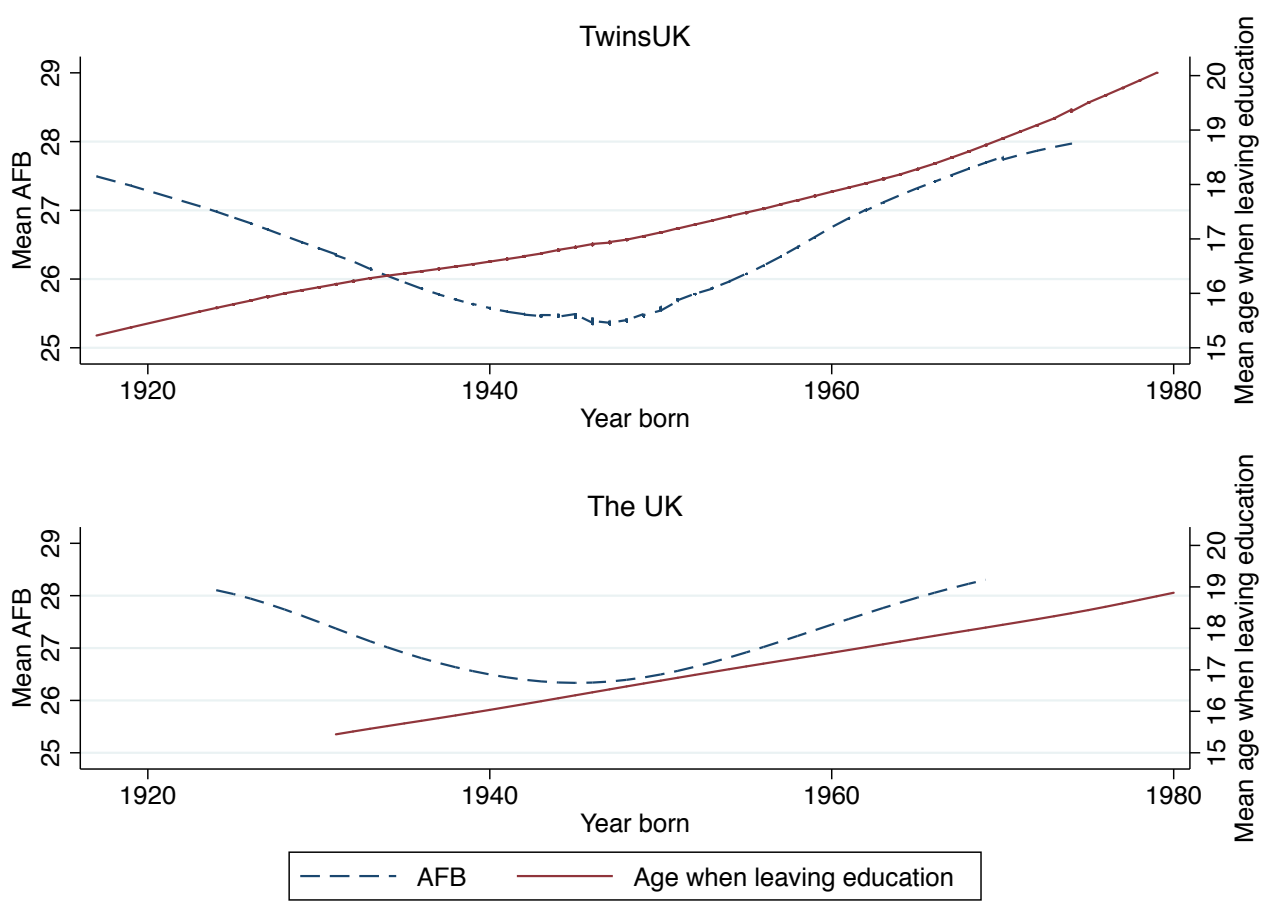

Figure 5-1 Trends in mean age at first birth and mean age when leaving full-time education in the TwinsUK sample and national representative data of the United Kingdom

Notes: $\mathrm{AFB}=$ age at first birth; The UK = estimates from large, nationally representative data from the Office for National Statistics

Source: TwinsUK and data from the National Office for Statistics (for details see methods section).

\subsubsection{The causal effect of education on age at first birth}

Our first research question asks: Is there a causal effect of education on age at first birth? Model 1 in Table 5-2 estimates the well-established naive OLS estimate of the effect of education on age at first birth at .44 (standard error $(\mathrm{SE})=0.04$ ) in the present sample. This means that women who stayed one year longer in education had their first childbirth about half a year later on average. The effect holds independent of cohort effects as we controlled for birth year and its square. Model 3 and model 5 repeat the same model but now for subsets of MZ and DZ twins separately. The estimates of MZ and DZ twins were nearly identical. 
Now we turn to the twin-fixed effects models to disentangle the causal effect from potential family confounders. Model 2 in Table 5-2 shows the pooled twinfixed effects approach, which controls for all shared environmental factors among siblings and partly their genes. The effect of education on age at first birth fell to .11 $(\mathrm{SE}=0.06)$, but remained significantly different from 0 at the 5 percent level in a onesided test. Model 4 depicts the MZ-fixed effects estimate $(0.12, \mathrm{SE}=0.09)$ that controlled for all factors that vary between twins including all shared environmental and genetic differences, as MZ twins are genetically identical. The twin-fixed effects estimate for DZ twins is depicted in model $6(0.11, \mathrm{SE}=0.08)$. This estimate controlled for all factors that vary at the family level; genetic differences among DZ twins were only partly controlled for. The pooled, MZ-fixed effects, and DZ-fixed effects estimates were all similar in size (around .11), but the the two twin-fixed effects analyses by zygosity were not significantly different from 0 . This was probably due to reduced power of the twin-fixed effects approach. This suggests a reduction in the causal effect of education on age at first birth with about three quarters $(1-.12 / .44)$ to about 1.4 months $\left(.12^{\star} 12=1.44\right)$. These findings suggest that a large part of the education effect on age at first birth can be attributed to family background factors (genetic and environmental).

In order to check the robustness of these findings when also including twin pairs where one or both women did not have children at the time of last observation and those younger than 40, SI 5-3 provides the results from the (stratified) Cox regression models $(N=4,398)$. Results followed the same pattern as the regression models except that the reduction in the estimated effect of education for the withintwin analyses appeared to be less strong. The probability of having a child decreased with each additional year of education by $\sim 10$ percent and this fell to $\sim 3$ to 4 percent in the stratified Cox regression models. 


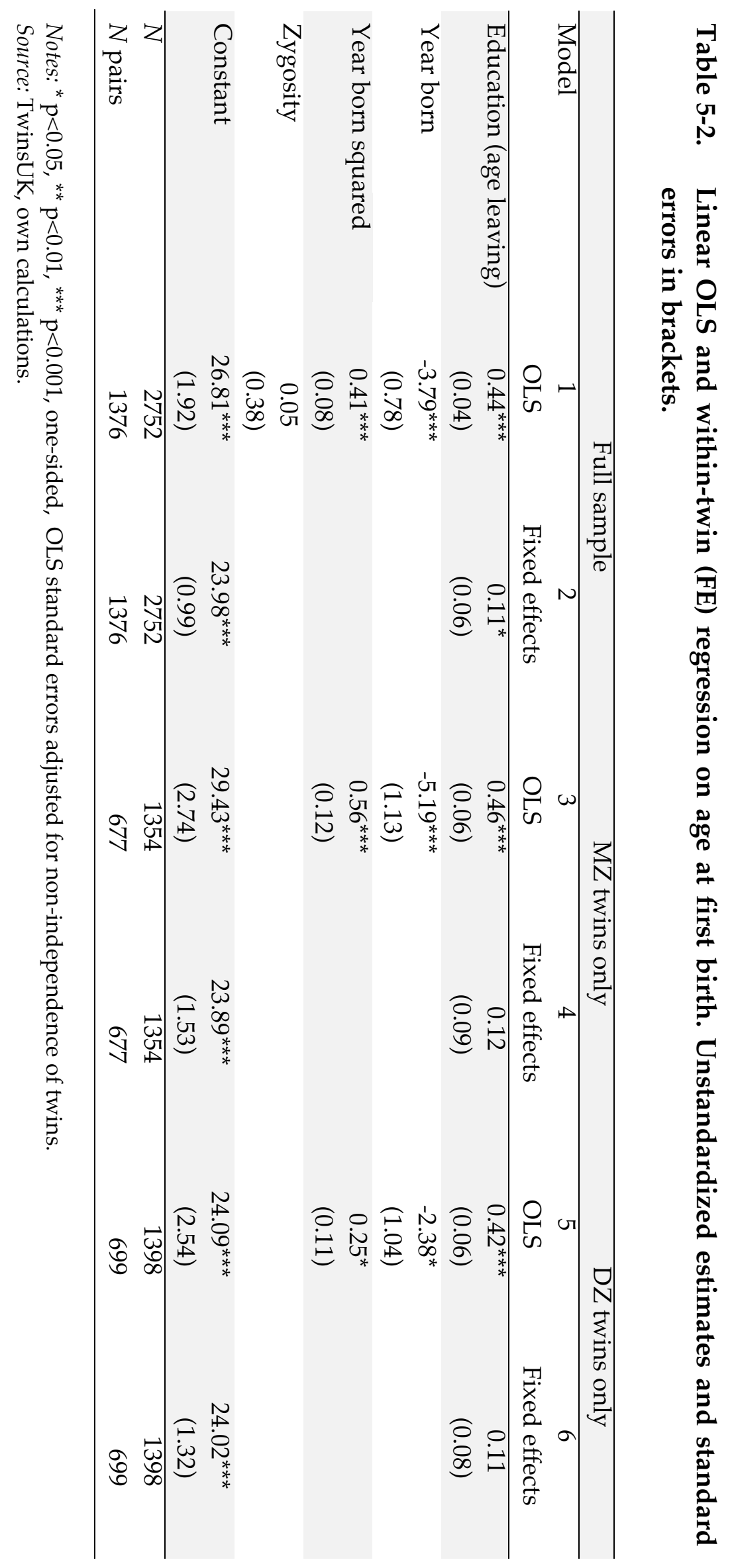


Our second research question asked: to what extent can the educational expansion during the second half of the twentieth century explain the simultaneous overall postponement in age at first birth? Figure 5-2 depicts the simultaneous rise in age of leaving education (dashed line) and age at first birth (solid line) for cohorts born between 1944-1969. These cohorts started childbearing in time periods since the 1960s and therefore can be considered the main drivers of the fertility postponement. Age at first birth in 1969 in the UK (28.15) was about 2.4 years later than in 1944 (25.75). Figure 5-2 furthermore shows the simultaneous rise in age of leaving fulltime education of around 1.3 years across these birth cohorts. A straightforward macro-explanation might consequently conclude that 54 percent $(=1.3 / 2.4)$ of the 2.4 year of fertility postponement can be attributed to a rise in educational enrolment. However, as shown in the regression models, for each year of additional education, individuals postpone $\sim 5.3$ months in the OLS model and $\sim 1.4$ months in the withintwin models. Note that we use a reduced MZ-estimate of $1.2\left(.10^{*} 12=1.20\right)$ months since we restrict the analysis to MZ-twins born after 1944 (not listed). Across birth cohort born between 1944-1969 only between 24 percent $\left(=1.3^{*} .44 / 24\right.$; OLS models) and 5.4 percent $\left(=1.3^{*} 0.10 / 2.4\right)$ of the observed postponement in age at first birth can be directly related to the educational expansion. Figure 5-2 shows the predicted average age at first birth based on the OLS (short dash-dot-dot) and MZ twin-fixed effects results (dash-dot), as well as the explained (green) and unexplained (red) age at first birth trend across the second half of $20^{\text {th }}$ century and in yellow the difference between OLS and MZ twin-fixed effects model. The OLS specification can be considered as the upper bound of the explained trend in AFB by education, and the MZ-fixed effects model the lower bound. to MZ-twins born after 1944 (not listed).

The estimates in Table 5-2 are based on birth cohorts born between 1919-1969. As shown in Figure 5-1, the correlation of the trends in education and age at first birth reversed on the population level and it is possible that the same applies to the individual level. Therefore, the causal effect of education might have become stronger in more recent cohorts. We additionally estimated models that allow for different educational effects for birth cohorts born before and after 1944 (the turning point in age at first birth trend) to investigate this issue (see SI 5-4). However, we do not find such differences. 


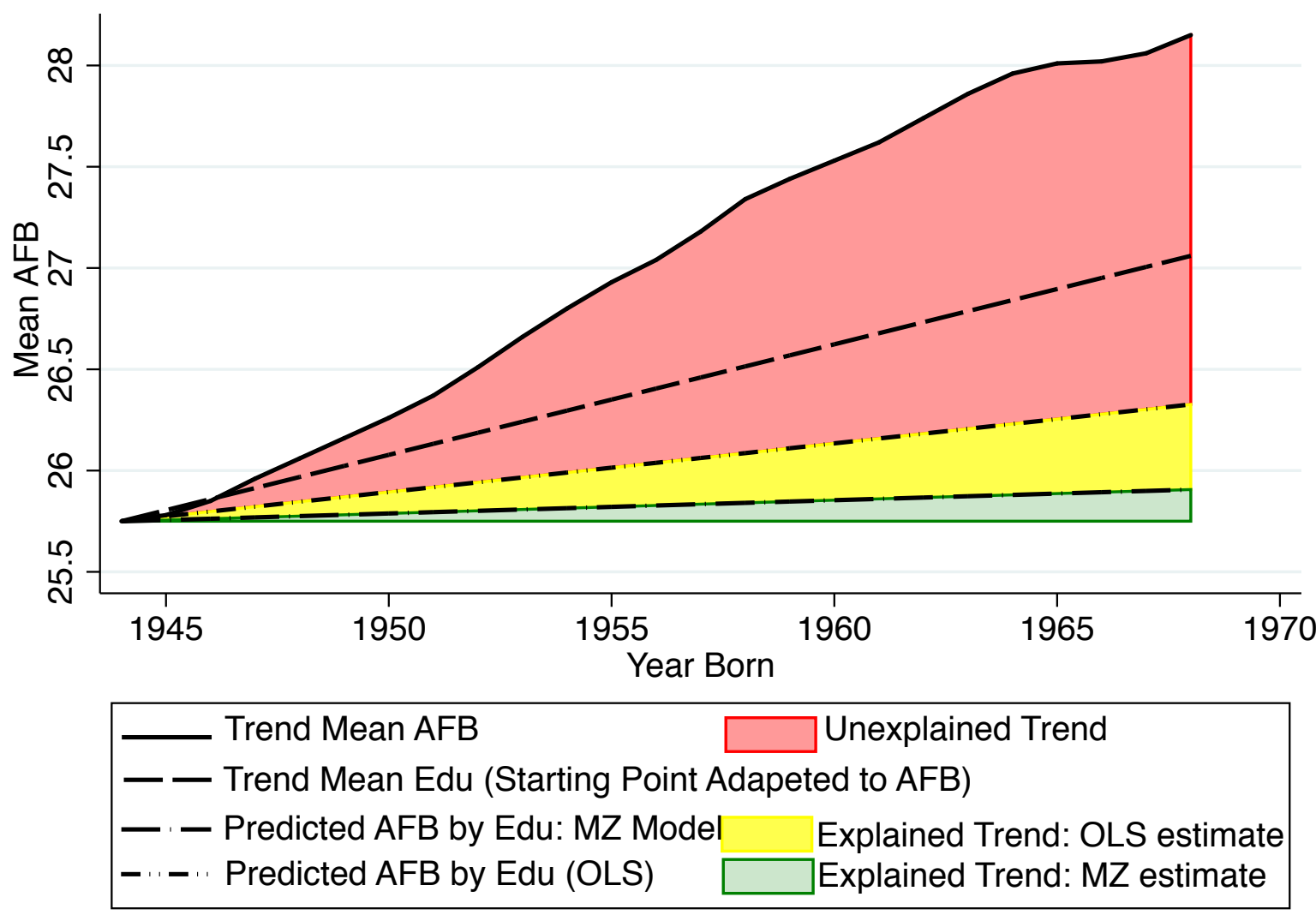

Figure 5-2. Can educational expansion explain postponement of childbearing since 1945? The graph depicts the observed trends in mean age at first birth and education (age at leaving) for successive birth cohorts, and the predicted trend in age at first birth by education using the OLS and MZ estimates

Notes: The trend for educational level was rescaled to that of age at first birth. Predictions were based on estimates from Model 2, Table 5-2, and data from Table 5-1.

Source: General trends in education were derived from British General Household Surveys (20002006) and age at first birth from the Office for National Statistics, Cohort fertility, Table 2. (Office National Statistics, 2013) 
5.4.3 The role of genes and the environment as family background factors

The reduction of the estimate of the causal effect of education on age at first birth in OLS versus MZ twin-fixed effects models leaves us with the third research question: To what extent is this due to unobserved shared environmental influences and/or unobserved genetic endowments that are common to both outcomes. In order to answer this question, we present the ACE-beta model, which simultaneously estimates the direct causal link between education and age at first birth based on the MZ twin-fixed effects estimator and the genetic and shared environmental contribution. Figure 5-3 presents the correlated factor model (please see SI 5-5 and 5-6 for the untransformed SEM estimates and the transformation).

The estimates are well in line with previous studies (Branigan et al., 2013; Mills \& Tropf, 2016). The heritability of education was 0.46 , meaning that 46 percent of the variance in education was associated with additive genetic differences. The shared environmental influences account also for 23 percent of the variance, and the unique environment, which includes measurement error, for 31 percent. The heritability of age at first birth is estimated to explain 32 percent of the variance in age at first birth. Shared environmental effects were smaller (0.12) but significant and unique environmental effects/measurement error, made up the largest part (0.56).

All variance components were statistically significant. We also see the correlation of genetic and environmental influences across outcomes. If the genetic/environmental correlation between two traits is 1 , all genetic/environmental variance in trait 1 and 2 has a common base. If the correlation is 0 , the genetically/environmentally based variance between trait 1 and 2 are independent. Shared environmental effects on education and age at first birth correlated to 1 meaning that there are no shared environmental effects for age at first birth independent of shared environmental influences on education. Also a model that constrained shared environmental effects for education and age at first birth to be the same had an equal statistical fit as the model freely estimating the parameter (not listed). Importantly, genetic effects across both outcomes do not significantly correlate (0.14), implying that family background factors causing a spurious association between education and age at first birth were mainly environmental in 
nature. Note that the direct effects from the $M Z$ twin-fixed effects estimates reduces to 0.07 in this model specification. In the ACE-beta model, the direct link between education and age at first birth explained 16 percent of the association, shared environmental influences amongst siblings 62 percent, and shared genetic influences 22 percent (see Si 5-5 and SI 5-6 for details).

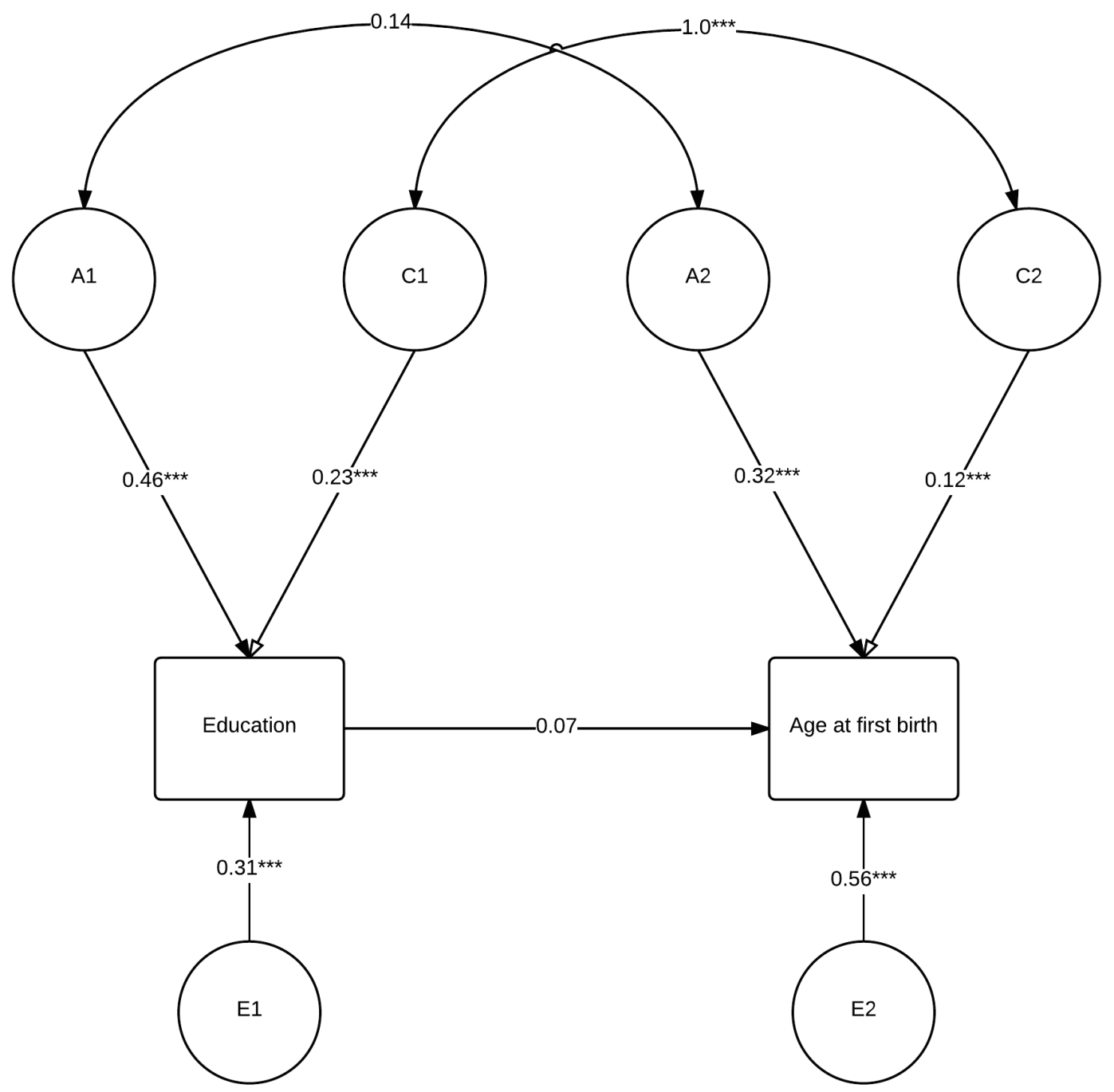

Figure 5-3 Correlated factor model depicting standardized estimate of genetic (A) and shared environmental $(C)$ influences on education and age at first birth, the correlation of these influences across traits as well as a causal effect of education on the age at first birth

Notes: * $\mathrm{p}<0.05,{ }^{* *} \mathrm{p}<0.01,{ }^{* * *} \mathrm{p}<0.001$, two-sided

Source: TwinsUK, own calculations. 


\subsection{Discussion}

The supposed causal effect of education on fertility postponement has become a parsimonious and powerful explanation for demographers, not only for the fact that higher educated women have their first baby at later ages, but also for the general postponement of childbearing during the second half of the $20^{\text {th }}$ century due to the educational expansion (Ní Bhrolcháin \& Beaujouan, 2012). In this study, we challenge the claim that education causally influences age at first birth and consequently that the educational expansion is the main cause of recent fertility postponement. We present within-twin (fixed effects) and novel biometric models using a unique dataset of female twins from the UK. We estimate the causal effect of education on age at first birth, and the extent to which environmental and genetic factors cause a spurious association. We find a reduced effect of education on age at first birth in the within-twin design compared to standard regression models. Complementary biometric analyses reveal that the association between education and age at first birth is to a large extent caused by social family background effects, whereas genetic inheritance plays only a small role. These results suggest that the prevailing view of a strong causal effect of education has no merit and needs to be revised.

In our study, the effect of all family factors shared among siblings explained up to two thirds of the observed association between education and age at first birth, and only a small part of the effect is due to genetic dispositions. This finding provides a strong justification for continued research into the role of family background effects on education and fertility. However, the twin models we present do not give insights into which family background factors are important for both education and age at first birth. Previous investigations have pointed to socioeconomic status of the parents (van Doorn et al., 2011) and parental demographic behavior (e. g. Lappegård \& Rønsen, 2005; Marini, 1985; Nisén \& Myrskylä, 2014; Rijken \& Liefbroer, 2009). Recent investigations, also show that social ties such as friends (Balbo \& Barban, 2014) influence fertility timing and that siblings influence each other (Lyngstad \& Prskawetz, 2010). Given that measured family characteristics typically only account for a small part of the explained variance (e. g. Nisén \& Myrskylä, 2014), and parents are important in choosing who to befriend and date, 
one direction to better understand the role of the family in fertility behavior is in further study of social and network dynamics (of the family).

Since the central goals of demographic research are the discovery, description and explanation of phenomena at the population-level, individual-level approaches have been criticised for their inattention to the core perspective in demography (Bhrolcháin \& Dyson, 2007; R. Lee, 2001; Smith, 2003). Ní Bhrolcháin and Dyson (2007) proposed a catalogue of ten criteria for making causal claims when linking macro phenomena such as the educational expansion to the postponement of fertility. These include: the temporal order of events, the no-cause-no-effect-rule, the proposition of mechanisms, and the proportionality of the scale of cause and effects. In a comparable study with nearly identical findings at the population level to those of our study, Ní Bhrolcháin and Beaujouan (2012) assign an important role to education for fertility postponement, which underscores the importance of education for fertility projections (Lutz et al., 2014).

A central goal of our study was to link our findings at the individual level to the population level (Billari, 2015). The main ways in which our approach is distinct are that the unit of analysis changes from populations to individuals and that we present and 'exception' of the no-cause-no-effect rule, since we postulate that fertility postponement is not a mono- but a multi-causal phenomenon. Our findings mainly challenge those from Ní Bhrolcháin and Beaujouan (2012) with respect to the criterion of proportionality, as we find that increasing educational attainment can only explain a fraction of the trend in age at first birth-if we integrate results from the individual level into our projection. Perhaps the educational expansion serves as a proxy in many studies for other simultaneous historical developments that are not directly measured. An extensive review by Hobcraft (1996) identified changes in sexual norms and family planning, the dramatic economic crises during the 1970s and 80s, and the introduction of the 'pill' as reasons for declining fertility levels and fertility postponement within the UK. In that sense, we agree that, for the relationship under study, the question is not 'what causes fertility postponement?', but 'to what extent does the educational expansion (and other factors) cause fertility postponement?'. Overall, the role of education as a useful predictor of future fertility trends is in doubt. 
Previous investigations on both the causal effect of education on age at first birth and the role of genetic versus environmental family background factors produced mixed findings, and our study may contribute to explaining these inconsistencies. First, the nuanced interpretation of the role of education fits with two previous studies. Neiss et al. (2002) and Rodgers et al. (2008) show for the US and Denmark that the association between education and age at first birth is completely absorbed by latent family influences. Nisén and Myrskylä (2013) and Amin and Behrman (2014), however, find a significant link between both outcomes net of family influences. Our study suggests that a causal effect of education on age at first birth exists, however, that it is small, and therefore might have remained undetected in former investigations. Note that in the current study, the causal effect estimated from within-identical twins - and therefore also in the ACE-beta model is not statistically distinguishable from zero, and only if we pool identical and fraternal twins - which have nearly identical estimates - do we detect the causal link.

It also needs to be mentioned that findings on the role of the genetic component in age at first birth in general are mixed in the literature. We find that around one third of the variance in age at first birth is explained by additive genetic effects. This is similar to e.g. studies from the US (Byars et al., 2010), Australia (Kirk et al., 2001) or Finland (Nisén et al., 2013). However, other investigations from the US (Neiss et al., 2002) and Denmark (Rodgers et al., 2008) find no significant genetic influences. Differences in genetic effects on fertility across countries and within countries over time exist - also within the UK (Tropf, Barban, et al., 2015) - and may be due to gene-environment interaction (Kohler et al., 2006; Mills \& Tropf, 2016). Further investigation into the genetic pathways to fertility is needed, also in order to better understand patterns of gene-environment interaction across populations also including molecular genetic information (Tropf, Stulp, et al., 2015).

The within (identical) twin approach we applied forms a useful tool to establish a causal relationship between two variables yet it has its limitations. The most critical assumption is presumably that variation in education within twin pairs is uncorrelated with variation in age at first birth. The question arises: what makes (identical) twins different in educational attainment? If for example health issues lead to early school dropout and delayed fertility, the causal effect estimated from 
felix-tropf.com

the within twin models will be smaller than the true effect. If the presence of a partner influences career and fertility aspirations towards longer educational and later fertility, the true causal effect would be smaller than the estimate from the within-twin models. Additionally, measurement error can downwardly bias the estimates (see for discussion Amin et al., 2013). However, conservative predictions based on estimates from the OLS models suggest that the educational expansion cannot account for a large part of observed fertility postponement. Nevertheless, we advise caution for the interpretation of our findings insofar as we find mean differences in age at first birth across TwinsUK and the GHS data. Alternative designs can validate our findings in future research. Desirable approaches include quasi-experimental designs using instrument variables (for a recent discussion see Amin et al., 2015; Boardman \& Fletcher, 2015).

In general, our study challenges the common approach of explaining differences and trends in fertility timing mainly by invoking educational differences. Differences between families and societal changes and upheavals across time can have a strong impact on fertility timing, which may be mistakenly attributed to the causal influence of just one factor. This study shows that fertility timing is the result of a complex interplay of environmental and genetic influences. We conclude that (the rise in) educational attainment alone cannot explain differences in fertility timing (between cohorts). 


\section{Literature}

Ajzen, I. (1991). The theory of planned behavior. Organizational Behavior and Human Decision Processes, 50(2), 179-211.

Allison, P. D., \& Christakis, N. (2006). Fixed-effects methods for the analysis of nonrepeated events. Sociological Methodology, 36(1), 155172. http:/ / doi.org/10.1111/j.1467-9531.2006.00177.x

Amin, V., \& Behrman, J. R. (2014). Do more-schooled women have fewer children and delay childbearing? Evidence from a sample of US twins. Journal of Population Economics, 27(1), 1-31. http: / / doi.org/10.1007/ s00148-013-0470-z

Amin, V., Behrman, J. R., Kohler, H.-P., Xiong, Y., \& Zhang, J. (2015). Causal inferences: Identical twins help and clarity about necessary assumptions is critical. Social Science $\mathcal{E}$ Medicine, 127, 201-202. http: / / doi.org/10.1016/j.socscimed.2014.12.012

Amin, V., Behrman, J. R., \& Spector, T. D. (2013). Does more schooling improve health outcomes and health related behaviors? Evidence from UK twins. Economics of Education Review, 35, 134-148.

Amuedo-Dorantes, C., \& Kimmel, J. (2005). The motherhood wage gap for women in the United States: The importance of college and fertility delay. Review of Economics of the Household, 3(1), 17-48.

Anderson, C. A., Duffy, D. L., Martin, N. G., \& Visscher, P. M. (2007). Estimation of variance components for age at menarche in twin families. Behavior Genetics, 37(5), 668-677.

Andersson, G. (2000). The Impact of Labour-Force Participation on Childbearing Behaviour: Pro-Cyclical Fertility in Sweden during the 1980s and the 1990s. European Journal of Population, 16(4), 293-333. http: / / doi.org/10.1023/ A:1006454909642

Anderton, D. L., Tsuya, N. O., Bean, L. L., \& Mineau, G. P. (1987). Intergenerational Transmission of Relative Fertility and Life Course Patterns. Demography, http: / / doi.org/10.2307/ 2061386 24(4), $467-480$.

Andrew, T., Hart, D. J., Snieder, H., de Lange, M., Spector, T. D., \& MacGregor, A. J. (2001). Are twins and singletons comparable? A study of disease-related and lifestyle characteristics in adult women. Twin Research: The Official Journal of the International Society for Twin 
Studies, 4(6), 464-77. Retrieved from http: / / www.ncbi.nlm.nih.gov/ pubmed /11780939

Balbo, N., \& Barban, N. (2014). Does fertility behavior spread among friends? American Sociological Review, 79(3), 412-431.

Balbo, N., Billari, F. C., \& Mills, M. C. (2013). Fertility in advanced societies: A review of research. European Journal of Population/Revue Européenne de Démographie, 29(1), 1-38.

Balbo, N., \& Mills, M. C. (2011). Social capital and pressure in fertility decision-making: second and third births in France, Germany and Bulgaria. Population Studies, 65(3), 335-351.

Barber, J. S. (2000). Intergenerational influences on the entry into parenthood: Mothers' preferences for family and nonfamily behavior. Social Forces, 79(1), 319-348.

Beauchamp, J. P. (2016). Genetic evidence for natural selection in humans in the contemporary United States. Proc Natl Acad Sci.

Benjamin, D. J., Cesarini, D., van der Loos, M. J. H. M., Dawes, C. T., Koellinger, P. D., Magnusson, P. K. E., ... Johannesson, M. (2012). The genetic architecture of economic and political preferences. Proceedings of the National Academy of Sciences, 109(21), 8026-8031.

Bhrolcháin, M. N., \& Dyson, T. (2007). On Causation in Demography: Issues and Illustrations. Population and Development Review, 33(1), 136. http: / / doi.org/10.1111/j.1728-4457.2007.00157.x

Billari, F. (2015). Integrating macro-and micro-level approaches in the explanation of population change. Population Studies, 69(Suppl 1), 11-20.

Boardman, J. D., \& Fletcher, J. M. (2015). To cause or not to cause? That is the question, but identical twins might not have all of the answers. Social Science $\mathcal{E}$ Medicine, 127, 198-200. http: / / doi.org/10.1016/j.socscimed.2014.10.013

Boker, S., Neale, M., Maes, H., Wilde, M., Spiegel, M., Brick, T., ... Bates, T. (2011). OpenMx: an open source extended structural equation modeling framework. Psychometrika, 76(2), 306-317.

Bolund, E., Hayward, A., Pettay, J., \& Lummaa, V. (2015). Effects of the demographic transition on the genetic variances and covariances of human life-history traits. Evolution, 69(3), 747-755.

Bongaarts, J. (1978). A Framework for Analyzing the Proximate Determinants of Fertility. Population and Development Review, 4(1), 
105-132. http: / / doi.org/10.2307/1972149

Bongaarts, J. (1982). The Fertility-Inhibiting Effects of the Intermediate Fertility Variables. Studies in Family Planning, 13(6-7), 179-189.

Bongaarts, J. (1983). The proximate determinants of natural marital fertility. Determinants of Fertility in Developing Countries: A Summary of Knowledge, (15), 85.

Bongaarts, J., \& Feeney, G. (2000). On the quantum and tempo of fertility: Reply. Population and Development Review, 26(3), 560-564.

Boomsma, D., Busjahn, A., \& Peltonen, L. (2002). Classical twin studies and beyond. Nature Reviews Genetics, 3(11), 872-882.

Booth, A. L., \& Kee, H. J. (2009). Intergenerational Transmission of Fertility Patterns*. Oxford Bulletin of Economics and Statistics, 71(2), 183-208.

Branigan, A. R., McCallum, K. J., \& Freese, J. (2013). Variation in the heritability of educational attainment: An international metaanalysis. Social Forces, 92(1), 109-140.

Bras, H., Bavel, J. Van, \& Mandemakers, K. (2013). Unraveling the intergenerational transmission of fertility: genetic and sharedenvironment effects during the demographic transition in the Netherlands, 1810-1910. The History of the Family, 18(2), 116-134.

Bray, H. (2008). 2006-based national population projections for the U.K. and constituent countries. Population Trends, (131), 8-18.

Briley, D., Harden, K., \& Tucker-Drob, E. (2015). Genotypex Cohort Interaction on Completed Fertility and Age at First Birth. Behavior Genetics, 45(1), 71-83.

Bulik-Sullivan, B., Finucane, H. K., Anttila, V., Gusev, A., Day, F. R., Loh, P.-R., ... Neale, B. M. (2015). An atlas of genetic correlations across human diseases and traits. Nature Genetics, 47(11), 1236-1241. http: / / doi.org/10.1038/ng.3406

Byars, S. G., Ewbank, D., Govindaraju, D. R., \& Stearns, S. C. (2010). Natural selection in a contemporary human population. Proceedings of the National Academy of Sciences, 107(suppl 1), 1787-1792.

Carey, J., \& Vaupel, J. (2005). Biodemography. In Handbook of Population (pp. 625-658). New York: Kluwer Academic Publishers-Plenum Publishers.

Chabris, C. F., Lee, J. J., Cesarini, D., Benjamin, D. J., \& Laibson, D. I. (2015). The Fourth Law of Behavior Genetics. Current Directions in 
Psychological Science, 24(4), $304-312$. http: / / doi.org/10.1177/ 0963721415580430

Christensen, K., Kohler, H.-P., \& Basso, O. (2003). The correlation of fecundability among twins: evidence of a genetic effect on fertility? Epidemiology, 14(1), 60-64.

Cleveland, W. S. (1979). Robust locally weighted regression and smoothing scatterplots. Journal of the American Statistical Association, 74(368), 829-836.

Conley, D. (2009). The promise and challenges of incorporating genetic data into longitudinal social science surveys and research. Biodemography and Social Biology, 55(2), 238-251.

Conley, D., \& Domingue, B. (2016). The Bell Curve Revisited: Testing Controversial Hypotheses with Molecular Genetic Data. http: / / doi.org/10.15195/v3.a23

Conley, D., Laidley, T., Belsky, D. W., Fletcher, J. M., Boardman, J. D., \& Domingue, B. W. (2016). Assortative mating and differential fertility by phenotype and genotype across the 20th century. Proceedings of the National Academy of Sciences, 1523592113. http: / / doi.org/10.1073/ pnas.1523592113

Conley, D., Rauscher, E., Dawes, C., Magnusson, P. K. E., \& Siegal, M. L. (2013). Heritability and the equal environments assumption: evidence from multiple samples of misclassified twins. Behavior Genetics, 43(5), 415-426.

Consortium, I. H. 3. (2010). Integrating common and rare genetic variation in diverse human populations. Nature, 467(7311), 52-58.

Courtiol, A., Rickard, I., \& Lummaa, V. (2013). The demographic transition influences variance in fitness and selection on height and BMI in rural Gambia. Current Biology, 23(10), 884-889.

Courtiol, A., Tropf, F. C., \& Mills, M. C. (2016). When genes and environment disagree: Making sense of trends in recent human evolution. Proceedings of the National Academy of Sciences, 113(28), 7693-7695. http:/ / doi.org/10.1073/pnas.1608532113

Cui, J. S., Hopper, J. L., \& Harrap, S. B. (2003). Antihypertensive treatments obscure familial contributions to blood pressure variation. Hypertension, 41(2), 207-210. http: / / doi.org/10.1161/01.HYP.0000044938.94050.E3

D'Onofrio, B. M., \& Lahey, B. B. (2010). Biosocial influences on the family: A decade review. Journal of Marriage and Family, 72(3), 762- 
782.

D'Onofrio, B. M., Lahey, B. B., Turkheimer, E., \& Lichtenstein, P. (2013). Critical Need for Family-Based, Quasi-Experimental Designs in Integrating Genetic and Social Science Research. American Journal of Public Health, 103(S1),

S46-S55. http: / / doi.org/10.2105/ AJPH.2013.301252

Davis, K., \& Blake, J. (1956). Social Structure and Fertility: An Analytic Framework. Economic Development and Cultural Change, 4(3), 211-235. http: / / doi.org/10.1086/ 449714

Daw, J., \& Guo, G. (2011). The influence of three genes on whether adolescents use contraception, USA 1994-2002. Population Studies, 65(3), 253-271.

Day, F., Ruth, K., \& Thompson, D. (2015). Large-scale genomic analyses link reproductive aging to hypothalamic signaling, breast cancer susceptibility and BRCA1-mediated DNA repair. Nature Genetics, 47(11), 1294-1303.

Devlin, B., Daniels, M., \& Roeder, K. (1997). The heritability of IQ. Nature, 388(6641), 468-471.

Dunne, M. P., Martin, N. G., Statham, D. J., Slutske, W. S., Dinwiddie, S. H., Bucholz, K. K., ... Heath, A. C. (1997). Genetic and environmental contributions to variance in age at first sexual intercourse. Psychological Science, 8(3), 211.

Elks, C., Perry, J., Sulem, P., Chasman, D., Franscechini, N., \& Al, E. (2010). Thirty new loci for age at menarche identified by a metaanalysis of genome-wide association studies. Nature Genetics, 42(12), 1077-1085.

Ellegren, H., \& Parsch, J. (2007). The evolution of sex-biased genes and sex-biased gene expression. Nature Reviews Genetics, 8(9), 689-698.

Felson, J. (2014). What can we learn from twin studies? A comprehensive evaluation of the equal environments assumption. Social Science Research, 43(December), 184-199. http:/ / doi.org/10.1016/j.ssresearch.2013.10.004

Fisher, R. A. (1930). The Genetical Theory of Natural Selection. Oxford University Press.

Foster, C. (2000). The limits to low fertility: a biosocial approach. Population and Development Review, 26(2), 209-234. http: / / doi.org/10.1111/j.1728-4457.2000.00209.x 
Freese, J. (2008). Genetics and the Social Science Explanation of Individual Outcomes1. American Journal of Sociology, 114(S1), S1-S35.

Furstenberg Jr., F. F., Levine, J. A., \& Brooks-Gunn, J. (1990). The Children of Teenage Mothers: Patterns of Early Childbearing in Two Generations. Family Planning Perspectives, 22(2), 54-61. http: / / doi.org/10.2307 / 2135509

Goisis, A. (2013). Childbearing postponement and child wellbeing in the U.K: reconciling and integrating different perspectives. The London School of Economics and Political Science (LSE).

Goldstein, J. R., Sobotka, T., \& Jasilioniene, A. (2009). The End of "Lowest-Low" Fertility? Population and Development Review, 35(4), 663-699.

Goodman, A., Koupil, I., \& Lawson, D. W. (2012). Low fertility increases descendant socioeconomic position but reduces long-term fitness in a modern post-industrial society. Proceedings of the Royal Society B: Biological Sciences, 279(1746), 4342-4351. http:/ / doi.org/10.1098/ rspb.2012.1415

Guo, G. (2005). twin studies: what can they tell us about nature and nurture? Contexts, 4(3), 43.

Guo, G., Tong, Y., \& Cai, T. (2008). Gene by Social-Context Interactions for Number of Sexual Partners Among White Male Youths: Genetics-Informed Sociology. AJS; American Journal of Sociology, 114(Suppl), S36.

Gustafsson, S., Kenjoh, E., \& Wetzels, C. (2002). The role of education on postponement of maternity in Britain, Germany, the Netherlands and Sweden. In E. Ruspini \& A. Dale (Eds.), The Gender Dimension of Social Change. The Contribution of Dynamic Research to the Study of Women's Life Courses (pp. 55-79). Bristol: Policy Press.

Happel, S. K., Hill, J. K., \& Low, S. A. (1984). An economic analysis of the timing of childbirth. Population Studies, 38(2), 299-311.

He, C., Kraft, P., Chen, C., Buring, J. E., Paré, G., Hankinson, S. E., ... Chasman, D. I. (2009). Genome-wide association studies identify loci associated with age at menarche and age at natural menopause. Nature Genetics, 41(6), 724-728.

Hettema, J. M., Neale, M. C., \& Kendler, K. S. (1995). Physical similarity and the equal-environment assumption in twin studies of psychiatric disorders. Behavior Genetics, 25(4), 327-335. 
Hewitt, J. (2012). Editorial policy on candidate gene association and candidate gene-by-environment interaction studies of complex traits. Behavior Genetics, 41(1), 1-2.

Hindorff, L. A., Sethupathy, P., Junkins, H. A., Ramos, E. M., Mehta, J. P., Collins, F. S., \& Manolio, T. A. (2009). Potential etiologic and functional implications of genome-wide association loci for human diseases and traits. Proceedings of the National Academy of Sciences, 106(23), 9362-9367.

Hobcraft, J. (1996). Fertility in England and Wales: a fifty-year perspective. Population Studies, 50(3), 485-524.

Hobcraft, J. (2006). The ABC of demographic behaviour: How the interplays of alleles, brains, and contexts over the life course should shape research aimed at understanding population processes. Population Studies, 60(2), 153-187.

Hobcraft, J., \& Little, R. J. a. (1984). Fertility exposure analysis: A new method for assessing the contribution of proximate determinants to fertility differentials. Population Studies, 38(1), 21-45. http: / / doi.org/10.1080/00324728.1984.10412821

Hoekstra, C., Zhao, Z. Z., Lambalk, C. B., Willemsen, G., Martin, N. G., Boomsma, D. I., \& Montgomery, G. W. (2008). Dizygotic twinning. Human Reproduction Update, 14(1), 37-47.

Hoem, B. (2000). Entry into Motherhood in Sweden: The Influence of Economic Factors on the Rise and Fall in Fertility, 1986-1997. Demographic Research, 2. http: / / doi.org/10.4054/ DemRes.2000.2.4

Holst, K. K., \& Budtz-Jørgensen, E. (2012). Linear latent variable models: the lava-package. Computational Statistics, 1-68.

Horwitz, A. V, Videon, T. M., Schmitz, M. F., \& Davis, D. (2003). Rethinking twins and environments: Possible social sources for assumed genetic influences in twin research. Journal of Health and Social Behavior, 44(2), 111-129.

Hughes, K., \& Burleson, M. H. (2000). Evolutionary Causes of Genetic Variation in Fertility and other Fitness Traits. In J. L. Rodgers, D. C. Row, \& W. B. Miller (Eds.), (1st ed., pp. 7-33). Massachusetts: Kluwer Academic Publisher.

Investigators, A. (1989). The atherosclerosis risk in community (aric) study: design and objectives. American Journal of Epidemiology, 129(4), 687-702.

Ioannidis, J. P., Ntzani, E. E., Trikalinos, T. A., \& Contopoulos-Ioannidis, 
D. G. (2001). Replication validity of genetic association studies. Nature Genetics, 29(3), 306-9. http: / / doi.org/10.1038/ng749

Jackson, R., Chambless, L., Yang, K., K., Byrne, T., Watson, R., \& Folsom, A. (1996). Differences between respondents and nonrespondents in a multicenter community-based study vary by gender and ethnicity. Journal of Clinical Epidemiology, 49(12), 1441-1446.

Jæger, M. M. (2012). The Extended Family and Children's Educational Success. American Sociological Review, 77(6), 903-922.

Jenkins, A., \& Sabates, R. (2007). The classification of qualifications in social surveys (No. 2). London. Retrieved from http:/ / eprints.ioe.ac.uk/5699/1/Jenkins2007Classification_2007_2.p df

Joffe, M. (2010). What has happened to human fertility? Human Reproduction (Oxford, England), 25(2), 295-307.

Joshi, H. (2002). Production, reproduction, and education: Women, children, and work in a British perspective. Population and Development Review, 28(3), 445-474.

Juster, F., \& Suzman, R. (1995). An overview of the Health and Retirement Study. Journal of Human Resources, S7-S56.

Kahn, J. R., \& Anderson, K. E. (1992). Intergenerational patterns of teenage fertility. Demography, 29(1), 39-57. http: / / doi.org/10.2307/ 2061362

Katus, K., Puur, A., \& Põldma, A. (2004). Population-related Policies in Estonia in the 20th Century: Stages and Turning Points. Finnish Yearbook of Population Research, 40, 73-103.

Kiernan, K. (2004). Unmarried cohabitation and parenthood in Britain and Europe. Law \& Policy, 26(1), 33-55.

Kirk, K. M., Blomberg, S. P., Duffy, D. L., Heath, A. C., Owens, I. P. F., \& Martin, N. G. (2001). Natural selection and quantitative genetics of life-history traits in Western women: a twin study. Evolution, 55(2), 423-435.

Klijs, B., Scholtens, S., \& Mandemakers, J. (2015). Representativeness of the LifeLines Cohort Study. PloS One, 10(9), e0137203.

Kneale, D., \& Joshi, H. (2008). Postponement and childlessness: Evidence from two British cohorts. Demographic Research, 19, 1935-1964.

Kohler, H.-P., Behrman, J. R., \& Schnittker, J. (2011). Social science 
methods for twins data: Integrating causality, endowments, and heritability. Biodemography and Social Biology, 57(1), 88-141.

Kohler, H.-P., Billari, F. C., \& Ortega, J. A. (2002). The emergence of lowest-low fertility in Europe during the 1990s. Population and Development Review, 28(4), 641-680.

Kohler, H.-P., \& Rodgers, J. L. (1999). DF-like analyses of binary, ordered, and censored variables using probit and tobit approaches. Behavior Genetics, 29(4), 221-232.

Kohler, H.-P., \& Rodgers, J. L. (2003). Education, fertility and heritability: Explaining a paradox. In R. A. Bulatao \& K. W. Wachter (Eds.), Offspring: Human fertility behavior in biodemographic perspective (pp. 46-90). National Academy Press.

Kohler, H.-P., Rodgers, J. L., \& Christensen, K. (1999). Is fertility behavior in our genes? Findings from a Danish twin study. Population and Development Review, 25(2), 253-288.

Kohler, H.-P., Rodgers, J. L., \& Christensen, K. (2002). Between nurture and nature: the shifting determinants of female fertility in Danish twin cohorts. Biodemography and Social Biology, 49(3-4), 218-248.

Kohler, H.-P., Rodgers, J. L., Miller, W. B., Skytthe, A., \& Christensen, K. (2006). Bio-social determinants of fertility. International Journal of Andrology, 29(1), 46-53.

Komlos, J., \& Lauderdale, B. E. (2007). The mysterious trend in American heights in the 20th century. Annals of Human Biology, 34(2), 206-215.

Kosova, G., Abney, M., \& Ober, C. (2010). Heritability of reproductive fitness traits in a human population. Proceedings of the National Academy of Sciences, 107(suppl 1), 1772-1778.

Lappegård, T., \& Rønsen, M. (2005). The multifaceted impact of education on entry into motherhood. European Journal of Population/Revue Européenne de Démographie, 21(1), 31-49.

Lee, R. (2001). Demography Abandons Its Core Demography is Changing: Less Macro, More Micro. Retrieved July 27, 2016, from http://www.ceda.berkeley.edu/papers/rlee/

Lee, S. H., Wray, N. R., Goddard, M. E., \& Visscher, P. M. (2011). Estimating missing heritability for disease from genome-wide association studies. The American Journal of Human Genetics, 88(3), 294-305.

Lee, S. H., Yang, J., Goddard, M. E., Visscher, P. M., \& Wray, N. R. 
(2012). Estimation of pleiotropy between complex diseases using single-nucleotide polymorphism-derived genomic relationships and restricted maximum likelihood. Bioinformatics, 28(19), 2540-2542.

Leitsalu, L., Haller, T., Esko, T., Tammesoo, M. L., Alavere, H., Snieder, H., ... Metspalu, A. (2015). Cohort profile: Estonian biobank of the Estonian genome center, university of Tartu. International Journal of Epidemiology, 44(4), 1137-1147. http:/ / doi.org/10.1093/ije/ dyt268

Leridon, H. (2008). A new estimate of permanent sterility by age: sterility defined as the inability to conceive. Population Studies, 62(1), 15-24.

Lesthaeghe, R. (1995). The Second Demographic Transition in Western Countries: An Interpretation. In Gender and Family Change in Industrialized Countries (pp. 17-62). http: / / doi.org/10.2307/1534906

Levine, P., \& Bashford, A. (2010). Introduction: Eugenics and the modern world. The Oxford Handbook of the History of Eugenics.

Lichtenstein, P., Sullivan, P. F., Cnattingius, S., Gatz, M., Johansson, S., Carlström, E., ... Klareskog, L. (2006). The Swedish Twin Registry in the third millennium: an update. Twin Research and Human Genetics, 9(06), 875-882.

Liefbroer, A., \& Corijn, M. (1999). Who, what, where, and when? Specifying the impact of educational attainment and labour force participation on family formation. Journal of Population/Revue Européenne de Démographie, 15(1), 45-75.

Liu, Y.-Z., Guo, Y.-F., Wang, L., Tan, L.-J., Liu, X.-G., Pei, Y.-F., ... Yu, N. (2009). Genome-wide association analyses identify SPOCK as a key novel gene underlying age at menarche. PLoS Genetics, 5(3), e1000420.

Loehlin, J. C. (1996). The Cholesky approach: A cautionary note. Behavior Genetics, 26(1), 65-69. http: / / doi.org/10.1007/BF02361160

Lutz, W., Butz, W., \& Samir, K. (2014). World population and human capital in the twenty-first century.

Lynch, M., \& Walsh, B. (1998). Genetics and analysis of quantitative traits. Sinauer: Sunderland.

Lyngstad, T. H., \& Prskawetz, A. (2010). Do Siblings' Fertility Decisions Influence each Other? Demography, 47(4), 923-934.

Manolio, T. A., Collins, F. S., Cox, N. J., Goldstein, D. B., Hindorff, L. A., Hunter, D. J., ... Chakravarti, A. (2009). Finding the missing 
heritability of complex diseases. Nature, 461(7265), 747-753.

Marini, M. M. (1985). Determinants of the timing of adult role entry. Social Science Research, 14(4), 309-350.

Martin, S. P. (2000). Diverging fertility among U.S. women who delay childbearing past age 30. Demography, 37(4), 523-533.

Mbarek, H., Steinberg, S., Nyholt, D. R., Gordon, S. D., Miller, M. B., McRae, A. F., ... Boomsma, D. I. (2016). Identification of Common Genetic Variants Influencing Spontaneous Dizygotic Twinning and Female Fertility. American Journal of Human Genetics. http: / / doi.org/10.1016/j.ajhg.2016.03.008

McCrary, J., \& Royer, H. (2011). The effect of female education on fertility and infant health: Evidence from school entry policies using exact date of birth. American Economic Review, 101(1), 158-195.

Mealey, L., \& Segal, N. L. (1993). Heritable and environmental variables affect reproduction-related behaviors, but not ultimate reproductive success. Personality and Individual Differences, 14(6), 783-794.

Mehta, D., Tropf, F. C., Gratten, J., Bakshi, A., Zhu, Z., Bacanu, S.-A., ... $\mathrm{Wu}$, J. Q. (2016). Evidence for Genetic Overlap Between Schizophrenia and Age at First Birth in Women. JAMA Psychiatry, 73(5), 497-505. http: / / doi.org/10.1001/jamapsychiatry.2016.0129

Miller, W. B. (1992). Personality traits and developmental experiences as antecedents of childbearing motivation. Demography, 29(2), 265-285.

Miller, W. B. (1994). Childbearing motivations, desires, and intentions: A theoretical framework. Genetic, Social $\mathcal{E}$ General Psychology Monographs, 120(2), 223-258.

Miller, W. B., Bard, D. E., Pasta, D. J., \& Rodgers, J. L. (2010). Biodemographic modeling of the links between fertility motivation and fertility outcomes in the NLSY79. Demography, 47(2), 393-414.

Miller, W. B., \& Pasta, D. J. (1994). The Psychology of Child Timing: A Measurement Instrument and a Model1. Journal of Applied Social Psychology, 24(3), 218-250.

Mills, M. C. (2011). Introducing survival and event history analysis. Sage Publications.

Mills, M. C., \& Blossfeld, H.-P. (2005). Globalization, Uncertainty and the Early Life Course: A Theoretical Framework: 1-24. In Globalization, Uncertainty and Youth in Society. London: Routledge.

Mills, M. C., Blossfeld, H.-P., \& Klijzing, E. (2005). Becoming an adult in 
uncertain times. In Globalization, Uncertainty and Youth in Society. London: Routledge.

Mills, M. C., Rindfuss, R. R., McDonald, P., \& te Velde, E. (2011). Why do people postpone parenthood? Reasons and social policy incentives. Human Reproduction Update, 17(6), 848-860. http: / / doi.org/10.1093/humupd / dmr026

Mills, M. C., \& Tropf, F. C. (2016). The Biodemography of Fertility: A Review and Future Research Frontiers. Kölner Zeitschrift Für Soziologie Und Sozialpsychologie, 55(Special Issues Demography), 397424.

Milot, E., Mayer, F. M., Nussey, D. H., Boisvert, M., Pelletier, F., \& Réale, D. (2011). Evidence for evolution in response to natural selection in a contemporary human population. Proceedings of the National Academy of Sciences, 108(41), 17040-17045.

Milot, E., \& Pelletier, F. (2013). Human Evolution: New Playgrounds for Natural Selection. Current Biology, 23(10), R446-R448.

Moayyeri, A., Hammond, C., \& Spector, T. D. (2013). Cohort profile: TwinsUK and healthy ageing twin study. International Journal of Epidemiology, 42(1), 76-85.

Montgomery, G. W., Zondervan, K. T., \& Nyholt, D. R. (2014). The future for genetic studies in reproduction. Molecular Human Reproduction, 20(1), 1-14.

Morrissey, M. B., Kruuk, L. E. B., \& Wilson, A. J. (2010). The danger of applying the breeder's equation in observational studies of natural populations. Journal of Evolutionary Biology, 23(11), 2277-2288.

Mousseau, T. A., \& Roff, D. A. (1987). Natural selection and the heritability of fitness components. Heredity, 59(Pt 2), 181-197.

Murphy, M. (1993). The contraceptive pill and women's employment as factors in fertility change in Britain 1963-1980: a challenge to the conventional view. Population Studies, 47(2), 221-243.

Murphy, M. (1999). Is the relationship between fertility of parents and children really weak? Biodemography and Social Biology, 46(1-2), 122145 .

Murphy, M., \& Wang, D. (2001). Family-level continuities in childbearing in low-fertility societies. European Journal of Population, 17(1), 75-96. http:/ / doi.org/10.1023 / A:1010744314362

Neale, M. C., \& Cardon, L. R. (1992). Methodology for genetic studies of 
twins and families. Dordrecht, the Netherlands: Kluwer Academic Publishers.

Neiss, M., Rowe, D. C., \& Rodgers, J. L. (2002). Does education mediate the relationship between IQ and age of first birth? A behavioural genetic analysis. Journal of Biosocial Science, 34(2), 259-276.

Ní Bhrolcháin, M., \& Beaujouan, É. (2012). Fertility postponement is largely due to rising educational enrolment. Population Studies, 66(3), 311-327.

Nisén, J., Martikainen, P., Kaprio, J., \& Silventoinen, K. (2013). Educational Differences in Completed Fertility: A Behavioral Genetic Study of Finnish Male and Female Twins. Demography, 50(4), $1-22$.

Nisén, J., \& Myrskylä, M. (2014). Effect of family background on the educational gradient in lifetime fertility of Finnish women born 1940-50. Population Studies, 68(3), 321-337.

Office National Statistics. (2013). Cohort Fertility, Table 2. Retrieved from http: / / www.ons.gov.uk/ons/publications/re-referencetables.html?edition=tcm:77-263133

Ooki, S., \& Asaka, A. (2004). Zygosity diagnosis in young twins by questionnaire for twins' mothers and twins' self-reports. Twin Research, 7(1), 5-12.

Oppenheimer, V. K. (1994). Women's Rising Employment and the Future of the Family in Industrial Societies. Population and Development Review, 20(2), 293-342. http:/ / doi.org/10.2307/2137521

Oreopoulos, P. (2006). Estimating average and local average treatment effects of education when compulsory schooling laws really matter. The American Economic Review, 96(1), 152-175.

Painter, J. N., Anderson, C. A., Nyholt, D. R., Macgregor, S., Lin, J., Lee, S. H., ... Guo, Q. (2011). Genome-wide association study identifies a locus at 7p15. 2 associated with endometriosis. Nature Genetics, 43(1), $51-54$.

Parsch, J., \& Ellegren, H. (2013). The evolutionary causes and consequences of sex-biased gene expression. Nature Reviews Genetics, 14(2), 83-87.

Pasta, D., \& Miller, W. B. (2000). A heritability study of childbearing motivation. In Genetic Influences on Human Fertility and Sexuality (pp. 107-120). Springer US. 
Pearson, K., Lee, A., \& Bramley-Moore, L. (1899). Mathematical contributions to the theory of evolution. VI. Genetic (reproductive) selection: Inheritance of fertility in man, and of fecundity in thoroughbred racehorses. Philosophical Transactions of the Royal Society of London.Series A, Containing Papers of a Mathematical or Physical Character, 192, 257-330.

Perry, J. R. B., Stolk, L., Franceschini, N., Lunetta, K. L., Zhai, G., McArdle, P. F., ... Boerwinkle, E. (2009). Meta-analysis of genomewide association data identifies two loci influencing age at menarche. Nature Genetics, 41(6), 648-650.

Pike, A., \& Kretschmer, T. (2009). Shared versus nonshared effects: parenting and children's adjustment. International Journal of Developmental Science, 3(2), 115-130.

Plomin, R. (1994). Genetics and experience: The interplay between nature and nurture. Sage series on individual differences and development, Vol. 6.

Polderman, T., Benyamin, B., Leeuw, C. A. de, Sullivan, P. F., Bochoven, A. van, Visscher, P. M., \& Posthuma, D. (2015). Meta-analysis of the heritability of human traits based on fifty years of twin studies. Nature Genetics, doi: 10.1038/ng.3285.

Price, A. L., Patterson, N. J., Plenge, R. M., Weinblatt, M. E., Shadick, N. A., \& Reich, D. (2006). Principal components analysis corrects for stratification in genome-wide association studies. Nature Genetics, 38(8), 904-909.

Purcell, S. M., Neale, B., Todd-Brown, K., Thomas, L., Ferreira, M. A. R., Bender, D., ... Daly, M. J. (2007). PLINK: a tool set for whole-genome association and population-based linkage analyses. The American Journal of Human Genetics, 81(3), 559-575.

Purcell, S. M., Wray, N. R., Stone, J. L., Visscher, P. M., O'Donovan, M. C., Sullivan, P. F., \& Sklar, P. (2009). Common polygenic variation contributes to risk of schizophrenia and bipolar disorder. Nature, 460(7256), 748-52. http: / / doi.org/10.1038/ nature08185

Rendall, M. S., Couet, C., Lappegard, T., Robert-Bobée, I., Rønsen, M., \& Smallwood, S. (2005). First births by age and education in Britain, France and Norway. Population Trends, (121), 27-34.

Rendall, M. S., \& Smallwood, S. (2003). Higher qualifications, first-birth timing and further childbearing in England and Wales. POPULATION TRENDS-LONDON-, 18-26.

Rietveld, C. A., Cesarini, D., Benjamin, D. J., Koellinger, P. D., Neve, J.-E. 
De, Tiemeier, H., ... Krueger, R. F. (2013). Molecular genetics and subjective well-being. Proceedings of the National Academy of Sciences, 110(24), 9692-9697.

Rietveld, C. A., Esko, T., Davies, G., Pers, T. H., Turley, P., Benyamin, B., \& Chabris, C. F. (2014). Common genetic variants associated with cognitive performance identified using the proxy-phenotype method. Proceedings of the National Academy of Sciences, 111(38), 13790-13794.

Rietveld, C. A., Medland, S. E., Derringer, J., Yang, J., Esko, T., Martin, N. W., ... Agrawal, A. (2013). GWAS of 126,559 Individuals Identifies Genetic Variants Associated with Educational Attainment. Science, 340(6139), 1467-1471.

Rijken, A. J., \& Liefbroer, A. C. (2009). Influences of the family of origin on the timing and quantum of fertility in the Netherlands. Population Studies, 63(1), 71-85.

Rindfuss, R. R., Guilkey, D., Morgan, S. P., Kravdal, O., \& Guzzo, K. B. (2007). Child care availability and first-birth timing in Norway. Demography, 44(2), 345-372. http: / / doi.org/10.1353/ dem.2007.0017

Rodgers, J. L., Bard, D. E., \& Miller, W. B. (2007). Multivariate cholesky models of human female fertility patterns in the NLSY. Behavior Genetics, 37, 345-361. http:/ / doi.org/10.1007/s10519-006-9137-9

Rodgers, J. L., Hughes, K., Kohler, H.-P., Christensen, K., Doughty, D., Rowe, D. C., \& Miller, W. B. (2001). Genetic influence helps explain variation in human fertility: Evidence from recent behavioral and molecular genetic studies. Current Directions in Psychological Science, 10(5), 184.

Rodgers, J. L., \& Kohler, H.-P. (2003). The biodemography of human reproduction and fertility. Springer Science \& Business Media.

Rodgers, J. L., Kohler, H.-P., Kyvik, K. O., \& Christensen, K. (2001). Behavior genetic modeling of human fertility: Findings from a contemporary Danish twin study. Demography, 38(1), 29-42.

Rodgers, J. L., Kohler, H.-P., McGue, M., Behrman, J. R., Petersen, I., Bingley, P., \& Christensen, K. (2008). Education and cognitive ability as direct, mediating, or spurious influences on female age at first birth: behavior genetic models fit to Danish twin data. AJS; American Journal of Sociology, 114(Suppl), S202.

Rosenzweig, M. R., \& Wolpin, K. I. (1980). Testing the Quantity-Quality Fertility Model: the Use of Twins as a Natural Experiment. 
Econometrica, 48(1), 227-240. http: / / doi.org/10.2307/1912026

Schmidt, L., Sobotka, T., Bentzen, J. G., \& Andersen, A. N. (2012). Demographic and medical consequences of the postponement of parenthood. Human Reproduction Update, 18(1), 29-43.

Scott, J. (2004). Family, gender, and educational attainment in Britain: A longitudinal study. Journal of Comparative Family Studies, 35(4).

Shanahan, M., \& Hofer, S. (2005). Social context in gene-environment interactions: Retrospect and prospect. The Journals of Gerontology Series B: Psychological Sciences and Social Sciences, 60(Special Issue 1), 65-76.

Skirbekk, V., Kohler, H. P., \& Prskawetz, A. (2006). The marginal effect of school leaving age on demographic events. A contribution to the discussion on causality. In S. Gustafsson \& A. Kalwij (Eds.), Education and postponement of maternity. Economic analyses for industrialized countries (pp. 65-85). Dordrecht, the Netherlands: Kluwer Academic Publishers.

Smith, H. L. (2003). Some Thoughts on Causation as It Relates to Demography and Population Studies. Population and Development Review, 29(3), 459-469. http://doi.org/10.1111/j.17284457.2003.00459.x

Snieder, H., MacGregor, A. J., \& Spector, T. D. (1998). Genes control the cessation of a woman's reproductive life: a twin study of hysterectomy and age at menopause. Journal of Clinical Endocrinology $\mathcal{E}$ Metabolism, 83(6), 1875-1880.

Snieder, H., Wang, X., \& MacGregor, A. J. (2010). Twin Methodology. In L. JohnWiley \& Sons (Ed.), . Chichester.

Snijders, T. A. B. (2011). Multilevel analysis. Berlin Heidelberg: Springer.

Sobotka, T. (2004). Is Lowest-Low Fertility in Europe Explained by the Postponement of Childbearing? Population and Development Review, 30(2), 195-220.

Speliotes, E., Willer, C., \& Berndt, S. (2010). Association analyses of 249,796 individuals reveal 18 new loci associated with body mass index. Nature ....

Stearns, S. C., Byars, S. G., Govindaraju, D. R., \& Ewbank, D. (2010). Measuring selection in contemporary human populations. Nature Reviews Genetics, 11(9), 611-622.

Steckel, R. H. (1995). Stature and the Standard of Living. Journal of 
Economic Literature, 33(4), 1903-1940. http:/ / doi.org/10.1111/j.14754991.1991.tb00335.x

Steenhof, L., \& Liefbroer, A. C. (2008). Intergenerational transmission of age at first birth in the Netherlands for birth cohorts born between 1935 and 1984: Evidence from municipal registers. Population Studies, 62(1), 69-84.

Stolk, L., Perry, J. R. B., Chasman, D. I., He, C., Mangino, M., Sulem, P., ... Ernst, F. (2012). Meta-analyses identify 13 loci associated with age at menopause and highlight DNA repair and immune pathways. Nature Genetics, 44(3), 260-268.

Stolk, L., Zhai, G., van Meurs, J. B. J., Verbiest, M. M. P. J., Visser, J. A., Estrada, K., ... Deloukas, P. (2009). Loci at chromosomes 13, 19 and 20 influence age at natural menopause. Nature Genetics, 41(6), 645647.

Stover, J. (1998). Revising the proximate determinants of fertility framework: what have we learned in the past 20 years? Studies in Family Planning, 29(3), 255-267. http:/ / doi.org/10.2307/ 172272

Stulp, G., Barrett, L., Tropf, F. C., \& Mills, M. C. (2015). Does natural selection favour taller stature among the tallest people on earth? Proceedings of the Royal Society of London B: Biological Sciences, 282(1806), 20150211.

Stulp, G., Verhulst, S., Pollet, T. V, \& Buunk, A. P. (2012). The effect of female height on reproductive success is negative in western populations, but more variable in non-western populations. American Journal of Human Biology, 24(4), 486-494.

Sulem, P., Gudbjartsson, D. F., Rafnar, T., Holm, H., Olafsdottir, E. J., Olafsdottir, G. H., ... Boyd, H. A. (2009). Genome-wide association study identifies sequence variants on $6 \mathrm{q} 21$ associated with age at menarche. Nature Genetics, 41(6), 734-738.

Taylor, A., Ware, J., \& Gage, S. (2015). Using molecular genetic information to infer causality in observational data: Mendelian randomisation. Current Opinion in Behavioral Sciences, 2, 39-45.

Thoday, J. M., Harrison, G. a., Brass, W., Fraser Roberts, J. a., Glass, D. V., Maynard Smith, J., ... Wrigley, E. a. (1970). The interrelation between genetics and the social sciences. Population Studies, 24(sup1), 49-54. http: / / doi.org/10.1080/00324728.1970.10404574

Thornton, A. (1980). The influence of first generation fertility and economic status on second generation fertility. Population and 
Environment, 3(1), 51-72.

Tobin, M. D., Sheehan, N. A., Scurrah, K. J., \& Burton, P. R. (2005). Adjusting for treatment effects in studies of quantitative traits: Antihypertensive therapy and systolic blood pressure. Statistics in Medicine, 24(19), 2911-2935. http:/ / doi.org/10.1002/sim.2165

Tropf, F. C., Barban, N., Mills, M. C., Snieder, H., \& Mandemakers, J. J. (2015). Genetic influence on age at first birth of female twins born in the UK, 1919-68. Population Studies, 69(2), 129-145.

Tropf, F. C., Stulp, G., Barban, N., Visscher, P., Yang, J., Snieder, H., \& Mills, M. C. (2015). Human fertility, molecular genetics, and natural selection in modern societies. PloS One, 10(6), e0126821.

Turkheimer, E. (2000). Three Laws of Behavior Genetics and What They Mean. Current Directions in Psychological Science, 9(5), 160-164. http: / / doi.org/10.1111/1467-8721.00084

Udry, J. R. (1996). Biosocial models of low-fertility societies. Population and Development Review, 22, 325-336.

van de Kaa, D. J. (1987). Europe's second demographic transition. Population Bulletin, 42(1), 1-59.

van den Oord, E. J. C. G., \& Snieder, H. (2002). Including measured genotypes in statistical models to study the interplay of multiple factors affecting complex traits. Behavior Genetics, 32(1), 1-22.

van Doorn, M., Pop, I., \& Wolbers, M. H. J. (2011). Intergenerational transmission of education across European countries and cohorts. European Societies, 13(1), 93-117.

Vaupel, J. W., Carey, J. R., Christensen, K., Johnson, T. E., Yashin, A. I., Holm, N. V, ... Curtsinger, J. W. (1998). Biodemographic Trajectories of Longevity. Science, 280(5365), 855-860. http: / / doi.org/10.1126/ science.280.5365.855

Verduijn, M., Siegerink, B., Jager, K. J., Zoccali, C., \& Dekker, F. W. (2010). Mendelian randomization: use of genetics to enable causal inference in observational studies. Nephrology Dialysis Transplantation, 25(5), 1394-1398.

Vinkhuyzen, A. A., Wray, N. R., Yang, J., Goddard, M. E., \& Visscher, P. M. (2013). Estimation and partition of heritability in human populations using whole-genome analysis methods. Annual Review of Genetics, 47, 75-95.

Visscher, P. M., Brown, M. A., McCarthy, M. I., \& Yang, J. (2012). Five 
years of GWAS discovery. The American Journal of Human Genetics, 90(1), 7-24.

Visscher, P. M., Hemani, G., Vinkhuyzen, A. A. E., Chen, G.-B., Lee, S. H., Wray, N. R., ... Yang, J. (2014). Statistical power to detect genetic (co) variance of complex traits using SNP data in unrelated samples. PLoS Genetics, 10(4), e1004269.

Visscher, P. M., Hill, W. G., \& Wray, N. R. (2008). Heritability in the genomics era-concepts and misconceptions. Nature Reviews Genetics, 9(4), 255-266.

Visscher, P. M., Yang, J., \& Goddard, M. E. (2010). A commentary on "common SNPs explain a large proportion of the heritability for human height"by Yang et al.(2010). Twin Research and Human Genetics, 13(06), 517-524.

Wachter, K. (1997). Between Zeus and the salmon: The biodemography of longevity.

Wachter, K. (2008). Biodemography comes of age. Demographic Research.

Wachter, K., \& Bulatao, R. (2003). Biodemography of fertility and family formation. OFFSPRING.

White, A., \& Folsom, A. (1996). Community surveillance of coronary heart disease in the Atherosclerosis Risk in Communities (ARIC) Study: methods and initial two years' experience. Journal of Clinical Epidemiology, 42(9), 223-233.

Yang, J., Bakshi, A., Zhu, Z., Hemani, G., Vinkhuyzen, A. A. E., Lee, S. H., ... Visscher, P. M. (2015). Genetic variance estimation with imputed variants finds negligible missing heritability for human height and body mass index. Nature Genetics, 47(10), 1114-1120. http: / / doi.org/10.1038/ng.3390

Yang, J., Benyamin, B., McEvoy, B. P., Gordon, S., Henders, A. K., Nyholt, D. R., ... Montgomery, G. W. (2010). Common SNPs explain a large proportion of the heritability for human height. Nature Genetics, 42(7), 565-569.

Yang, J., Lee, S. H., Goddard, M. E., \& Visscher, P. M. (2011). GCTA: a tool for genome-wide complex trait analysis. The American Journal of Human Genetics, 88(1), 76-82.

Yang, J., Manolio, T. A., Pasquale, L. R., Boerwinkle, E., Caporaso, N., Cunningham, J. M., ... Visscher, P. M. (2011). Genome partitioning of genetic variation for complex traits using common SNPs. Nature Genetics, 43(6), 519-25. http:/ / doi.org/10.1038/ng.823 
Zaitlen, N., Kraft, P., Patterson, N., Pasaniuc, B., Bhatia, G., Pollack, S., \& Price, A. L. (2013). Using extended genealogy to estimate components of heritability for 23 quantitative and dichotomous traits. PLoS Genetics, 9(5), e1003520.

Zhu, Z., Bakshi, A., Vinkhuyzen, A. A., Hemani, G., Lee, S. H., Nolte, I. M., \& Vliet-Ostaptchouk, J. V. van. (2015). Dominance genetic variation contributes little to the missing heritability for human complex traits. The American Journal of Human Genetics, 96(3), 377385.

Zietsch, B. P., Kuja-Halkola, R., Walum, H., \& Verweij, K. J. (2014). Perfect genetic correlation between number of offspring and grandoffspring in an industrialized human population. Proceedings of the National Academy of Sciences of the United States of America, 111(3), 1032-1036.

Zuk, O., \& Hechter, E. (2012). The mystery of missing heritability: Genetic interactions create phantom heritability. Proceedings of the National Academy of Science, 109(4), 1193-1198. 


\section{Supporting Information (SI)}

\section{SI 2-1 Local polynomial regression and non-parametric regression curve}

In order to fully explore the underlying trends of the latent factors, we applied a local polynomial regression to the factor estimates of each birth cohort using the Stata package Lowess. We first estimated the ACE-model of twin pairs of each birth year separately to produce time series of 46 estimates (birth cohorts born 1923-68) for the genetic influence, the shared environment, and the non-shared environment/measurement error. For cohorts born in the period 1919-22, the number of cases was too small to allow estimation.

In a second step, we partitioned the data into local areas around each birth year and, separately, fitted bivariate linear regressions of birth year onto the latent factors within each area. In practice, the choice of the local window of neighbourhood values included in the local regression is made by the researcher. It is a trade-off between bias in the estimation and the remaining variance between the local estimates. As default value of the nearest neighbourhood method, Lowess uses 80 of the observations in the time series for each local estimation. We chose a lower value to reduce bias and increase the visibility of changes in latent factors. We provided the results for 22 percent (equivalent to 10-year intervals) of the nearest neighbours, to show a good trade-off of reduced bias and acceptable variance in the factor trends. Note that the basic trends we discussed are not very sensitive and therefore the choice of this parameter was not critical for our substantive points. 
felix-tropf.com

SI 2-2 Scatterplot of estimated AFB for birth cohorts 1923-1968 from the UK and TwinsUK

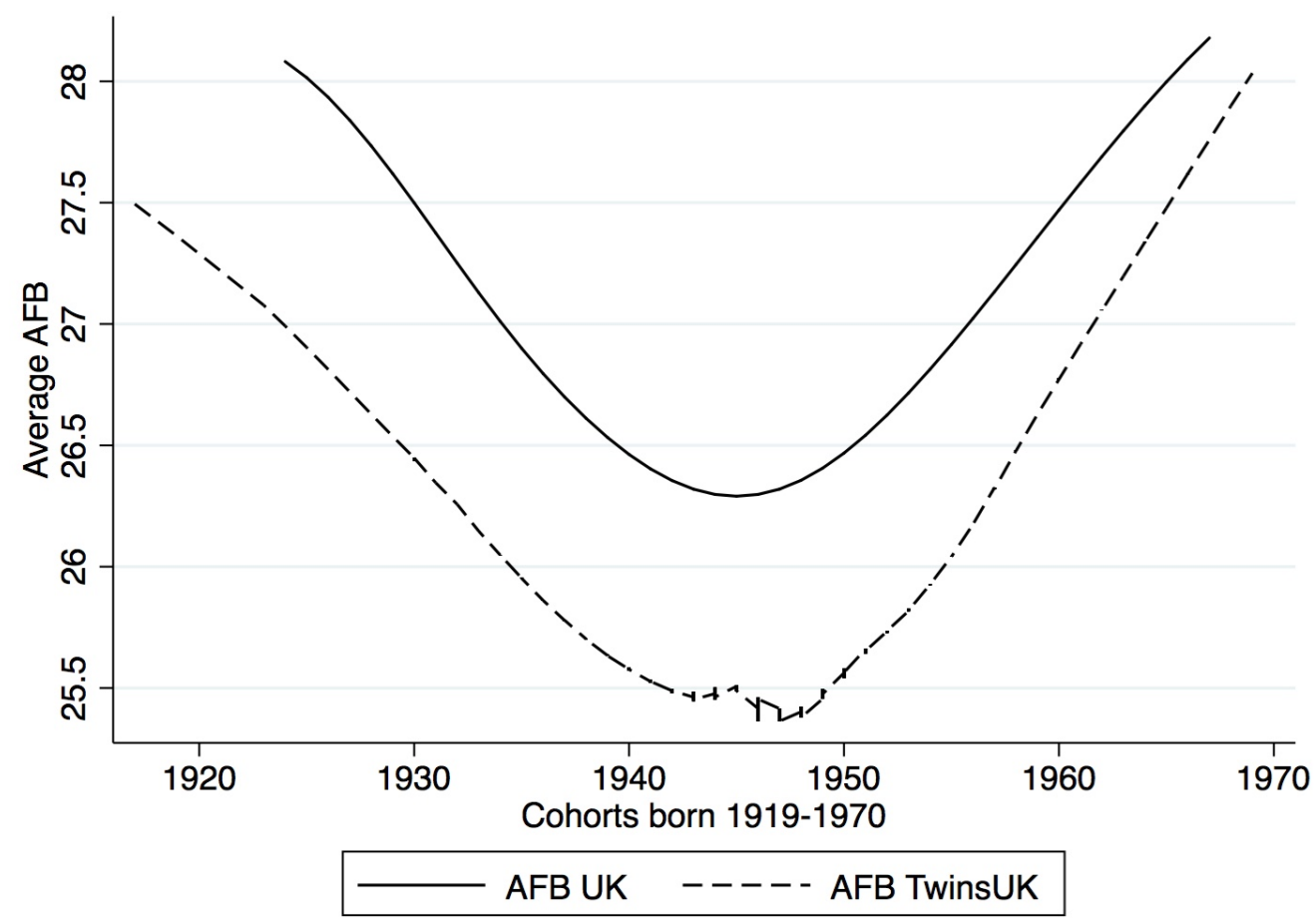

Notes: Estimated average AFB in the UK: Because, historically, official data on birth order have been only collected within marriage, these values are based on estimates from the Office for National Statistics. Estimates are based on average AFB per birth cohort and smoothed in a linear local regression (see Supporting Information 2-1 using 20 percent of the closest neighbourhood values.

Sources: TwinsUK and Office for National Statistics, Cohort fertility, Table 2 (Office National Statistics, 2013). 
SI 2-3 Smoothed scatterplot of local polynomial regressions of the birth year on heritability (y-axis 1 ) and the non-standardized genetic contribution (y-axis 2). UK Cohorts born 1923-68

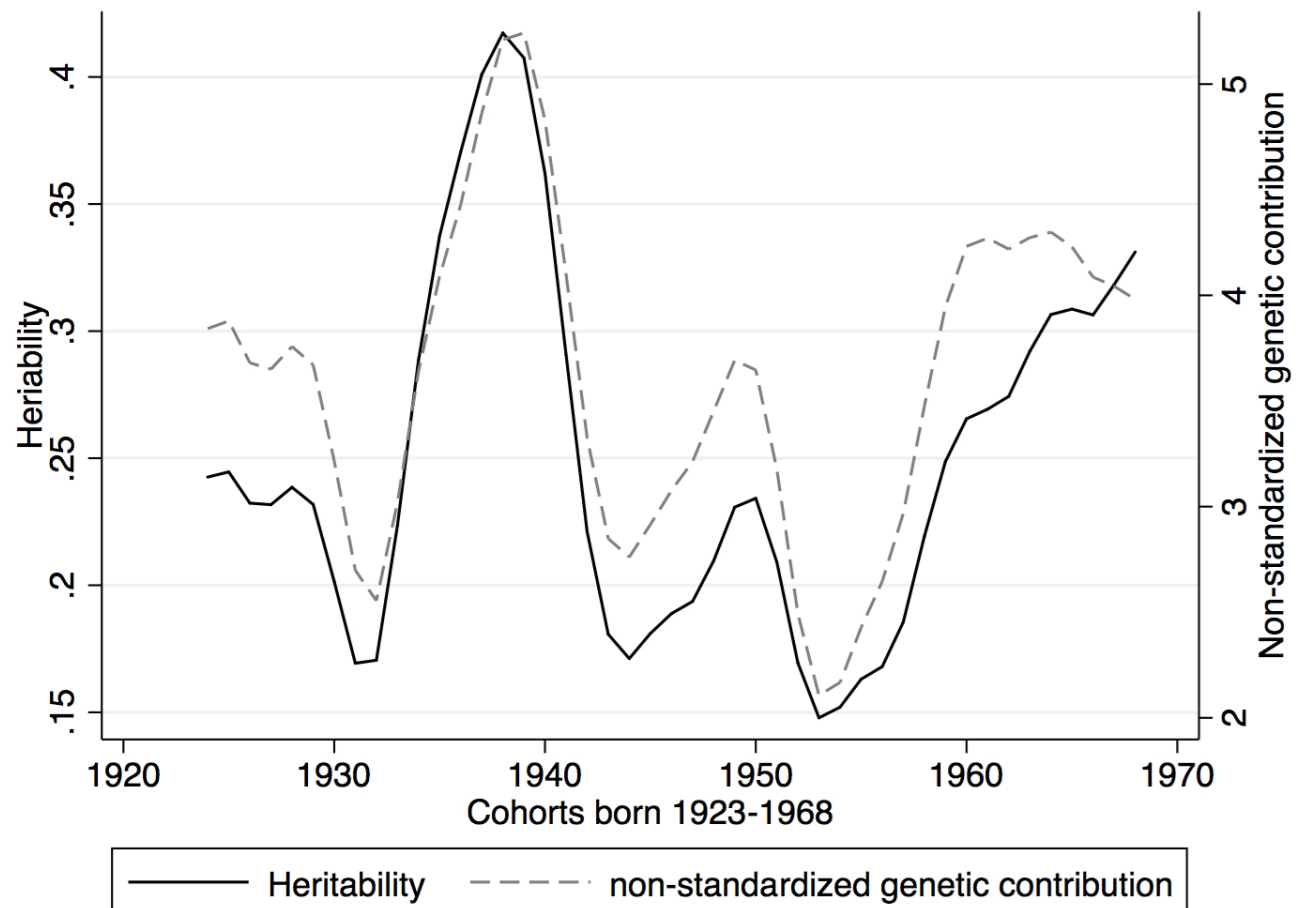

Notes: Smoothed results of local linear regressions based on the 10 closest birth cohorts. Confidence intervals for decades are based on estimates in Table 3. Cohorts born 1923-68.

Source: As for Table 2-1 


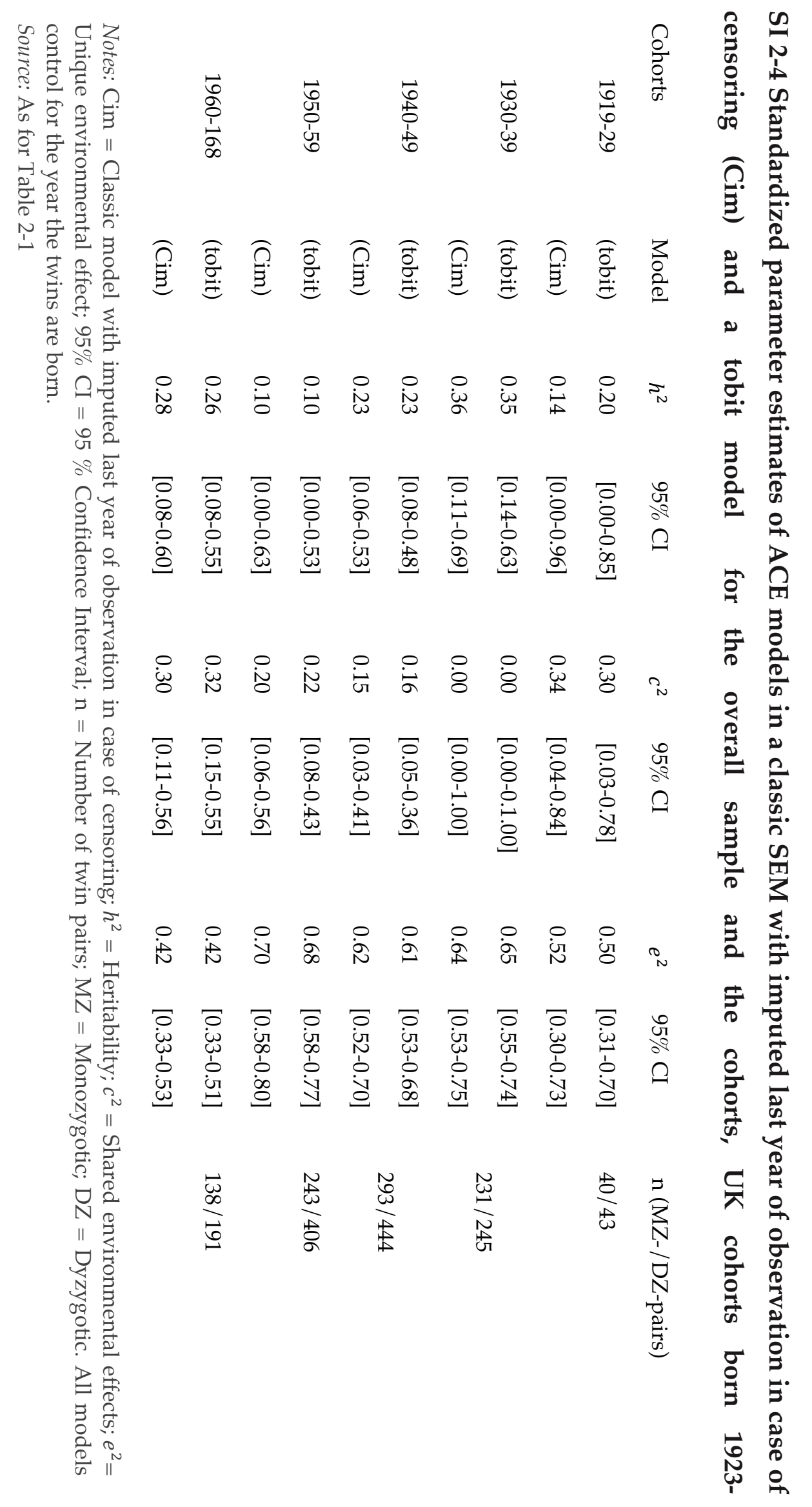




\section{Supporting Information}

\section{SI 3-1 Distributions of the dependent variables}

The graphs below show for the number of children ever born (SI 3-1a and the age at first birth (SI 3-1b) the distribution of the measured variables, the distribution of the Z-standardized variables and the log-transformed standardized variables (for the $\log$ transformation a constant has been added to the standardized value). SI 3-2 shows the results for all models on all phenotypes.

SI 3-1a Distribution of number of children ever born across the cohorts

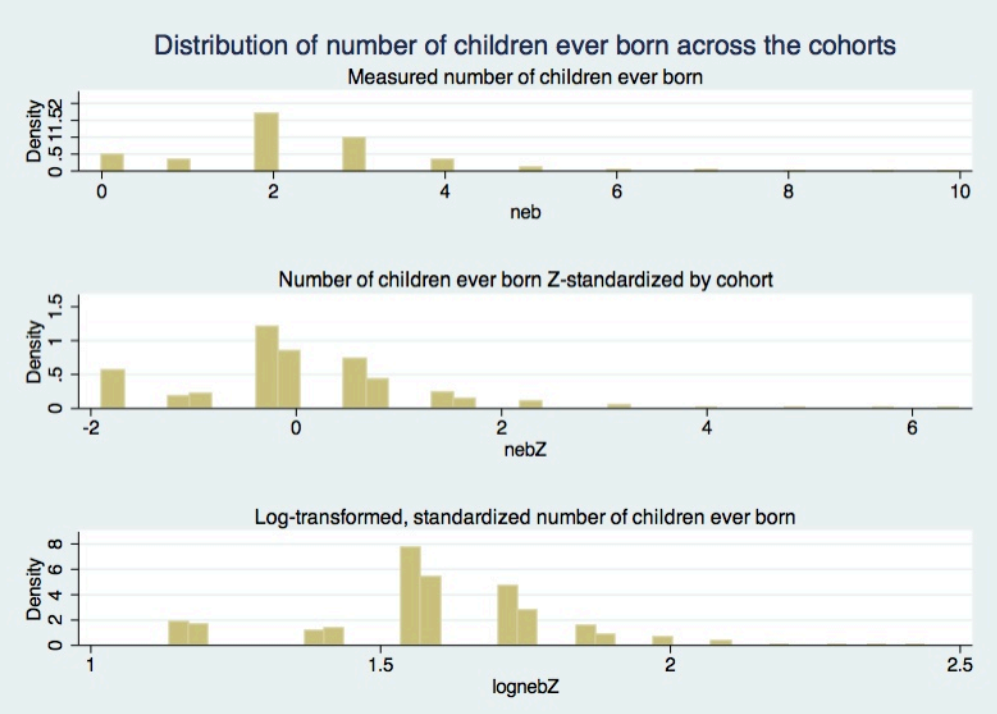

SI 3-1b Distribution of age at first birth across the cohorts

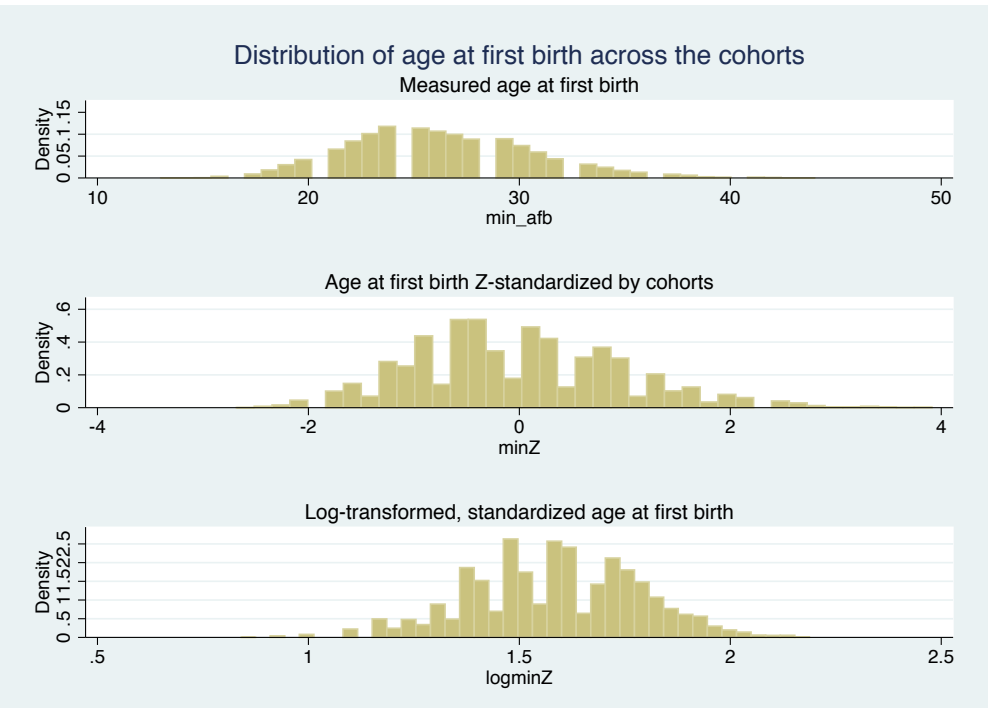


felix-tropf.com

SI 3-2 Estimates for NEB and AFB based on both the unstandardized measures and the transformations variables as seen in SI 3-1.

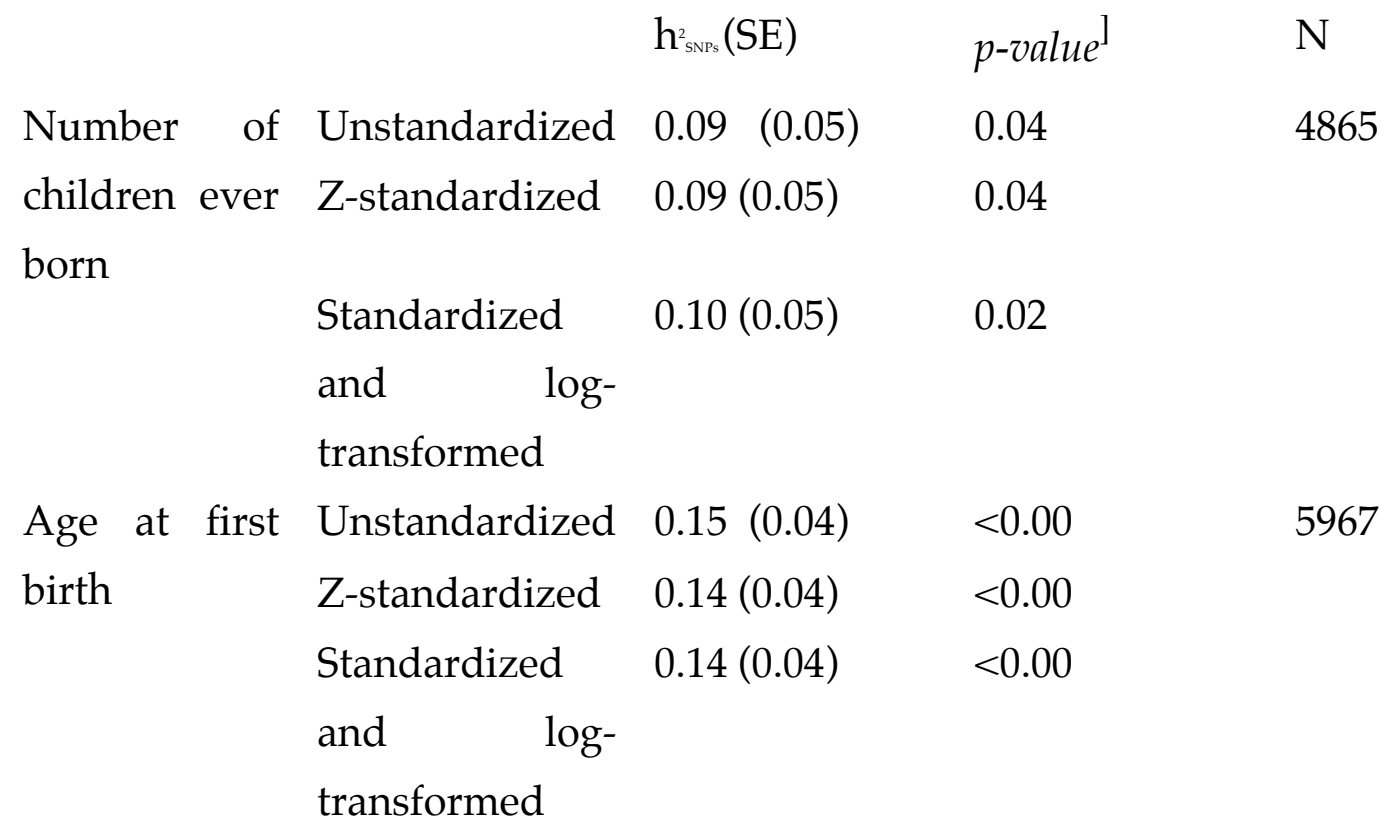

a: $p$-values are based on likelihood-ratio test compare to a reference model in which genetic effects are constrained to be 0 .

SI 3-3 Table. Genetic $\left(\sigma_{\mathrm{c}}^{2}\right)$ and environmental variance $\left(\sigma_{\mathrm{c}}^{2}\right)$ components and covariances $(\operatorname{Cov}(G), \operatorname{Cov}(E))$ of the bivariate model for $N E B(N=4865)$ and $A F B$ $(N=5967)$ in the pooled sample from the UK and the Netherlands using information from about 1 million SNPs

\begin{tabular}{|c|c|c|}
\hline \multirow[t]{2}{*}{ NEB } & $\sigma_{\mathrm{G}}{ }_{\mathrm{G}}(\mathrm{SE})$ & $0.004(0.002)$ \\
\hline & $\sigma_{\mathrm{e}}^{2}(\mathrm{SE})$ & $0.038(<0.002)$ \\
\hline \multirow[t]{4}{*}{ AFB } & $\sigma_{\mathrm{c}}{ }_{\mathrm{c}}(\mathrm{SE})$ & $0.144(0.043)$ \\
\hline & $\sigma_{\mathrm{e}}^{2}(\mathrm{SE})$ & $0.859(0.044)$ \\
\hline & $\sigma_{\mathrm{GNEB}, \mathrm{APB}}^{2}(\mathrm{SE})$ & $-0.014(0.007)$ \\
\hline & $\sigma_{\text {eNEB,ABB }}^{2}(S E)$ & $-0.064(0.008)$ \\
\hline
\end{tabular}

clxxiv 


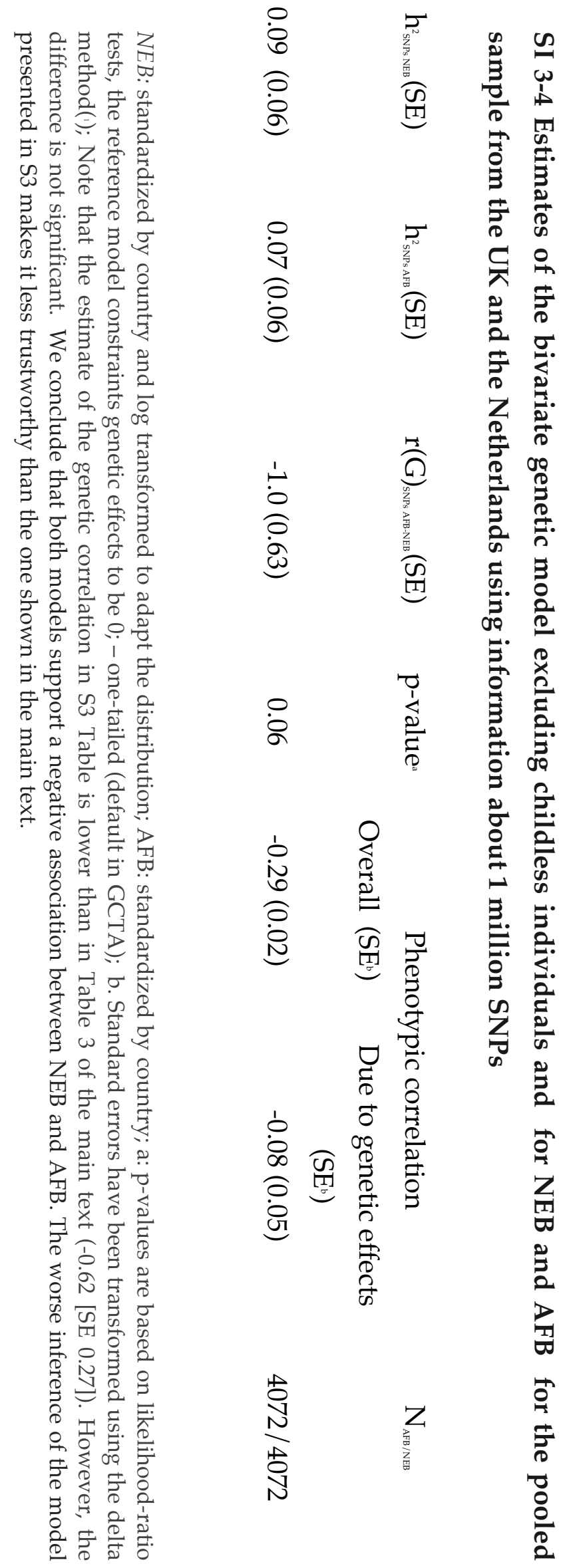




\section{SI 4-1 Summary statistics for women and men for all datasets separately and pooled}

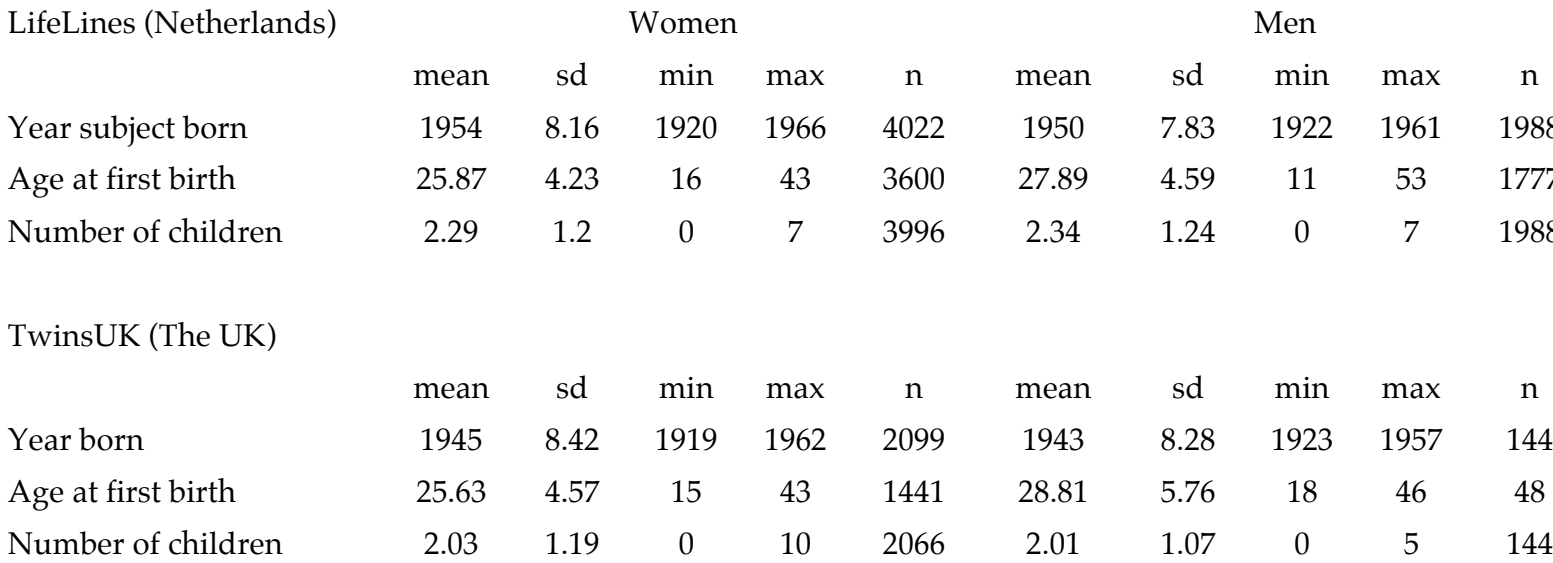

STR/SALT (Sweden)

$\begin{array}{lcccccccccc} & \text { mean } & \mathrm{sd} & \min & \max & \mathrm{n} & \text { mean } & \mathrm{sd} & \min & \max & \mathrm{n} \\ \text { Year born } & 1942 & 8.73 & 1912 & 1957 & 3122 & 1939 & 7.69 & 1911 & 1952 & 2918 \\ \text { Age at first birth } & 24.97 & 4.6 & 12 & 44 & 2720 & 27.57 & 4.79 & 13 & 49 & 2481 \\ \text { Number of children } & 2.02 & 1.19 & 0 & 9 & 3122 & 1.93 & 1.17 & 0 & 16 & 2918\end{array}$

EGCUT (Estonia)

Year born

$\begin{array}{cccccccccc}\text { mean } & \text { sd } & \min & \max & \mathrm{n} & \operatorname{mean} & \mathrm{sd} & \min & \max & \mathrm{n} \\ 1941 & 12.97 & 1905 & 1965 & 2217 & 1941 & 10.35 & 1910 & 1959 & 1503 \\ 24.55 & 4.46 & 15 & 46 & 1840 & 27.5 & 4.94 & 16 & 54 & 1275 \\ 2.01 & 1.24 & 0 & 11 & 2217 & 1.88 & 1.17 & 0 & 8 & 1503\end{array}$

QIMR (Australia)

Year born

Age at first birth

Number of children

$\begin{array}{cccccccccc}\text { mean } & \text { sd } & \min & \max & \mathrm{n} & \operatorname{mean} & \mathrm{sd} & \min & \max & \mathrm{n} \\ 1947 & 13.12 & 1903 & 1967 & 691 & 1946 & 10.96 & 1916 & 1962 & 477 \\ 26.65 & 4.77 & 17 & 41 & 527 & 29.75 & 5.58 & 18 & 49 & 400 \\ 3.3 & 1.68 & 0 & 11 & 691 & 3.37 & 1.67 & 0 & 11 & 476\end{array}$

HRS (USA)

Year born

$\begin{array}{cccccccccc}\text { mean } & \text { sd } & \min & \max & \mathrm{n} & \operatorname{mean} & \mathrm{sd} & \min & \max & \mathrm{n} \\ 1938 & 10.74 & 1905 & 1967 & 4873 & 1937 & 9.65 & 1907 & 1962 & 3462 \\ 2.6 & 1.65 & 0 & 13 & 4873 & 2.55 & 1.64 & 0 & 14 & 3460\end{array}$

ARIC (USA)

Year born

Number of children

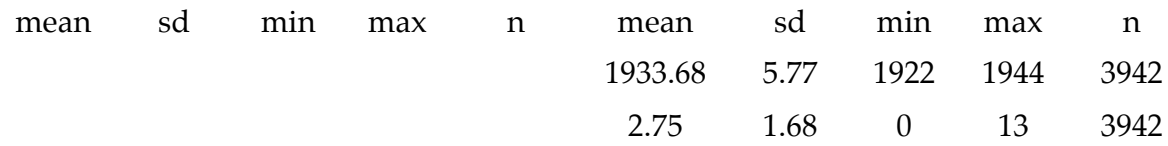

mean sd $\min \max \mathrm{n}$ mean $\mathrm{sd} \min \max \mathrm{n}$ 
Supporting Information

Continued SI 4-1

Pooled

$\begin{array}{lcccccccccc}\text { Year born } & 1942 & 11.62 & 1903 & 1967 & 20966 & 1941 & 10.13 & 1907 & 1962 & 10492 \\ \text { Age at first birth } & 25.4 & 4.49 & 12 & 46 & 10128 & 27.81 & 4.86 & 11 & 54 & 5981 \\ \text { Number of children } & 2.39 & 1.47 & 0 & 13 & 20907 & 2.27 & 1.42 & 0 & 16 & 10489\end{array}$

SI 4-2 Pearson's correlation between AFB and NEB for each dataset containing both phenotypes

Correlation Number of individuals

$\begin{array}{lcl}\text { LifeLines } & -0.24 & 5,351 \\ \text { TwinsUK } & -0.28 & 1,456 \\ \text { STR } & -0.30 & 5,201 \\ \text { QIMR } & -0.38 & 9,26 \\ \text { EGCUT } & -0.26 & 3,115\end{array}$


felix-tropf.com

SI 4-3 Comparison of total fertility rates across countries under study

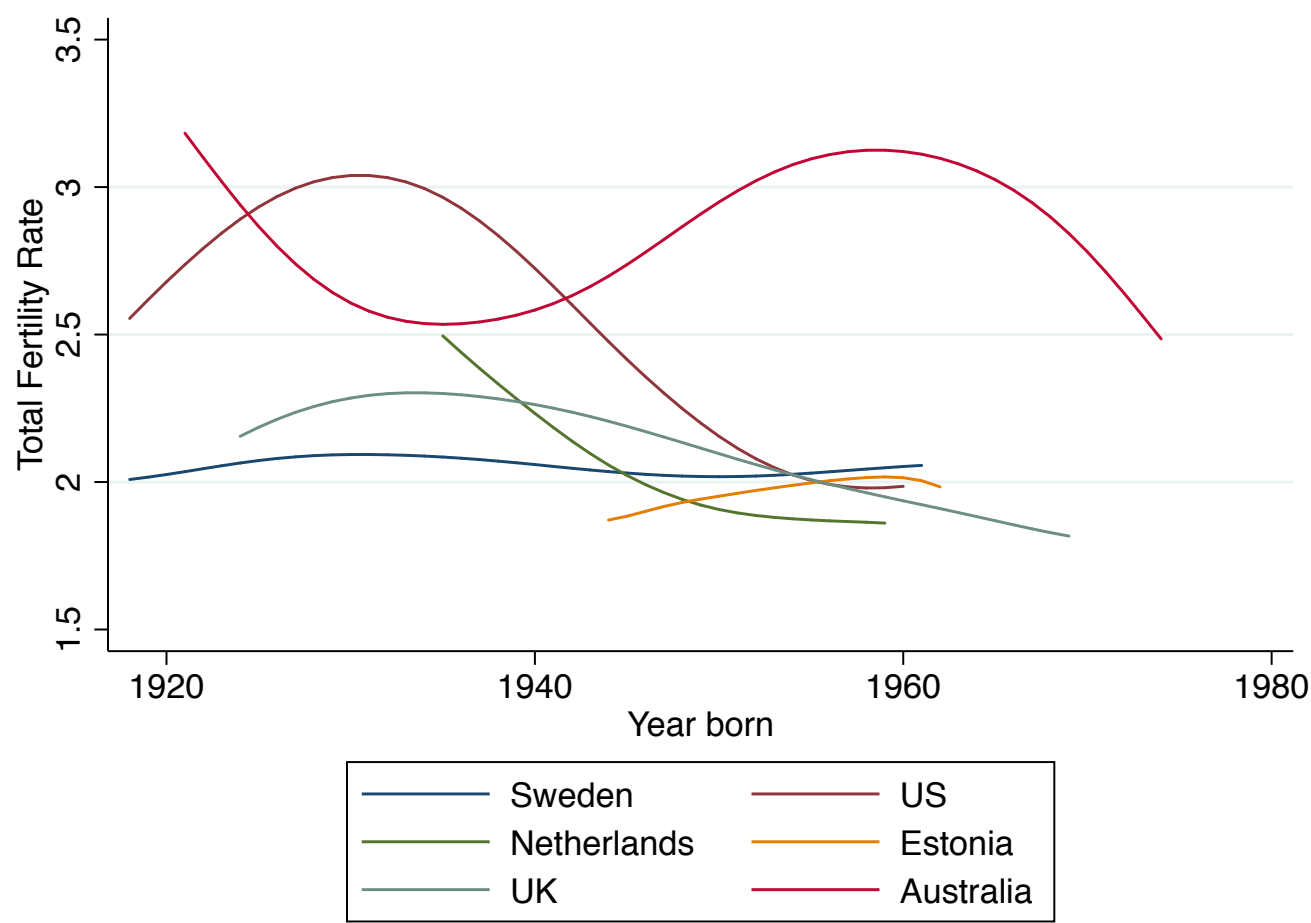

Note: Aggregated data have been obtained from the Human Fertility Database and the Human Fertility Collection (for Australia; for details see Material and Methods in the main text). The Total Fertility rate refers to the expected average number of children born to a woman. 
Supporting Information

SI 4-4 Observed mean age at first birth in QIMR data for Australia

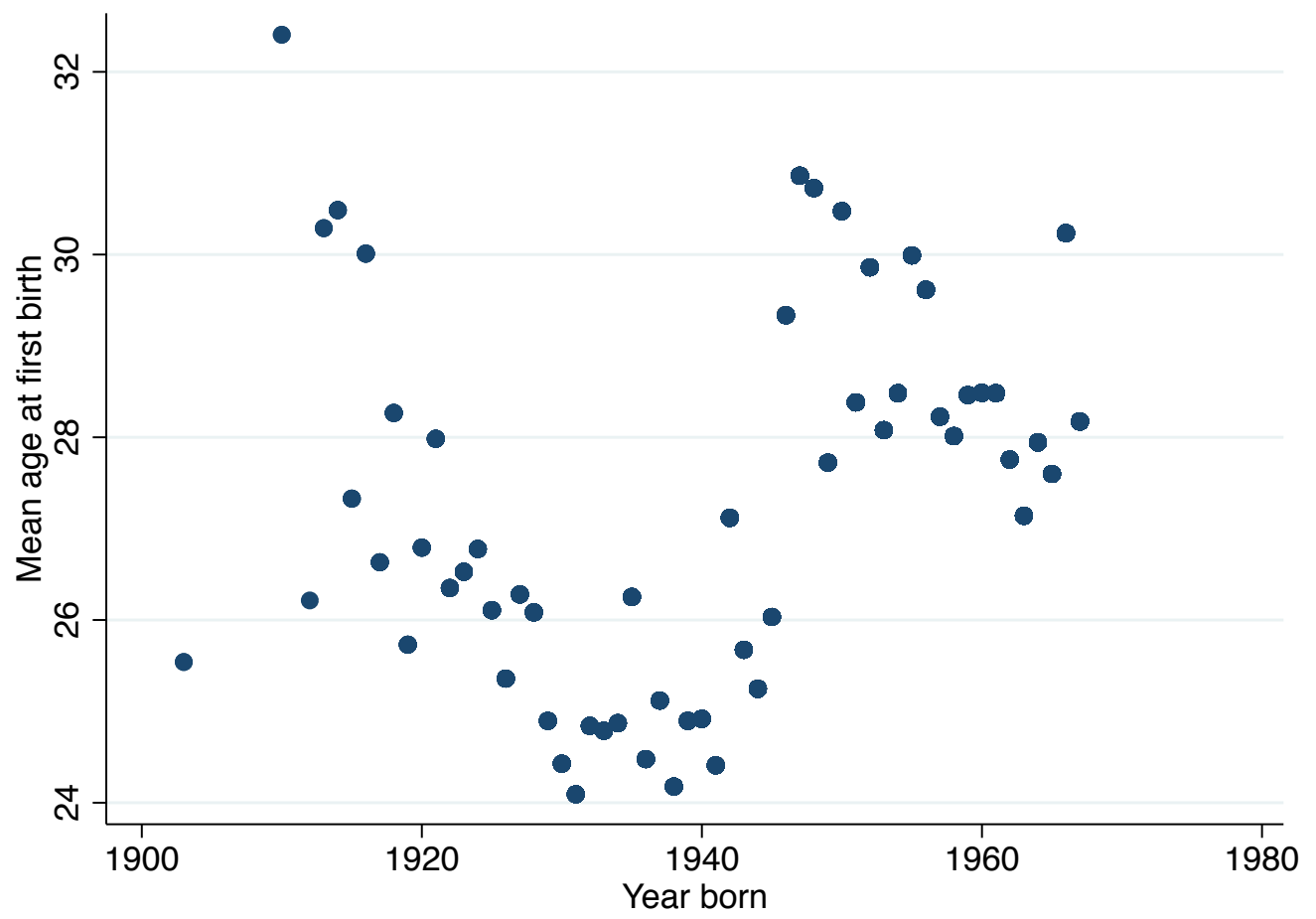

Note: For a description of the data source QIMR see Methods Section of Chapter 4 in the main text. 
felix-tropf.com

SI 4-5 Observed low point in the age at first birth on the population level by country - indicating the turning point in the fertility postponement trend

\begin{tabular}{llll} 
Country & Turning point & $\begin{array}{l}\text { Year of turning } \\
\text { point }\end{array}$ & Data source \\
\hline Netherlands & 26.39 & 1944 & HFD \\
United Kingdom & 25.75 & 1944 & Official Statistics \\
Sweden & 26.39 & 1943 & HFD \\
Australia & 24.78 & 1939 & QIMR \\
Estonia & 25.05 & 1962 & HFD/HFC \\
United States & 24.63 & 1940 & HFD
\end{tabular}

Notes: · See Material and Methods for details on data sources

SI 4-6. Overall SNP heritability estimated for Model specifications 1-4 for number of children ever born (NEB) and the age at first birth (AFB) as depicted in the main text Fig. 2.

NEB

$\begin{array}{lcccccccc}\text { Model } & 1 & 2 & 3 & 4 & 1 & 2 & 3 & 4 \\ \text { SUM }\left(\sigma^{2}\right) / \sigma_{P}^{2} & 0.038 & 0.15 & 0.097 & 0.22 & 0.05 & 0.13 & 0.094 & 0.19 \\ \text { SE } & (0.0097) & (0.022) & (0.012) & (0.026) & (0.02) & (0.034) & (0.024) & (0.039)\end{array}$


Supporting Information

SI 4-7 Sample sizes of datasets divided by demographic birth cohorts born before and after the onset of fertility postponement

\begin{tabular}{llll} 
Cohort & \multicolumn{1}{c}{$\begin{array}{c}\text { Before } \\
\text { postponement }\end{array}$} & $\begin{array}{c}\text { After } \\
\text { postponement }\end{array}$ & $\begin{array}{l}\text { Number } \\
\text { individuals }\end{array}$ \\
\hline LifeLines & 1,039 & 4,971 & 6,010 \\
TwinsUK & 992 & 1,251 & 2,243 \\
ARIC & 3,373 & 569 & 3,942 \\
HRS & 4,972 & 3,361 & 8,333 \\
STR & 3,561 & 2,479 & 6,040 \\
QIMR & 343 & 825 & 1,168 \\
EGCUT & 3,699 & 21 & 3,720
\end{tabular}

Notes: See Methods Secotion of chapter 4 for details on data sources. 
felix-tropf.com

SI 4-8 Heritability estimates of the gene environment interaction models for population and sex by number of children ever born (NEB) and age at first birth (AFB)

\begin{tabular}{|c|c|c|c|c|}
\hline \multirow[t]{3}{*}{ Model } & \multicolumn{4}{|c|}{$\mathrm{g} \times$ population $\mathrm{x}$ sex } \\
\hline & \multirow{2}{*}{$\begin{array}{c}\text { NEB } \\
\text { Estimate } \\
(\mathrm{SE})\end{array}$} & \multicolumn{3}{|c|}{ AFB } \\
\hline & & $\mathrm{p}$-value & $\begin{array}{c}\text { Estimate } \\
\text { (SE) }\end{array}$ & p-value \\
\hline$\sigma_{g}^{2} / \sigma_{P}^{2}$ & $\begin{array}{c}0.000001 \\
(0.015)\end{array}$ & 0.5 & $\begin{array}{c}0.012 \\
(0.032)\end{array}$ & 0.35 \\
\hline$\sigma_{g x p}^{2} / \sigma_{P}^{2}$ & $\begin{array}{c}0.11 \\
(0.036)\end{array}$ & 0.0010 & $\begin{array}{c}0.12 \\
(0.059)\end{array}$ & 0.013 \\
\hline$\sigma_{g x s}^{2} / \sigma_{P}^{2}$ & $\begin{array}{c}0.019 \\
(0.020)\end{array}$ & 0.18 & $\begin{array}{c}0.021 \\
(0.043)\end{array}$ & 0.31 \\
\hline$\sigma_{g x p x s}^{2} / \sigma_{P}^{2}$ & $\begin{array}{c}0.051 \\
(0.046)\end{array}$ & 0.093 & $\begin{array}{c}0.000001 \\
(0.079)\end{array}$ & 0.5 \\
\hline $\mathrm{N}$ & 31,3 & & 16,1 & \\
\hline
\end{tabular}

Note: $\mathrm{NEB}=$ number of children ever born, $\mathrm{AFB}=$ age at first birth, $\sigma_{g}^{2} / \sigma_{P}^{2}=$ proportion of observed variance in the outcome associated with genetic variance across populations and sexes, $\sigma_{g x p}^{2} / \sigma_{P}^{2}=$ proportion of observed variance in the outcomes associated with additional genetic variance within populations, $\sigma_{g x s}^{2} / \sigma_{P}^{2}=$ proportion of observed variance associated with additional genetic variance within sexes, $\sigma_{g x p x s}^{2} / \sigma_{P}^{2}=$ proportion of observed variance associated with additional genetic variance within populations and sexes, p-values are based on likelihood-ratio test comparing the full model with the model with one constraining the particular effect to be zero. All analyses include the first 20 Principal Components, and outcomes are standardized for sex, birth year and country. The model extends Model specification 2 (see Material \& Methods) with $\boldsymbol{A}_{\boldsymbol{g x s}}$, which is a matrix only with values for pairs of individuals within the same sex and $\boldsymbol{A}_{\text {gxpxs }}$, which is a matrix only with values for pairs of individuals with both the same sex and from the same population (following the same systematic as the extension of Model 3 to Model 4). 


\section{SI 5-1. (Stratified) Cox regression models}

In order to draw more general conclusions about the effect of education on AFB we use Cox regression models that also incorporate censored cases, namely women who have not conceived a child at the last time of observation (right-censored) as well as those younger than 40 years old at last observation (right-censored women and those with children) $(N=4,398)$. See SI 5-2 for descriptive statistics for this larger sample of women. For childless women we used the last age at observation as the censoring age. The Cox model estimates $\lambda_{i}(t)$ as the instantaneous risk of an individual $\mathrm{i}$ at time $\mathrm{t}$ to have a child in case it she did not have a child. Analogous to the linear regression model from equation (1) (main text), we fit the following Cox regression model:

$$
\begin{aligned}
& \lambda_{i}(t)=\lambda_{0}(t) \exp \left(\beta_{1}\left(\text { education }_{i}\right)+\beta_{2}\left(\text { birth year }_{i}\right)+\beta_{3}\left({\text { birth } \left.\text { year }_{i}^{2}\right)}\right.\right. \\
&\left.+\beta_{3}\left(\text { zygosity }_{i}\right)\right)
\end{aligned}
$$

where $\lambda_{0}(t)$ represents an arbitrary baseline hazard and $\exp \left(\beta^{\prime}\right)$ the regression parameters. In the standard exponential form, covariates enter linearly. We will present the coefficients as the relative change in the hazard, namely the hazard ratio of a coefficient $\exp \left(\beta_{1}-1\right)$.

For the within-family models we estimate Cox regression models by stratifying by family. This is analogous to a fixed effects model for censored data (Allison \& Christakis, 2006):

$$
\lambda_{i g}(t)=\lambda_{0 g}(t) \exp \left(\beta_{1}\left(\text { education }_{i}\right)\right)
$$

where $\lambda_{0 g}(t)$ represents an arbitrary family-specific baseline hazard. Note that variables that are constant within families are subsumed by the family stratum and therefore drop from the equation. SI 5-3 shows the results analogous to the OLS models in Table 2 in the main text. Results are well in line with the findings from the OLS. 


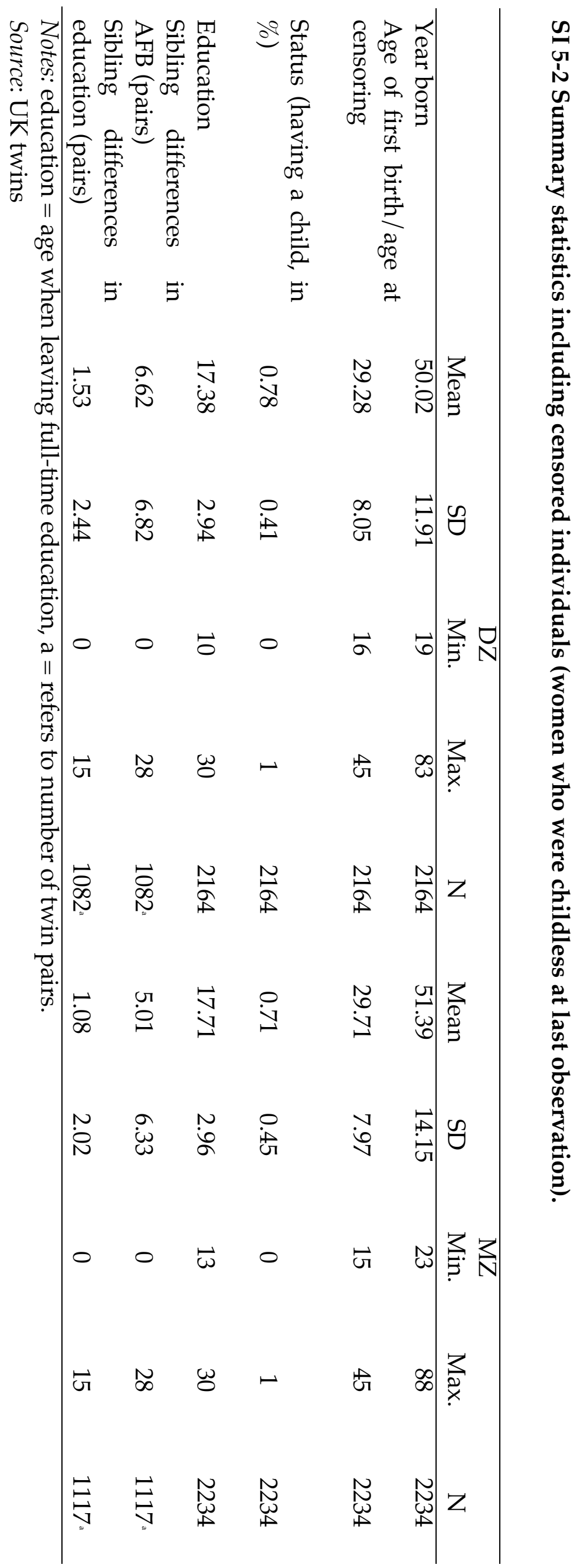


SI 5-3 Cox- and stratified cox-regression for monozygotic (MZ) and dizygotic (DZ) female UK twins, born 1919-88. (analogous to Table 2 in main text)

\begin{tabular}{|c|c|c|c|c|c|c|}
\hline & \multicolumn{2}{|c|}{ Full sample } & \multicolumn{2}{|c|}{ MZ only } & \multicolumn{2}{|c|}{ DZ only } \\
\hline Model & $\begin{array}{c}1 \\
\text { Cox }\end{array}$ & $\begin{array}{c}2 \\
\text { Stratified }\end{array}$ & $\begin{array}{c}3 \\
\text { Cox }\end{array}$ & $\begin{array}{c}4 \\
\text { Stratified }\end{array}$ & $\begin{array}{c}5 \\
\text { Cox }\end{array}$ & $\begin{array}{c}6 \\
\text { Stratified }\end{array}$ \\
\hline Education & $\begin{array}{c}-0.099^{* * *} \\
(0.008)\end{array}$ & $\begin{array}{c}-0.039^{*} \\
(0.018)\end{array}$ & $\begin{array}{c}-0.102^{* \star *} \\
(0.012)\end{array}$ & $\begin{array}{c}-0.033 \\
(0.029)\end{array}$ & $\begin{array}{c}-0.096^{\star \star \star} \\
(0.011)\end{array}$ & $\begin{array}{c}-0.042 \\
(0.023)\end{array}$ \\
\hline Year Born & $\begin{array}{c}0.130^{* * *} \\
(0.012)\end{array}$ & & $\begin{array}{c}0.131^{* * *} \\
(0.017)\end{array}$ & & $\begin{array}{c}0.128^{* * *} \\
(0.018)\end{array}$ & \\
\hline Year born squared & $\begin{array}{c}-0.002^{* * *} \\
(0.00)\end{array}$ & & $\begin{array}{c}-0.002^{* * *} \\
(0.00)\end{array}$ & & $\begin{array}{c}-0.001^{* * *} \\
(0.000)\end{array}$ & \\
\hline Zygosity & $\begin{array}{l}-0.081 \\
(0.079)\end{array}$ & & & & & \\
\hline Observations & 4398 & 4398 & 2234 & 2234 & 2164 & 2164 \\
\hline $\begin{array}{l}\text { Notes: Education }= \\
1900, \text { Zygosity }=0 \\
\text { independence of th } \\
{ }^{*} \mathrm{p}<0.05,{ }^{* *} \mathrm{p}<0.01, \\
\text { Source: TwinsUK, }\end{array}$ & $\begin{array}{l}\text { ge when } \\
\text { for DZ a } \\
\text { ss, } \\
{ }^{*} \text { p }<0.001 \\
\text { n calcula }\end{array}$ & $\begin{array}{l}\text { leaving ful } \\
\text { d } 1 \text { for MZ } \\
\text { one-sided } \\
\text { ions }\end{array}$ & $\begin{array}{l}\text {-time edu } \\
\text { twins, st }\end{array}$ & $\begin{array}{l}\text { cation, ye } \\
\text { ndard err }\end{array}$ & $\begin{array}{l}\text { born }=y \\
\text { rs correcte }\end{array}$ & $\begin{array}{l}\text { ear born - } \\
\text { d for non- }\end{array}$ \\
\hline
\end{tabular}


felix-tropf.com

SI 5-4 Linear OLS and fixed-effects regression on $M Z$ and DZ female UK twins, born 1919-44.

\begin{tabular}{lcc}
\hline & \multicolumn{2}{c}{ Full sample } \\
\hline & \multicolumn{1}{c}{$\begin{array}{cc}1 \\
\text { OLS }^{\mathrm{a}}\end{array}$} & $\mathrm{FE}$ \\
\cline { 2 - 3 } Education & $\begin{array}{c}0.38^{* * *} \\
(0.06)\end{array}$ & $\begin{array}{c}0.11 \\
(0.14)\end{array}$
\end{tabular}

Cohort (if birth year $<1945=0$; else 1) $\quad-1.97$

Education ${ }^{*}$ Cohort

$\begin{array}{ll}0.11 & 0.004 \\ (0.08) & (0.03)\end{array}$

Zygosity

0.15

(0.38)

Constant

$19.37^{* * *} \quad 24.14^{* * *}$

(0.99)

(1.13)

Observations

2752

2752

Notes: Education $=$ age when leaving full-time education, year born = year born -1900 , Zygosity $=0.5$ for DZ and 1 for MZ twins, $\mathrm{a}=$ OLS standard errors corrected for non-independence of twins,

${ }^{*} \mathrm{p}<0.05,{ }^{* *} \mathrm{p}<0.01,{ }^{* * *} \mathrm{p}<0.001$

Source: TwinsUK, own calculations

clxxxvi 


\section{SI 5-5 Correlation, covariance and covariance components in biometric models}

SI 5-4 shows the unstandardized estimates from the ACE-beta model. Figure 5-3 in the main text shows the transformed estimates into standardized variance components, the correlation between the variance components and we mention in the text the decomposition of the observed correlation between education and age at first birth into the contribution by additive genetic, shared environmental and a causal effect from the ACE-beta model. Here we briefly demonstrate the derivation of these quantities.

\section{Variance components}

ACE-beta model (Kohler et al., 2011)

In behavioral genetics it is standard to present the estimates of the latent factors as variance components. Additive genetic influences on education are represented as genetic variance in education $a_{e d u}^{2}$ over the overall variance in education (caused by all underlying factors $a_{e d u}^{2}+c_{e d u}^{2}+e_{e d u}^{2}$ ).

From SI 5-4: $h_{e d u}^{2}=\left(a_{e d u}^{2} /\left(a_{e d u}^{2}+c_{e d u}^{2}+e_{e d u}^{2}\right)=1.77^{2} /\left(1.77^{2}+1.24^{2}+1.44^{2}\right)=0.46\right.$

For heritability of age at first birth the influence of the unique environment is replaced by a direct link between education and age at first birth. It therefore consists of three elements: i) direct genetic influences unique to age at first birth, ii) direct genetic influences which are shared between education and age at first birth iii) indirect genetic influences which operate via education and can be estimate by

$$
h_{a f b}^{2}=\frac{\beta^{2} a_{e d u}^{2}+2 \beta a_{e d u, a f b}+a_{e d u, a f b}^{2}+a_{a f b, a f b}^{2}}{\sigma_{a f b}^{2}}
$$

where $\sigma_{a f b}^{2}=\beta^{2}\left(\sigma_{e d u}^{2}\right)^{2}+2 \beta\left(a_{e d u, a f b}+c_{e d u, a f b}\right)+\left(a_{a f b, a f b}^{2}+a_{e d u, a f b}^{2}+c_{a f b, a f b}^{2}+\right.$ $\left.c_{e d u, a f b}^{2}+e_{a f b, a f b}^{2}\right)$.

\section{Correlation}

The correlation of the latent factors in the model, for example for the shared environmental effects (parallel for genetic effects) is: 
felix-tropf.com

$$
\mathrm{r}(\mathrm{c})=\frac{c_{e d u} * c_{e d u, a f b}}{\sqrt{c_{e d u}^{2}\left(c_{e d u, a f b}^{2}+c_{a f b, a f b}^{2}\right)}}
$$

A correlation of $r(c)=1$ means that all shared environmental effects for fertility are associated with shared environmental effect for education, while a correlation of $r(c)=0$ means that both effects are independent.

\section{Covariance componance}

The overall covariance between education and age at first birth can be decomposed in parts which are due to genetic, shared environmental effects and due to the direct/causal effect. This is achieved by calculating the ratio of the respective covariance over the overall variance - the sum of the three components:

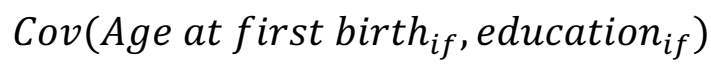

$$
=a_{e d u} a_{e d u, a f b}+c_{e d u} c_{e d u, a f b}+\beta \sigma^{2}\left(\text { education }_{i j}\right)
$$

For example, the contribution of the genetic covariance to the overall covariance is:

$$
\frac{a_{e d u} a_{e d u, a f b}}{a_{e d u} a_{e d u, a f b}+c_{e d u} c_{e d u, a f b}+\beta \sigma^{2}\left(\text { education }_{i j}\right)}=
$$

$$
\frac{1.77 * 0.37}{1.77 * 0.37+1.24 * 1.48+0.07 * 6.74}=0.22
$$

This means that 22 percent of the correlation between education and age at first birth is due to shared genetic effects. The respective values for the shared environment of the siblings 62 percent and for the causal effect 16 percent. 
SI 5-6 Unstandardized estimates of the ACE-beta models. $N$ observations $=2,752$; $N$ twins $=1,376$.

\begin{tabular}{lll}
\hline Model & \multicolumn{2}{c}{ ACE-beta } \\
& estimate & se \\
\hline education & $1.77^{* * *}$ & .12 \\
$a$ & $1.24^{* * *}$ & .15 \\
$c$ & $1.44^{* * *}$ & .04 \\
$e$ & & \\
age at first birth & $2.53^{* * *}$ & .22 \\
$a$ & 0.00 & .94 \\
$c$ & $3.42^{* * *}$ & .09 \\
$e$ & & \\
cross-trait & & .39 \\
effects & 0.37 & .31 \\
$a$ & $1.48^{* * *}$ & - \\
$c$ & - & .09 \\
$e$ & 0.07 & \\
Beta & & \\
\hline
\end{tabular}

Notes: * $\mathrm{p}<0.05,{ }^{* *} \mathrm{p}<0.01,{ }^{* *} \mathrm{p}<0.005$

Source: UKtwins, own calculations 


\section{Funding}

Research

The research leading to these results was funded by the Dutch Science Foundation (VIDI Innovation Grant 452-10-012 to M. Mills, the European Research Council (ERC) Consolidator Grant SOCIOGENOME (615603, www.sociogenome.com) and Economic \& Social Research Council (ESRC) UK, National Centre for Research Methods (NCRM) SOCGEN grant (www.ncrm.ac.uk/research/SoCGEN/). Felix C. Tropf additionally received funding from sciencestarter.de for the research visit to the University of Queensland.

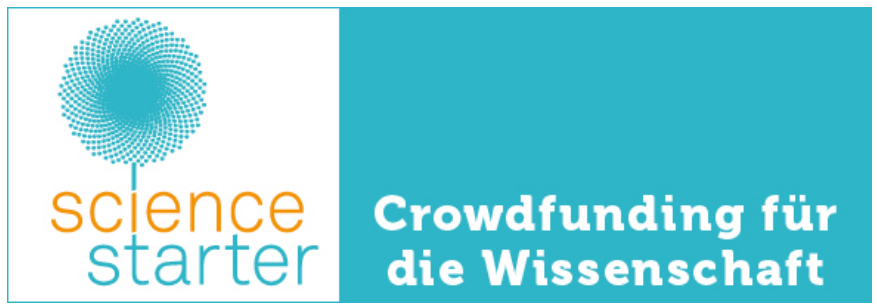

Data

The Atherosclerosis Risk in Communities Study (ARIC) is carried out as a collaborative study supported by National Heart, Lung, and Blood Institute contracts HHSN268201100005C, HHSN268201100006C, HHSN268201100007C, HSN268201100008C, HHSN268201100009C, HHSN268201100010C, HSN268201100011C, and HHSN268201100012C). The authors thank the staff and participants of the ARIC study for their important contributions. Funding for CARe genotyping was provided by NHLBI Contract N01-HC-65226. Funding for GENEVA was provided by National Human Genome Research Institute grant U01HG004402 (E. Boerwinkle).

EGCUT (Estonian Genome Center, University of Tartu) - This study was supported by the Estonian Research Council (grant IUT20-60), the Development Fund of the University of Tartu (grant SP1GVARENG), EU structural support 
through Archimedes Foundation, grant no: 3.2.1001.11-0033, EU 7FP grant 278913, and H2020 grants 633589, 676550, 654248.

HRS is supported by the National Institute on Aging (NIA U01AG009740). The genotyping was funded separately by the National Institute on Aging (RC2 AG036495, RC4 AG039029). Genotyping was conducted by the NIH Center for Inherited Disease Research (CIDR) at Johns Hopkins University. Genotyping was performed by the Genetics Coordinating Center at the University of Washington. Genotype data can be accessed via the database of Genotypes and Phenotypes (dbGaP, http://www.ncbi.nlm.nih.gov/gap, accession number phs000428.v1.p1). Researchers who wish to link genetic data with other HRS measures that are not in $\mathrm{dbGaP}$, such as educational attainment, must apply for access from HRS. See the HRS website (http: / / hrsonline.isr.umich.edu/gwas) for details.

The LifeLines Cohort Study, and generation and management of GWAS genotype data for the LifeLines Cohort Study is supported by the Netherlands Organization of Scientific Research NWO (grant 175.010.2007.006), the Economic Structure Enhancing Fund (FES) of the Dutch government, the Ministry of Economic Affairs, the Ministry of Education, Culture and Science, the Ministry for Health, Welfare and Sports, the Northern Netherlands Collaboration of Provinces (SNN), the Province of Groningen, University Medical Center Groningen, the University of Groningen, Dutch Kidney Foundation and Dutch Diabetes Research Foundation.

QIMR - Funding was provided by the Australian National Health and Medical Research Council (241944, 339462, 389927, 389875, 389891, 389892, 389938, 442915, 442981, 496739, 552485, 552498), the Australian Research Council (A7960034, A79906588, A79801419, DP0770096, DP0212016, DP0343921), the FP- 5 GenomEUtwin Project (QLG2-CT-2002-01254), and the U.S. National Institutes of Health (NIH grants AA07535, AA10248, AA13320, AA13321, AA13326, AA14041, DA12854, MH66206). A portion of the genotyping was carried out at the Center for Inherited Disease Research, Baltimore (CIDR), through an access award to the authors' late colleague Dr. Richard Todd (Psychiatry, Washington University School of Medicine, St Louis). Imputation was carried out on the Genetic Cluster Computer, which is financially supported by the Netherlands Scientific Organization (NWO 480-05-003). N.W.H.M was supported by a PhD scholarship from the ANZ trust. S.E.M., is supported by the Australian Research Council (ARC) Fellowship Scheme. 
felix-tropf.com

Dale R. Nyholt is supported by the Australian Research Council (ARC) Future Fellowship (FT0991022) and National Health and Medical Research Council (NHMRC) Research Fellowship (APP0613674) Schemes. The funders had no role in study design, data collection and analysis, decision to publish, or preparation of the manuscript. Researchers interested in using QIMR data can contact Nick Martin (Nick.Martin@qimrberghofer.edu.au) and Sarah Medland (medlandse@gmail.com).

STR (Swedish Twin Registry) - The Jan Wallander and Tom Hedelius Foundation (P2012- 0002:1), the Ragnar Söderberg Foundation (E9/11), The Swedish Research Council (421-2013- 1061), the Ministry for Higher Education, The Swedish Research Council (M-2205-1112), GenomEUtwin (EU/QLRT-2001-01254; QLG2-CT2002-01254), NIH DK U01-066134, The Swedish Foundation for Strategic Research (SSF). Researchers interested in using STR data must obtain approval from the Swedish Ethical Review Board and from the Steering Committee of the Swedish Twin Registry. Researchers using the data are required to follow the terms of an Assistance Agreement containing a number of clauses designed to ensure protection of privacy and compliance with relevant laws. For Further information, contact Patrik Magnusson (Patrik.magnusson@ki.se).

The TwinsUK study was funded by the Wellcome Trust; European Community's Seventh Framework Programme (FP7/2007-2013). The study also received support from the National Institute for Health Research (NIHR)- funded BioResource, Clinical Research Facility and Biomedical Research Centre based at Guy's and St Thomas' NHS Foundation Trust in partnership with King's College London. SNP Genotyping was performed by The Wellcome Trust Sanger Institute and National Eye Institute via NIH/CIDR. 


\section{Data availability statement}

Data were collected and maintained by third parties. For ethical and legal reasons we are not allowed to distribute them. However, it is possible to contact the studies directly to request access to data as specified on their respective websites: The TwinsUK data is available on request by contacting the Twin Research Unit at www.twinsuk.ac.uk/data-access/submission-procedure. The LifeLines data is available by contacting the LifeLines Research Office (LLscience@umcg.nl)(https: / / www.lifelines.nl/lifelines-research/access-

tolifelines/application-process). Researchers interested in using STR data can contact Patrik Magnusson (Patrik.magnusson@ki.se). Researchers interested in using QIMR data can contact Nick Martin (Nick.Martin@qimrberghofer.edu.au) and Sarah Medland (medlandse@gmail.com). For the HRS, phenotypic data are publicly available. Researchers who wish to link genetic data with other HRS measures can apply for access from HRS (http://hrsonline.isr.umich.edu/gwas ). Data from the Atherosclerosis Risk in Communities (ARIC) Study can be accessed through the NHLBI BioLINCC repository (https://biolincc.nhlbi.nih.gov/home/) or by contacting the ARIC Coordinating Center (http://www2.cscc.unc.edu/aric/distribution-agreements). The raw microarray data for the EGCUT cohort are freely available for researchers through managed access control via the Estonian Genome Center's DAC (Scientific Committee of the Estonian Genome Center), as defined by the cohort's consent. All respective requests should be submitted by email to biobank@ut.ee. Data can typically not be released without assessment by a local steering committee with transfer agreements and ethical approval, as the phenotypic data can be sensitive.

I wish to express my gratitude to all participants of these studies, to our data collaborators who are also co-authors on the respective journal articles and to the Complex Traits Genomics Groups around Peter Visscher, who openly shared their technical infrastructure and a know how to use the data. It was incredibly exciting. 


\section{Summary}

To what extent do genes influence when you have your first child and the total number of children that you have? Does the (social) environment change genetic effects on fertility? Do genes lead to spurious associations between life outcomes such as education and age at first birth? Until now, social science research on fertility has largely ignored genetic explanations and instead attributed our fertility behaviour almost exclusively to the social environment; that is: upbringing, postponement of having children in the pursuit of educational attainment and labour force participation, and value change. Yet a growing body of research over the last few decades has demonstrated the relevance of incorporating biological and genetic factors into our understanding of fertility outcomes. Recent advances in biology, molecular genetics, medical sciences, reproductive medicine and evolutionary anthropology have likewise increased the relevance of the interdisciplinary biodemographic approach to fertility. Biodemography is a recent branch of science that integrates biology and demography, focussing on the complementary biological and demographic determinants, and their interactions, to account for the birth and death processes.

This dissertation contains a review article (chapter 1) and four empirical studies (chapters 2, 3, 4 and 5) on the biodemography of fertility. We first summarized what we have learned until now about the biodemography of fertility and outlined future fertile frontiers in this area of research. We then investigated genetic and environmental influences on human fertility, as well as their interplay. We used twin studies as well as large datasets of molecular genetic data - on up to 31,000 individuals from six countries - to quantify the extent to which genes or environmental factors influence human fertility. Research questions answered in the four empirical chapters include the following. How much of the observed differences in women's age at first birth can be explained by genetic differences and how much by environmental differences? Are the genetic bases of age at first birth and number of children ever born the same? Does the historical and geographic environment change the influence of genes on fertility behaviour? Does education 
influence the age at first birth if we control for all family influences - genetic and non-genetic?

How much of the observed differences in the age at first birth of women are associated with genetic differences and how much with environmental differences? In chapter 2, we conducted a study on 4,548 female twins form the United Kingdom born between 1919-68 from the TwinsUK register to investigate the extent to which genetic predispositions are associated age at first birth. We found that genetic dispositions explain 26 percent of the observed differences in the age at first birth, the shared environment of siblings explains 14 percent and the nonshared environment/measurement error 60 percent. Importantly, the exclusion of censored information (i.e. on the childless) changes results non-systematically, and future genetically informed research should aim to integrate this information into their statistical modelling approaches.

The last few years have brought a revolution in the ability to access and analyse molecular genetic data, which for the first time in history now allows us to go beyond indirect inferences about the impact of genes based on twin comparisons to more direct estimates based on single genetic variants distributed across the entire genome in unrelated individuals. These genetic variants are called single nucleotide polymorphisms (SNPs) and allow us to explore the data in new ways by applying new statistical tools.

In chapter 3, we extended our study design by combining data from the TwinsUK register with data on women from the Lifelines Cohort Study in Groningen. Instead of looking at twins, we used genotypic information to construct a genetic relatedness matrix across all 6,758 individuals. This way, we were able to assess the extent to which common genetic variants from the whole genome are associated women's age at first birth and number of children, and how far genetic effects are shared for the two outcomes. We found that around 10 percent of the variance in number of children ever born and 15 percent of the variance in age at first birth are associated with genetic variants, and that genetic effects on both fertility traits overlap.

Are the genetic bases for the age at first birth and number of children ever born the same? In chapter 3 , we applied a recently developed model based on molecular genetic data to estimate the genetic correlation between age at first birth and number 
of children ever born among women from the UK and the Netherlands. We find that genetic effects significantly overlap: around 20 percent of the well-established association between age at first birth and number of children ever born has a genetic basis.

This study also contributes to the debate about whether humans still evolve via natural selection. The question of whether humans are still evolving has been a hotly debated and contested topic. One camp argues that selection pressures on humans have halted due to the decline in mortality in modern societies before and during our reproductive lifespan. The other camp contends that natural selection is still very much occurring in modern populations. They argue that this on the grounds that there is still considerable variation in the number of children people have. In other words, if particular genes are related to higher reproductive success (i.e., having more children), these genes will be passed on with a higher frequency to future generations.

The number of children has been used as a proxy for 'fitness' and additive genetic variance indicates that natural selection occurs within these modern societies: genes that lead to higher reproductive success will have a higher frequency in future generations. In our study, women from the UK and the Netherlands born in the twentieth century who had a genetic predisposition for an earlier age at first birth have had a reproductive advantage across the generations. Genes associated with an earlier age at first birth have been passed on more frequently to the next generation, meaning that natural selection acts not only in historical, but also contemporary populations. However, if genes associated with an earlier age at first birth are more likely to be passed down to the next generation, why is it that younger generations are not having their children at an even earlier age? What we see is that women are doing exactly the opposite in most industrialized nations. Since the 1970s, women are having their first child around 4-5 years later, which is now on average at age 28-29 years. This massive postponement in the age at first birth suggests that socio-environmental influences held as important by social scientists, such as women's educational expansion and entry into the labour market and the widespread use of effective contraception, have had a much stronger influence on fertility trends than natural selection. However, given the fact that both genes and the socio-environment can be shown to matter for fertility, the study also 
emphasizes the need for an integrative research design combining genetics and the social sciences, so as to better understand and predict human fertility.

Does the historical and geographic environment change the influence of genes on fertility behaviour?

Human traits and behaviour are the result of interplay between genetic and environmental factors. Genes provide predispositions for complex traits such as fertility, but environmental conditions determine whether such dispositions will manifest themselves. We know that fertility behavior - such as age at first birth and number of children - varies strongly across historical time and geographical space. To properly understand gene-environment interactions across societies and time, or in other words the context dependency of genetic effects on fertility, we must evaluate the estimated heritability of a trait in different environments.

In chapter 2, we observed that in the UK, genetic influences on age at first birth change across birth cohorts born 1919-68. We found support for the idea that environmental constraints (war or economic crisis) suppress, and normative freedom (sexual revolution) triggers the activation of genetic predispositions that affect fertility.

In chapter 4, we extended this research. Family and twin studies, which suggest that up to 50 percent of individual differences in fertility might be due to genetic differences, implicitly assume that the genes important for fertility are the same across both time and space. Using molecular genetic data (SNPs) from over 30,000 unrelated individuals from six different countries (Australia, Estonia, Netherlands, Sweden, UK, US), we showed that different genes influence fertility in different time periods and different populations, and that the genetic effects consistently related to fertility are small. Across all individuals and environments, common single-nucleotide polymorphisms (SNPs) explained only $\sim 4$ percent of the variance in number of children ever born and age at first birth. We then extended these models to test whether genetic effects are shared across different environments or unique to them. For individuals belonging to the same population and demographic cohort (born before or after the $20^{\text {th }}$ century fertility decline), SNPbased heritability was almost five times higher at 22 percent for number of children, and was 19 percent for age at first birth. Our findings imply that the environment 
strongly modifies genetic effects on the tempo and quantum of fertility, that potentially on-going natural selection is heterogeneous across environments, and that gene-environment interactions may partly account for missing heritability in fertility.

Does education influence the age at first birth if we control for all family influences genetic and non-genetic? Finally, we use the natural experiment of twinning to answer one of the core questions in modern demography. A large body of literature documents a positive relationship between education and age at first birth, on the basis of which it has been argued that education causally influences age at first birth. The supposed causal effect of education on fertility postponement has become a parsimonious and powerful explanation for demographers, not only due to the fact that higher educated women have their first baby at later ages, but also because of the general postponement of childbearing during the second half of the $20^{\text {th }}$ century which accompanied educational expansion. In this study, we challenged the claim that education causally influences the age at first birth, and consequently repudiated the notion that educational expansion is the main cause of recent fertility postponement. We presented within-twin and novel biometric models using a unique dataset of female twins from the UK.

However, this relationship may in part be spurious due to family background factors that cannot be controlled for in most research designs. We investigates to what extent higher education is causally related to later age at first birth timing in a large sample of female twins from the UK $(\mathrm{N}=2,752)$. We presented novel estimates using within-identical twin and biometric models. Our findings show that one year of additional schooling is associated with about half a year later age at first birth in standard models. This fell to only 1.5 months for the within-identical twin model that controls for all shared family background factors (genetic and family environmental). Biometric analyses reveal that it is mostly influences of the family environment - not genetic factors - that cause spurious associations between education and age at first birth. Lastly, we demonstrate using data from the Office for National Statistics that only 1.9 months of the 2.4 years of fertility postponement for birth cohorts 1944-1969 can be attributed to educational expansion based on these estimates. Our results suggest that the prevailing view of a strong causal effect of education has some merit but needs to be qualified. We concluded that the rise in 
educational attainment alone cannot explain differences in fertility timing between cohorts. 
felix-tropf.com

\section{Samenvatting}

In hoeverre beïnvloeden genen de leeftijd waarop $\mathrm{u}$ uw eerste kind krijgt en hoeveel kinderen $\mathrm{u}$ heeft? Heeft de (sociale) omgeving invloed op het tot uiting komen van erfelijke effecten op fertiliteit? Leiden genen tot onechte associaties tussen levensuitkomsten, zoals de relatie tussen opleidingsniveau en leeftijd bij de eerste geboorte? Tot nu toe heeft sociaalwetenschappelijk onderzoek naar voortplanting (of fertiliteit) genetische verklaringen grotendeels genegeerd en in plaats daarvan wordt ons fertiliteitsgedrag bijna uitsluitend toegeschreven aan de sociale omgeving en opvoeding. Een voorbeeld is het uitstellen van het hebben van kinderen vanwege het opleidingsniveau, arbeidsparticipatie en veranderende waarden. Steeds meer onderzoek in de afgelopen decennia laat echter de relevantie zien van het opnemen van biologische en genetische factoren voor ons begrip van fertiliteit. Recente ontwikkelingen in de biologie, moleculaire genetica, medische wetenschappen, voortplantingsgeneeskunde en evolutionaire antropologie hebben de relevantie van een interdisciplinaire benadering om fertiliteit te bestuderen verhoogd. Biodemografie is een recente tak van de wetenschap die de biologie en demografie integreert, gericht op de complementaire biologische en demografische determinanten van en interacties tussen de processen van geboorte en sterfte.

Dit proefschrift bevat een overzichtsartikel (hoofdstuk 1) en vier empirische studies (hoofdstukken 2, 3, 4 en 5) naar de biodemografie van fertiliteit. We vatten eerst samen wat er uit eerder onderzoek is gebleken betreffende de biodemografie van fertiliteit en we schetsen de toekomstige mogelijkheden voor onderzoek op dit gebied. We onderzoeken vervolgens erfelijke en omgevings-invloeden op de fertiliteit, en hun samenspel. We gebruikten zowel tweelingstudies als grote datasets met moleculaire genetische data - resulterend in 31.000 personen uit zes landen - om vast te stellen in hoeverre genen of omgevingsfactoren fertiliteit beïnvloeden. De onderzoeksvragen die zijn beantwoord in de vier empirische hoofdstukken zijn onder andere: Hoeveel van de waargenomen verschillen in de leeftijd bij de eerste geboorte van vrouwen kan worden verklaard door genetische verschillen en hoeveel door verschillen in de omgeving? Zijn de genetische invloeden voor de leeftijd bij de 
eerste geboorte en het aantal kinderen dat ooit geboren wordt hetzelfde? Veranderen verschillen in tijd en plaats, maw in de historische en geografische omgeving, de invloed van genen op fertiliteits gedrag? Verandert de invloed van opleidingsniveau op de leeftijd bij de eerste geboorte, als we controleren voor alle familie-invloeden genetische en niet-genetische?

Hoeveel van de waargenomen verschillen in leeftijd bij de eerste geboorte van vrouwen zijn geassocieerd met genetische verschillen en hoeveel met omgevingsverschillen? In hoofdstuk 2 hebben we een onderzoek uitgevoerd onder 4,548 vrouwelijke tweelingen uit het Verenigd Koninkrijk geboren tussen 1919-1968 (TwinsUK) om te onderzoeken in hoeverre erfelijkheid een rol speelt bij de leeftijd bij de eerste geboorte. We vonden dat genetische factoren $26 \%$ van de waargenomen verschillen in leeftijd bij de eerste geboorte verklaren, de gedeelde omgeving van broers en zussen verklaart $14 \%$ en de niet-gedeelde omgeving en/of meetfout $60 \%$. Belangrijk is dat het uitsluiten van kinderloze individuen de resultaten niet systematisch verandert en toekomstig genetisch geïnformeerd onderzoek moet ernaar streven informatie over kinderloze individuen te integreren in statistische modellen.

De afgelopen jaren hebben geleid tot een revolutie in de mogelijkheid om toegang te krijgen tot en analyseren van moleculair genetische gegevens. Voor het eerst in de geschiedenis is het nu mogelijk om verder te gaan dan het trekken van indirecte conclusies over de invloed van genen door het vergelijken van tweelingen. De invloed van genen kan nu worden geschat door gebruik te maken van gemeten genetische varianten over het hele genoom van ongerelateerde personen.

In hoofdstuk 3 hebben we gegevens uit het TwinsUK register met gegevens over vrouwen uit de Lifelines Cohort Study in Groningen gecombineerd. In plaats van te kijken naar tweelingen, gebruikten we gemeten genotypische informatie om een genetische verwantschap matrix te bouwen van alle 6.758 personen in de data. Zo kunnen we beoordelen in hoeverre gemeenschappelijke genetische varianten (over het gehele genoom) zijn geassocieerd met de leeftijd bij de eerste geboorte en het aantal kinderen van vrouwen en in hoeverre genetische effecten worden gedeeld door beide uitkomsten. We vinden dat ongeveer $10 \%$ van de variantie van het aantal geboren kinderen en $15 \%$ van de variantie in leeftijd bij de eerste geboorte zijn gerelateerd aan genetische varianten en dat de genetische effecten op beide fertiliteitseigenschappen overlappen. 
felix-tropf.com

Zijn de genetische factoren voor de leeftijd bij de eerste geboorte en het aantal geboren kinderen hetzelfde? In hoofdstuk 3, passen we een recent ontwikkeld model gebaseerd op moleculair genetische gegevens toe, om de genetische correlatie tussen leeftijd bij de eerste geboorte en het aantal kinderen van vrouwen uit het Verenigd Koninkrijk en Nederland te schatten. We vinden dat genetische effecten aanzienlijk overlappen en dat ongeveer $20 \%$ van de relatie tussen leeftijd bij de eerste geboorte en het aantal kinderen verklaard kan worden door gedeelde genetische factoren.

Dit onderzoek draagt ook bij aan het debat over de vraag of de mens nog steeds evolueert via natuurlijke selectie. Dit is een veel besproken en omstreden onderwerp. Het ene kamp stelt dat selectieve druk op de mens is gestopt als gevolg van de daling van de sterfte in de moderne samenlevingen voor en tijdens onze reproductieve levensduur. Het andere kamp stelt dat natuurlijke selectie nog steeds erg veel voorkomt in moderne populaties. Zij beweren dat dit wordt toegeschreven aan het feit dat er nog een aanzienlijke variatie is in het aantal kinderen dat mensen hebben. Met andere woorden, als bepaalde genen gerelateerd zijn aan hoger reproductief succes (dwz meer kinderen), zullen deze genen met een hogere frequentie worden doorgegeven aan toekomstige generaties.

Het aantal kinderen is dus gebruikt als een indicator van 'fitness' en additieve genetische variantie geeft aan dat natuurlijke selectie plaatsvindt binnen deze moderne samenlevingen: genen die leiden tot hoger reproductief succes zullen een hogere frequentie in de toekomstige generaties hebben. In onze studie hadden vrouwen uit het Verenigd Koninkrijk en Nederland, geboren in de twintigste eeuw, met een genetische aanleg voor een eerdere leeftijd bij de eerste geboorte een reproductief voordeel. Genen geassocieerd met een jongere leeftijd bij de eerste geboorte werden vaker doorgegeven aan de volgende generatie, wat betekent dat natuurlijke selectie niet alleen in historische populaties werkt, maar ook in moderne populaties. Echter, als genen geassocieerd met een jongere leeftijd bij de eerste geboorte meer kans hebben om te worden doorgegeven aan de volgende generatie, waarom hebben jongere generaties hun kinderen dan niet op een nog jongere leeftijd? Wat we zien is dat vrouwen precies het tegenovergestelde doen in de meeste geïndustrialiseerde landen. Vergeleken met de jaren 1970, hebben vrouwen tegenwoordig hun eerste kind pas 4-5 jaar later - de gemiddelde leeftijd is nu rond 28-29 jaar. Het enorme uitstel in de leeftijd bij de eerste geboorte suggereert dat de 
sociale omgevingsinvloeden, zoals de opleidingsexpansie, de toegang tot de arbeidsmarkt voor vrouwen en het wijdverbreide gebruik van effectieve anticonceptie een veel sterkere invloed heeft op de fertiliteits trends dan natuurlijke selectie. Echter, gezien het feit dat zowel genen als de sociale omgeving belangrijk zijn voor fertiliteit, benadrukt deze studie de noodzaak van een integrale onderzoeksopzet van de genetica en de sociale wetenschappen om de menselijke fertiliteit beter te begrijpen en te voorspellen.

Verandert de historische en geografische omgeving de invloed van genen op fertiliteits gedrag?

Menselijke gedrag is het resultaat van de interactie tussen erfelijke en omgevingsfactoren. Genen bieden aanleg voor complexe eigenschappen, zoals fertiliteit, maar omgevingsfactoren bepalen of dergelijke aanleg tot uiting komt. We weten dat fertiliteitsgedrag - zoals leeftijd bij de eerste geboorte en het aantal kinderen - sterk varieert over de tijd (historisch) en de ruimte (geografisch). Om genomgeving interacties tussen maatschappijen en tijd goed te begrijpen, of om met andere woorden de context-afhankelijkheid van genetische effecten op fertiliteit te bepalen, moeten we de geschatte erfelijkheid van een eigenschap in verschillende omgevingen bekijken.

In hoofdstuk 2 zagen we dat in het Verenigd Koninkrijk de genetische invloeden op de leeftijd bij de eerste geboorte verandert over cohorten geboren tussen 1919 en 1968. We vonden ondersteuning voor het idee dat normatieve vrijheid (seksuele revolutie) leidt tot de activering van genetische aanleg die de fertiliteit beïnvloed terwijl bepaalde omgevingsbeperkingen zoals oorlog of economische crisis deze genetische aanleg juist onderdrukt.

In hoofdstuk 4 hebben we dit onderzoek verder uitgebreid. Familie en tweelingstudies, die suggereren dat tot $50 \%$ van de individuele verschillen in fertiliteit kunnen voortkomen uit genetische verschillen, nemen impliciet aan dat de genen die betrokken zijn bij fertiliteit hetzelfde zijn over tijd en ruimte. Met behulp van moleculair genetische data (SNP's) van meer dan 30.000 niet-verwante individuen uit zes verschillende landen (Australië, Estland, Nederland, Zweden, het Verenigd Koninkrijk, de VS), toonden we aan dat verschillende genen fertiliteit beïnvloeden in verschillende periodes en verschillende populaties en dat de die consistent aan fertiliteit gerelateerde genetische effecten klein zijn. Over alle 
individuen, verklaarden SNPs slechts ongeveer $4 \%$ van de variantie in het aantal geboren kinderen en de leeftijd bij de eerste geboorte. Vervolgens hebben we deze modellen uitgebreid om te testen of genetische effecten worden gedeeld door verschillende omgevingen of dat ze uniek zijn voor specifieke omgevingen. Voor individuen van dezelfde bevolking en uit hetzelfde demografische cohort (geboren voor of na de fertiliteitsdaling van de 20e eeuw) was SNP-gebaseerde erfelijkheid bijna vijf keer hoger; $22 \%$ voor het aantal kinderen en 19\% voor de leeftijd bij de eerste geboorte. Onze resultaten impliceren dat de omgeving de genetische effecten op het tempo en quantum van fertiliteit sterk wijzigt, dat de mogelijke natuurlijke selectie heterogeen is en verschilt per omgevingen, en dat gen-omgeving interacties mede verantwoordelijk kunnen zijn voor de ontbrekende erfelijkheid ('missing heritability') van de fertiliteit.

Verandert de invloed van opleidingsniveau op de leeftijd bij de eerste geboorte, als we controleren voor alle familie invloeden - genetische en niet-genetische? Tot slot maakten we gebruik van tweelingen als natuurlijk experiment om een van de belangrijkste vragen in de moderne demografie beantwoorden. Veel voorgaand onderzoek toont een positieve relatie tussen het opleidingsniveau en de leeftijd bij de eerste geboorte aan en men heeft betoogd dat dit een oorzakelijk verband betreft. Het veronderstelde causale effect van opleidingsniveau op het uitstel van het krijgen van het eerste kind is uitgegroeid tot een simpele en overtuigende verklaring voor demografen, niet alleen voor het feit dat hoger opgeleide vrouwen hun eerste kind op latere leeftijd hebben, maar ook voor het algemene uitstellen van het krijgen van kinderen in de tweede helft van de 20e eeuw als gevolg van de uitbreiding van het hoger onderwijs. In deze studie hebben we de bewering betwist dat het opleidingsniveau de leeftijd bij de eerste geboorte oorzakelijk beïnvloedt en dus dat de onderwijs uitbreiding de belangrijkste reden is voor het recente uitstel van het krijgen van kinderen. We testten dit door middel van de within-twin methode en nieuwe biometrische modellen en met behulp van een unieke dataset van vrouwelijke tweelingen uit het Verenigd Koninkrijk.

De relatie tussen opleidingsniveau en fertiliteit kan gedeeltelijk te wijten zijn aan familie factoren waar niet voor kan worden gecontroleerd in de meeste onderzoeksmethoden. We onderzochten dus in hoeverre het hoger onderwijs oorzakelijk is gerelateerd aan een latere leeftijd bij de eerste geboorte in een grote 
steekproef van vrouwelijke tweelingen uit het Verenigd Koninkrijk $(\mathrm{N}=2,752)$. We presenteren nieuwe schattingen met behulp van de within-identical-twin methode en biometrische modellen. Onze bevindingen tonen aan dat een jaar extra scholing wordt geassocieerd met ongeveer een half jaar latere leeftijd bij de eerste geboorte in de standaard modellen. Dit is teruggebracht tot slechts 1,5 maanden voor de within twin modellen die controleren voor alle gedeelde familie achtergrond factoren (genetische en familie omgeving). Uit de biometrische analyses blijkt dat vooral sociale invloeden van het gezin - niet genetische factoren - de valse associatie tussen het opleidingsniveau en de leeftijd bij de eerste geboorte veroorzaken. Ten slotte tonen we met behulp van gegevens van het Office for National Statistics aan, dat slechts 1,9 maanden van de 2,4 jaar van het uitstel van de eerste geboorte, voor geboortecohorten 1944-1969 kan worden toegeschreven aan opleidingsexpansie op basis van deze schattingen. Deze resultaten suggereren dat de heersende opvatting van een sterk causaal effect van opleidingsniveau genuanceerd moet worden. We concluderen dat alleen (de stijging van) opleidingsniveau niet de verschillen in fertiliteits timing (tussen cohorten) kan verklaren. 


\section{Zusammenfassung}

In wie weit beeinflussen Gene, wann wir unser erstes Kind bekommen oder wie viele Kinder wir in unserem Leben haben? Kann die (soziale) Umwelt den Einfluss von Genen auf Fertilität verändern? Können Gene zu Scheinkorrelationen zwischen Lebensereignissen beitragen, wie beispielsweise zwischen Bildungsabschluss und dem Alter der ersten Geburt? Bisher hat die sozialwissenschaftliche Fertilitätsforschung genetische Erklärungsansätze weitgehend ignoriert und unser Fertilitätsverhalten nahezu ausschließlich durch unsere soziale Umwelt, unsere Erziehung, unser Bildungsniveau, unsere Arbeitsmarktpartizipation sowie unsere Werte und Normen erklärt. Nichtsdestotrotz hat eine wachsende Anzahl von Studien in den vergangenen Jahrzehnten die Relevanz von biologischen und genetischen Einflussfaktoren für unser Verständnis von realisierter Fertilität demonstriert. Die jüngsten Entwicklungen in Biologie, molekularer Genetik, Medizin, Reproduktionsmedizin und evolutionärer Anthropologie haben in ähnlichem Maße die Relevanz des interdisziplinären Ansatzes der Biodemographie in der Fertilitätsforschung genährt. Biodemographie ist ein relativ junger wissenschaftlicher Zweig, der durch die Integration von Biologie und Demographie komplementäre Determinanten aus beiden Feldern sowie deren Zusammenspiel analysiert, um bevölkerungswissenschaftliche Prozesse im Zusammenhang mit Geburt und Tod besser zu verstehen und zu prognostizieren.

Die vorliegende Arbeit beinhaltet einen Überblicksartikel (Kapitel 1) und vier empirische Kapitel (Kapitel 2, 3, 4, 5), die sich mit der Biodemographie, beziehungsweise mit der sozialwissenschaftlichen Genetik von Fertilität beschäftigen. Wir fassen zunächst unser aktuelles Wissen zur Biodemographie von Fertilität zusammen und geben einen Ausblick auf zukünftige Herausforderungen in dieser Forschung. Im Weiteren erforschen wir Gen- und Umwelteinflüsse auf die menschliche Fertilität sowie deren Zusammenspiel. Wir nutzen Zwillingsstudien sowie molekular genetische Datenvon über 31,000 Individuen aus sechs Ländern, um den Einfluss von Genen und (sozialer) Umwelt auf die menschliche Fertilität zu quantifizieren. Forschungsfragen in den empirischen Kapiteln sind unter anderem: 
In wie weit sind Altersunterschiede der Frauen bei ihrer ersten Geburt auf genetische oder (soziale) Umweltfaktoren zurück zu führen? Sind genetische Einflüsse auf das Alter bei der ersten Geburt und auf die Anzahl der Kinder dieselben? Unterscheiden sich genetische Einflüsse auf die menschliche Fertilität nach historischen oder geographischen Kontexten? Hat der Bildungsgrad noch einen Einfluss auf das Alter von Frauen bei ihrer ersten Geburt, wenn wir genetische und nicht-genetische Familieneinflüsseberücksichtigen?

In wie weit sind Altersunterschiede der Frauen bei ihrer ersten Geburt auf genetische oder (soziale) Umweltfaktoren zurück zu führen? In Kapitel 2 studieren wir 4,548 weibliche Zwillinge aus dem TwinsUK Register in Großbritannien, die zwischen 1919 und 1968 geboren wurden, um zu bestimmen, in wie weit genetische Veranlagungen mit dem Alter der ersten Geburt assoziiert sind. Unsere Ergebnisse zeigen, dass 26 Prozent der beobachteten Unterschiede im Alter bei der ersten Geburt auf genetische Veranlagung zurückzuführen sind, 14 Prozent auf die geteilte Umwelt der Zwillinge und 60 Prozent auf die individuelle Umwelt der Zwillinge, respektive Messfehler. Zentral in unserer Studie ist, dass das Nichtberücksichtigen von zensierter Information (genauer: von kinderlosen Frauen, die also kein „Alter bei der ersten Geburt“ haben) die Ergebnisse nicht-systematisch verändert, so dass zukünftige genetisch informierte Forschung die Integration solcher Daten in ihre statistischen Modelle anstreben sollte.

Die vergangenen Jahre haben eine Revolution im Zugang zu und der Analyse von molekular genetischen Daten gebracht, die uns nun zum ersten Mal in der Geschichte erlauben weiter $\mathrm{zu}$ gehen, als mit Vergleichen von Zwillingspaaren indirekte Inferenz über den Einfluss von Genen zu produzieren. Stattdessen können wir mit Hilfe einzelner genetischer Varianten, gemessen über das gesamte Genome hinweg, direkte Schätzungen basiert auf Individuen von verschiedenen Familien abgeben. Diese genetischen Varianten nennt man Einzelnukleotid-Polymorphismen (single nucleotide polymorphisms; SNPs), und sie erlauben uns neue Daten mit neuen statistischen Methoden zu erforschen.

In Kapitel 3 erweitern wir daher unser Studiendesign und kombinieren Daten von TwinsUK mit Daten von der Lifelines Cohort Study in Groningen. Anstellen nur Zwillinge zu studieren, nutzen wir gemessene genetische Informationen um eine genetische-Verwandtschaftsmatrix (genetic-relatedness matrix, GRM) zwischen allen 
felix-tropf.com

6,758 Individuen im Datensatz zu erstellen. Auf diese Weise können wir schätzen, in wie weit gängige genetische Varianten vom gesamten Genom mit dem Alter der ersten Geburt und der Anzahl der Kinder von Frauen assoziiert sind, und zudem, ob die Varianten dieselben sind. Wir finden, dass circa 10 Prozent der Varianz in der Anzahl der Kinder und 15 Prozent der Varianz im Alter der ersten Geburt mit genetischer Varianz assoziiert ist und dass genetische Effekte für beide Fertilitätsvariablen korrelieren.

Sind genetische Einflüsse auf das Alter bei der ersten Geburt und die Anzahl der Kinder dieselben?

In Kapitel 3 verwenden wir aktuell entwickelte statistische Verfahren basiert auf molekular genetischen Daten, um die genetische Korrelation zwischen der Anzahl der Kinder und dem Alter bei der ersten Geburt für Frauen aus Großbritannien und den Niederlanden zu schätzen. Wir finden, dass sich genetische Effekte signifikant überschneiden und circa 20 Prozent der Assoziation zwischen der Anzahl der Kinder und dem Alter bei der ersten Geburt eine genetische Grundlage haben.

Unsere Studie leistet auch einen Beitrag zur aktuellen Diskussion, ob Menschen heutzutage noch durch natürliche Selektion evolvieren. Eine Seite argumentiert, dass Selektionsdruck auf Menschen wegen der schwindenden Sterblichkeit vor und während des reproduktiven Lebensalters heute kein Thema mehr ist. Die andere Seite hält dagegen, dass natürliche Selektion heute sehr wohl noch eine Rolle spielt, da wir weiterhin stets beträchtliche Variation in der Anzahl der Kinder, die Menschen bekommen, sehen. Wenn bestimmte Gene nun mit höherem Reproduktionserfolg (mehr Kindern haben) zusammenhängt, werden diese Gene häufiger an die nächste Generation weitergegeben als andere.

Die Anzahl der Kinder gilt somit als ein Maß für Fitness und additive genetische Varianz in Fitness impliziert, dass natürliche Selektion stattfindet: Gene, die $\mathrm{zu}$ höherem reproduktiven Erfolg führen, haben eine höhere Frequenz in zukünftigen Generationen. In unserer Studie hatten Frauen aus Großbritannien und den Niederlanden, die im 20. Jahrhundert geboren wurden und genetische Veranlagungen für eine frühe erste Geburt hatten einen reproduktiven Vorteil. Gene, die mit einem jüngeren Alter bei der ersten Geburt zusammenhingen, wurden damit häufiger an die folgende Generation weitergegeben als andere, was bedeutet, 
dass natürliche Selektion nicht nur in historischen, sondern auch in zeitgeschichtlichen Gesellschaften vorkommt. Wenn allerdings genetische Veranlagungen für ein jüngeres Alter bei der ersten Geburt immer häufiger werden, weshalb bekommen wir dann heutzutage unser erstes Kind immer später? Frauen bekommen ihr erstes Kind heute im Mittel mit 28-29, das heißt circa 4-5 Jahre später als in den 1970ern. Dieser Trend vom Alter bei der ersten Geburt suggeriert, dass die sozialwissenschaftlichen Einflussfaktoren, wie beispielsweise die Expansionen in Bildung und Arbeitsmarktpartizipation sowie die Einführung der Pille als effektive Verhütungsmethode, einen weitaus stärkeren Einfluss auf Fertilitätstrends hatten als natürliche Selektion. Da die Studie allerdings zeigt, dass sowohl Gene, als auch Umwelteinflüsse für Fertilität relevant sind, betonen wir die Notwendigkeit für integrative Forschungsdesigns an der Schnittstelle von Genetik und Sozialwissenschaften, um menschliches Fertilitätsverhalten besser zu verstehen und vorherzusagen.

Unterscheiden sich genetische Einflüsse auf die menschliche Fertilität zwischen historischen oder geographischen Kontexten?

Menschliche Eigenschaften und Verhaltensweisen resultieren aus dem Zusammenspiel zwischen Genen und Umweltfaktoren. Gene geben eine Veranlagung für komplexe Eigenschaften und Verhalten wie beispielsweise Fertilitätsverhalten, doch die Umweltbedingungen determinieren, ob genetische Dispositionen sich verwirklichen. Wir wissen, dass das Fertilitätsverhalten sowie das Alter bei der ersten Geburt und die Anzahl der Kinder stark zwischen geographischen und historischen Kontexten variieren. Um die Gene-Umwelt Interaktionen über Gesellschaften und Zeit hinweg $\mathrm{zu}$ verstehen, oder in anderen Worten, um die Kontextabhängigkeit genetischer Effekte auf Fertilitätsverhalten zu verstehen, müssen wir genetische Einflüsse auf Fertilitätsverhalten in unterschiedlichen Umwelten betrachten.

In Kapitel 2 sehen wir, dass sich in Großbritannien der Einfluss von Genen auf das Alter bei der ersten Geburt über Geburtskohorten von 1919-1968 hinweg verändert. Unsere Ergebnisse bestätigen die Idee, dass umweltbedingte Einschränkungen wie Krieg oder ökonomische Krisen genetische Einflüsse auf Fertilität verringern und dass normative Freiheit (beispielweise die sexuelle Revolution) diese vergrößert. 
In Kapitel 4 erweitern wir unsere Analyse. Familien- und Zwillingsstudien suggerieren, dass bis zu 50 Prozent der individuellen Unterschiede in Fertilität genetische basiert sein könnten. Sie nehmen damit implizit an, dass Gene, welche einen Einfluss auf Fertilität haben sind, dieselben in unterschiedlichen Kontexten sind. Wir zeigen anhand von molekular genetischen Daten (SNPs) von über 30,000 Individuen aus unterschiedlichen Familien in sechs Ländern (Australien, Estonia, Niederlande, Schweden, Großbritannien und USA), dass sich genetische Einflüsse periodisch und über Bevölkerungen hinweg unterscheiden, und dass genetische Einflüsse kaum konsistent mit Fertilität assoziiert sind. Über alle Individuen und Kontexte hinweg erklären SNPs nur etwa 4 Prozent der Varianz in der Anzahl der Kinder und dem Alter bei der ersten Geburt. Daraufhin erweitern wir die Modelle, um zu testen ob genetische Effekte in unterschiedlichen Kontexten dieseleben sind oder nicht. Für Individuen aus derselben Bevölkerung und demographischen Kohorte (geboren vor oder nach dem Fertilitätsruckgang im 20. Jahrhundert) erklären SNPs zirka fünf Mal zu viel Varianz für die Anzahl der Kinder (22 Prozent) und das Alter bei der ersten Geburt (19 Prozent). Diese Ergebnisse implizieren, dass die (soziale) Umwelt genetische Effekt auf Fertilitätstempo und -quantum stark modifizieren, dass potenzielle, aktuelle natürliche Selektion heterogen in unterschiedlichen Umwelten vorangeht und dass die Gene-Umwelt Interaktion zum Teil verantwortlich ist für die Diskrepanz in der genetischen Varianzaufklärung zwischen Familienstudien und molekular genetischen Studien.

Hat der Bildungsgrad noch einen Einfluss auf das Alter von Frauen bei ihrer ersten Geburt, wenn wir genetische und nicht-genetische Familieneinflüsse berücksichtigen?

Schließlich nutzen wir das natürliche Experiment der Zwillingsstudie, um eine der zentralen Fragen in der modernen Demographie zu beantworten. Eine große Menge an Literatur dokumentiert eine positive Beziehung zwischen Bildung und Alter bei der ersten Geburt und es wurde argumentiert, dass die Bildung ursächlich das Alter bei der ersten Geburt beeinflusse. Der vermeintliche kausale Effekt von Bildung auf das Alter bei der ersten Geburt ist für Bevölkerungswissenschaftler damit eine sparsame und umfassende Erklärung geworden; nicht nur für die Tatsache, dass höher gebildete Frauen älter ihr erstes Kind bekommen, sondern auch für die allgemeine Altersverschiebung bei der ersten Geburt während der zweiten Hälfte des 20. Jahrhunderts aufgrund der 
Bildungsexpansion. In dieser Studie testen wir die Hypothese, dass die Bildung ursächlich das Alter der ersten Geburt beeinflusst und damit, dass die Bildungsexpansion der Hauptgrund für die jüngsten Fertilitätstrends ist. Da diese Assoziation teilweise durch Familieneffekte verzerrt sein kann, die in gewöhnlichen Studien nicht kotrolliert werden können, untersuchen wir den Zusammenhang in einer großen Stichprobe von weiblichen Zwillingen aus Großbritannien $(N=2752)$.

Wir präsentieren neue biometrische Modelle und fixed-effects Modelle für Zwillinge. Unsere Ergebnisse zeigen, dass ein Jahr zusätzliche Schulzeit mit etwa einem halben Jahr höheren Alter bei der ersten Geburt in Standardmodellen verbunden ist. Dies reduziert sich auf nur noch 1.5 Monate in fixed-effects Modellen, die für alle gemeinsamen Familieneinflüsse von Zwillingen (genetische und Familienumwelt) kontrollieren. Biometrische Analysen zeigen, dass vor allem Einflüsse des familiären Umfeldes, nicht die genetischen Faktoren, für nicht-kausale Assoziationen zwischen Bildung und Alter der ersten Geburt verantwortlich sind. Schließlich verwenden wir Daten des Amtes für nationale Statistik und zeigen, dass nur 1.9 Monate der 2.4 Jahre Fertilitätsverzögerung bei den Geburtskohorten von 1944-1969 auf die Bildungsexpansion zurückzuführen sind. Diese Ergebnisse legen nahe, dass die vorherrschende Idee eines starken kausalen Effekts von Bildung auf das Alter der ersten Geburt nuanciert werden muss. Wir schließen daraus, dass die Bildungsexpansion nicht allein die vergangenen Fertilitätstrends erklären kann. 


\section{Résumé}

Dans quelle mesure les gènes influent-ils sur l'âge auquel vous aurez votre premier enfant et le nombre d'enfant que vous aurez? L'environnement social change-t-il l'impact génétique sur la fertilité ? Les gênes provoquent-ils des mises en relation fallacieuses entre des éléments de notre vie tels que l'éducation et l'âge moyen à la naissance du premier enfant? Jusqu'à présent, la recherche en sciences sociales portant sur la fertilité a largement ignoré les explications génétiques et presque exclusivement attribué notre comportement reproductif à l'environnement social et à l'éducation, justifiant ainsi le fait de fonder une famille plus tard au bénéfice du niveau d'instruction, de la participation au marché du travail et en accord avec l'évolution des valeurs morales. Cependant, au cours des dix dernières années, un nombre croissant de chercheurs a démontré l'intérêt d'inclure les facteurs biologiques et génétiques dans notre analyse de la fécondité. Les avancées récentes en biologie, en génétique moléculaire, en médecine, en médecine reproductive et en anthropologie évolutive ont également renforcé la pertinence d'un recours à une approche biodémographique interdisciplinaire dans l'étude de la fertilité. La biodémographie est une discipline scientifique récente qui intègre la biologie à la démographie et se concentre sur les facteurs déterminants complémentaires d'ordre biologique et démographique et leur interaction sur le processus de vie et de mort.

Cette thèse contient un article de revue, qui constitue le premier chapitre, et quatre autres chapitres qui sont des études empiriques sur la biodémographie de la fertilité. Nous résumons en premier lieu ce que l'on a appris jusqu'ici concernant la biodémographie de la fertilité et mettons également en lumière des pistes de recherches fertiles dans ce domaine. Nous examinons ensuite les influences génétiques et environnementales sur la fertilité humaine, ainsi que leurs interactions. Pour cela nous avons eu recours à des études de jumeaux ainsi que de vastes et nombreux ensembles de données de génétique moléculaire, comprenant jusqu'à 31 000 individus à travers six pays différents, afin de quantifier l'ampleur de l'influence des facteurs génétiques ou environnementaux sur la fertilité humaine. Les 
Résumé

problématiques de recherche auxquelles des réponses sont apportées dans les chapitres empiriques sont les suivantes: combien des différences observées dans l'âge moyen à la naissance du premier enfant peuvent-elles être expliquées par des différences d'ordre génétique et combien peuvent l'être par des différences environnementales? Les bases génétiques en rapport avec l'âge moyen à la naissance du premier enfant et celles concernant le nombre d'enfants sont-elles les mêmes ? L'environnement géographique et historique change-t-il l'influence des gènes sur le comportement reproductif? L'éducation influence-t-elle l'âge à la naissance du premier enfant si l'on prend en compte toutes les influences familiales, génétiques et non-génétiques?

Combien des différences observées dans l'âge moyen à la naissance du premier enfant chez les femmes sont associées aux différences génétiques et combien sont associées aux différences environnementales?

Dans notre chapitre 2, nous avons mené une étude sur 4548 jumelles nées au Royaume Uni entre 1919 et 1968 et répertoriées dans le registre TwinsUK afin de chercher à savoir dans quelle mesure les prédispositions génétiques sont associées avec l'âge moyen à la naissance du premier enfant. Nous avons trouvé que les dispositions génétiques expliquent $26 \%$ des différences observées dans l'âge à la naissance du premier enfant. L'environnement partagé par la fratrie explique quant à lui $14 \%$ et les différences d'environnement et la marge d'erreur $60 \%$. Il est important de souligner que l'absence de certaines informations, notamment concernant les personnes sans enfants, altère certains résultats de manière nonsystématique et que la recherche future se basant sur des données génétiques devrait avoir pour ambition d'intégrer ces informations dans ses approches de la modélisation statistique.

Une vraie révolution a eu lieu au cours des dernières années en ce qui concerne les possibilités d'accès à données de génétique moléculaire et leur analyse. Pour la première fois, il est à présent possible d'aller au delà des simples inférences indirectes concernant l'impact des gènes, en comparant des jumeaux à une 
felix-tropf.com

évaluation plus directe grâce à des variantes génétiques individuelles à travers le génome entier d'individus sans lien de parenté. Ces variantes génétiques sont ce que l'on appelle des polymorphismes d'un seul nucléotide (ou SNP pour single nucleotide polymorphism) et nous permettent d'explorer les données de manière nouvelle en appliquant de nouveaux outils statistiques.

Dans le troisième chapitre, nous avons prolongé notre étude en associant des données $\mathrm{du}$ registre TwinsUK à d'autres informations provenant de la base de données de la LifeLines Cohort Study à Groningue. Au lieu de s'intéresser aux jumeaux, nous avons eu recours des informations génotypiques mesurées afin de bâtir une matrice de parenté génétique à travers 6758 individus. Ainsi, nous avons pu évaluer dans quelle mesure des variantes génétiques du génome entier sont associées à l'âge à la naissance du premier enfant et le nombre d'enfants chez les femmes, et dans quelle mesure les effets génétiques sont communs pour les deux résultats. Nous avons trouvé qu'environ $10 \%$ de la variance dans le nombre total d'enfants et $15 \%$ de la variance de l'âge moyen à la naissance du premier enfant sont associés à des variantes génétiques et que les effets génétiques sur ces deux caractéristiques de la fertilité se chevauchent.

Les bases génétiques pour l'âge moyen à la naissance du premier enfant et le nombre moyen d'enfants sont-elles les mêmes?

Dans le troisième chapitre, nous mettons en pratique un modèle récent basé sur des données de génétique moléculaire afin d'estimer la corrélation génétique entre l'âge moyen à la naissance du premier enfant et le nombre total d'enfants chez des femmes originaires du Royaume Uni et des Pays Bas. Nous trouvons que les effets génétiques se chevauchent de manière significative et qu'environ $20 \%$ des associations établies entre l'âge à la naissance du premier enfant et le nombre total d'enfants ont une base génétique.

Cette étude contribue également au débat concernant l'existence uou non d'une évolution continue des humains en fonction d'une sélection naturelle. La 


\section{Résumé}

question de savoir si l'humain continue d'évoluer ou pas est un sujet contesté qui a été abondamment et ardemment débattu. D'une part, il est soutenu que la pression de la sélection sur les humains s'est stoppée en raison de la chute du taux de mortalité dans les sociétés modernes, avant et au cours de notre espérance de vie reproductive. D'autre part, il est affirmé que la sélection naturelle continue d'avoir lieu au sein des populations modernes. Les détracteurs de cette théorie justifient cela par le fait qu'il y a toujours des variations considérables dans le nombre d'enfants auxquels les gens donnent naissance. En d'autres termes, si des gènes spécifiques sont liés à un plus grand succès reproductif (c'est-à-dire au fait d'avoir plus d'enfants), ces gènes seront transmis à des générations futures avec une plus grande fréquence.

Le nombre d'enfants a été utilisé comme un indicateur de valeur adaptative et la variation génétique supplémentaire indique que la sélection naturelle a bien lieu au sein de ces sociétés modernes : les gènes qui conduisent à un plus grand succès reproductif seront plus fréquents au sein des générations futures. Dans notre étude, des femmes originaires du Royaume Uni et des Pays Bas nées au cours du vingtième siècle et qui avaient une prédisposition génétique pour avoir un âge moyen à la naissance du premier enfant plus bas ont eu un avantage reproductif à travers les générations. Les gènes associés avec un âge moyen à la naissance du premier enfant plus bas ont été transmis plus fréquemment aux générations suivantes, ce qui signifie que la sélection naturelle agit non pas seulement sur les populations passées mais aussi sur les populations contemporaines. Cependant, si les gènes associés à un âge moyen à la naissance du premier enfant plus précoce ont plus de chances d'être transmis aux générations suivantes, pourquoi les jeunes générations n'ont-elles pas leurs enfants encore plus tôt ? On constate que femmes mettent l'opposé en pratique dans les nations les plus industrialisées. Depuis les années 1970, les femmes ont leur premier enfant de quatre à cinq ans plus tard que leurs ainées, en moyenne entre 28 et 29 ans actuellement. Ce report important de l'âge moyen à la naissance du premier enfant suggère que les influences socio-environnementales prédites par les sociologues comme étant importantes, telles que l'expansion de éducation des femmes et leur entrée dans le marché du travail, ainsi que l'usage généralisé d'une contraception efficace, ont eu une influence bien plus forte sur les tendances en 
felix-tropf.com

matière de fécondité que la sélection naturelle. Cependant, étant donné qu'il est possible de prouver de manière empirique que les gènes et l'environnement socioéconomique peuvent tous les deux avoir de l'importance en ce qui concerne la fertilité, cette étude insiste aussi sur la nécessité d'une recherche intégrant à la fois la génétique et les sciences sociales afin de mieux comprendre et prédire la fertilité humaine.

L'environnement géographique et historique change-t-il l'influence des gènes sur le comportement reproductif?

Le comportement humain et les caractéristiques humaines sont le résultat de l'interaction entre des facteurs génétiques et environnementaux. Les gènes fournissent des prédispositions concernant des traits telles que la fertilité, mais les conditions environnementales déterminent si ces dispositions vont se manifester. Nous savons que le comportement reproductif, tel que l'âge moyen à la naissance du premier enfant ou le nombre d'enfants, varie fortement à travers le temps historique et l'espace géographique. Afin de bien comprendre les interactions entre les gènes et l'environnement à travers les sociétés et le temps, ou en d'autres termes, le rapport au contexte des effets génétiques sur la fertilité, nous devons donc évaluer l’héritabilité estimée d'un trait dans des environnements différents.

Dans le second chapitre, nous avons observé qu'au Royaume Uni les influences génétiques sur l'âge moyen à la naissance du premier enfant changent à travers les cohortes de naissance nées entre 1919 et 1968. Nous avons trouvé des éléments favorables à l'idée selon laquelle les contraintes environnementales (telles les guerres ou les crises économiques) répriment, alors qu'une liberté formative (comme la révolution sexuelle) déclenche, l'activation des prédispositions génétiques qui affectent la fertilité.

Dans notre chapitre 4, nous avons prolongé cette étude. Les recherches dans le domaine de la famille et les études de jumeaux qui suggèrent que jusqu'à 50\% des différences individuelles en matière de fertilité pourraient être dues à des différences d'ordre génétique, partent implicitement du principe que les gènes qui sont importants pour la fertilité sont les mêmes à travers le temps et l'espace. En utilisant des données de génétique moléculaire (SNP) de plus de 30000 individus sans lien de 
Résumé

parenté et provenant de six pays différents (Australie, Estonie, Etats Unis, Pays Bas, Royaume Uni, Suède), nous avons démontré que des gènes différents influencent la fertilité au cours de différentes périodes et dans différentes populations, et que les effets génétiques systématiquement liés à la fertilité sont faibles. A travers tous les individus et environnements, des polymorphismes de nucléotide unique (SNP) n'ont expliqué qu'environ $4 \%$ de la variance du nombre d'enfants et de l'âge moyen à la naissance du premier enfant. Nous avons ensuite étendu ces modèles afin de tester si les effets génétiques sont partagés à travers des environnements différents ou qui leur sont propres. Dans le cas des individus appartenant à la même population et cohorte démographique (nés avant ou après le déclin de fertilité au vingtième siècle), l'héritabilité basée sur le polymorphisme de nucléotide unique était presque cinq fois supérieure, à $22 \%$ concernant le nombre total d'enfants et à $19 \%$ concernant l'âge moyen à la naissance du premier enfant. Nos résultats suggèrent ainsi que l'environnement modifie fortement les effets génétiques sur les aspects temporels et quantitatifs de la fertilité, qu'une sélection naturelle potentiellement continue est hétérogène à travers les environnements, et que les interactions gènes-environnement pourraient en partie expliquer l'héritabilité manquante en matière de fertilité.

\section{L'éducation influence-t-elle l'âge à la naissance du premier enfant si l'on prend en compte toutes les influences familiales, génétiques et non-génétiques?}

Pour finir, nous utilisons l'expérimentation naturelle du jumelage pour répondre à l'une des questions centrales de la démographie moderne. Une littérature abondante dans ce domaine a démontré un rapport positif entre l'éducation et l'âge à la naissance du premier enfant. L'effet de causalité supposé de l'éducation sur le report de l'âge de reproduction est devenu une explication parcimonieuse et percutante pour les démographes, pas uniquement dans le cas où les femmes ayant accompli une éducation supérieure ont tendance à avoir leur premier enfant plus tard, mais aussi concernant le report général de l'âge de procréation au cours de la seconde moitié du vingtième siècle, qui serait également dû à l'expansion de l'éducation. Dans la présente étude, nous avons mise au défi l'affirmation selon laquelle l'éducation a un effet de causalité sur l'âge à la naissance du premier enfant 
et par conséquent que l'expansion de l'éducation est la raison principale du report actuel de la procréation. Nous avons présenté des modèles basés sur l'étude de jumeaux et des modèles biométriques novateurs en utilisant une base de donnée de jumelles unique provenant du Royaume Uni.

Cependant, ce rapport peut être en partie faussé à cause de facteurs relevant du contexte familial qui ne peuvent être pris en compte dans la plupart des projets de recherche. Nous examinons dans quelle mesure un lien de causalité existe entre l'éducation supérieure et un âge moyen à la naissance du premier enfant plus élevé au sein d'un échantillon large de jumelles provenant du Royaume Uni $(\mathrm{N}=2,752)$. Nous présentons des évaluations nouvelles en utilisant des modèles basés à la fois sur l'étude de jumeaux identiques et des modèles biométriques. Nos résultats montrent qu'une année supplémentaire d'éducation est associée à environ un âge moyen à la naissance du premier enfant plus élevé de six mois en utilisant le modèle standard. Ce résultat ne se trouve réduit que d'un mois et demi par le recours à un modèle basé sur l'étude de jumeaux identiques qui prend ainsi en compte les facteurs communs d'ordre familial (génétiques et de l'ordre de l'environnement familial). Les analyses biométriques révèlent principalement que l'impact de l'environnement familial, et non les facteurs génétiques, cause de fausses associations entre l'éducation et l'âge à la naissance du premier enfant. Enfin, en utilisant des données provenant de l'Office for National Statistics, nous démontrons que seulement 1,9 mois sur les 2,4 années de report de procréation chez les cohortes nées entre 1944 et 1969 pourraient être attribués à l'expansion de l'éducation. Ces résultats suggèrent que l'idée dominante selon laquelle l'éducation engendre un effet de causalité important a du mérite, mais doit nécessite d'être nuancée.

Nous concluons que (l'augmentation dans) la réussite scolaire à elle seule ne peut expliquer les différences de date de procréation (entre les cohortes). 


\section{On the author}

Felix Christian Tropf (born 14.10.1984 in Alzenau, Germany) is a sociologist currently focussing on social science, genetics and fertility. He received his Diploma (Master equivalent) from the University of Bamberg with distinction. For his thesis on sex-differences in elderly care, the German Society for Demography (DGD) honoured him with the Allianz-Newcomer award 2012. From 2011 to 2015, he was appointed as doctoral researcher and lecturer at the ICS Graduate School, University of Groningen. Since September 2015, Felix has been working as a post-doctoral researcher for the Department of Sociology at the University of Oxford and as a Research Fellow at Nuffield College. His research has been published, amongst others, in the Proceedings of the National Academy of Sciences, Demography, JAMA psychiatry, Population Studies and Behavior Genetics. For further information, see felixtropf.com or scan the QR-Code below.

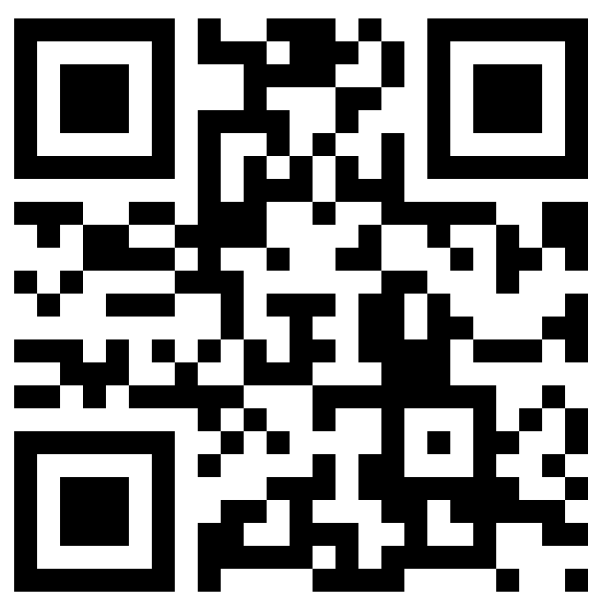


felix-tropf.com

\section{ICS Dissertation Series}

The ICS series presents dissertations of the Interuniversity Center for Social Science Theory and Methodology. Each of these studies aims at integrating explicit theory formation with state of the art empirical research or at the development of advanced methods for empirical research. The ICS was founded in 1986 as a cooperative effort of the universities of Groningen and Utrecht. Since 1992, the ICS expanded to the University of Nijmegen. Most of the projects are financed by the participating universities or by the Netherlands Organization for Scientific Research (NWO). The international composition of the ICS graduate students is mirrored in the increasing international orientation of the projects and thus of the ICS series itself.

1. Cornelis van Liere (1990). Lastige leerlingen. Een empirisch onderzoek naar sociale oorzaken van probleemgedrag op basisscholen. Amsterdam: Thesis Publishers.

2. Marco H.D. van Leeuwen (1990). Bijstand in Amsterdam, ca. 1800-1850. Armenzorg als beheersings- en overlevingsstrategie. ICS-dissertation, Utrecht.

3. Ineke Maas (1990). Deelname aan podiumkunsten via de podia, de media en actieve beoefening. Substitutie of leereffecten? Amsterdam: Thesis Publishers.

4. Marjolein I. Broese van Groenou (1991). Gescheiden netwerken. De relaties met vrienden en verwanten na echtscheiding. Amsterdam: Thesis Publishers.

5. Jan M.M. van den Bos (1991). Dutch EC policy making. A model guided approach to coordination and negotiation. Amsterdam: Thesis Publishers.

6. Karin Sanders (1991). Vrouwelijke pioniers. Vrouwen en mannen met een 'mannelijke' hogere beroepsopleiding aan het begin van hun loopbaan. Amsterdam: Thesis Publishers.

7. Sjerp de Vries (1991). Egoism, altruism, and social justice. Theory and experiments on cooperation in social dilemmas. Amsterdam: Thesis Publishers.

8. Ronald S. Batenburg (1991). Automatisering in bedrijf. Amsterdam: Thesis Publishers.

9. Rudi Wielers (1991). Selectie en allocatie op de arbeidsmarkt. Een uitwerking voor de informele en geïntitutionaliseerde kinderopvang. Amsterdam: Thesis Publishers. 
ICS Dissertation Series

10. Gert P. Westert (1991). Verschillen in ziekenhuisgebruik. ICS-dissertation, Groningen.

11. Hanneke Hermsen (1992). Votes and policy preferences. Equilibria in party systems. Amsterdam: Thesis Publishers.

12. Cora J.M. Maas (1992). Probleemleerlingen in het basisonderwijs. Amsterdam: Thesis Publishers.

13. Ed A.W. Boxman (1992). Contacten en carrière. Een empirisch theoretisch onderzoek naar de relatie tussen sociale netwerken en arbeidsmarktposities. Amsterdam: Thesis Publishers.

14. Conny G.J. Taes (1992). Kijken naar banen. Een onderzoek naar de inschatting van arbeidsmarktkansen bij schoolverlaters uit het middelbaar beroepsonderwijs. Amsterdam: Thesis Publishers.

15. Peter van Roozendaal (1992). Cabinets in multi party democracies. The effect of dominant and central parties on cabinet composition and durability. Amsterdam: Thesis Publishers.

16. Marcel van Dam (1992). Regio zonder regie. Verschillen in en effectiviteit van gemeentelijk arbeidsmarktbeleid. Amsterdam: Thesis Publishers.

17. Tanja van der Lippe (1993). Arbeidsverdeling tussen mannen en vrouwen. Amsterdam: Thesis Publishers.

18. Marc A. Jacobs (1993). Software: Kopen of kopiëren? Een sociaal wetenschappelijk onderzoek onder PC gebruikers. Amsterdam: Thesis Publishers.

19. Peter van der Meer (1993). Verdringing op de Nederlandse arbeidsmarkt. Sector- en sekseverschillen. Amsterdam: Thesis Publishers.

20. Gerbert Kraaykamp (1993). Over lezen gesproken. Een studie naar sociale differentiatie in leesgedrag. Amsterdam: Thesis Publishers.

21. Evelien Zeggelink (1993). Strangers into friends. The evolution of friendship networks using an individual oriented modeling approach. Amsterdam: Thesis Publishers.

22. Jaco Berveling (1994). Het stempel op de besluitvorming. Macht, invloed en besluitvorming op twee Amsterdamse beleidsterreinen. Amsterdam: Thesis Publishers.

23. Wim Bernasco (1994). Coupled careers. The effects of spouse's resources on success at work. Amsterdam: Thesis Publishers. 
felix-tropf.com

24. Liset van Dijk (1994). Choices in child care. The distribution of child care among mothers, fathers and non parental care providers. Amsterdam: Thesis Publishers.

25. Jos de Haan (1994). Research groups in Dutch sociology. Amsterdam: Thesis Publishers.

26. Kwasi Boahene (1995). Innovation adoption as a socio economic process. The case of the Ghanaian cocoa industry. Amsterdam: Thesis Publishers.

27. Paul E.M. Ligthart (1995). Solidarity in economic transactions. An experimental study of framing effects in bargaining and contracting. Amsterdam: Thesis Publishers.

28. Roger Th. A.J. Leenders (1995). Structure and influence. Statistical models for the dynamics of actor attributes, network structure, and their interdependence. Amsterdam: Thesis Publishers.

29. Beate Völker (1995). Should auld acquaintance be forgot...? Institutions of communism, the transition to capitalism and personal networks: the case of East Germany. Amsterdam: Thesis Publishers.

30. Anna M. Cancrinus Matthijsse (1995). Tussen hulpverlening en ondernemerschap. Beroepsuitoefening en taakopvattingen van openbare apothekers in een aantal West Europese landen. Amsterdam: Thesis Publishers.

31. Nardi Steverink (1996). Zo lang mogelijk zelfstandig. Naar een verklaring van verschillen in oriëntatie ten aanzien van opname in een verzorgingstehuis onder fysiek kwetsbare ouderen. Amsterdam: Thesis Publishers.

32. Ellen Lindeman (1996). Participatie in vrijwilligerswerk. Amsterdam: Thesis Publishers.

33. Chris Snijders (1996). Trust and commitments. Amsterdam: Thesis Publishers.

34. Koos Postma (1996). Changing prejudice in Hungary. A study on the collapse of state socialism and its impact on prejudice against gypsies and Jews. Amsterdam: Thesis Publishers.

35. Jooske T. van Busschbach (1996). Uit het oog, uit het hart? Stabiliteit en verandering in persoonlijke relaties. Amsterdam: Thesis Publishers.

36. René Torenvlied (1996). Besluiten in uitvoering. Theorieën over beleidsuitvoering modelmatig getoetst op sociale vernieuwing in drie gemeenten. Amsterdam: Thesis Publishers. 
ICS Dissertation Series

37. Andreas Flache (1996). The double edge of networks. An analysis of the effect of informal networks on cooperation in social dilemmas. Amsterdam: Thesis Publishers.

38. Kees van Veen (1997). Inside an internal labor market: Formal rules, flexibility and career lines in a Dutch manufacturing company. Amsterdam: Thesis Publishers.

39. Lucienne van Eijk (1997). Activity and well being in the elderly. Amsterdam: Thesis Publishers.

40. Róbert Gál (1997). Unreliability. Contract discipline and contract governance under economic transition. Amsterdam: Thesis Publishers.

41. Anne Geerte van de Goor (1997). Effects of regulation on disability duration. ICSdissertation, Utrecht.

42. Boris Blumberg (1997). Das Management von Technologiekooperationen. Partnersuche und Verhandlungen mit dem Partner aus empirisch theoretischer Perspektive. ICS-dissertation, Utrecht.

43. Marijke von Bergh (1997). Loopbanen van oudere werknemers. Amsterdam: Thesis Publishers.

44. Anna Petra Nieboer (1997). Life events and well being: A prospective study on changes in well being of elderly people due to a serious illness event or death of the spouse. Amsterdam: Thesis Publishers.

45. Jacques Niehof (1997). Resources and social reproduction: The effects of cultural and material resources on educational and occupational careers in industrial nations at the end of the twentieth century. ICS-dissertation, Nijmegen.

46. Ariana Need (1997). The kindred vote. Individual and family effects of social class and religion on electoral change in the Netherlands, 1956-1994. ICS-dissertation, Nijmegen.

47. Jim Allen (1997). Sector composition and the effect of education on wages: an international Comparison. Amsterdam: Thesis Publishers.

48. Jack B.F. Hutten (1998). Workload and provision of care in general practice. An empirical study of the relation between workload of Dutch general practitioners and the content and quality of their Care. ICS-dissertation, Utrecht.

49. Per B. Kropp (1998). Berufserfolg im Transformationsprozeß. Eine theoretisch empirische Studie über die Gewinner und Verlierer der Wende in Ostdeutschland. ICS-dissertation, Utrecht. 
felix-tropf.com

50. Maarten H.J. Wolbers (1998). Diploma inflatie en verdringing op de arbeidsmarkt. Een studie naar ontwikkelingen in de opbrengsten van diploma's in Nederland. ICSdissertation, Nijmegen.

51. Wilma Smeenk (1998). Opportunity and marriage. The impact of individual resources and marriage market structure on first marriage timing and partner choice in the Netherlands. ICS-dissertation, Nijmegen.

52. Marinus Spreen (1999). Sampling personal network structures: Statistical inference in ego-graphs. ICS-dissertation, Groningen.

53. Vincent Buskens (1999). Social networks and trust. ICS-dissertation, Utrecht.

54. Susanne Rijken (1999). Educational expansion and status attainment. A crossnational and over-time comparison. ICS-dissertation, Utrecht.

55. Mérove Gijsberts (1999). The legitimation of inequality in state-socialist and market societies, 1987-1996. ICS-dissertation, Utrecht.

56. Gerhard G. Van de Bunt (1999). Friends by choice. An actor-oriented statistical network model for friendship networks through time. ICS-dissertation, Groningen.

57. Robert Thomson (1999). The party mandate: Election pledges and government actions in the Netherlands, 1986-1998. Amsterdam: Thela Thesis.

58. Corine Baarda (1999). Politieke besluiten en boeren beslissingen. Het draagvlak van het mestbeleid tot 2000. ICS-dissertation, Groningen.

59. Rafael Wittek (1999). Interdependence and informal control in organizations. ICSdissertation, Groningen.

60. Diane Payne (1999). Policy making in the European Union: An analysis of the impact of the reform of the structural funds in Ireland. ICS-dissertation, Groningen.

61. René Veenstra (1999). Leerlingen-klassen-scholen. Prestaties en vorderingen van leerlingen in het voortgezet onderwijs. Amsterdam, Thela Thesis.

62. Marjolein Achterkamp (1999). Influence strategies in collective decision making. A comparison of two models. ICS-dissertation, Groningen.

63. Peter Mühlau (2000). The governance of the employment relation. A relational signaling perspective. ICS-dissertation, Groningen.

64. Agnes Akkerman (2000). Verdeelde vakbeweging en stakingen. Concurrentie om leden. ICS-dissertation, Groningen.

65. Sandra van Thiel (2000). Quangocratization: Trends, causes and consequences. ICSdissertation, Utrecht. 
66. Rudi Turksema (2000). Supply of day care. ICS-dissertation, Utrecht.

67. Sylvia E. Korupp (2000). Mothers and the process of social stratification. ICSdissertation, Utrecht.

68. Bernard A. Nijstad (2000). How the group affects the mind: Effects of communication in idea generating groups. ICS-dissertation, Utrecht.

69. Inge F. de Wolf (2000). Opleidingsspecialisatie en arbeidsmarktsucces van sociale wetenschappers. ICS-dissertation, Utrecht.

70. Jan Kratzer (2001). Communication and performance: An empirical study in innovation teams. ICS-dissertation, Groningen.

71. Madelon Kroneman (2001). Healthcare systems and hospital bed use. ICS/ NIVELdissertation, Utrecht.

72. Herman van de Werfhorst (2001). Field of study and social inequality. Four types of educational resources in the process of stratification in the Netherlands. ICSdissertation, Nijmegen.

73. Tamás Bartus (2001). Social capital and earnings inequalities. The role of informal job search in Hungary. ICS-dissertation Groningen.

74. Hester Moerbeek (2001). Friends and foes in the occupational career. The influence of sweet and sour social capital on the labour market. ICS-dissertation, Nijmegen.

75. Marcel van Assen (2001). Essays on actor perspectives in exchange networks and social dilemmas. ICS-dissertation, Groningen.

76. Inge Sieben (2001). Sibling similarities and social stratification. The impact of family background across countries and cohorts. ICS-dissertation, Nijmegen.

77. Alinda van Bruggen (2001). Individual production of social well being. An exploratory study. ICS-dissertation, Groningen.

78. Marcel Coenders (2001). Nationalistic attitudes and ethnic exclusionism in a comparative perspective: An empirical study of attitudes toward the country and ethnic immigrants in 22 countries. ICS-dissertation, Nijmegen.

79. Marcel Lubbers (2001). Exclusionistic electorates. Extreme right wing voting in Western Europe. ICS-dissertation, Nijmegen.

80. Uwe Matzat (2001). Social networks and cooperation in electronic communities. A theoretical-empirical analysis of academic communication and internet discussion groups. ICS-dissertation, Groningen. 
felix-tropf.com

81. Jacques P.G. Janssen (2002). Do opposites attract divorce? Dimensions of mixed marriage and the risk of divorce in the Netherlands. ICS-dissertation, Nijmegen.

82. Miranda Jansen (2002). Waardenoriëntaties en partnerrelaties. Een panelstudie naar wederzijdse invloeden. ICS-dissertation, Utrecht.

83. Anne Rigt Poortman (2002). Socioeconomic causes and consequences of divorce. ICSdissertation, Utrecht.

84. Alexander Gattig (2002). Intertemporal decision making. ICS-dissertation, Groningen.

85. Gerrit Rooks (2002). Contract en conflict: Strategisch Management van Inkooptransacties. ICS-dissertation, Utrecht.

86. Károly Takács (2002). Social networks and intergroup conflict. ICS-dissertation, Groningen.

87. Thomas Gautschi (2002). Trust and exchange, effects of temporal embeddedness and network embeddedness on providing and dividing a surplus. ICS-dissertation, Utrecht.

88. Hilde Bras (2002). Zeeuwse meiden. Dienen in de levensloop van vrouwen, ca. 18501950. Aksant Academic Publishers, Amsterdam.

89. Merijn Rengers (2002). Economic lives of artists. Studies into careers and the labour market in the cultural sector. ICS-dissertation, Utrecht.

90. Annelies Kassenberg (2002). Wat scholieren bindt. Sociale gemeenschap in scholen. ICS-dissertation, Groningen.

91. Marc Verboord (2003). Moet de meester dalen of de leerling klimmen? De invloed van literatuuronderwijs en ouders op het lezen van boeken tussen 1975 en 2000. ICSdissertation, Utrecht.

92. Marcel van Egmond (2003). Rain falls on all of us (but some manage to get more wet than others): Political context and electoral participation. ICS-dissertation, Nijmegen.

93. Justine Horgan (2003). High performance human resource management in Ireland and the Netherlands: Adoption and effectiveness. ICS-dissertation, Groningen.

94. Corine Hoeben (2003). LETS' be a community. Community in Local Exchange Trading Systems. ICS-dissertation, Groningen.

95. Christian Steglich (2003). The framing of decision situations. Automatic goal selection and rational goal pursuit. ICS-dissertation, Groningen. 
96. Johan van Wilsem (2003). Crime and context. The impact of individual, neighborhood, city and country characteristics on victimization. ICS-dissertation, Nijmegen.

97. Christiaan Monden (2003). Education, inequality and health. The impact of partners and life course. ICS-dissertation, Nijmegen.

98. Evelyn Hello (2003). Educational attainment and ethnic attitudes. How to explain their relationship. ICS-dissertation, Nijmegen.

99. Marnix Croes en Peter Tammes (2004). Gif laten wij niet voortbestaan. Een onderzoek naar de overlevingskansen van joden in de Nederlandse gemeenten, 19401945. Aksant Academic Publishers, Amsterdam.

100. Ineke Nagel (2004). Cultuurdeelname in de levensloop. ICS-dissertation, Utrecht.

101. Marieke van der Wal (2004). Competencies to participate in life. Measurement and the impact of school. ICS-dissertation, Groningen.

102. Vivian Meertens (2004). Depressive symptoms in the general population: a multifactorial social approach. ICS-dissertation, Nijmegen.

103. Hanneke Schuurmans (2004). Promoting well-being in frail elderly people. Theory and intervention. ICS-dissertation, Groningen.

104. Javier Arregui (2004). Negotiation in legislative decision-making in the European Union. ICS-dissertation, Groningen.

105. Tamar Fischer (2004). Parental divorce, conflict and resources. The effects on children's behaviour problems, socioeconomic attainment, and transitions in the demographic career. ICS-dissertation, Nijmegen.

106. René Bekkers (2004). Giving and volunteering in the Netherlands: Sociological and psychological perspectives. ICS-dissertation, Utrecht.

107. Renée van der Hulst (2004). Gender differences in workplace authority: An empirical study on social networks. ICS-dissertation, Groningen.

108. Rita Smaniotto (2004). 'You scratch my back and I scratch yours' versus 'Love Thy neighbour'. Two proximate mechanisms of reciprocal altruism. ICS-dissertation, Groningen.

109. Maurice Gesthuizen (2004). The life-course of the low-educated in the Netherlands: Social and economic risks. ICS-dissertation, Nijmegen. 
felix-tropf.com

110. Carlijne Philips (2005). Vakantiegemeenschappen. Kwalitatief en kwantitatief onderzoek naar gelegenheid en refreshergemeenschap tijdens de vakantie. ICSdissertation, Groningen.

111. Esther de Ruijter (2005). Household outsourcing. ICS-dissertation, Utrecht.

112. Frank van Tubergen (2005). The integration of immigrants in cross-national perspective: Origin, destination, and community effects. ICS-dissertation, Utrecht.

113. Ferry Koster (2005). For the time being. Accounting for inconclusive findings concerning the effects of temporary employment relationships on solidary behavior of employees. ICS-dissertation, Groningen.

114. Carolien Klein Haarhuis (2005). Promoting anti-corruption reforms. Evaluating the implementation of a World Bank anti-corruption program in seven African countries (1999-2001). ICS-dissertation, Utrecht.

115. Martin van der Gaag (2005). Measurement of individual social capital. ICSdissertation, Groningen.

116. Johan Hansen (2005). Shaping careers of men and women in organizational contexts. ICS-dissertation, Utrecht.

117. Davide Barrera (2005). Trust in embedded settings. ICS-dissertation, Utrecht.

118. Mattijs Lambooij (2005). Promoting cooperation. Studies into the effects of long-term and short-term rewards on cooperation of employees. ICS-dissertation, Utrecht.

119. Lotte Vermeij (2006). What's cooking? Cultural boundaries among Dutch teenagers of different ethnic origins in the context of school. ICS-dissertation, Utrecht.

120. Mathilde Strating (2006). Facing the challenge of rheumatoid arthritis. A 13-year prospective study among patients and a cross-sectional study among their partners. ICS-dissertation, Groningen.

121. Jannes de Vries (2006). Measurement error in family background variables: The bias in the intergenerational transmission of status, cultural consumption, party preference, and religiosity. ICS-dissertation, Nijmegen.

122. Stefan Thau (2006). Workplace deviance: Four studies on employee motives and selfregulation. ICS-dissertation, Groningen.

123. Mirjam Plantinga (2006). Employee motivation and employee performance in child care. The effects of the introduction of market forces on employees in the Dutch childcare sector. ICS-dissertation, Groningen. 
124. Helga de Valk (2006). Pathways into adulthood. A comparative study on family life transitions among migrant and Dutch Youth. ICS-dissertation, Utrecht.

125. Henrike Elzen (2006). Self-management for chronically ill older people. ICSDissertation, Groningen.

126. Ayse Güveli (2007). New social classes within the service class in the Netherlands and Britain. Adjusting the EGP class schema for the technocrats and the social and cultural specialists. ICS-dissertation, Nijmegen.

127. Willem-Jan Verhoeven (2007). Income attainment in post-communist societies. ICSdissertation, Utrecht.

128. Marieke Voorpostel (2007). Sibling support: The exchange of help among brothers and sisters in the Netherlands. ICS-dissertation, Utrecht.

129. Jacob Dijkstra (2007). The effects of externalities on partner choice and payoffs in exchange networks. ICS-dissertation, Groningen.

130. Patricia van Echtelt (2007). Time-greedy employment relationships: Four studies on the time claims of post-Fordist work. ICS-dissertation, Groningen.

131. Sonja Vogt (2007). Heterogeneity in social dilemmas: The case of social support. ICSdissertation, Utrecht.

132. Michael Schweinberger (2007). Statistical methods for studying the evolution of networks and behavior. ICS-dissertation, Groningen.

133. István Back (2007). Commitment and evolution: Connecting emotion and reason in long-term relationships. ICS-dissertation, Groningen.

134. Ruben van Gaalen (2007). Solidarity and ambivalence in parent-child relationships. ICS-dissertation, Utrecht.

135. Jan Reitsma (2007). Religiosity and solidarity - Dimensions and relationships disentangled and tested. ICS-dissertation, Nijmegen.

136. Jan Kornelis Dijkstra (2007). Status and affection among (pre)adolescents and their relation with antisocial and prosocial behavior. ICS-dissertation, Groningen.

137. Wouter van Gils (2007). Full-time working couples in the Netherlands. Causes and consequences. ICS-dissertation, Nijmegen.

138. Djamila Schans (2007). Ethnic diversity in intergenerational solidarity. ICSdissertation, Utrecht. 
felix-tropf.com

139. Ruud van der Meulen (2007). Brug over woelig water: Lidmaatschap van sportverenigingen, vriendschappen, kennissenkringen en veralgemeend vertrouwen. ICS-dissertation, Nijmegen.

140. Andrea Knecht (2008). Friendship selection and friends' influence. Dynamics of networks and actor attributes in early adolescence. ICS-dissertation, Utrecht.

141. Ingrid Doorten (2008). The division of unpaid work in the household: A stubborn pattern? ICS-dissertation, Utrecht.

142. Stijn Ruiter (2008). Association in context and association as context: Causes and consequences of voluntary association involvement. ICS-dissertation, Nijmegen.

143. Janneke Joly (2008). People on our minds: When humanized contexts activate social norms. ICS-dissertation, Groningen.

144. Margreet Frieling (2008). 'Joint production' als motor voor actief burgerschap in de buurt. ICS-dissertation, Groningen.

145. Ellen Verbakel (2008). The partner as resource or restriction? Labour market careers of husbands and wives and the consequences for inequality between couples. ICSdissertation, Nijmegen.

146. Gijs van Houten (2008). Beleidsuitvoering in gelaagde stelsels. De doorwerking van aanbevelingen van de Stichting van de Arbeid in het CAO-overleg. ICS-dissertation, Utrecht.

147. Eva Jaspers (2008). Intolerance over time. Macro and micro level questions on attitudes towards euthanasia, homosexuality and ethnic minorities. ICS-dissertation, Nijmegen.

148. Gijs Weijters (2008). Youth delinquency in Dutch cities and schools: A multilevel approach. ICS-dissertation, Nijmegen.

149. Jessica Pass (2009). The self in social rejection. ICS-dissertation, Groningen.

150. Gerald Mollenhorst (2009). Networks in contexts. How meeting opportunities affect personal relationships. ICS-dissertation, Utrecht.

151. Tom van der Meer (2009). States of freely associating citizens: Comparative studies into the impact of state institutions on social, civic and political participation. ICSdissertation, Nijmegen.

152. Manuela Vieth (2009). Commitments and reciprocity in trust situations. Experimental studies on obligation, indignation, and self-consistency. ICSdissertation, Utrecht. 
ICS Dissertation Series

153. Rense Corten (2009). Co-evolution of social networks and behavior in social dilemmas: Theoretical and empirical perspectives. ICS-dissertation, Utrecht.

154. Arieke J. Rijken (2009). Happy families, high fertility? Childbearing choices in the context of family and partner relationships. ICS-dissertation, Utrecht.

155. Jochem Tolsma (2009). Ethnic hostility among ethnic majority and minority groups in the Netherlands. An investigation into the impact of social mobility experiences, the local living environment and educational attainment on ethnic hostility. ICSdissertation, Nijmegen.

156. Freek Bucx (2009). Linked lives: Young adults' life course and relations with parents. ICS-dissertation, Utrecht.

157. Philip Wotschack (2009). Household governance and time allocation. Four studies on the combination of work and care. ICS-dissertation, Groningen.

158. Nienke Moor (2009). Explaining worldwide religious diversity. The relationship between subsistence technologies and ideas about the unknown in pre-industrial and (post-) industrial societies. ICS-dissertation, Nijmegen.

159. Lieke ten Brummelhuis (2009). Family matters at work. Depleting and enriching effects of employees' family lives on work outcomes. ICS-dissertation, Utrecht.

160. Renske Keizer (2010). Remaining childless. Causes and consequences from a life Course Perspective. ICS-dissertation, Utrecht.

161. Miranda Sentse (2010). Bridging contexts: The interplay between family, child, and peers in explaining problem behavior in early adolescence. ICS-dissertation, Groningen.

162. Nicole Tieben (2010). Transitions, tracks and transformations. Social inequality in transitions into, through and out of secondary education in the Netherlands for cohorts born between 1914 and 1985. ICS-dissertation, Nijmegen.

163. Birgit Pauksztat (2010). Speaking up in organizations: Four studies on employee voice. ICS-dissertation, Groningen.

164. Richard Zijdeman (2010). Status attainment in the Netherlands, 1811-1941. Spatial and temporal variation before and during industrialization. ICS-dissertation, Utrecht.

165. Rianne Kloosterman (2010). Social background and children's educational careers. The and secondary effects of social background over transitions and over time in the Netherlands. ICS-dissertation, Nijmegen. 
felix-tropf.com

166. Olav Aarts (2010). Religious diversity and religious involvement. A study of religious markets in Western societies at the end of the twentieth century. ICS-dissertation, Nijmegen.

167. Stephanie Wiesmann (2010). 24/7 Negotiation in couples transition to parenthood. ICS-dissertation, Utrecht.

168. Borja Martinovic (2010). Interethnic contacts: A dynamic analysis of interaction between immigrants and natives in Western countries. ICS-dissertation, Utrecht.

169. Anne Roeters (2010). Family life under pressure? Parents' paid work and the quantity and quality of parent-child and family time. ICS-dissertation, Utrecht.

170. Jelle Sijtsema (2010). Adolescent aggressive behavior: Status and stimulation goals in relation to the peer context. ICS-dissertation, Groningen.

171. Kees Keizer (2010). The spreading of disorder. ICS-dissertation, Groningen.

172. Michael Mäs (2010). The diversity puzzle. Explaining clustering and polarization of opinions. ICS-dissertation, Groningen.

173. Marie-Louise Damen (2010). Cultuurdeelname en CKV. Studies naar effecten van kunsteducatie op de cultuurdeelname van leerlingen tijdens en na het voortgezet onderwijs. ICS-dissertation, Utrecht.

174. Marieke van de Rakt (2011). Two generations of crime: The intergenerational transmission of convictions over the life course. ICS-dissertation, Nijmegen.

175. Willem Huijnk (2011). Family life and ethnic attitudes. The role of the family for attitudes towards intermarriage and acculturation among minority and majority groups. ICS-dissertation, Utrecht.

176. Tim Huijts (2011). Social ties and health in Europe. Individual associations, crossnational variations, and contextual explanations. ICS-dissertation, Nijmegen.

177. Wouter Steenbeek (2011). Social and physical Disorder. How community, business presence and entrepreneurs influence disorder in Dutch neighborhoods. ICSdissertation, Utrecht.

178. Miranda Vervoort (2011). Living together apart? Ethnic concentration in the neighborhood and ethnic minorities' social contacts and language practices. ICSdissertation, Utrecht.

179. Agnieszka Kanas (2011). The economic performance of immigrants. The role of human and social capital. ICS-dissertation, Utrecht. 
180. Lea Ellwardt (2011). Gossip in organizations. A social network study. ICSdissertation, Groningen.

181. Annemarije Oosterwaal (2011). The gap between decision and implementation. Decision making, delegation and compliance in governmental and organizational settings. ICS-dissertation, Utrecht.

182. Natascha Notten (2011). Parents and the media. Causes and consequences of parental media socialization. ICS-dissertation, Nijmegen.

183. Tobias Stark (2011). Integration in schools. A process perspective on students' interethnic attitudes and interpersonal relationships. ICS-dissertation, Groningen.

184. Giedo Jansen (2011). Social cleavages and political choices. Large-scale comparisons of social class, religion and voting behavior in Western democracies. ICS-dissertation, Nijmegen.

185. Ruud van der Horst (2011). Network effects on treatment results in a closed forensic psychiatric setting. ICS-dissertation, Groningen.

186. Mark Levels (2011). Abortion laws in European countries between 1960 and 2010. Legislative developments and their consequences for women's reproductive decisionmaking. ICS-dissertation, Nijmegen.

187. Marieke van Londen (2012). Exclusion of ethnic minorities in the Netherlands. The effects of individual and situational characteristics on opposition to ethnic policy and ethnically mixed neighbourhoods. ICS-dissertation, Nijmegen.

188. Sigrid M. Mohnen (2012). Neighborhood context and health: How neighborhood social capital affects individual health. ICS-dissertation, Utrecht.

189. Asya Zhelyazkova (2012). Compliance under controversy: analysis of the transposition of European directives and their provisions. ICS-dissertation, Utrecht.

190. Valeska Korff (2012). Between cause and control: Management in a humanitarian organization. ICS-dissertation, Groningen.

191. Maike Gieling (2012). Dealing with diversity: Adolescents' support for civil liberties and immigrant rights. ICS-dissertation, Utrecht.

192. Katya Ivanova (2012). From parents to partners: The impact of family on romantic relationships in adolescence and emerging adulthood. ICS-dissertation, Groningen.

193. Jelmer Schalk (2012). The performance of public corporate actors: Essays on effects of institutional and network embeddedness in supranational, national, and local collaborative contexts. ICS-dissertation, Utrecht. 
felix-tropf.com

194. Alona Labun (2012). Social networks and informal power in organizations. ICSdissertation, Groningen.

195. Michal Bojanowski (2012). Essays on social network formation in heterogeneous populations: Models, methods, and empirical analyses. ICS-dissertation, Utrecht.

196. Anca Minescu (2012). Relative group position and intergroup attitudes in Russia. ICS-dissertation, Utrecht.

197. Marieke van Schellen (2012). Marriage and crime over the life course. The criminal careers of convicts and their spouses. ICS-dissertation, Utrecht.

198. Mieke Maliepaard (2012). Religious trends and social integration: Muslim minorities in the Netherlands. ICS-dissertation, Utrecht.

199. Fransje Smits (2012). Turks and Moroccans in the Low Countries around the year 2000: Determinants of religiosity, trend in religiosity and determinants of the trend. ICS-dissertation, Nijmegen.

200. Roderick Sluiter (2012). The diffusion of morality policies among Western European countries between 1960 and 2010. A comparison of temporal and spatial diffusion patterns of six morality and eleven non-morality policies. ICS-dissertation, Nijmegen.

201. Nicoletta Balbo (2012). Family, friends and fertility. ICS-dissertation, Groningen.

202. Anke Munniksma (2013). Crossing ethnic boundaries: Parental resistance to and consequences of adolescents' cross-ethnic peer relations. ICS-dissertation, Groningen.

203. Anja Abendroth (2013). Working women in Europe. How the country, workplace, and family context matter. ICS-dissertation, Utrecht.

204. Katia Begall (2013). Occupational hazard? The relationship between working conditions and fertility. ICS-dissertation, Groningen.

205. Hidde Bekhuis (2013). The popularity of domestic cultural products: Cross-national differences and the relation to globalization. ICS-dissertation, Utrecht.

206. Lieselotte Blommaert (2013). Are Joris and Renske more employable than Rashid and Samira? A study on the prevalence and sources of ethnic discrimination in recruitment in the Netherlands using experimental and survey data. ICS-dissertation, Utrecht.

207. Wiebke Schulz (2013). Careers of men and women in the 19th and 20th centuries. ICS-dissertation, Utrecht.

208. Ozan Aksoy (2013). Essays on social preferences and beliefs in non-embedded social dilemmas. ICS-dissertation, Utrecht. 
209. Dominik Morbitzer (2013). Limited farsightedness in network formation. ICSdissertation, Utrecht.

210. Thomas de Vroome (2013). Earning your place: The relation between immigrants' economic and psychological integration in the Netherlands. ICS-dissertation, Utrecht.

211. Marloes de Lange (2013). Causes and consequences of employment flexibility among young people. Recent developments in the Netherlands and Europe. ICS-dissertation, Nijmegen.

212. Roza Meuleman (2014). Consuming the nation. Domestic cultural consumption: its stratification and relation with nationalist attitudes. ICS-dissertation, Utrecht.

213. Esther Havekes (2014). Putting interethnic attitudes in context. The relationship between neighbourhood characteristics, interethnic attitudes and residential behaviour. ICS-dissertation, Utrecht.

214. Zoltán Lippényi (2014). Transitions toward an open society? Intergenerational occupational mobility in Hungary in the 19th and 20th centuries. ICS-dissertation, Utrecht.

215. Anouk Smeekes (2014). The presence of the past: Historical rooting of national identity and current group dynamics. ICS-dissertation, Utrecht.

216. Michael Savelkoul (2014). Ethnic diversity and social capital. Testing underlying explanations derived from conflict and contact theories in Europe and the United States. ICS-dissertation, Nijmegen.

217. Martijn Hogerbrugge (2014). Misfortune and family: How negative events, family ties, and lives are linked. ICS-dissertation, Utrecht.

218. Gina Potarca (2014). Modern love. Comparative insights in online dating preferences and assortative mating. ICS-dissertation, Groningen.

219. Mariska van der Horst (2014). Gender, aspirations, and achievements: Relating work and family aspirations to occupational outcomes. ICS-dissertation, Utrecht.

220. Gijs Huitsing (2014). A social network perspective on bullying. ICS dissertation, Groningen.

221. Thomas Kowalewski (2015). Personal growth in organizational contexts. ICSdissertation, Groningen.

222. Manu Muñoz-Herrera (2015). The impact of individual differences on network relations: Social exclusion and inequality in productive exchange and coordination games. ICS-dissertation, Groningen. 
felix-tropf.com

223. Tim Immerzeel (2015). Voting for a change. The democratic lure of populist radical right parties in voting behavior. ICS-dissertation, Utrecht.

224. Fernando Nieto Morales (2015). The control imperative: Studies on reorganization in the public and private sectors. ICS-dissertation, Groningen.

225. Jellie Sierksma (2015). Bounded helping: How morality and intergroup relations shape children's reasoning about helping. ICS-dissertation, Utrecht.

226. Tinka Veldhuis (2015). Captivated by fear. An evaluation of terrorism detention policy. ICS-dissertation, Groningen.

227. Miranda Visser (2015). Loyality in humanity. Turnover among expatriate humanitarian aid workers. ICS-dissertation, Groningen.

228. Sarah Westphal (2015). Are the kids alright? Essays on postdivorce residence arrangements and children's well-being. ICS-dissertation, Utrecht.

229. Britta Rüschoff (2015). Peers in careers: Peer relationships in the transition from school to work. ICS-dissertation, Groningen.

230. Nynke van Miltenburg. (2015). Cooperation under peer sanctioning institutions: Collective decisions, noise, and endogenous implementation. ICS-dissertation, Utrecht.

231. Antonie Knigge (2015). Sources of sibling similarity. Status attainment in the Netherlands during modernization. ICS-dissertation, Utrecht.

232. Sanne Smith (2015). Ethnic segregation in friendship networks. Studies of its determinants in English, German, Dutch, and Swedish school classes. ICSdissertation, Utrecht.

233. Patrick Präg (2015). Social stratification and health. Four essays on social determinants of health and wellbeing. ICS-dissertation, Groningen.

234. Wike Been (2015). European top managers' support for work-life arrangements. ICSdissertation, Utrecht.

235. André Grow (2016). Status differentiation: New insights from agent-based modeling and social network analysis. ICS-dissertation, Groningen.

236 Jesper Rözer (2016). Family and personal networks. How a partner and children affect social relationships. ICS-dissertation, Utrecht.

237 Kim Pattiselanno (2016). At your own risk: The importance of group dynamics and peer processes in adolescent peer groups for adolescents' involvement in risk behaviors. ICS- dissertation, Groningen. 
ICS Dissertation Series

238 Vincenz Frey (2016). Network formation and trust. ICS-dissertation, Utrecht.

239 Rozemarijn van der Ploeg (2016). Be a buddy, not a bully? Four studies on social and emotional processes related to bullying, defending, and victimization. ICSdissertation, Groningen.

240 Tali Spiegel (2016). Identity, career trajectories and wellbeing: A closer look at individuals with degenerative eye conditions. ICS- dissertation, Groningen.

241 Felix Tropf (2016). Social Science Genetics and Fertility. ICS-dissertation, Groningen. 